\title{
An Integrated Framework to Quantify the Impact of Competency factors on Project Performance
}

\author{
By
}

Mina N. Naguib

A thesis submitted in partial fulfillment of the requirements for the degree of

\author{
Master of Science
}

(Civil and Environmental Engineering)

\author{
at the \\ UNIVERSITY OF WISCONSIN - MADISON
}

2020 


\section{Acknowledgments}

I would like to begin my dissertation by thanking God, without whom nothing is possible.

I would like to thank everyone who supported me throughout this research. Specifically, I would like to express my deepest gratitude to my father, Nader Z. Naguib, and my mother, Aml K. Zakhary, whose support and love have always been overwhelming. I would like also to extend my thankfulness to my father, who sacrificed a lot, especially when he moved from California to Madison to stay with me during my master's journey. I would like to express my deepest grief for losing my grandmother, three months before my graduation, I wish you could be there to celebrate together this moment. I know that you are in a better place now and I hope you always remember me in your prayers.

I would like to express my gratitude and appreciation to my academic advisor and the chair of the Construction Engineering and Management program Dr. Awad S. Hanna for his guidance, patience, and support throughout my graduate studies at the University of Wisconsin-Madison.

I would also like to share my appreciation to my dissertation committee for their valuable advice and guidance: Prof. Jeffrey Russell, Vice Provost for Lifelong Learning, and Dean, Division of Continuing Studies; and Prof. Zhenhua Zhu, Assistant Professor of Civil and Environmental Engineering.

I am also grateful to Dr. Wafik Lotfallah and Dr. Wei-Yin Loh for their imperative guidance, thorough feedback and persistent efforts in advancing both my mathematical and statistical knowledge. Your inputs have been influential to this research and my growth.

I would also like to thank Mr. Gary Whited, the manager of the Construction and Materials Support Center, for his valuable advice whenever I needed it. I worked with him in assessing the quality management program for the Wisconsin Department of Transportation. 
I thank all my fellow graduate students who have taken their time to discuss and enrich my work. Each of their intelligence and dedication has challenged me to grow. Especially, I would like to thank my colleague and friend Ebrahim S. Eldamnhoury. We have shared this experience from our undergraduate studies at the American University in Cairo and here we now graduating with master's degrees from UW-Madison. Thank you for pushing and motivating me through the hard times in these past two years. I would also like to acknowledge the insightful guidance of my former colleague, now an Assistant professor at the University of Kentucky, Dr. Hala Nassereddine, and for being a mentor and cooperative in giving her support. I would like also to thank my companion Hiba Jalloul for the good memories that we made together, the long hours that we spent studying together at College Library, and the positive energy that she always bestowed to me during difficult times. I would like to thank the current graduate students Joe Said and Eyad Reda for their encouragement during my graduate career.

Finally, thanks go to Amy Gaeta for her careful attention to language and clarity during the editing process.

It is my hope that this dissertation exhibits my gratefulness to all those involved.

Mina Naguib

April 28 ${ }^{\text {th }}, 2020$ 


\begin{abstract}
Construction companies measure their performance by how well a construction project delivers relative to their goals. The performance of construction projects is governed by merging many events and interactions with different participants and processes in a constantly changing environment. Many such occurrences and interactions are defined as "competencies". These competencies are the inputs that can be quantified and used to distinguish between the poorestperforming and best-performing projects.

Evaluation of project performance has gained significant attention in the construction domain. Within this conversation, one difficulty is that project management literature review does not provide a coherent nor commonly accepted definition of project success. A project's success could be defined differently depending on the stakeholders, since every individual has a subjective set of success requirements. Thus, determining whether a project is a success is a complex problem and a project cannot always be seen as a total successful or complete failure. In the current construction industry, much effort has been spent on precisely measuring and controlling individual performance metrics (i.e. cost) and determining performance indices for them. However, there have been far fewer comprehensive indices that evaluate project performance across numerous performance metrics such as cost, schedule, safety, quality, and communication. Many previous studies have used these performance metrics as ex-post lagging measures to assess the performance of completed projects. Yet, these metrics do not leverage any opportunity to improve the success probability early in the project and serve only as a historic review. What is crucial, but often missed, is that the performance of a construction project is driven by diverse and numerous leading factors that occur among and across project phases. These factors are defined in this research as "competency factors."
\end{abstract}


Project performance and competency factors are two distinct terms, thus not interchangeable. Project competencies are leading factors to project success, hence forecasting tools enabling project managers to predict successful projects. Identifying competency factors is equivalent to asking, “what does it take to be successful in the project?” while investigating performance measures asks, “how will we know whether the project has been successful or not?” In other words, competency factors are leading indicators that focus on achieving success, while project performance focuses on identifying an actual project's success status. Identifying project competencies early in the project can help contractors to better manage construction projects by understanding what causes projects to achieve its goals and outcomes

Previous research also focused on either a limited set of project competencies, a single phase of construction, or both without combining the project competencies into an integrated framework to measure project success. Most of these studies undermined how the competency factors selection process attempts to capture the main events and interactions that occur throughout the main project phases. Additionally, most of the studies either lacked a quantitative assessment of project competencies or overlooked the unequal contributions of different competencies to project performance.

Previous research has considered, in many situations, how project competencies are a measure of project performance. But. they did not investigate project competencies as a prerequisite for project performance, or the fact that project competencies are leading indicators of project performance improvement. In addition, none of the previous studies evaluated the relative impact of the different project competencies on project performance. Therefore, to overcome these limitations of previous research, this thesis develops a comprehensive framework 
and methodology for evaluating project competencies and identifying their relationship to project performance.

The scope of this research is to comprehensively investigate project competencies as leading indicators of project performance by using two robust, data-driven weighting mathematical frameworks. The objectives are to 1) create a comprehensive performance score named the Project Performance Assessment (PPA) score by combining eight performance metrics spanning over six performance areas: cost, schedule, productivity, safety, quality, and communication; 2) benchmark successful projects from less-than-successful projects by performing K-means clustering; 3) compute a customized competency category-specific score from various competency factors spanning nine categories: alignment \& team integration, planning \& design, procurement management, risk management, health, safety \& environmental, quality management, labor productivity, execution \& commissioning, and change management \& project control; 4) assess the overall effect of each increased competency category-specific score on the likelihood of a project being a successful or less-than successful project; 5) quantitatively assess the relative contribution of each competency category to the PPA score and provide one unique comprehensive score named the Competency Category Assessment (CCA) Score; 6) utilize a developed regression model to examine the degree of association between the CCA score and the PPA score, which construction practitioners can use to predict the overall performance score of a project. The final stage is to assess the models' robustness and validation to determine their reliability and reasonability, as well as testing the models’ future predictability. This study will allow construction practitioners to comprehensively assess both their project's competencies and performance using precise mathematics. Thus, the results of this study can assist the construction industry in accurately identifying leverage training and development areas for improvement. 
The results of this analysis showed that the top three metrics are: construction schedule growth, construction cost growth, and \% delta having 18.43\%, 17.38\%, and 15.22\%, respectively. On the other hand, the percentage of rework and the number of lost-time injuries per 1 million dollars have the lowest contribution in calculating the PPA score. The analysis also concluded that successful projects outperform less-than-successful projects, as they have $16 \%$ average cost savings, $42 \%$ average schedule reduction, $10 \%$ average productivity improvement, $7 \%$ average less rework and 66\% average increase in communication efficiency.

The top-weighted competency factors in each category were found to be: 1) project team has the expertise to take on the project; 2) IFC drawings are issued to support the construction activity; 3) all bulky materials needed for the start of construction are purchased \& onsite; 4) risks impacting the construction phase of the project are well assessed and the risk monitoring and response framework are well defined in terms of cost; 5) quality control plan is communicated among the project team; 6) a plan to secure and retain skilled labor and a craft evaluation/qualification program are in place; 7) a coordination plan is well defined between all project parties; 8) change management roles and responsibilities are well defined to project parties. After computing the competency-category specific scores for the eight categories, it was found that successful projects outperform less-than-successful projects by an average of $1.3 \times-1.9 \times$ in all eight categories. This shows that being competent in all categories will increase the likelihood of a project being successful.

The study also highlighted the importance of pre-construction planning \& design and change management \& project control towards project success, as they are the highest weighted categories, $18.61 \%$, and $16.37 \%$, respectively. Therefore, the construction industry should focus more on preconstruction activities because they are a critical link to all future operations of a 
project. Moreover, the median/mean for CCA score for successful projects $(83.2 / 80.4 \%)$ is found to be higher than less-than-successful projects (48.1/44.4\%). Last, but not least, regression analysis is performed to examine the degree of association between the CCA score and PPA score; given that the assumptions of regression analysis are met, the derived model has a coefficient of multiple determination $\left(R^{2}\right)$ of $51.1 \%$ and correlation accuracy of $71.5 \%$. Model validation is conducted using five-fold cross validation and the testing of 20 new projects introduced to the model to check the reliability and reasonability of the developed model. 


\section{Table of Contents}

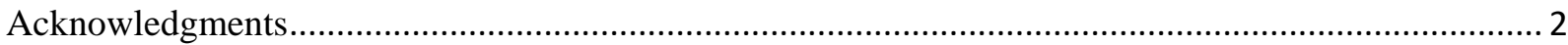

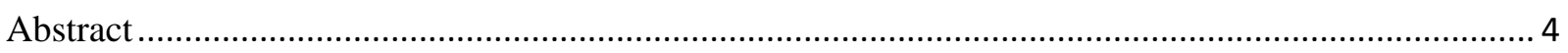

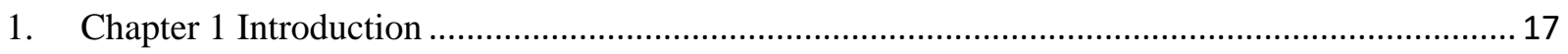

1.1. Delivering Value in a Demanding Environment..................................................................... 18

1.2. Business Imperatives: Improving Project Management .................................................... 22

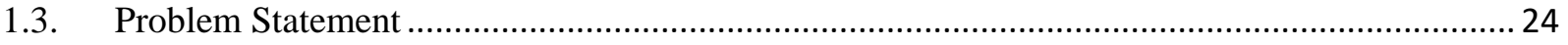

1.4. Objective Statements and Research Contribution................................................................ 26

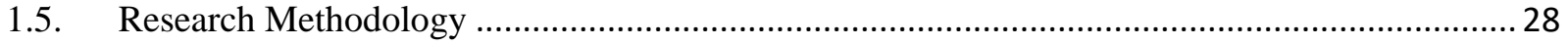

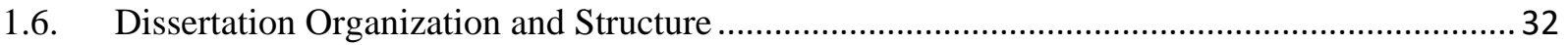

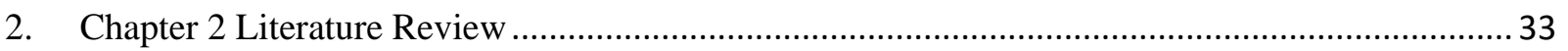

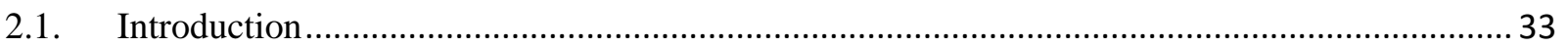

2.2. Review of Project Competencies and Project Performance Frameworks ..................................33

2.2.1. Project Success and Project Performance ...................................................................... 34

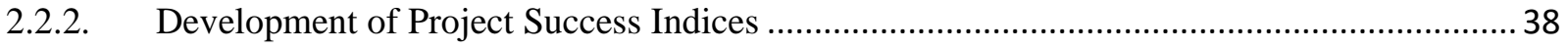

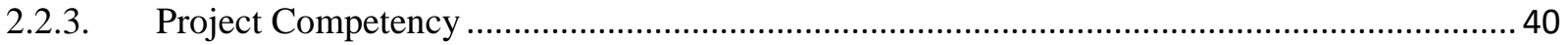

2.2.3.1. Alignment Process \& Team Integration........................................................................... 41

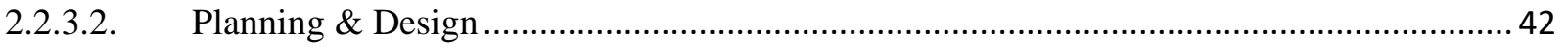

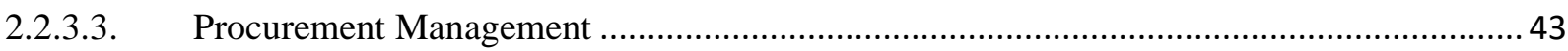

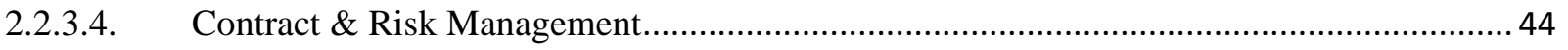

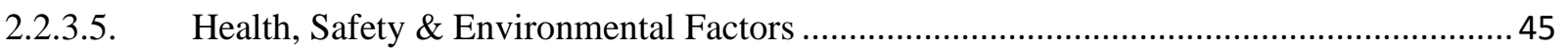

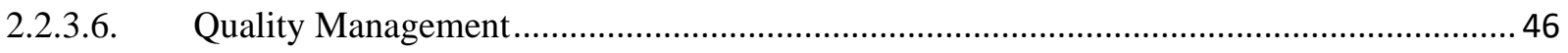

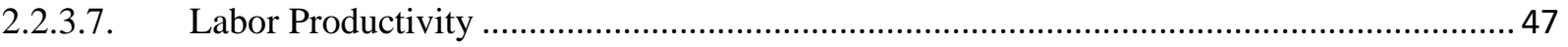

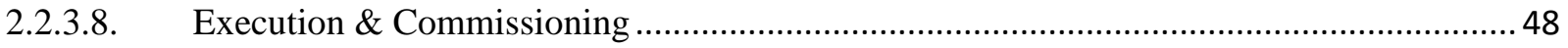

2.2.3.9. Change Management \& Project Control ........................................................................ 49

2.2.4. Project Competencies and Their Relationship to Project Performance ...............................50

2.3. Summary Table of Previous Research Studies ...................................................................... 58

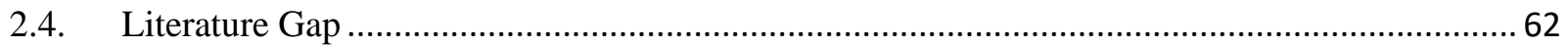

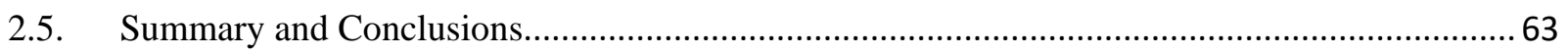

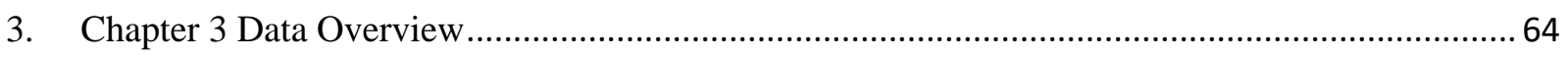

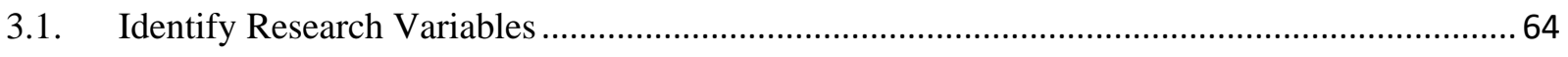

3.1.1. Performance Metrics Assessment Selection Criteria .......................................................... 64

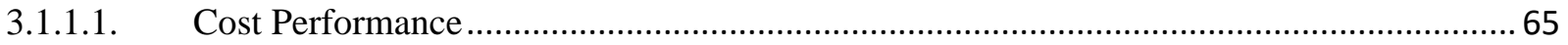




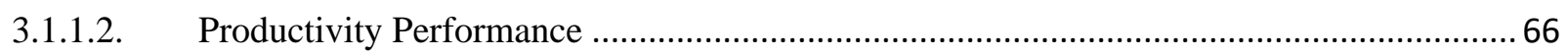

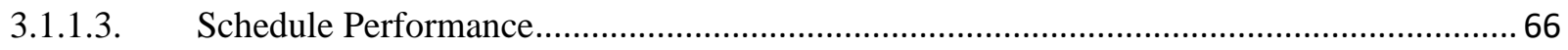

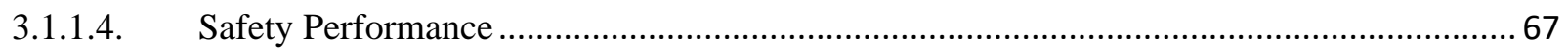

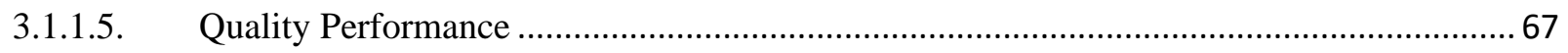

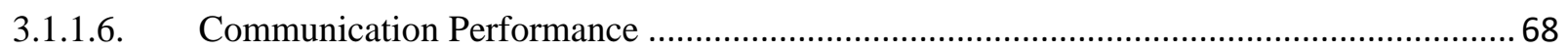

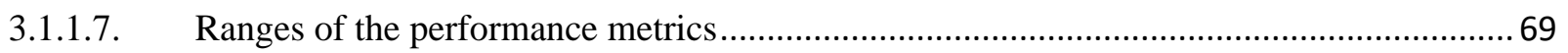

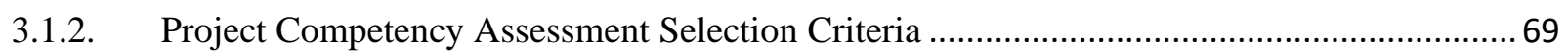

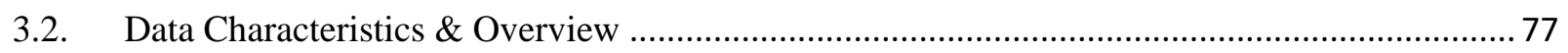

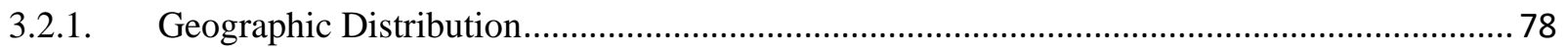

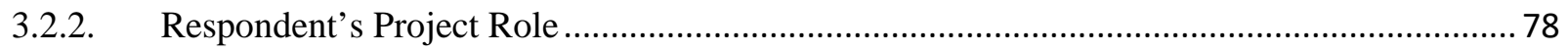

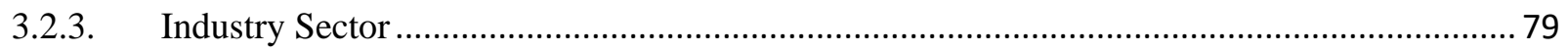

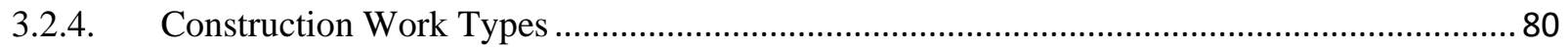

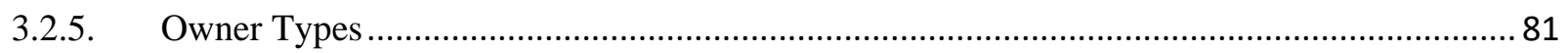

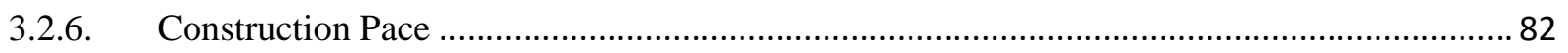

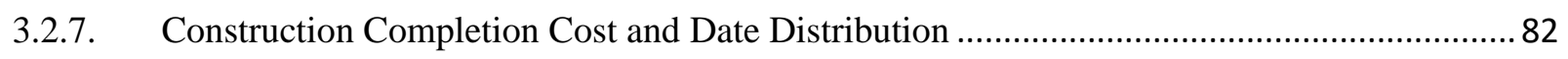

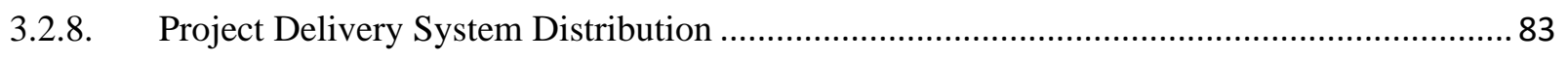

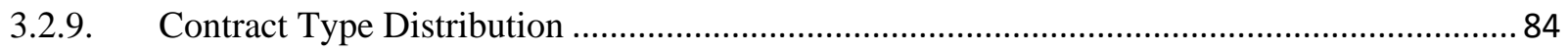

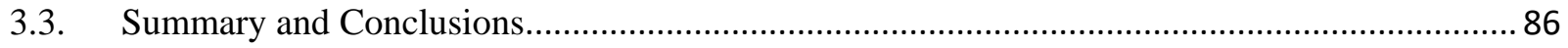

4. Chapter 4 Quantitative Model of Project Performance................................................................ 87

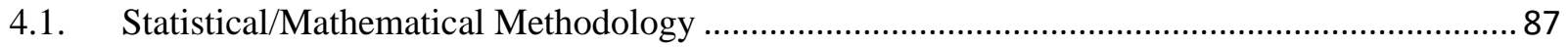

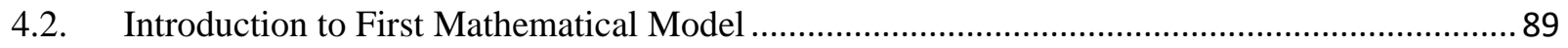

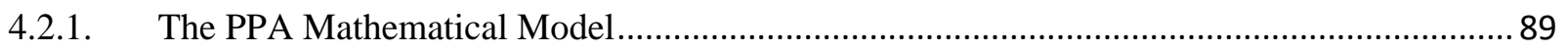

4.2.2. Project Performance Assessment (PPA) Results .......................................................... 91

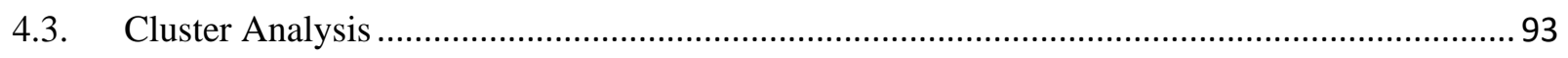

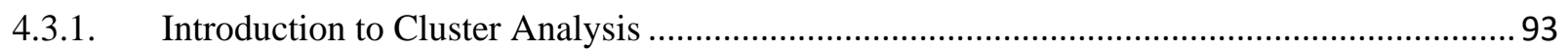

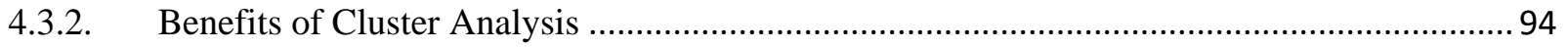

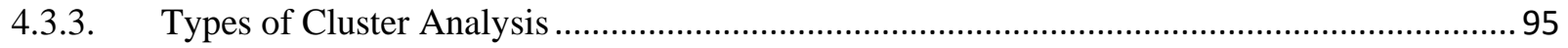

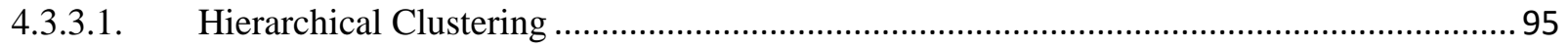

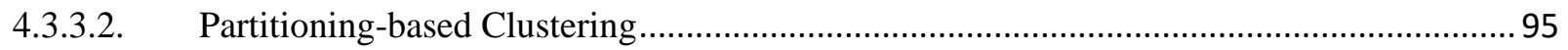

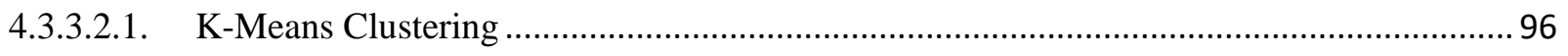

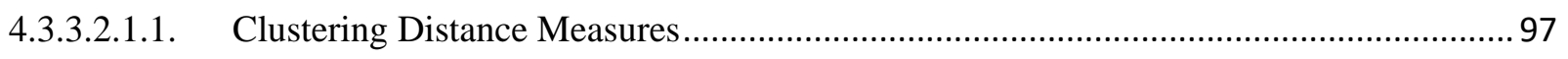

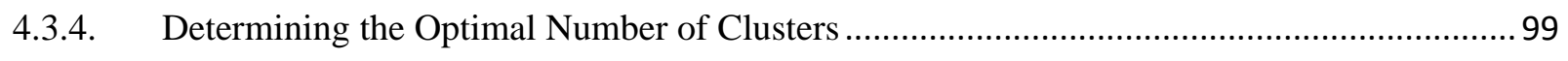

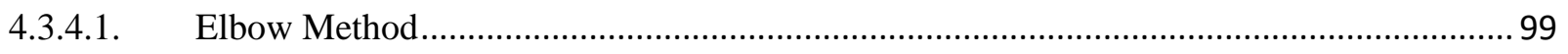




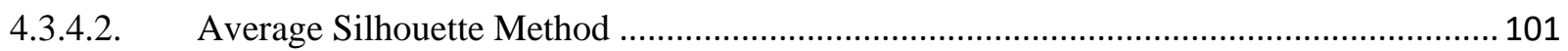

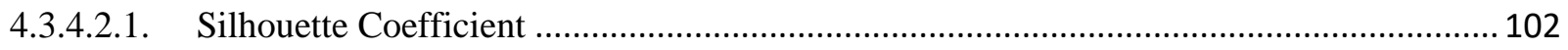

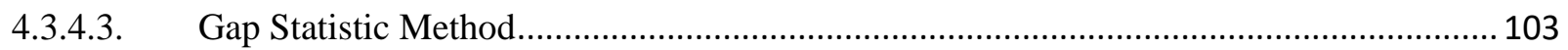

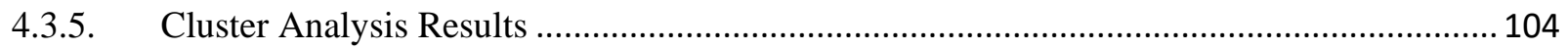

4.4. Project Performance Assessment (PPA) Score Analysis ..................................................... 106

4.5. Performance Metrics vs. PPA Scores/Project Status Assessment........................................... 107

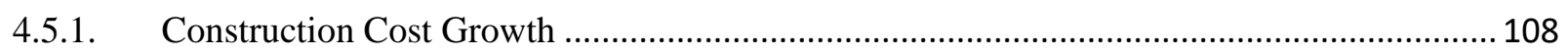

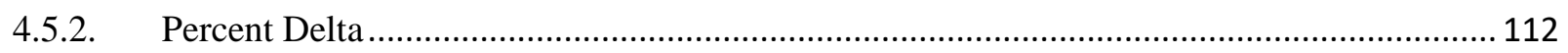

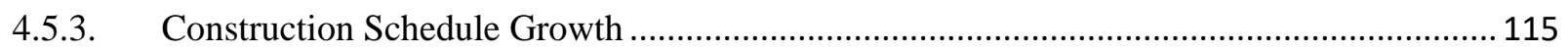

4.5.4. Lost-Time Injuries per Millions of Dollars ................................................................... 118

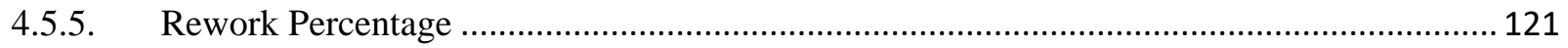

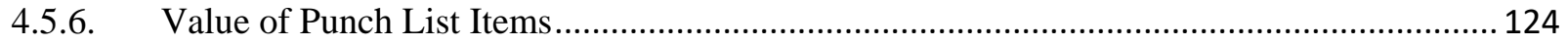

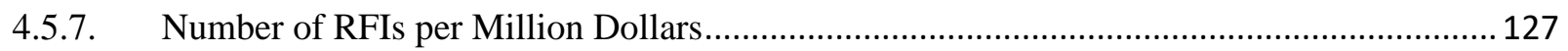

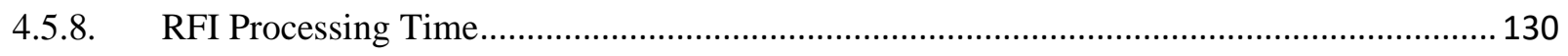

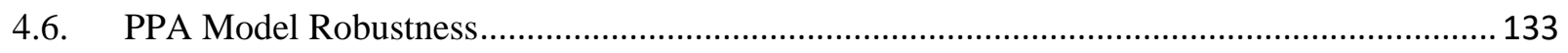

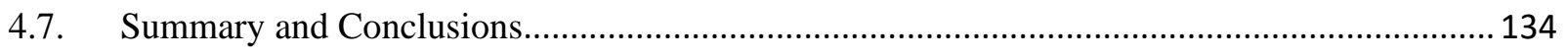

5. Chapter 5 Quantitative Model of Project Competency .......................................................... 136

5.1. Statistical/Mathematical Methodology ……........................................................................ 136

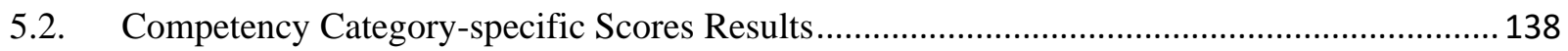

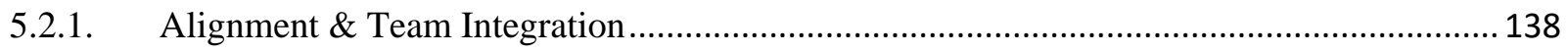

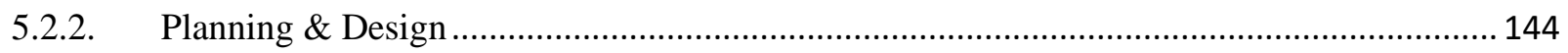

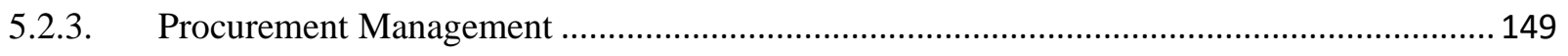

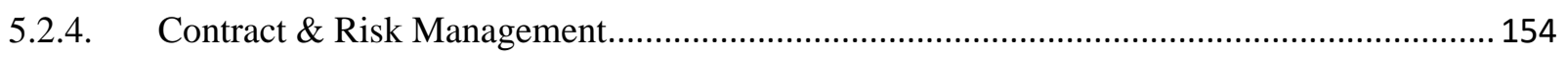

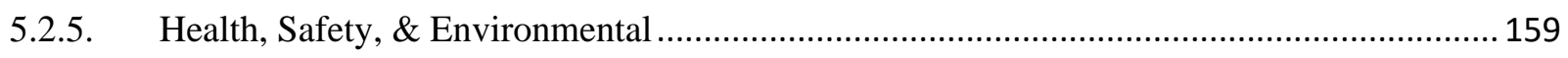

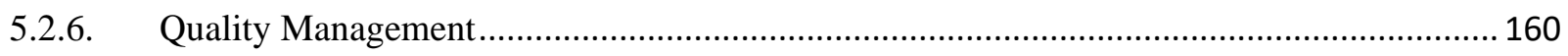

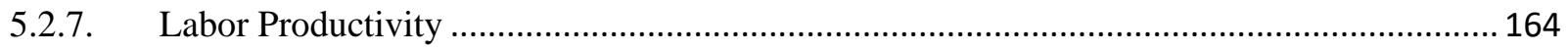

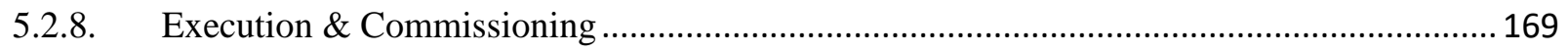

5.2.9. Change Management \& Project Control ........................................................................... 175

5.3. Correlation Matrix between Competency Factors and Performance Metrics ......................... 180

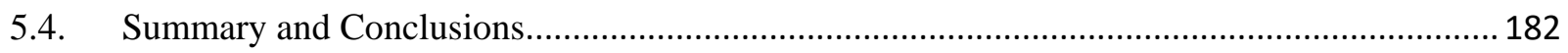

6. Chapter 6 Relationship between Project Competency and Project Performance......................... 183

6.1. Second Mathematical Model Formulation.......................................................................... 183

6.2. Competency Assessment (CCA) Model Results.............................................................. 185 
6.3. Competency Category Assessment (CCA) score vs Project Status ...................................... 188

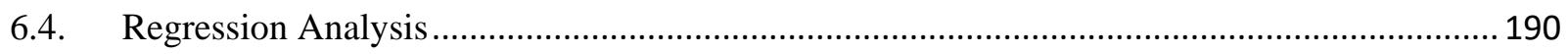

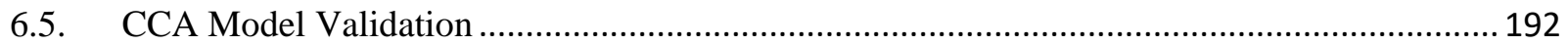

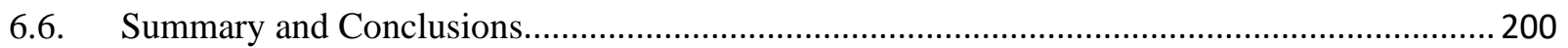

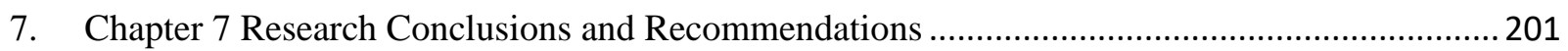

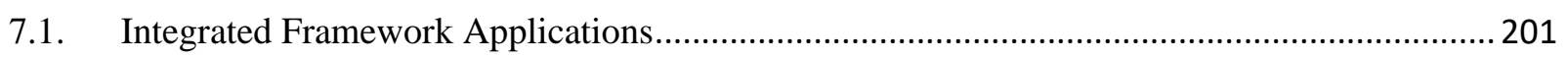

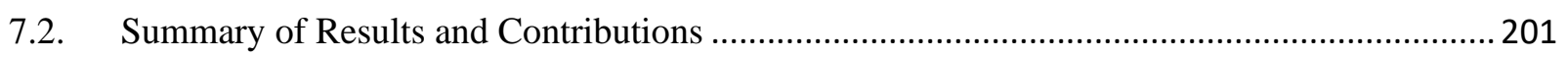

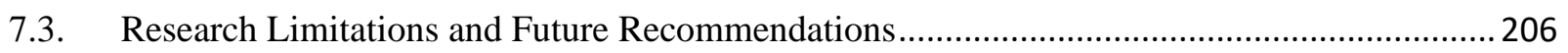

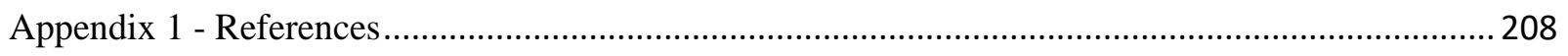




\section{List of Figures}

Figure 1: Value added of the construction industry as a share of the U.S.' GDP from 2007 to $2018 \ldots . . . .19$

Figure 2: Forecast for new construction put in place in the U.S. from 2011 to 2023 (in billion USD)...... 20

Figure 3: Construction spending in the U.S. from 2011 to 2018, by sector...........................................20

Figure 4: Manufacturing vs. Construction Industry (McKinsey 2015) ............................................... 21

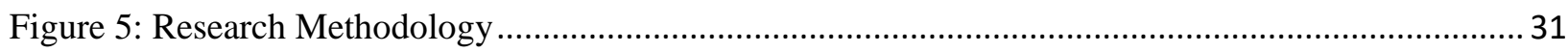

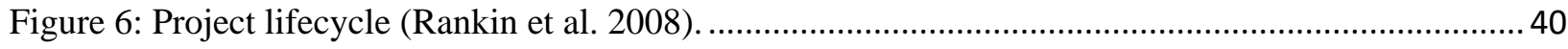

Figure 7: Proposed construction project competencies hierarchy .......................................................... 56

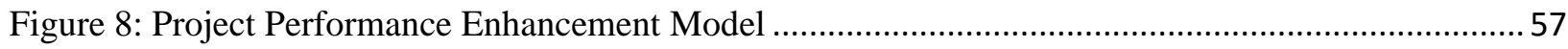

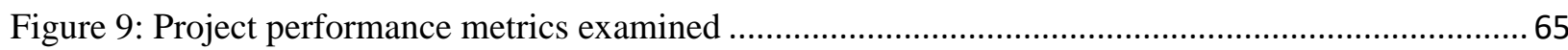

Figure 10: Summary of the competency categories across the project life cycle ................................... 71

Figure 11: Geographic distribution of studied projects................................................................. 78

Figure 12: Respondent's roles in studied projects ......................................................................... 79

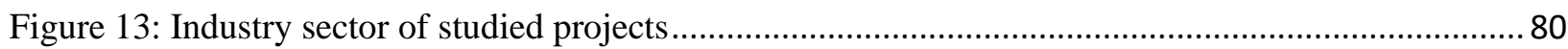

Figure 14: Construction work type of studied projects ................................................................. 81

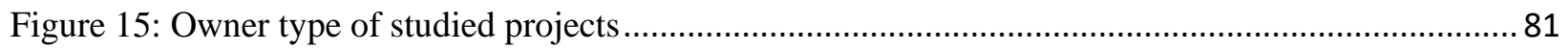

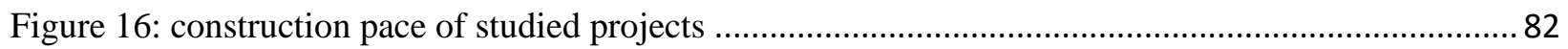

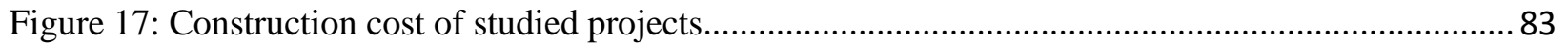

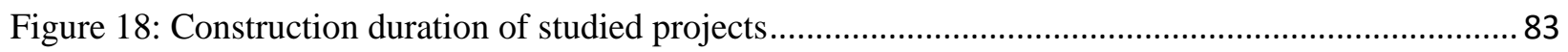

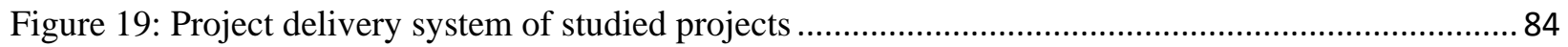

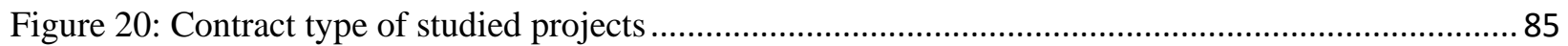

Figure 21: Correlation Matrix for Performance Metrics ...................................................................... 88

Figure 22: Weights breakdown of the PPA metrics ........................................................................ 93

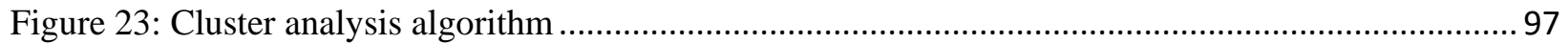

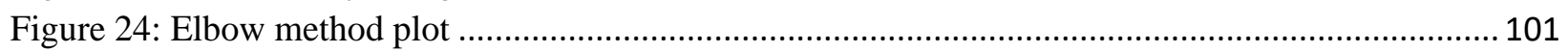

Figure 25: Average silhouette method plot.................................................................................. 103

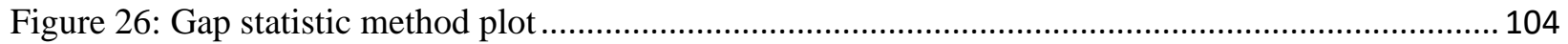

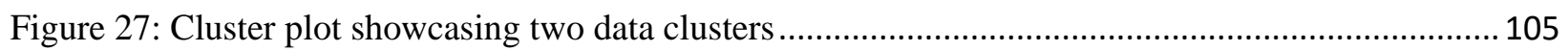

Figure 28: Q-Q plot of project performance assessment score ........................................................ 106

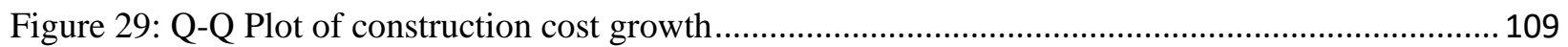

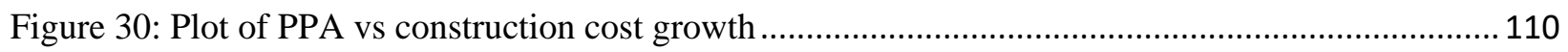

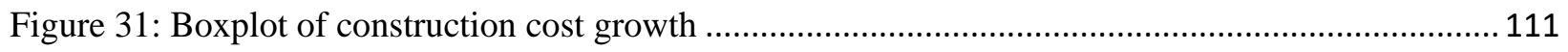

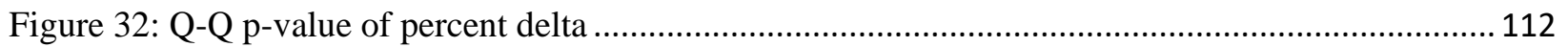

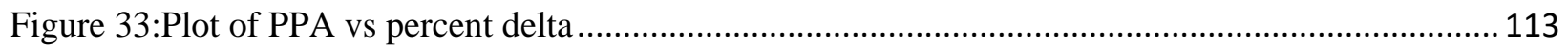

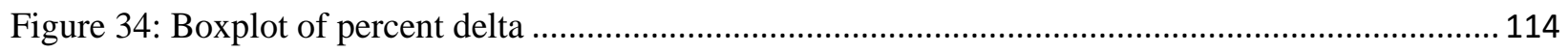

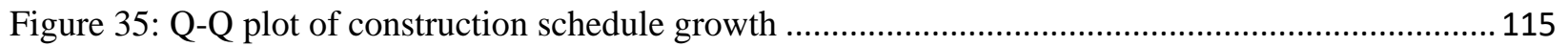

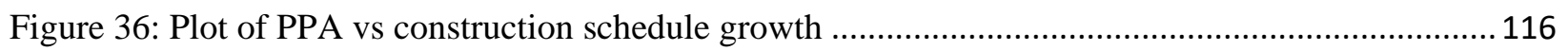

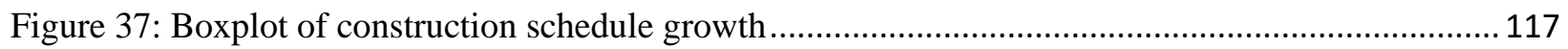

Figure 38: Q-Q plot of lost-time injuries per millions of dollars ......................................................... 118

Figure 39: Plot of PPA vs lost-time injuries per millions of dollars ................................................... 119

Figure 40: Boxplot of lost-time injuries per millions of dollars ........................................................ 120

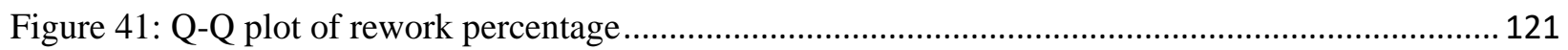

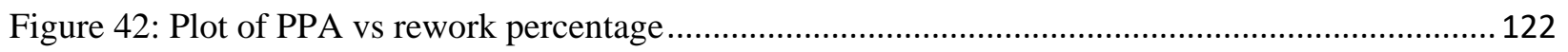

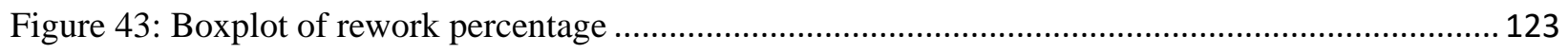




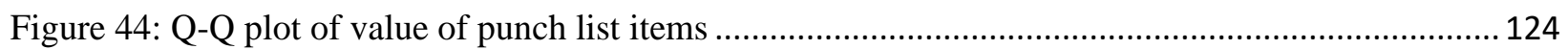

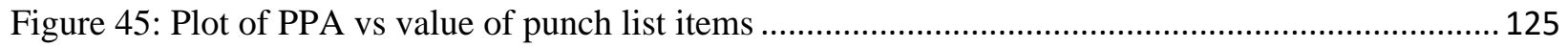

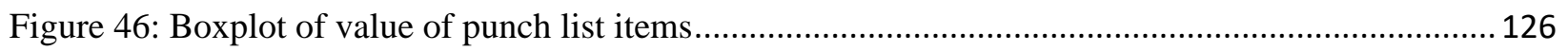

Figure 47: Q-Q Plot of number of RFIs per million dollars ......................................................... 127

Figure 48: Plot of PPA vs number of RFIs per million dollars........................................................ 128

Figure 49: Boxplot of number of RFIs per million dollars ............................................................... 129

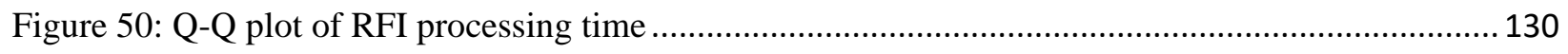

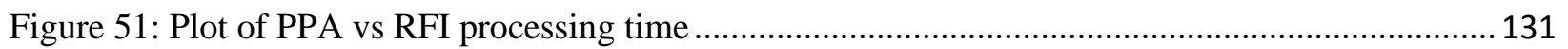

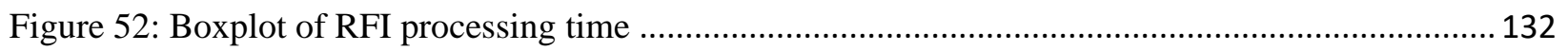

Figure 53: Maximum and minimum deviations of the PPA weights .................................................. 133

Figure 54: Correlation matrix for Alignment \& Team Integration factors ............................................. 139

Figure 55: Weights of subcategories under Alignment \& Team Integration factors .............................. 140

Figure 56: Q-Q Plot of Alignment \& Team Integration score .......................................................... 141

Figure 57: Boxplot of Alignment \& Team Integration score.......................................................... 143

Figure 58: Correlation matrix for Planning \& Design factors ............................................................. 144

Figure 59: Weights of subcategories under Planning \& Design factors ............................................... 145

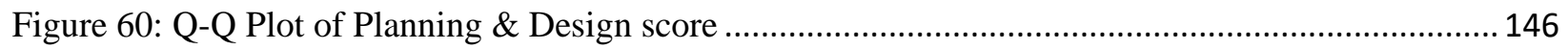

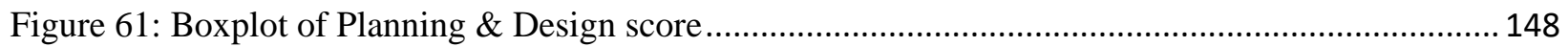

Figure 62: Correlation matrix for Procurement Management factors...................................................... 149

Figure 63: Weights of Subcategories under Procurement Management factors ..................................... 150

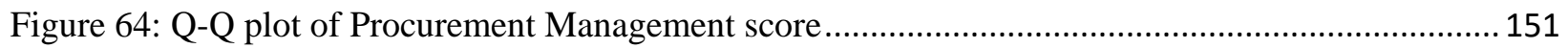

Figure 65: Boxplot of Procurement Management score .................................................................... 152

Figure 66: Q-Q plot of Contract \& Risk Management score ............................................................ 156

Figure 67: Boxplot of Contract \& Risk Management score .............................................................. 158

Figure 68: Correlation matrix for Health, Safety, \& Environmental factors ......................................... 159

Figure 69: Correlation matrix for Quality Management factors ....................................................... 160

Figure 70: Weights of subcategories under Quality Management factors .............................................. 161

Figure 71: Q-Q plot of Quality Management score ........................................................................ 162

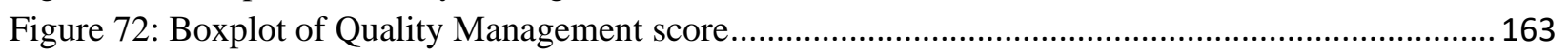

Figure 73: Correlation matrix for Labor Productivity factors............................................................. 165

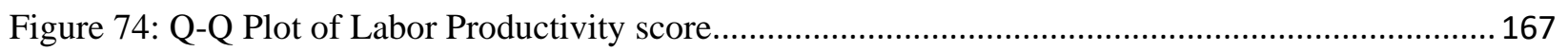

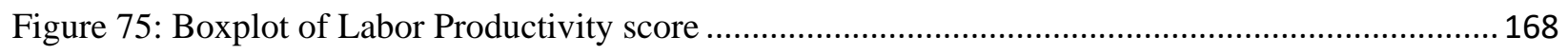

Figure 76: Correlation matrix for Execution \& Commissioning factors ................................................. 170

Figure 77: Weights of subcategories under Execution \& Commissioning factors ................................. 171

Figure 78: Q-Q plot of Execution \& Commissioning score ........................................................... 172

Figure 79: Boxplot of Execution \& Commissioning score ................................................................. 173

Figure 80: Correlation matrix for Change Management \& Project Control factors ............................... 175

Figure 81: Weights of subcategories under Change Management \& Project Control factors .................. 176

Figure 82: Boxplot of Change Management \& Project Control score................................................. 179

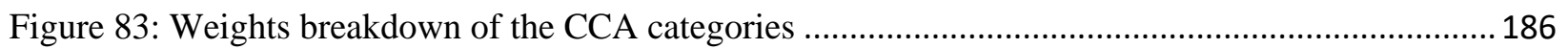

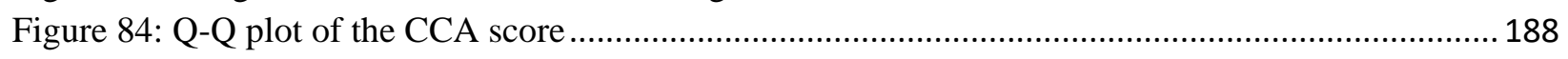

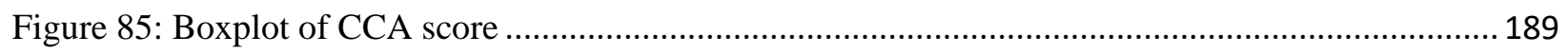

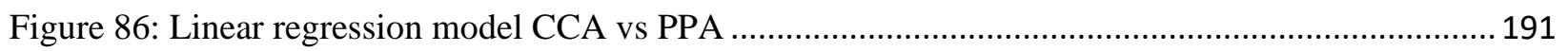

Figure 87: Checking the regression assumption ............................................................................. 192 
Figure 88: Roadmap for practitioners and researchers

201

\section{List of Tables}

Table 1: A summary breakdown of the performance metrics across nine performance areas (El Asmar et

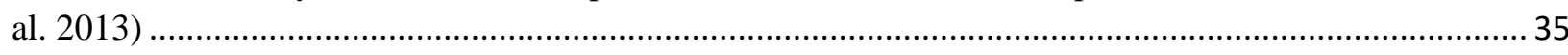

Table 2: A summary of the performance metrics (Carpenter and Bausman 2016)................................. 36

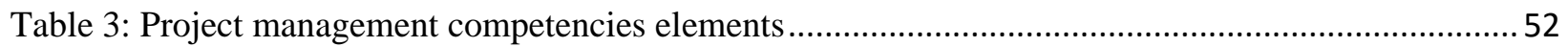

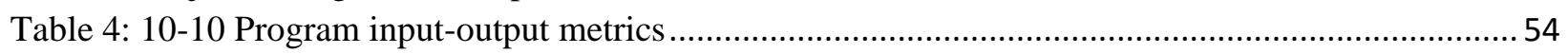

Table 5: Sample performance indicators for construction projects..................................................... 56

Table 6: Summary of previous research in project competencies/performances and their relationships ... 59

Table 7: Range of values for performance metrics for the studied projects ..........................................69

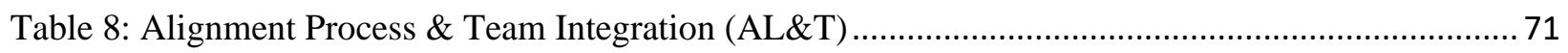

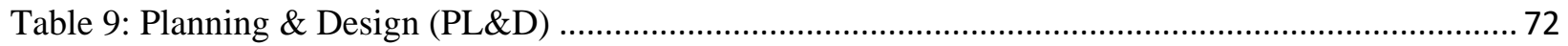

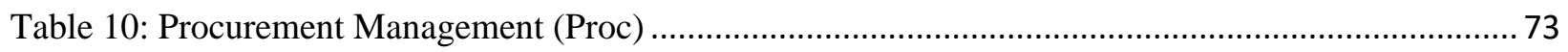

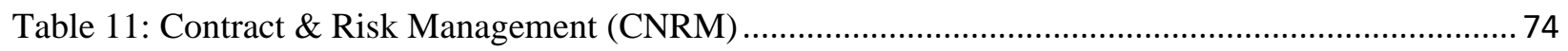

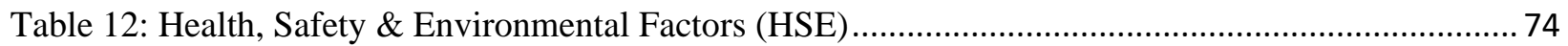

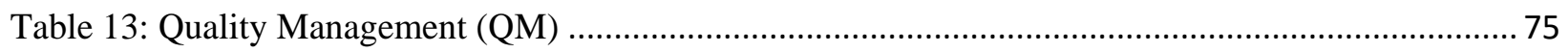

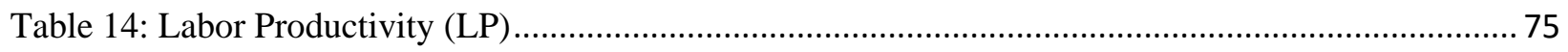

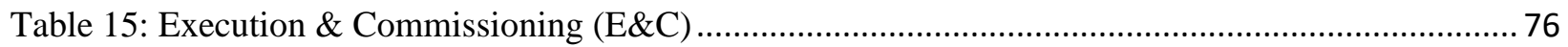

Table 16: Change Management \& Project Control (CMPC) ............................................................... 77

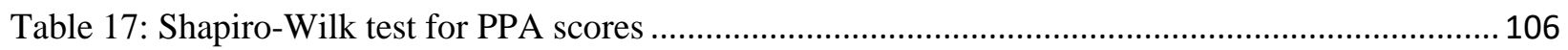

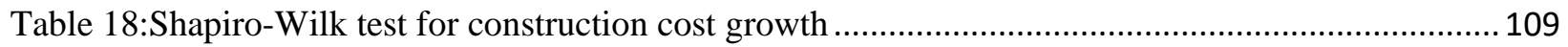

Table 19: Descriptive statistics for construction cost growth .......................................................... 111

Table 20: Shapiro-Wilk Test for percent delta............................................................................. 112

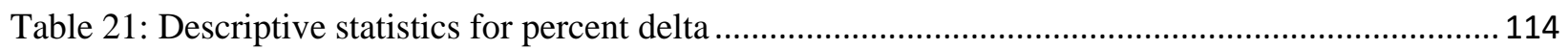

Table 22: Shapiro-Wilk test for construction schedule growth.......................................................... 115

Table 23: Descriptive statistics for construction schedule growth.................................................... 117

Table 24: Shapiro-Wilk test for lost-time injuries per millions of dollars ............................................118

Table 25: Descriptive statistics for lost-time injuries per millions of dollars .......................................... 120

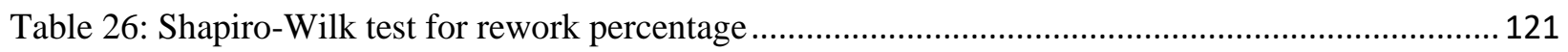

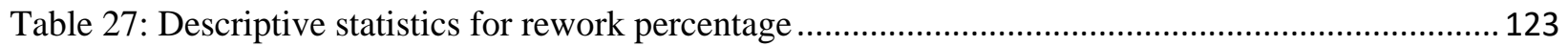

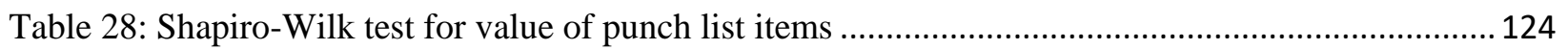

Table 29: Descriptive statistics for value of punch list items ............................................................ 126

Table 30: Shapiro-Wilk test for number of RFIs per million dollars.................................................. 127

Table 31: Descriptive statistics for number of RFIs per million dollars ............................................... 129

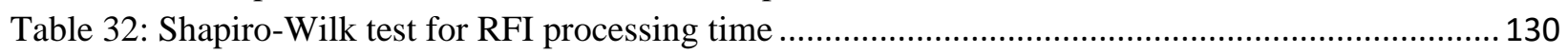

Table 33: Descriptive statistics for RFI processing time ................................................................ 132

Table 34: Maximum, Minimum and Total Deviations of the PPA Weights........................................... 133

Table 35: Correlation of Alignment \& Team Integration factors vs PPA score ..................................... 140

Table 36: Shapiro-Wilk test for Alignment \& Team Integration score ................................................. 141

Table 37: Descriptive statistics for Alignment \& Team Integration score ........................................... 143

Table 38: Correlation of Planning \& Design factors vs PPA score ..................................................... 145

Table 39: Shapiro-Wilk test for Planning \& Design score ............................................................... 147

Table 40: Descriptive statistics for Planning \& Design score............................................................... 148 
Table 41: Correlation of Procurement Management factors vs PPA score............................................. 150

Table 42: Shapiro-Wilk test for Procurement Management score ........................................................... 151

Table 43: Descriptive statistics for Procurement Management score .................................................... 153

Table 44: Correlation of Contract \& Risk Management Factors vs PPA score ...................................... 155

Table 45: Shapiro-Wilk test for Contract \& Risk Management score .................................................... 157

Table 46: Descriptive statistics for Contract \& Risk Management score .............................................. 158

Table 47: Correlation of Quality Management factors vs PPA score ................................................... 161

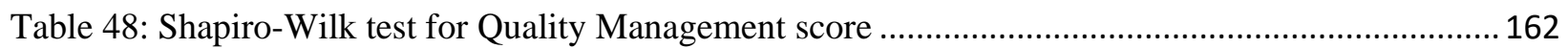

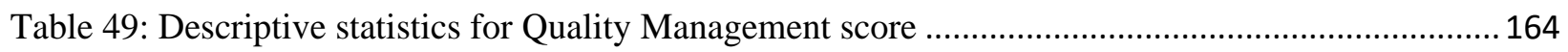

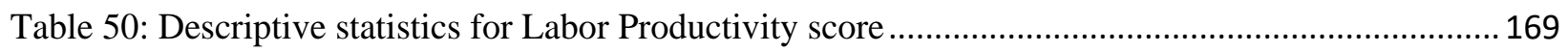

Table 51: Correlation of Execution \& Commissioning factors vs PPA score ........................................ 170

Table 52: Shapiro-Wilk test for Execution \& Commissioning score.................................................... 172

Table 53: Descriptive statistics for Execution \& Commissioning score................................................. 174

Table 54: Correlation of Change Management \& Project Control factors vs PPA score .........................176

Table 55: Shapiro-Wilk test for Change Management \& Project Control score .................................... 178

Table 56: Descriptive statistics for Change Management \& Project Control score................................. 179

Table 57: Correlation matrix between competency factors and performance metrics............................. 181

Table 58: Shapiro-Wilk Test for Competency Category Assessment Score ........................................ 188

Table 59: Descriptive Statistics for Competency Category Assessment Score ........................................ 190

Table 60: Second Mathematical Model Validation ........................................................................... 193

Table 61: Confusion Matrix of Actual vs Predicted Project Statuses for Training Datasets ....................197

Table 62: New projects introduced to second mathematical model...................................................... 198

Table 63: Confusion Matrix of Actual VS Predicted Project Statuses for testing datasets....................... 200 


\section{Chapter 1 Introduction}

Today, construction companies measure their performance by how well a construction project delivers relative to their intended goals. The performance of construction projects is governed by merging many events and interactions with different participants and processes in a constantly changing environment (Sanvido et al. 1992). Many such occurrences and interactions are defined by Spencer and Spencer (1993) as "competencies". These competencies are the inputs that can be quantified and used to distinguish between the poorest-performing and best-performing projects (Omar 2016).

The relationship between construction projects' competencies and performance continues to be a research area that requires broad exploration (Levenson et al. 2006; Isik et al. 2009). Furthermore, this topic exploration is crucial because defining the comparative impact of different project competencies on project performance measures will enable construction practitioners to highlight areas of improvement and make comprehensive recommendations to advance project performance (Antonacopoulou and FitzGerald 1996). In addition, the capacity to recognize and improve the critical competencies of projects that influence project efficiency is anticipated to advance construction organizations' competitiveness and profitability (Fayek 2012).

The scope of this current research is to comprehensively investigate project competencies as leading indicators of project performance that occur among and across different project phases. To fulfill this objective, two integrated mathematical frameworks serve as a robust, accurate, and data-driven weighting technique. The developed mathematical frameworks reduce the subjectivity/uncertainty of the derived relative impacts (weights) of the project competency factors and performance metrics because they are based on actual project data rather than experts' judgment. This study will allow construction practitioners to comprehensively assess both their 
project's competencies and performance using precise mathematics. Thus, the results will also assist construction companies in directing their efforts toward accurately identifying areas of improvement in training and development.

\subsection{Delivering Value in a Demanding Environment}

The well-being of the worldwide economy can often be ascertained by examining the pulse of the engineering and construction $(\mathrm{E} \& \mathrm{C})$ industry. Within most countries, the construction industry is seen as a commodity-producing sector. As such, for many countries, it generally ranks among the top 20 contributors to their Gross Domestic Product (GDP). For example, the construction industries in the U.S have made a noteworthy impact on the economy over the past few years. As shown in Figure 1, the value added of the construction industry as a share of the GDP in the U.S. has significantly increased from 2007 to 2018. In 2018, $4.1 \%$ of the U.S.'GDP led to the value added of the construction sector (Statista 2018). The value added is based on the industry's enhancement in a certain product or service, before being offered to its customers. It occurs when a product gets a new feature that increases the product's value. 


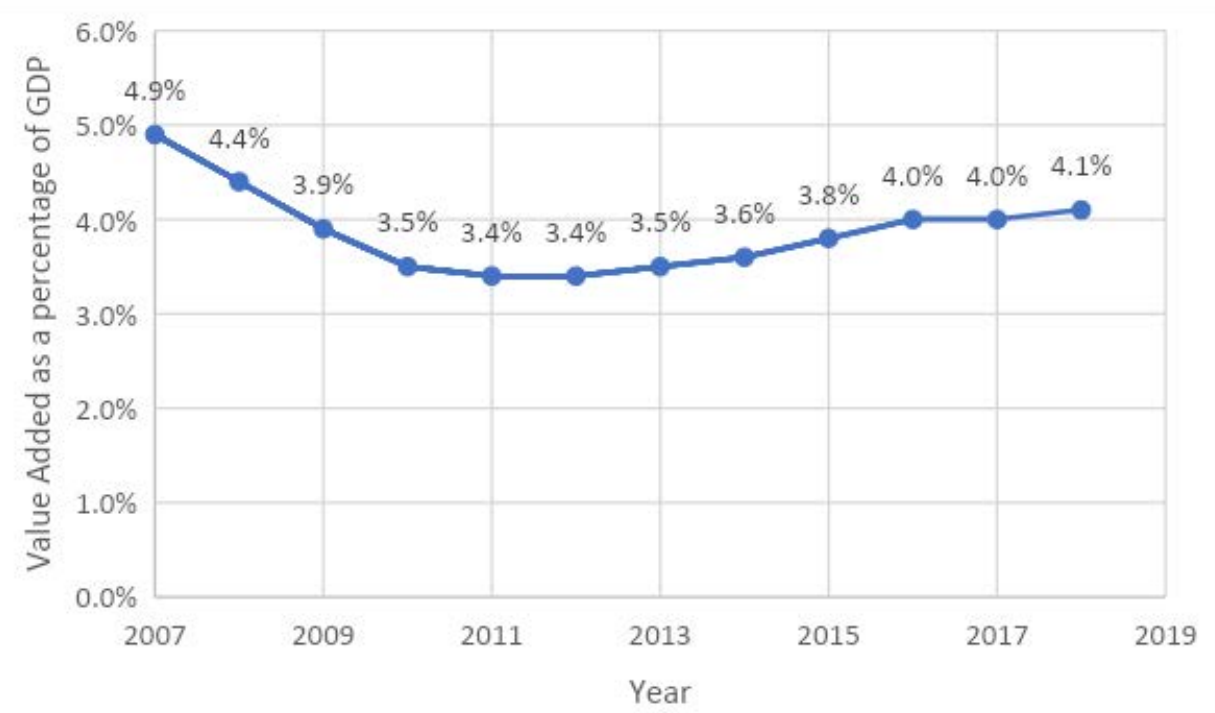

Figure 1: Value added of the construction industry as a share of the U.S.' GDP from 2007 to 2018

Construction in the U.S. is one of the world's biggest market, with expenditure in 2018 at around 1.29 trillion USD, up from around 788 billion USD in 2011, when construction spending reached its lowest level in a decade after the recession (Statista 2018). Figure 2 shows that the industry is on track to continue its positive trend and provides a forecast for total construction put in place in the U.S. between 2011 and 2023. Construction spending is projected to exceed 1.45 trillion USD in 2023, which shows the importance of consistently working to improve this industry to sustain such growth. 


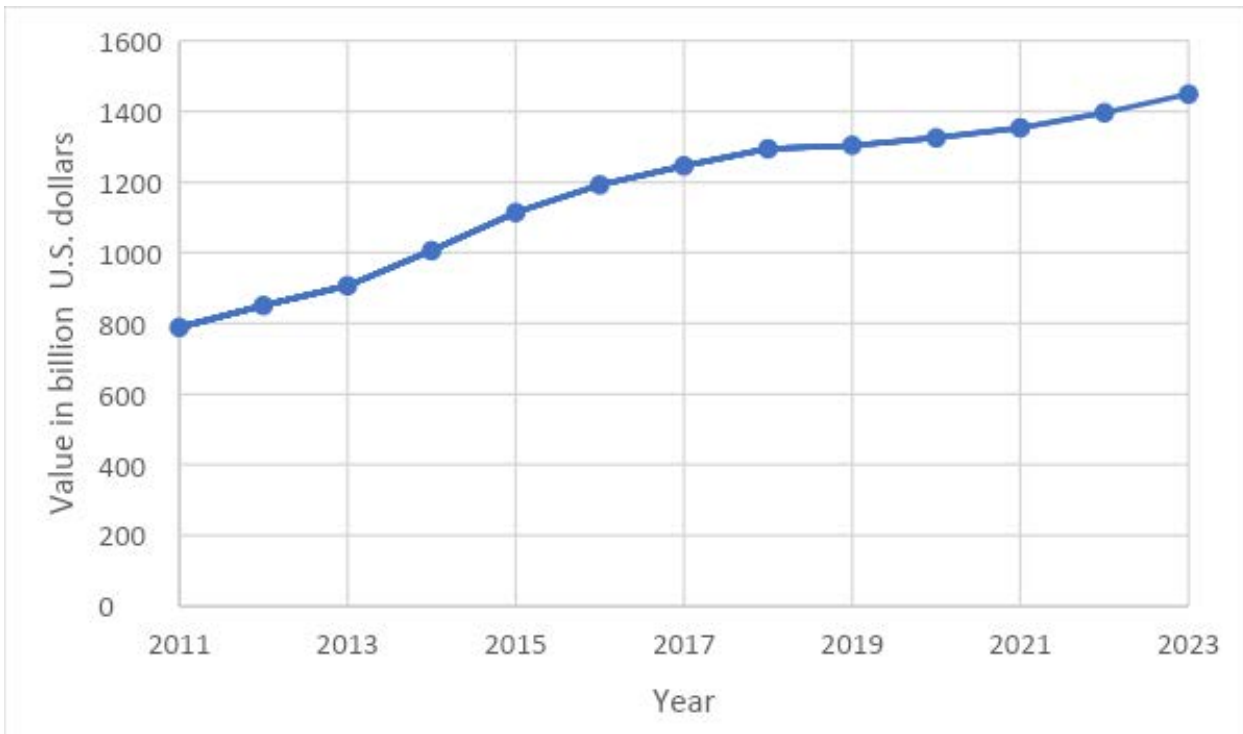

Figure 2: Forecast for new construction put in place in the U.S. from 2011 to 2023 (in billion USD)

The statistics also present the total value of both public and private sector construction spending in the U.S. between 2011 and 2018 as shown in Figure 3. In 2018, private sector construction spending is estimated to have totaled around 992 billion USD compared to the public sector with total construction spending around 302 billion USD (Statista 2018).

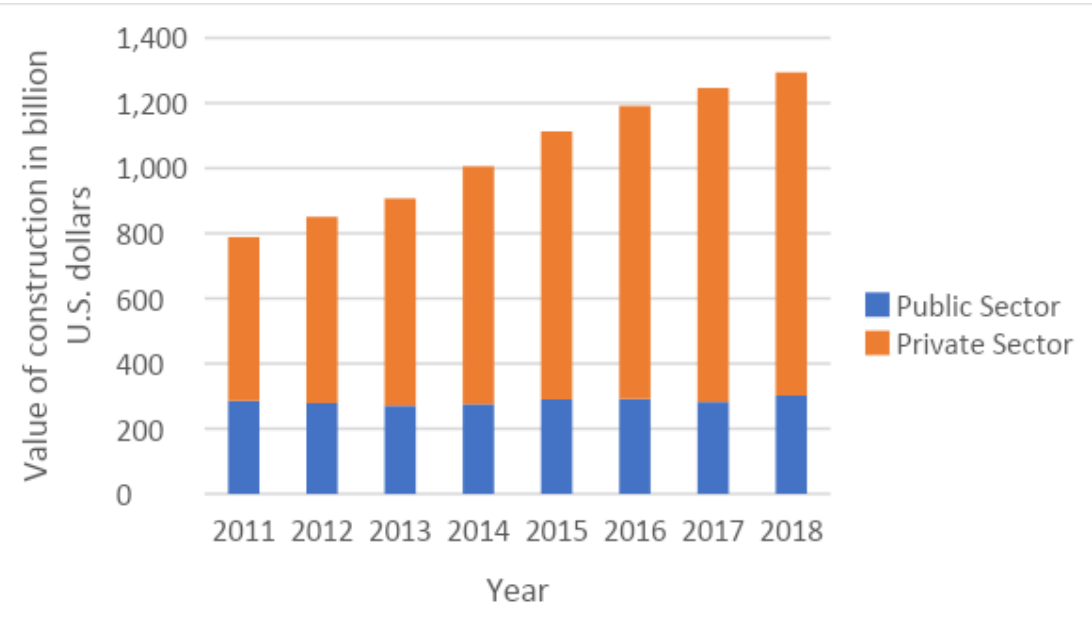

Figure 3: Construction spending in the U.S. from 2011 to 2018, by sector 
While the construction industry is a major factor in the prosperity of countries, especially the U.S., it is fraught with waste and inefficiencies. The information-intensive nature of construction projects is a significant contributor to inefficiencies and losses in the industry. For this reason, the E\&C industry has been facing significant adversity from stagnating or declining productivity. Construction productivity has fallen by more than $30 \%$ since 1960 , while other industries such as auto, aerospace, medical industries, and manufacturing have doubled their productivity during the same timeframe (Teicholz 2013; Hanna 2010). Figure 4 shows the stark contrast between manufacturing (in orange) and construction (in blue). Since 1996, construction productivity has made little to no improvement, while manufacturing has nearly doubled (1.7x). The intensity of the declining construction productivity is most obvious in the case of megaprojects (i.e. projects worth 1 billion USD or more) (Hanna et al. 2018). A study conducted by Changali et al. (2015) revealed that $98 \%$ of megaprojects experience $80 \%$ cost overruns and 20 -month slippage schedules. Another study from Barbosa et al. (2017) highlighted that poor productivity occurring across the construction projects caused an average annual loss of 1.6 trillion USD to the worldwide economy.

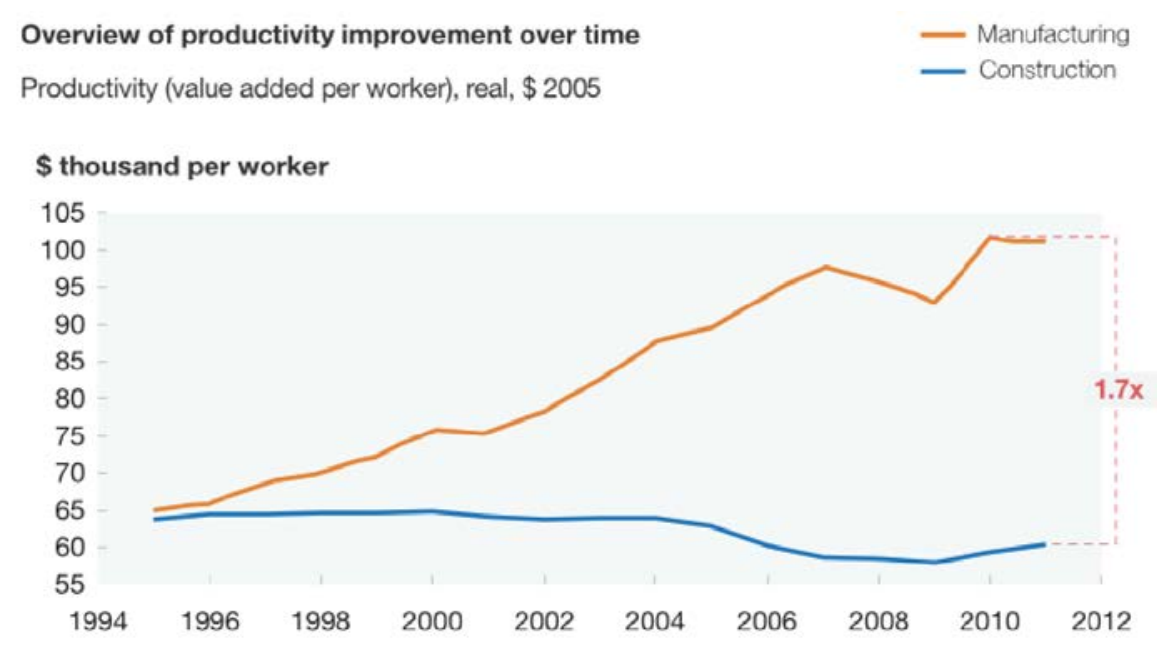

Figure 4: Manufacturing vs. Construction Industry (McKinsey 2015) 
The results of increasing construction productivity are vital for economic stability and growth. For instance, LePatner (2008) highlighted that a one-time improvement of $10 \%$ in construction productivity could produce enough direct economic growth to stabilize Social Security in the U.S. Typically, a substantial part of the effort to enhance the productivity and competitiveness of economies has been directed towards generating more robust and efficient construction sectors, as they were traditionally underperforming relative to other industries (Kagioglou et al. 2001; Lee et al. 2000; Smith 2001; Yang et al. 2010). Higher productivity implies enhanced construction efficiency and, in turn, leads to safer structures, better infrastructure, more sustainable equipment, reduced housing costs, reduced taxes, lower rents, reduced consumer prices, enhanced corporate profits, and a demand for new and better buildings-stimulating the overall economy (Grebler 1973).

\subsection{Business Imperatives: Improving Project Management}

Organizations are more likely to nurture a culture of project management when they fully understand the value it brings and how projects and programs drive change. These organizations also understand that when projects fail, so do profits, because organizations are less likely to achieve strategic goals. A study conducted by (PMI 2015) provided evidence that implementing strategy successfully is inextricably linked to an organization's capability to deliver successful projects. This study showed that high-performing organizations are demonstrating that adhering to proven project, program, and portfolio management practices reduce risks, cuts costs, and improves success rates of projects and programs. This focus on practices emphasizes the need for all organizations to embed a project management mindset in their culture, they will be better able to create a sustainable competitive advantage. High-performing organizations drive project 
management and deploy related competencies with a goal of maximizing organizational value. This same Pulse study from PMI also demonstrated that projects within these organizations meet original goals and business intent two-and-a-half times more often than those in low-performing organizations (90\% vs. 36\%). High-performing organizations also waste about 13 times less money than low performers. The 2015 Pulse study finds that organizations continue wasting 109 million USD for every 1 billion USD invested in projects and programs. But, organizations that embrace, value, and utilize project management—and both recognize and attribute their success to it—report more success, less waste and achieve greater competitive advantage (PMI 2015).

The success of high-performing organizations raises a question: What helps an organization build and sustain its growth capacity to become a high performer? To address this question, the researcher will highlight what high-performing organizations do differently to have successful projects - and how they are creating a competitive advantage by embedding a project management mindset in their projects. Throughout this dissertation, the focus will be on assessing project performance as a key component of project management. It is worth mentioning that for performance measurements to be effective, the measures must be accepted, understood, and owned across the construction organization and its different construction projects. Therefore, eight project performance metrics were identified, spanning six performance areas: cost, schedule, productivity, safety, quality, and communication. High performing organizations embed a project management mindset in their projects by focusing on various competency factors. These competency factors include, but not limited to alignment \& team Integration, planning \& design, procurement management, risk management, health, safety \& environmental, quality management, labor productivity, execution \& commissioning, and change management \& project control. The motive behind investigating project competencies and performance is to establish the relationship between 
project competencies, as leading indicators for measuring project performance, and to identify their effect on project performance.

\subsection{Problem Statement}

The limitations of previous studies concerning project performance assessment can be categorized into five principal limitations: 1) few studies conducted a comprehensive assessment and evaluation of the overall performance in different performance areas; 2) none of the studies captured the different categories of project competencies that occur among and across project phases; 3) none of the studies did a comprehensive assessment and evaluation of the various competencies in different competency categories; 4) the subjective nature of the derived weights of the performance/competency metrics in previous studies, as they were based on experts' opinion; 5) limited investigation of the relationship between the project competencies as leading indicators for project performance improvement and quantifying the relative impact of the different project competencies on project performance. Each of the following paragraphs describes each of the limitations in detail.

Evaluation of project performance has gained significant attention in the construction domain. Within this conversation, one such difficulty is that project management literature review does not provide a coherent nor commonly accepted definition of project success (Chovichien and Nguyen 2013). A project's success could be defined differently depending on the stakeholders, since every individual has a subjective set of success requirements. As such, each notion of success is distinct across individuals and industries (Freeman and Beale 1992; Hartman and Ashrafi 1996). Mills et al. (2003) have observed that determining whether a project is a success is a complex problem and a project cannot always be seen to be totally successful or complete failure, citing the 
previous research (Belassi and Tukel 1996; Pinto and Slevin 1989; Fowler and Walsh 1999). The main challenge in assessing a project's overall performance is that it cannot be done by evaluating a single metric, such as cost; rather, it requires the assessment of several metrics that span across performance areas. Thus, the challenge is to have a comprehensive assessment and evaluation of the overall performance in different performance areas by aggregating the numerous performance metrics, while also considering that each metric has a different unit or units of measurement. In the current construction industry, much effort has been spent on precisely measuring and controlling individual performance metrics (i.e. cost) and determining performance indices for them. However, there have been far fewer comprehensive indices that evaluate project performance across numerous performance metrics.

Previous research focused on either a limited set of project competencies, a single phase of construction, or both without combining the project competencies into an integrated framework to measure project competency level. Most of these studies did not provide a holistic study of the competency level of the projects, since they undermined the fact that the competency factors selection process should capture the main events and interactions that occur throughout the main project phases. Additionally, most of the studies either lacked a quantitative assessment of project competencies or overlooked the unequal contributions of different competencies to the project performance. However, another problem arises from this approach. Competencies can be divided into categories (i.e. Alignment \& Team Integration, Change Management \& project Control Execution \& Commissioning...etc.), and each category has a different number of competencies. Thus, treating all competencies at once would result in overestimating/underestimating the total weight of the competency categories that have a greater/smaller number of factors than others. 
The development of a comprehensive and precise index of project performance requires identifying the relative importance of the different performance metrics that determine project success or the different project competencies that determine an organization's capability to deliver successful projects. It is logical to assume that some performance metrics/competency factors are more impactful on projects than others. Thus, it is essential to determine their relative importance (weights). Previously, the determinations of relative importance have been made by opinions gathered from survey responses and questions posed to industry experts. However, as with any assessment based on opinion, these weights are inherently subjective. The methodology applied in this paper addresses this issue of subjectivity by using actual project data, rather than the subjective judgment of industry experts.

Lastly, previous research has considered, in many situations, how project competencies are a measure of project performance (Fayek 2012). Thus, this research did not investigate project competencies as a prerequisite for project performance, or the fact that project competencies are leading indicators of project performance improvement. In addition, none of the previous studies evaluate the relative impact of the different project competencies on project performance. Therefore, to overcome these limitations of previous research, this thesis develops a comprehensive framework and methodology for evaluating project competencies and performances and identifying their relationship each other.

\subsection{Objective Statements and Research Contribution}

Given the previously stated situation, the academic research objectives presented in this thesis are summarized as follows: 
The objectives of this research were met by using two robust, accurate, and data-driven weighting mathematical frameworks.

1. First mathematical model provides a systematic and structured process to evaluate project performance from the contractor's perspective with respect to a clear set of goals and objectives by creating a comprehensive performance score. This score is named the PPA score and it combines eight key performance metrics spanning over six performance areas: cost, schedule, productivity, safety, quality, and communication.

2. K-means clustering is used to benchmark successful projects from less-than-successful projects. K-means clustering is one of the most used unsupervised machine learning algorithms used to group a set of projects in such a way that projects in the same group (called a cluster) behave similarly to each other. Otherwise, if the difference between a project's performance and the other projects' performances already in the cluster is significant, then the project is assigned to a different cluster.

3. This project identifies various competency factors spanning nine categories: alignment \& team Integration, planning \& design, procurement management, risk management, health, safety \& environmental, quality management, labor productivity, execution \& commissioning, and change management \& project control. Next, two steps statistical analysis is performed; the first step is to do pairwise correlation, which is used to eliminate the problem of multicollinearity and the second step is to investigate the relationship between each of the competency factors as a stand-alone factor and the PPA score to determine the top competency factors that impact the PPA score.

4. First mathematical model can also be used by practitioners and researchers to compute a customized competency category-specific score from various competency factors that are 
inputs from academia and industry practitioners. In this way, a fair comparison for all the competencies is affirmed and it eliminates the overestimating/underestimating problems of the total weight of the competency categories that have a greater/smaller number of factors than others.

5. Second mathematical model is used to quantitatively assess the relative contribution of the different competency categories to the PPA score and provide one unique comprehensive score named the Competency Category Assessment (CCA) Score.

6. A regression model was developed to examine the degree of association between the CCA score and the PPA score, which can be used by construction practitioners to predict the overall performance score of a project at any point in its timeline.

It is important to note that the developed mathematical frameworks reduce the subjectivity/uncertainty of the derived relative impacts (weights) of the project competency factors and the performance metrics, as they are based on actual project data rather than experts' judgment.

\subsection{Research Methodology}

The research was divided into four distinct phases that were implemented in chronological order. The high-level roadmap shown in Figure 5 outlines the methodology used for achieving the research objectives.

Phase 1: is a comprehensive review of available literature concerning project performance and the different metrics used in numerous studies. Further emphasis is placed on the statistical and mathematical techniques used to measure project performance, as well as the development of project success indices. The literature review also highlights the different project competency categories used in previous studies and how each affects the overall project success. Finally, the 
relationship between the different project competencies as leading indicators for project performance is investigated, placing stress on the various methods; either statistical or mathematical techniques used in studying this relationship. After a careful examination of the existing literature, this research highlighted several key gaps in the literature that have not yet been addressed. Chapter 2 will discuss this phase and these gaps in depth.

Phase 2: is a description of the interview process with industry experts to identify the research variables, which can be classified as the project performance metrics and project competency factors. This phase will be discussed in detail in chapter 3 .

Phase 3: is a description of the five stages of the data analysis phase.

First stage: is to use mathematical model 1 to determine the data-driven weights of the eight-performance metrics and finally compute the PPA score and check the model's robustness to assess its reliability and reasonableness. K-means clustering is also performed to benchmark successful projects from less-than-successful projects.

Second stage: is to determine the top competency factors contributing to the PPA score using pairwise correlation and the statistical modeling technique Spearman's Correlation test. Pairwise correlation is used to eliminate the problem of multicollinearity between the different factors. There is no universal cut-off, but, in this dissertation, pairwise correlation is conducted to remove competency factors with correlations coefficients $>0.75$ or $<-0.75$. The second part presents how the statistical modeling technique Spearman's Correlation test is used. It is important to note that this test is used because the independent variables (competency factors) and the dependent variable (the PPA score) are found to be non-normal data, based 
on normality checks (Q-Q plots and Shapiro-Wilk test). Selected competency factors are the ones resulting in a p-value of less than 0.05 , which is sufficient at a 95\% confidence level to reject the null hypothesis that there is no correlation between the competency factor and PPA score.

Third stage: is to use mathematical model 1 to derive the relative impact of the competency factors within its category and use these factors to compute a competency category-specific score.

Fourth stage: is to develop mathematical model 2 to assess the relative contribution of the different competency categories scores to the PPA score and provide one unique comprehensive score of the competency categories, named the Competency Category Assessment (CCA) score.

Fifth stage: is to develop a regression model to examine the degree of association between the CCA score and PPA score and to perform the models' validation to assess their reliability, reasonableness, and the models’ future predictability.

Chapter 4 will discuss the first stage, chapter 5 will discuss the second and third stages, and chapter 6 will discuss the fourth and fifth stages.

Phase 4: will present conclusions and recommendations for future research, which will be discussed in chapter 7. 


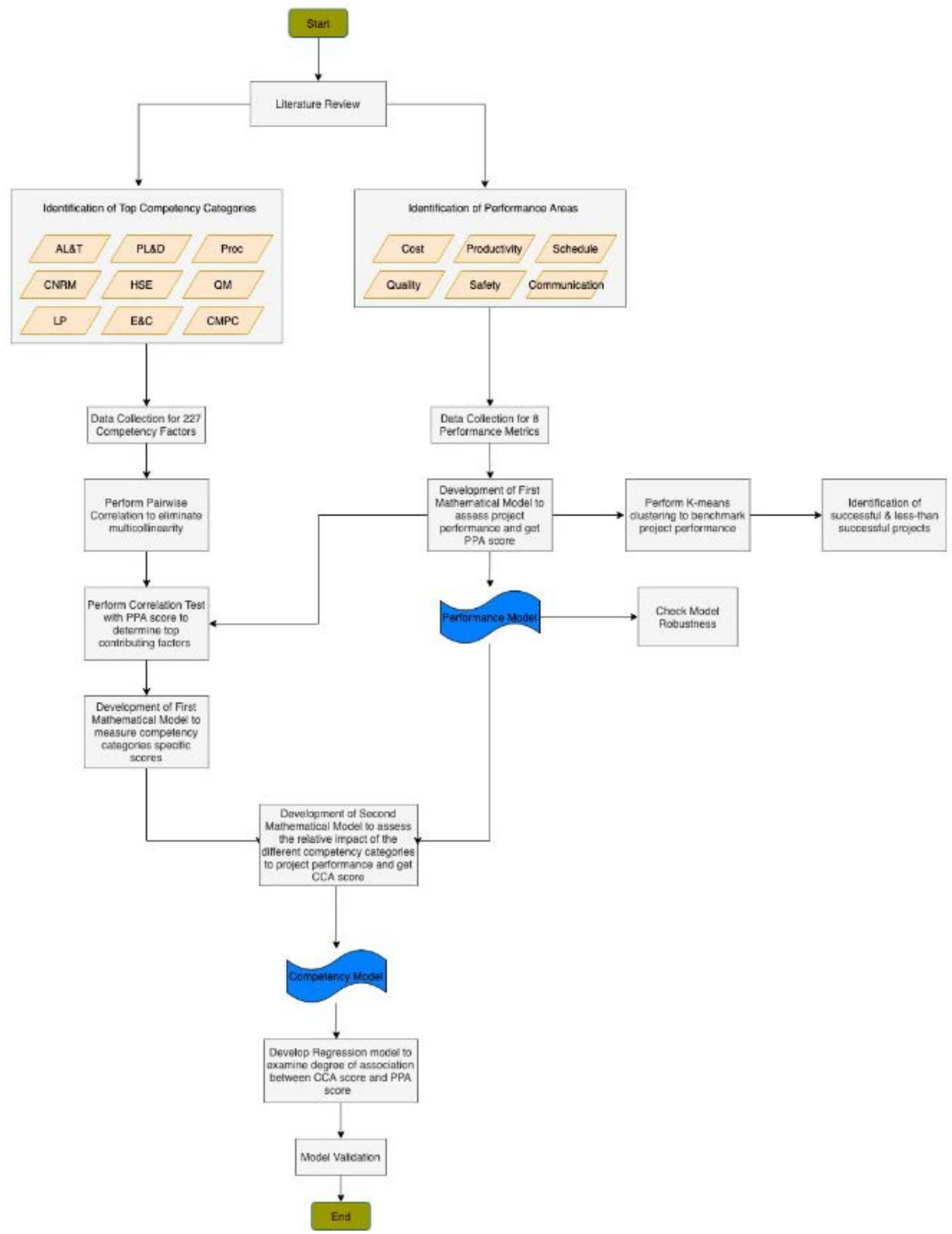

Figure 5: Research Methodology 


\subsection{Dissertation Organization and Structure}

- Chapter 1 consists of an introduction, providing the background and motivation for this study, as well as highlighting the main objectives and methodology for the study.

- Chapter 2 consists of a review of the literature, divided into three main parts:

o Project performance metrics

o Project competency factors

o Relationship between project competencies and project performance This chapter also highlights the research opportunities and the objectives needed to fill gaps in the literature.

- Chapter 3 presents the research methodology, including:

o The process of identifying the research variables

o Data collection

o The characteristics of the collected project data

- Chapter 4 discusses the development of the mathematical formulation called the Project Performance Assessment (PPA) score. This chapter also aims to identify objective databased weights for these metrics; and therefore, alleviate the subjectivity associated with experts' judgment regarding their relative importance to one another. Finally, K-means clustering is used to benchmark successful projects from less-than-successful projects.

- Chapter 5 discusses the development of the mathematical formulation of the competency category-specific scores. The competency category-specific scores incorporate a set of factors with varying degrees of impact (i.e. weights) on project performance.

- Chapter 6 discusses the development of the mathematical formulation called the Competency Category Assessment (CCA) Score. The CCA score incorporates a set of competency category-specific scores (Chapter 5) with varying degrees of impact (i.e. weights) on project performance (PPA score developed in Chapter 4). In this chapter, a regression model was developed to examine the degree of association between the CCA score and the PPA score and perform model validation to assess the reliability, reasonableness, and the model's future predictability.

- Chapter 7 presents the conclusions and recommendations of this study.

- Appendix A contains the bibliography used for this study. 


\section{Chapter 2 Literature Review}

\subsection{Introduction}

Construction projects are completed as a result of merging many events and interactions, with varying participants and processes in a constantly changing environment. Many of these events and interactions can be quantified and then used to distinguish between the poorestperforming and best-performing projects (Omar 2016). Many such occurrences and interactions are defined by Spencer and Spencer (1993) as "competencies". Performance measures, on the other hand, are also vital to construction organizations and projects as they are used to manage business and measure the success of projects (Chan and Chan 2004). Over the past few decades, researchers have shown interest in the areas of project competencies and performance and these findings will be the focus of this chapter.

Defining and measuring different project competencies as leading indicators of project performance, is expected to improve the understanding and identification of requirements for the successful execution of construction projects. A synopsis of previous research is presented in this chapter to identify current research gaps in the areas of project performance, project competencies, and their relationship. This synopsis will provide a basis for defining project competencies and performance measures, their hierarchies, evaluation and, data collection tools.

\subsection{Review of Project Competencies and Project Performance Frameworks}

This chapter presents an overview of previous research in the areas of project performance, project competencies and their relationship to one another. 


\subsubsection{Project Success and Project Performance}

Project success is a key objective for project management and thus its definition and assessment have been topics of long-standing debate. A project management literature review does not provide a coherent nor commonly accepted definition of project success (Chovichien and Nguyen 2013). A project's success could be defined differently by different stakeholders since every individual has a subjective set of success requirements. As such, each notion of success varies across individuals and industries (Freeman and Beale 1992; Hartman and Ashrafi 1996). Mills et al. (2003) have observed that determining a project's success is a complex problem and a project cannot always be seen to be totally successful or a failure, citing the previous research (Belassi and Tukel 1996; Pinto and Slevin 1989; Fowler and Walsh 1999).

Many researchers and professionals have been interested in aspects of projects beyond project success. For instance, budget, schedule, and quality are generally accepted as the primary objectives of a project. Although depending upon the nature of the project, participants, and company level, there are also more specific goals such as safety considerations and stakeholder satisfaction (Chua et al. 1999). Ward et al. (1991) asserted that the quality of relations between participants and the flexibility to integrate modifications should be included as other variables, which may influence client satisfaction and thus impact project success or failure.

Rankin et al. (2008) combined performance metrics for both the construction phase and an extended timeline of building life. In doing so, they covered seven performance areas: cost, time, scope, quality, safety, labor productivity, innovation, and sustainability. Based on the results and industry feedback, additional metrics for practices related to project planning, materials management, and construction supervisory skills development were developed. 
El Asmar et al. (2013) evaluated the performance of IPD projects compared to projects delivered using the more traditional design-bid-build, design-build, and construction management at-risk systems. They gathered quantitative performance data from 35 completed projects. This study considered 34 performance metrics across nine performance areas: cost, schedule, quality, safety, change management, communication, labor, recycling, and business performance.

Table 1 shows a summary breakdown of the performance metrics across these nine performance areas.

Table 1: A summary breakdown of the performance metrics across nine performance areas (El Asmar et al. 2013)

\begin{tabular}{|c|c|}
\hline Performance Area & Performance Metrics \\
\hline Cost & $\begin{array}{ll}\text { - } & \text { Unit cost } \\
\text { - } & \text { Construction cost growth } \\
\end{array}$ \\
\hline Schedule & $\begin{array}{ll}\text { - } & \text { Construction speed } \\
\text { - } & \text { Delivery speed } \\
\text { - } & \text { Construction schedule growth } \\
\end{array}$ \\
\hline Quality & $\begin{array}{l}\text { - } \text { Quality of major building systems } \\
\text { - } \quad \text { Number of deficiency issues } \\
\text { - } \text { Number of punch list items } \\
\text { - } \text { Cost of punch list items } \\
\text { - } \text { Cost of warranty } \\
\text { - } \quad \text { Cost of latent defects. }\end{array}$ \\
\hline Safety & $\begin{array}{ll}\text { - } & \text { Number of OSHA recordables } \\
\text { - } & \text { Number of lost-time-injuries (LTI) } \\
\text { - } & \text { Number of fatalities } \\
\end{array}$ \\
\hline Change Management & $\begin{array}{ll}\text { - } & \text { Total percent of change } \\
\text { - } & \text { Design changes } \\
\text { - } & \text { Design coordination } \\
\text { - } & \text { Average change order processing time, } \\
\end{array}$ \\
\hline Communication & $\begin{array}{ll}\text { - } & \text { Number of RFIS } \\
\text { - } & \text { RFI processing time } \\
\text { - } & \text { Rework } \\
\text { - } & \text { Resubmittals } \\
\text { - } & \text { Claims }\end{array}$ \\
\hline
\end{tabular}




\begin{tabular}{|l|l|}
\hline \multirow{2}{*}{ Labor } & $\bullet$ Overtime \\
& $\bullet$ Second shift work \\
& $\bullet$ Over-manning \\
& $\bullet \quad$ Trend of Percent Plan Complete (PPC) \\
\hline \multirow{2}{*}{ Recycling } & $\bullet \quad$ Labor factor \\
& $\bullet \quad$ Percentage of waste recycled \\
& $\bullet \quad$ Percentage of waste sent to landfills. \\
\hline Business performance & $\bullet \quad$ Overhead \& Profit \\
& $\bullet \quad$ Image \& Return Business \\
\hline
\end{tabular}

In another study, Carpenter and Bausman (2016) reviewed 137 public school projects in Georgia, Florida, North Carolina, and South Carolina. The study compared the performance of these projects using metrics of cost, schedule, and quality. Table 2 shows a summary of the performance metrics used in this study.

Table 2: A summary of the performance metrics (Carpenter and Bausman 2016)

\begin{tabular}{|c|c|}
\hline Cost Metrics & Time Metrics \\
\hline $\begin{array}{ll}\text { - } & \text { Original construction cost } \\
\text { - } & \text { Original project cost } \\
\text { - } & \text { Final construction cost } \\
\text { - } & \text { Final project cost }(\$) \\
\text { - } & \text { Construction cost growth } \\
\text { - } & \text { Unit cost } \\
\text { - } & \text { Student cost }\end{array}$ & $\begin{array}{ll}\text { - } & \text { Planned construction } \\
\text { - } & \text { Actual construction } \\
\text { - } & \text { Planned project } \\
\text { - } & \text { Actual project } \\
\text { - } & \text { Construction growth }(\%) \\
\text { - } & \text { Project growth }(\%) \\
\text { - } & \text { Construction intensity }\left(\mathrm{m}^{2} /\right. \\
& \text { day }) \\
\text { - } & \text { Construction intensity }(\$ / \text { day })\end{array}$ \\
\hline \multicolumn{2}{|c|}{ Quality Metrics } \\
\hline \multicolumn{2}{|c|}{$\begin{array}{l}\text { - Owner satisfaction ratings of work quality for the overall project } \\
\text { - } \quad \text { Owner satisfaction ratings of the design and construction team's ability } \\
\text { to manage and control the project } \\
\text { Project costs, schedule, and product quality using communication, } \\
\text { collaboration, and cooperation methods } \\
\text { - Number of days between the date of substantial completion and date of } \\
\text { final completion (time to complete all punch list and other } \\
\text { miscellaneous work items) } \\
\text { - } \quad \text { Number of warranty and callback incidents } \\
\text { - } \quad \text { Product and service quality } \\
\text { - } \quad \text { Number of construction claim incidents } \\
\text { - Cost severity (to owner) of construction claims }\end{array}$} \\
\hline
\end{tabular}


Another school-building related study conducted by Shrestha and Fernane (2016) attempted to evaluate the performance of public university building projects. They studied 77 projects completed since 2000 in 10 states. For the study, four cost metrics, three schedule metrics, and four change-order metrics were used. The cost performance metrics are contract award cost growth, design \& construction cost growth, total cost growth, and cost per sq. ft. The schedule performance metrics are design \& construction schedule growth, total schedule growth, and construction intensity. Lastly, the change order performance metrics are the number of design change orders, design change-order cost factors, number of construction change orders, and construction change-order cost factors.

Ibrahim et al. (2018) compared four delivery methods: Integrated Project Delivery (IPD), Design-Build (DB), Construction Management-at-Risk (CMAR), and Design-Bid-Build (DBB) over a set of 109 projects. This study considered 12 performance metrics over 5 performance areas: cost, schedule, quality, safety, communication, and change management. These metrics are as follows: The cost performance metric is cost growth, schedule performance metric is schedule growth, quality metrics included punch-list-items, rework, quality/value-related, and overall systems quality, safety metric is OSHA recordable incidents, communication metrics are the number of Requests for Information (RFIs) per million dollars and RFI processing time, and change management metrics are project percentage change, design-related changes, and change order processing time.

Leon et al. (2018) compared different studies and concluded that there are commonplace key performance indicators (e.g., cost, time, quality), whereas others were frequently repeated (e.g., profitability, safety, environment, team satisfaction, client satisfaction, communication). This study proposed a system dynamics (SD) model that integrates eight construction project 
performance indices to simulate the complexities that exist among these interdependent variables and forecast their dynamics over time. This model focuses on the construction phase of projects under unit price contracts.

\subsubsection{Development of Project Success Indices}

Some studies have developed an integrated model to quantitatively assess project performance using a unified cumulative score. Each of the following paragraphs summarizes the main studies from the previous project performance literature.

Lam et al. (2007) derived a success score for 40 DB projects executed in Hong Kong, from which they derived 11 criteriums of success spanning four success key performance indicators: time, cost, quality, and functionality. The weights of the different key performance indicators were derived using principal component analysis (PCA) using the following project success equation, which is used to compute the final project success scores.

$$
P S I \sim D \& B=0.54 * \text { Time }+0.55 * \text { Cost }+0.47 * \text { Quality }+0.42 * \text { Functionality }
$$

El Asmar et al. (2015) developed the Project Quarterback Rating (PQR) method, which was derived from 23 performance metrics in seven areas: customer relations, safety, schedule, cost, quality, profit, and communication. The weights for each of the seven performance areas were based on 35 industry experts' opinions. The success score computed was used to compare the different delivery methods based on their scores.

$$
P Q R=0.45 * \text { Relations }+0.34 * \text { Safety }+0.31 * \text { Schedule }+0.25 * \text { Budget }+0.23 *
$$

Quality $+0.22 *$ Profit $+0.17 *$ Communication 
Osei-Kyei and Chan (2017) developed a pragmatic model to quantify the success of 120 public-private partnership (PPP) projects in developing countries using a fuzzy synthetic evaluation method. The project success index model developed consists of nine success criteria that were grouped into three major critical success areas for PPP projects in Ghana; these include local development and disputes reduction, profit, and cost and technical specifications. Practitioners in developing countries can use the following success index equation to determine the success levels of their projects in a more practical and objective manner.

Success Index $=0.301 *$ Local development and dispute reduction $+0.347 *$ Profit + $0.352 *$ Cost and technical specifications

The study further highlighted that meeting cost project budget and specification requirements was the most important factor with a weight of $35.2 \%$, followed by profitability and meeting project schedule (34.7\%), and then by the ability to provide local development and minimize disputes (30.1\%).

Labib (2019) developed the Project Performance Index (PPI), which is a mathematical model derived from eight performance metrics that span four performance areas including, schedule, communication, change management, and efficiency. The PPI is a single numeric score, which was used to facilitate the comparison of the performance of 189 Wisconsin State projects that may significantly differ in scope, cost, length of contract, etc. The following equation is used to calculate the PPI score for each project. 
PPI Score $=5.360-1.101 *$ RFI processing time $-0.219 *$ percentage change $-0.241 *$ schedule growth $-0.022 *$ schedule factor $-0.014 *$ \# of RFIS / \$ Million - 0.019* \# of change orders / \$Million $+0.005 *$ delivery speed $+0.011 *$ construction speed

\subsubsection{Project Competency}

According to PMI (2008), “project management is the application of knowledge, skills, tools, and techniques to a broad range of activities in order to meet the requirements of a particular project.” Hence, the competency factors selection process attempts to capture the main events and interactions that occur throughout the main project phases (pre-construction, construction, postconstruction). The pre-construction phase includes planning, design, and tendering stages. The construction phase starts at the end of the tendering phase and the start of construction until the project becomes available for use. Finally, the post-construction phase begins at the defect liability period until the end of the project's lifetime. Project lifecycle (Rankin et al. 2008).Figure 6 shows a project's lifecycle as referenced by Rankin et al. (2008).

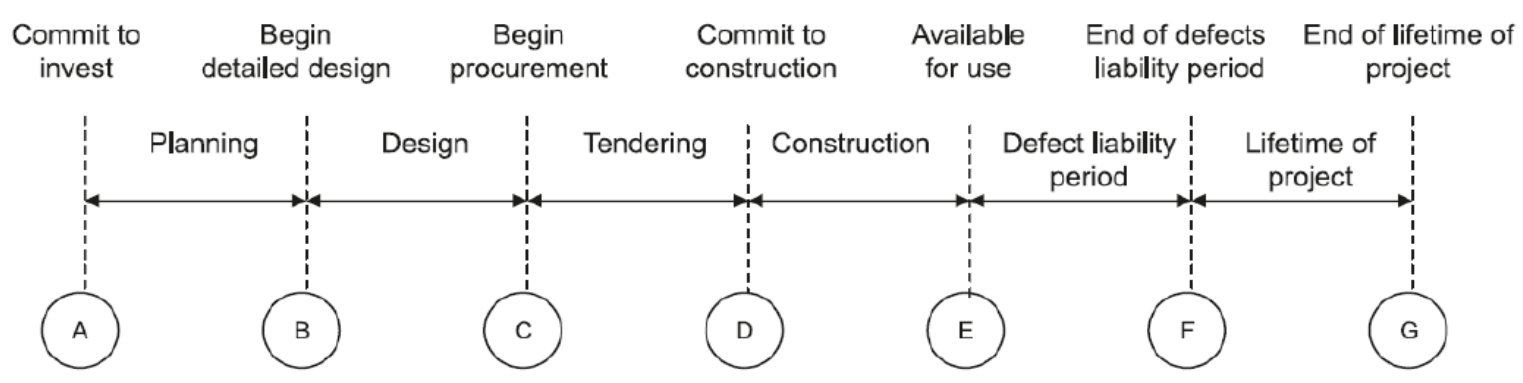

Figure 6: Project lifecycle (Rankin et al. 2008). 
The researcher used the attributes in the former studies as the basis for the project competency in this study. The subsequent sections will elaborate on these attributes and how these attributes can impact project performance.

\subsubsection{Alignment Process \& Team Integration}

Teams, not individuals, are ultimately responsible for the execution of construction projects. Therefore, selecting the appropriate team is a major step in ensuring a successful project. A project team is defined as a group of people with complementary skills, common goals, shared vision, and mutual responsibility for a project (Ward 2008).

Currently, many companies use project teams to deliver products, services, and solve issues, particularly for complicated assignments rather than individual performance issues. The project performance literature has largely remained silent on attitudinal and behavioral results or collectively notes related team outcome factors such as team trust, team cohesion, team efficiency, and team satisfaction in a project setting. The project teams have a purposive structure and consist generally of various team sizes, team compositions, and have a restricted time frame to attain their project objectives (PMI, 2008). Across many studies, project team member alignment and integration have a strong positive influence on project productivity and performance, as suggested by previous scholarship (Dozzi and AbouRizk 1993; Parfitt and Sanvido 1993; Fung and Siow, 2013).

Griffith and Gibson (2001) studied project team alignment during pre-planning and found that factors such as an early definition of project leadership, timely and productive meetings, and effective team building programs were critical to success in this endeavor. A recent study investigated the motivation behind team selection among different delivery systems in transportation projects, and discovered that project experience, schedule availability, and 
professional registration were the top three ranking factors in successful team integration (Bingham et al. 2019).

\subsubsection{2. $\quad$ Planning \& Design}

Over the last few years, researchers have paid increasing attention to the impact of preconstruction planning and design on effective project execution. Pre-construction planning is considered one of the most critical steps for project success. Menches and Hanna (2006b) developed a three-stage preconstruction planning process framework to evaluate the effectiveness of the planning process. Indeed, the effectiveness of a project's planning process can be determined by comparing the planning activities that were performed to those that should have been performed. The framework developed in this study outlines 73 planning activities across three stages: bid preparation planning, pre-construction planning, and jobsite management planning. Among these activities are developing materials storage and staging plan, developing labor and materials tracking reports, forecasting weekly crew requirements, and evaluating and planning crew size. It was also found that a well-performed pre-project plan can contribute to better project performance. Hanna and Skiffington (2010) realized that well-planned projects achieved an average profit margin of $23 \%$ while poorly planned projects had an average profit margin of $-3 \%$. This research also showed that well-planned projects performed better in the areas of contractor satisfaction, budgeted cost, budgeted work hours, quality, relationship with the owner and general contractor, and communication between team members.

On the other hand, engineering and design are critical factors in all future operations of a project. Georgy et al. (2005) presented a study employing multiple attribute utility functions to investigate how project characteristics impact engineering performance. This study also emphasized the negative influence of poor engineering performance on overall project success. 
These negative influences include (1) approximately $10 \%$ of the total cost installed (\$TIC) in the project that can be ascribed to design errors, changes, and omissions, even while construction errors account for only 2\% of the \$TIC (Davis et al. 1989); (2) Post (1998) identified detailed design has been rated by $25 \%$ of facility owners as a weak link in the development of a new plant; and (3) detailed design is the prime cause for change in scope, schedule delays, and field rework (Georgy et al. 2000). It should be the goal of engineering and design professionals to manage changes through effective planning. Barry et al. (2015) investigated the impact of poor design practices on project performance in terms of standard performance metrics such as cost, schedule, quality, safety, and organizational performance. This study identified a comprehensive list of engineering-related factors and then developed an integrated framework to identify engineeringrelated factors that affect construction readiness.

\subsubsection{Procurement Management}

The approach taken by the client to procurement sets the duties and authorities of the whole construction process, which affects the degree to which project partners integrate and cooperate with one another (Love et al. 1998 and Briscoe, et al. 2004). The management of procurement is the key factor contributing to project success and hence client satisfaction. However, previous studies on this subject are limited. The few studies focused on the effects of only one or more procurement-related variables on only some project outcomes. Eriksson (2008a), Eriksson (2008b), Eriksson and Nilsson (2008) developed a systemic and holistic conceptual model regarding clients’ procurement procedures during all phases of the purchasing process, beginning with product design, tendering, bid evaluation, subcontractor selection, payment and ending with the performance assessment. Furthermore, Eriksson and Westerberg (2011) extended on these previous studies to develop a testable holistic procurement framework that further increases 
knowledge about how procurement factors may influence project performance (cost, time, quality, environmental impact, work environment, and innovation). However, none of these studies have examined the relative impact of procurement-related factors on project performance. Although, Gurmu and Aibinu (2017) studied equipment management practices in construction that can potentially improve productivity. They found that effective planning for maintenance and procurement, as well as productivity analysis of equipment used, were the most crucial attributes of successful equipment management.

\subsubsection{Contract \& Risk Management}

Over the past few decades, the global construction industry has been prone to enormous risks related to different activities throughout the project lifecycle that impact project performance (Mbachu and Taylor 2014). Bunni (1985) conducted one of the earlier studies in this field, which included a list of risks and their underlying causes. This list includes (1) physical work, (2) delays and disputes, (3) direction and supervision, (4) damages and injuries, (5) external factors, (6) payment, and (7) laws and arbitrations. The risks are faced by all parties involved in a project. Zou et al. (2010) studied the impact of risk management on an organization's performance and concluded that having a clear risk management plan through defining goals, specifying processes, and optimizing risk allocation reduces costs and improves profitability. Importantly, the relative severity of the risk shared by parties may vary according to the relative importance of their role. Such parties will draft the provisions of the construction contract in their favor, trying to allocate or share the risks among the other contracting parties. Therefore, Peckiene et al. (2013) emphasized the equitable risk allocation between parties in the construction agreement and argued that they should employ comparative game theory, where all the concerns/needs of the contracting parties are addressed. 
The number of claims made in construction projects has significantly increased both in size and number in the last 30 years (Harmon 2003). The evidence of this increase in the U.S. and Canada is very compelling, and includes the following facts: (1) half of claims made requested a $30 \%$ or more increases original contract prices; (2) a third of claims requested $60 \%$ or more increases in original contract prices; and (3) some claim requested amounts very close to the entire original contract price (Cheeks 2003). It is estimated that construction claims in the U.S. cost around \$5 billion per year, and there are no indicators stipulating that such costs are going to decrease with the current practices (Peña-Mora et al. 2002). Globally, the average value of disputes has increased from \$35.1 million to in 2010 to \$46 million in 2016 (Arcadis 2016). Also, the average length for handling disputes has increased from 9.1 months in 2010 to 15.5 months in 2015 (Arcadis 2016).

\subsubsection{Health, Safety \& Environmental Factors}

The construction industry is characterized by the high risk associated with its projects. According to Raheem and Hinze (2014), the construction sector in industrialized countries is responsible for $20-40 \%$ of fatal accidents, although it employs around $10 \%$ of the workforce. The consequences of accidents negatively impact the construction sector by increasing direct and indirect project costs (Ikpe et al. 2012), inducing schedule delays (Han et al. 2014), and adversely affecting workers’ families, companies’ reputations, and society in general (García-Herrero et al. 2012).

Consequently, the safety performance of contractors has become increasingly prioritized and thus one of the important variables influencing companies' decisions to award contracts (Hinze 2013; Saunders et al. 2017). Although many companies try to enhance their safety 
performances considerably by implementing various safety practices and increasing resource allocation by organizations, the control of on-site construction accidents remains a challenge (U.S. Department of Labor 2017). Therefore, making appropriate decisions and taking a proactive approach to integrating an HSSE plan and defining a process for communicating changes to the plan can result in developing strategies to avoid the occurrence of accidents, as well as reducing unnecessary costs in construction (Marle and Vidal 2016).

\subsubsection{Quality Management}

In the construction industry, one pillar of every project delivery process is the quality management control of the materials and services provided. Therefore, during the past three decades, the construction market has significantly increased its demand for quality management programs (Ahire and Golhar 1996). Thus, pressure has been placed on companies to assess the quality of product delivery systems and compete in national and global arenas (Sullivan 2011). Poor quality standards can occur at different project life stages: it can occur during construction, at the intermediate products (outputs of construction activities that are not yet end products) and end products (finished products), and during the operating life of a project (Battikha 2008). Therefore, inspection and testing needs to be performed during all phases of a project starting from materials' delivery, materials storage, on-site fabrication, erection, and upon completion, to ensure that the constructed project and all related components that are handed over to the client comply with the contract requirements (Bubshait and Al-Atiq 1999).

A study from Safapour and Kermanshachi (2019) showed that the implementation of effective quality management strategies led to a relatively low cost for the rework. Taylor and Ford (2006) defined rework as work discovered to require change (either through errors, omissions, or regulation changes). Many large-scale construction projects suffer from the issuance of rework 
that, on average, contributes to $52 \%$ of the total growth of incurred costs and can increase schedule overruns by 22\% (Love 2002). In the literature, rework costs range from 5 to 20\% of the contract value in construction and engineering projects (Love et al. 2018), and changes in the scope of the design account for as much as 50\% of the rework that occurs (Barber et al. 2000; Love and Edwards 2004). Therefore, Safapour and Kermanshachi (2019) identified 51 indicators of manageable rework causes (IMRCs) that span three main categories: organization, project, and people. This study also proposed appropriate construction best practices (BPs) to reduce the cost of manageable and controllable rework in terms of the total cost of a project. These practices were included in the initial list of factors for this research.

\subsubsection{Labor Productivity}

Construction is a labor-intensive industry, where the labor costs represent $30-50 \%$ of the project's total cost (McTague and Jergeas 2002; Hanna 2001). Therefore, the financial success of any construction project requires outstanding labor management and high productivity control (Hanna et al. 2005; Ernzen and Schexnayder 2000). But today there is a shortage of quality and qualified labors in construction markets, Taylor et al. (2016) have revealed that $52 \%$ of the projects surveyed were impacted by qualified labor shortages in his North American construction firm study. The shortage of skilled labor can also result in productivity loss, an increase in prolonged overtime, hourly wage, safety incidents, rework percentages, and schedule overruns, which eventually lead to a project's cost overrun. In fact, the percentage of productive work in a typical construction project ranges between $30-40 \%$, resulting in failure to deliver approximately 50\% of the projects on time and on budget (Hanna, 2010). Thomas et al. (2006) proposed several key principles of workforce management for success that are adopted in this research. These include a defined and fair layoff and termination policy for both crew and project staff, planning for and 
minimizing work disruptions, and having a thorough onboarding system to orient new hires with work in progress.

\subsubsection{8. $\quad$ Execution \& Commissioning}

The construction, pre-commissioning, commissioning, and startup (CCSU) stage is a stage where the largest expenditures of money, labor, and time are made in a project's lifecycle. In result, this stage is considered to have the greatest impact on project performance (Almasi 2014; O’Connor et al. 2016). O’Connor and Mock (2019) and Elzarka (2009) found that detailed execution/ commissioning plan, a clear definition of stakeholders agents, a clear scope of work for each agent, and early involvement of agents, and finally logical sequencing of construction events were the best practices for successful execution and commissioning performances. Nevertheless, in many cases, measures needed for effective CCSU are not properly planned or performed, resulting in inefficient start-up and overall operations, as well as cost and schedule overruns. Merrow (2011) conducted a study on oil and gas and mineral megaprojects and concluded that over $70 \%$ of these projects are considered failed projects because they cover only $60 \%$ of the planned production in the first year and over 65\% suffer from longer-term problems. In previous research, a study conducted on 162 projects, found that approximately 50\% of the projects overran the budget for CCSU, and nearly 33 \% exceeded the budget by more than 25\% (O'Connor et al. 2016). The same research showed that $37.5 \%$ of CCSU success-critical variables are not always applied (O'Connor et al. 2016). Therefore, a study conducted by the CII (2016) on best practices recommends implementing Advanced Work Packaging (AWP), as it can significantly improve a project's execution in ways such $25 \%$ improved productivity, $10 \%$ cost savings, improved 
schedule adherence, and improved schedule predictability. The factors studied in the former research were used as a basis for execution and commissioning attributes in this research.

\subsubsection{Change Management \& Project Control}

Change is defined as any event that results in a modification of the original scope, execution time, or cost of work (Hanna and Swanson 2007). A study conducted by Hanna et al. (2004) highlighted the cumulative impact of change orders on labor productivity on mechanical and electrical contractors. The study showed that although change orders are typically compensated in terms of direct cost and time extension, change orders can cause disruptions to site operations that can have indirect cost implications, which can result in productivity loss measured as \%delta. As such, this study quantified the cumulative impact of change orders in terms of six factors: percentage change, change order processing time, overmanning, percentage of project manager's time on the project, percentage of changes initiated by the owner and whether the contractor tracks productivity. An example presented in this study cites the cumulative impact of change orders in the following manner: a $7 \%$ increase in scope can result in a $25 \%$ loss of productivity. The study of Hanna and Iskandar (2018) highlighted that longer change order processing time corresponded to the highest productivity loss (17.75\%) in the data analyzed and showed that change orders often result in increases of 5-10\% in the contract price. Khanzadi et al (2018) have cited previous studies where change orders were the main causes of time and cost overruns (Fallahnejad 2013; Sweis et al. 2008), reworks (Hwang et al. 2014), claims and disputes (Chen and Hsu 2007; Zaneldin 2006). Günhan et al. (2007) showed that the dollar value of change orders can be reduced by taking some preventive measures. Among these measures, are the choice of the right management firm, a clear definition of project scope early in the project, and effective management of precontract activities through performing constructability reviews and value engineering. Shrestha and Shrestha (2019) 
demonstrated that reviewing the specifications before the solicitation of bids, reviewing design drawings, and having an effective change communication system among project teams were important factors to significantly reduce the dollar value of change orders.

A successful progress monitoring plan is another way to tackle performance deficiencies and productivity loss. Orgut et al. (2019) emphasized the major challenges arising from the absence of accurate, coherent, and objective metrics and indicators for project controls, and developed a framework for enhancing the accuracy of tracking systems throughout the project life cycle. Hwang and Liang (2005) explained the significance of constantly tracking productivity information and providing follow-up schedule updates in assessing and predicting cost and schedule performances. Olawale and Sun (2013) developed a Project Control and InhibitingFactors Management Model, which includes a best-practice checklist. Among these practices are ensuring prompt resolutions to RFIs, and allocating enough resources (labor, equipment, etc.) to cope with design changes. In this study, the authors augmented the aforementioned project risk and control attributes, found in the literature.

\subsubsection{Project Competencies and Their Relationship to Project Performance}

Previous research has considered, in many situations, project competencies as a measure of project performance. However, the relationship between the project competencies as leading indicators for project performance improvement has not been well investigated (Fayek 2012). Each of the following paragraphs summarizes the main studies from the previous project competencies literature.

Walsh and Linton (2001) limited their study to core competencies by developing a breakdown that categorized competencies into technical competencies and managerial capabilities. 
Walsh and Linton made a distinction between competencies and capabilities, where competencies were defined as "firm specific technologies and production related skills" (Walsh and Linton 2001, P167), while capabilities were defined as "firm specific business practices, processes and culture" (Walsh and Linton 2001, P167). The framework developed was considered to be a practitioner's guideline to achieve superior performance, but it did not define measurements for performance assessment or relate competencies to performance.

Caupin et al. (2006) have developed and classified comprehensive criteria to evaluate the competency of project management into 20 technical competencies, 15 behavioral competencies, and 11 contextual competencies of project management. Table 3 lists the competence elements for each category. Yet, specific performance measures to evaluate organizational performance or the relation between the competencies to organizational performance were not identified. 
Table 3: Project management competencies elements

\begin{tabular}{|c|c|c|}
\hline Technical Competence & Behavioral Competence & Contextual Competence \\
\hline $\begin{array}{ll}\text { - } & \text { Project management } \\
\text { - } & \text { success } \\
\text { - } & \text { Projestect requirements \& } \\
& \text { objectives } \\
\text { - } & \text { Risk \& opportunity } \\
\text { - } & \text { Quality } \\
\text { - } & \text { Project organization } \\
\text { - } & \text { Teamwork } \\
\text { - } & \text { Problem resolution } \\
\text { - } & \text { Project structures } \\
\text { - } & \text { Scope \& deliverables } \\
\text { - } & \text { Time \& project phases } \\
\text { - } & \text { Resources } \\
\text { - } & \text { Cost \& finance } \\
\text { - } & \text { Procurement \& contract } \\
\text { - } & \text { Changes } \\
\text { - } & \text { Control \& reports } \\
\text { - } & \text { Information \& } \\
\text { - } & \text { documentation } \\
\text { - } & \text { Sommunication } \\
\text { Start-up }\end{array}$ & $\begin{array}{ll}\text { - } & \text { Leadership } \\
\text { - } & \text { Engagement \& } \\
\text { - } & \text { } \text { Selivation } \\
\text { - } & \text { Assertiveness } \\
\text { - } & \text { Relaxation } \\
\text { - } & \text { Openness } \\
\text { - } & \text { Creativity } \\
\text { - Results orientation } \\
\text { - } & \text { Efficiency } \\
\text { - } & \text { Consultation } \\
\text { - Negotiation } \\
\text { - } & \text { Conflict \& crisis } \\
\text { - } & \text { Reliability } \\
\text { - } & \text { Values appreciation } \\
\text { - } & \text { Ethics }\end{array}$ & $\begin{array}{ll}\text { - } & \text { Project orientation } \\
\text { - } & \text { Program orientation } \\
\text { - } & \text { Portfolio orientation } \\
\text { - } & \text { Project programmed \& } \\
& \text { portfolio implementation } \\
\text { - } & \text { Permanent organization } \\
\text { - } & \text { Business } \\
\text { - } & \text { Systems, products \& } \\
& \text { technology } \\
\text { - } & \text { Personnel management } \\
\text { - } & \text { Health, security, safety \& } \\
& \text { environment } \\
\text { - } & \text { Finance } \\
\text { - } & \text { Legal }\end{array}$ \\
\hline
\end{tabular}

Alroomi et al. (2011) proposed an estimating core-competency framework and methodology to prioritize 23 cost estimators' behavioral competencies classified into skills, knowledge, and personal attributes. The framework was developed to prioritize competencies based on the combined effects of the level of importance of each competency and its associated gap between the ideal and actual level of competency. The factor analysis method was then used to group the 23 estimating behavioral competencies into seven factor groups. The limitation of this study is that did not consider other project-competencies that occur throughout the main project phases and did not relate competencies to performance. 
Georgy and Chang (2012) presented a study conducted to investigate how project characteristics impact engineering performance in measurable terms. The project characteristics are classified into six project-specific attributes, three owner attributes, three designer attributes, two schedule attributes, five information inputs, three levels of automation and, three change managements. Project performance included ten performance metrics spanning three project phases: detailed design, fabrication \& construction, and start-up \& commissioning. In this context, utility functions and analytical-hierarchy process (AHP) are integrated to develop an instrument for measuring engineering performance, while Fuzzy Neural Networks (FNNs) are used to develop a connectionist structure that relates engineering performance to its influential project characteristics.

Tabish and Jha (2012) studied the impact of success traits on project success. In their study, success traits were defined as second-order constructs composed of two latent constructs, human factors, and management actions. Structure equation modeling (SEM) was used for this study to investigate the impact of these success traits on the iron triangle of project success (schedule, cost, and quality compliances). The analysis suggested that there is a very strong path between the success traits and project success, with a coefficient of 0.72 . However, this study did not capture the various competency factors that occur throughout the main project phases, and further, it identified only a few performance measures.

Chen (2013) showed how probabilistic models can be applied to identify early warning signs for capital projects in distress. The results of this study support the hypotheses that scope, quality, team, communication, risk, change, and innovation in the initiation and planning phases of capital projects have a potential discriminatory power for differentiating between distressed and non-distressed projects at completion. The findings also indicated that when these variables 
perform well in a project's initiation and planning phases, the project is more likely to meet its budget, schedule, and profitability goals. However, this study only identified competency factors in the initiation and planning phases, and further, it identified only a few performance measures. Furthermore, the study applied an overly simple, and therefore generalizing, way to differentiate between healthy and distressed projects, which followed that a project is healthy $(=1)$, when profitability $=1$, cost $=1$, and time $=1$ exist; otherwise, it is distressed $(=0)$.

A 10-10 performance program was developed by CII (2013). This research distinguished between project performance and project competencies, the latter of which were considered as leading indicators for project performance that are obtained throughout the project's different phases. CII research identified ten sets of inputs (i.e., project competencies) as leading indicators to evaluate project performance. As for outputs, ten outcome measures (i.e., lagging project KPIs) are used to determine if the project is proceeding as planned or not. The input and output metrics are listed in Table 4. Although this study acknowledged the relationship between project competencies and performance, yet it did not quantify the effect of project competencies improvement or lack off on project performance.

Table 4: 10-10 Program input-output metrics

\begin{tabular}{|l|l|}
\hline \multicolumn{1}{|c|}{ Input Metrics } & \multicolumn{1}{c|}{ Output Metrics } \\
\hline 1. Planning & 1. Total project cost/capacity \\
2. Organizing & 2. Total project schedule/capacity \\
3. Leading & 3. Phase cost/capacity \\
4. Controlling & 4. Phase schedule/capacity \\
5. Design efficiency & 5. Phase cost growth \\
6. Human resources & 6. Phase schedule growth \\
7. Quality & 7. Capacity efficiency \\
8. Sustainability & 8. FTE/Total project cost \\
9. Partnering and supply chain & 9. FTE/cost (includes complexity) \\
10. Safety & 10. Phase cost/Phase schedule \\
\hline
\end{tabular}


Omar and Fayek (2014) proposed a framework and methodology for measuring project competencies and performance. The proposed framework categorized project competencies into technical and behavioral project competencies. Technical competencies are classified as either internal or external technical competencies. Internal technical competencies are further divided into: 1) practices that enhance construction projects’ performance (e.g., contract administration practices, financial auditing practices, innovation practices, and workforce improvement practices) or management practices (e.g. scope, change, and risk management practices) that assist in identifying project participants' roles and responsibilities and enhancing the execution of work, and 2) operational competencies, including the application of new technologies (e.g., building information modeling), that contribute to more efficient use of resources. External technical competencies include those that relate to how a construction project connects to stakeholders outside of the construction organization; for example, the communication with stakeholders' competency focuses on the ability of construction project employees to communicate with different construction market stakeholders. The other major group is behavioral competencies are, which are classified as knowledge, skills, or personal attributes. For example, workers' decisionmaking competency is a knowledge-related competency that contributes to the execution of work in construction projects. Learning competency is a personal attribute, a type of behavioral competency, that positively enhances productivity within construction projects. Finally, leadership competency is a personal skill that enhances the overall performance of work in construction projects. Figure 7 shows the proposed project competencies hierarchy. Several categories of performance indicators are identified in this research that were classified into financial, technical, and market performance indicators. Error! Reference source not found. provides some of the categories and lists performance indicators within each category. However, the framework 
developed was conceptual and did not consider validation. This research also did not consider the relationships between construction project competencies and performance indicators and did not develop a model to predict the project performance from the competencies.

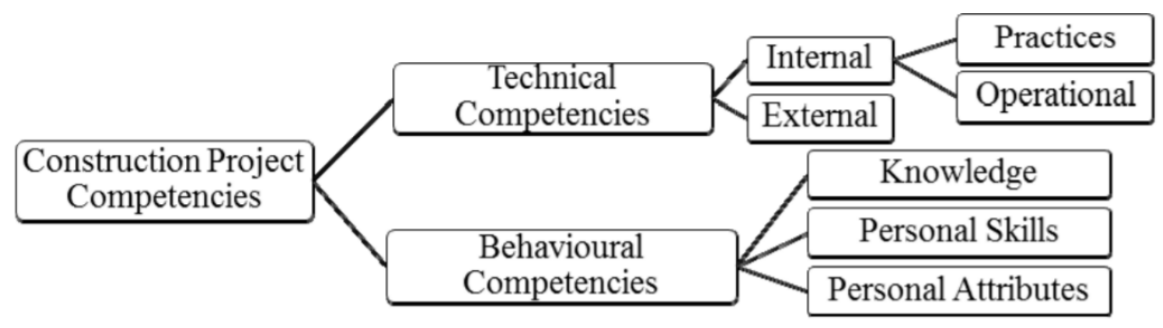

Figure 7: Proposed construction project competencies hierarchy

Table 5: Sample performance indicators for construction projects

\begin{tabular}{|l|l|}
\hline Performance Indicator Category & Performance Indicator \\
\hline Financial Performance Indicators & Indicators Growth Rate \\
Technical Performance Indicators & Reported Safety Incidents \\
Market Performance Indicators & Stakeholders' Satisfaction \\
\hline
\end{tabular}

S. Anantatmula (2015) used the interpretive structural modeling (ISM) methodology to develop a model to determine the relations among influencing factors (project team, policies and procedures, and project goal definitions) and analyzed the underlying interactions that influence project performance (participation in decision making, management support, and project mission).

Figure 8 is an illustration of the proposed project enhancement model. This study did not quantify the effect of project competencies on project performance and considered only a qualitative assessment. 


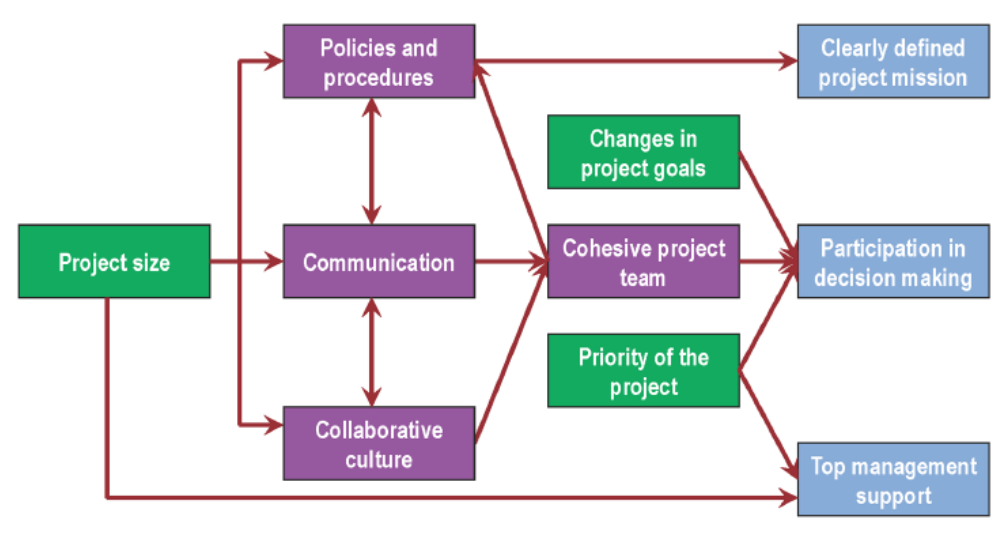

Figure 8: Project Performance Enhancement Model

Luo et al. (2017) analyzed, for the first time, how the complexity of a project impacts project success. First, the complexity of the project is hypothesized to have a negative relationship with the success of the project. Project complexity was measured as information, task, technological, organizational, environmental, and goal complexities by correlation and factor analyses. While project success was measured as time, cost, quality, health and safety, environmental performance, participants' satisfaction, user satisfaction, and commercial value. Finally, the hypothesis and effect of various complexities on project performance were evaluated using the structural equation modeling technique. The findings of this study support the hypothesis that the complexity and success of intricate construction projects are negatively related to each other. Furthermore, information complexity and goal complexity have major negative effects on project success.

Karimi et al. (2018) contributed to the existing literature by quantitatively modeling and elucidating the influence of skilled labor availability on construction projects' cost performance. The primary analysis showed that projects that experienced craft shortages had significantly higher growth in cost overruns compared with projects that did not. These processes include the effects of skilled labor shortage on increasing craft hourly wages and decreasing the quality performance. 
In addition, the impact of time overruns on cost overruns was examined and revealed to be significant as a part of the influence of skilled labor shortages on cost performance through schedule performance. The impact of skilled labor shortages on time overruns was also studied.

Iskandar et al. (2019) developed a mathematical model to quantify and benchmark healthcare project performance in two ways: the PPI and the Team Collaboration and Integration Score (TC\&I). This study mainly aimed to provide a unified quantitative score of performance of healthcare projects using a wide range of metrics as input variables using the following equation.

PPI Score $=0.7966-0.003042 *$ \# of RFIS / \$ Million $+0.0001141 *$ construction speed $0.05956 *$ schedule growth $-0.005584 *$ deficiency issues / \$ Million - 0.06077* percentage change orders due to design issues $-0.1521 *$ percentage of project changes $+0.0001624 *$ delivery speed - 0.06609* cost growth-0.003522* \# of resubmittals / \$Million $-0.005232 *$ rework

This PPI can be proactively used as a standardized productivity tracking metric within a company to assess whether their improvement efforts are effective. This study also offers a unified score for team collaboration and integration using sixty-eight factors spanning seven categories. It is worth mentioning that both unified scores the PPI and TC\&I scores are correlated with the overhead and profit levels achieved by the projects. A statistical investigation of the relationship between TC\&I and PPI showed there is a positive correlation between them of coefficient 0.3: As the level of collaboration/integration increases, the overall project performance increases.

\subsection{Summary Table of Previous Research Studies}

Previous research synopsis identified contributions and limitations in modeling project competencies/performances and their relationship to each other. A summary outlining the main 
contributions of previous research and limitations of each study, as discussed earlier, is presented in Table 6 (Listed by First Author).

Table 6: Summary of previous research in project competencies/performances and their relationships 


\begin{tabular}{|c|c|c|c|c|c|c|c|c|c|c|c|c|c|c|c|c|}
\hline & 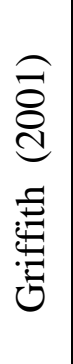 & 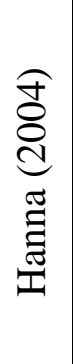 & 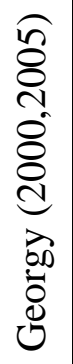 & 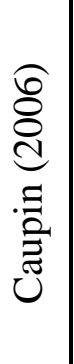 & 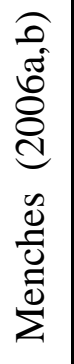 & 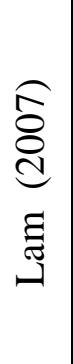 & 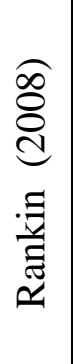 & 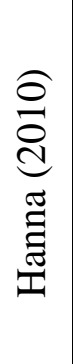 & 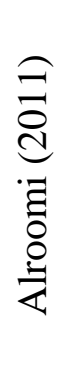 & 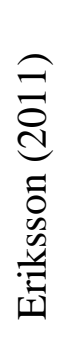 & 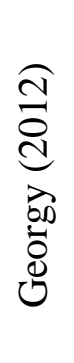 & 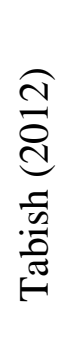 & 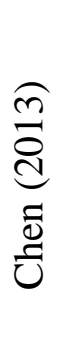 & 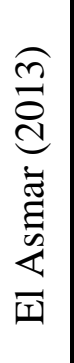 & 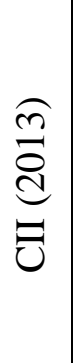 & 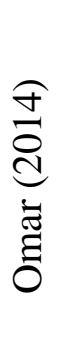 \\
\hline \multicolumn{17}{|l|}{ Project Performance } \\
\hline Cost & & & $*$ & & $*$ & $*$ & $*$ & $*$ & & $*$ & $*$ & $*$ & $*$ & $*$ & $*$ & $*$ \\
\hline Productivity & & $*$ & $*$ & & $*$ & & $*$ & $*$ & & & $*$ & & & $*$ & & \\
\hline Schedule & & & $*$ & & $*$ & $*$ & $*$ & & & $*$ & $*$ & $*$ & $*$ & $*$ & $*$ & \\
\hline Safety & & & & & & & $*$ & $*$ & & $*$ & & $*$ & & $*$ & & $*$ \\
\hline Quality & & & & & & $*$ & $*$ & $*$ & & & & . & & $*$ & & \\
\hline Communication & & & & & $*$ & & & $*$ & & & & & & $*$ & & \\
\hline Integrated framework for performance measures & & & $*$ & & $*$ & $*$ & & $*$ & & & $*$ & $*$ & $*$ & & & \\
\hline Derived weight based on data collected & & & $*$ & & & $*$ & & & & & $*$ & $*$ & & & & \\
\hline \multicolumn{17}{|l|}{ Project Competency } \\
\hline Alignment \& Team Integration & $*$ & & & $*$ & & & & & $*$ & & $*$ & * & $*$ & & $*$ & $*$ \\
\hline Planning \& Design & & $*$ & $*$ & & $*$ & & $*$ & $*$ & $*$ & & $*$ & & & & $*$ & \\
\hline Procurement Management & & & & $*$ & & & $*$ & & & $*$ & & & & & $*$ & \\
\hline Contract \& Risk Management & & & & $*$ & & & & & & & & & $*$ & & & $*$ \\
\hline Health, Safety, \& Environment & & & & $*$ & & & & & & & & & & & $*$ & \\
\hline Quality Management & & & & $*$ & & & & & & & & & $*$ & & $*$ & \\
\hline Labor Productivity & & $*$ & & & & & & & $*$ & & & $*$ & & & $*$ & $*$ \\
\hline Execution \& Commissioning & & & & & & & & & & & & & & & & $*$ \\
\hline Change Management \& Project Control & & $*$ & & $*$ & & & & & & & $*$ & & $*$ & & $*$ & $*$ \\
\hline Integrated framework for competency measures & & & $*$ & & & & & & $*$ & & $*$ & $*$ & & & & \\
\hline Derived weight based on data collected & & & $*$ & & & & & & $*$ & & $*$ & $*$ & & & & \\
\hline \multicolumn{17}{|c|}{ Relationship between Project Competency and Project Performance } \\
\hline Quantify relationship between competency \& performance & & & $*$ & & $*$ & & & $*$ & & & $*$ & * & $*$ & & & \\
\hline
\end{tabular}




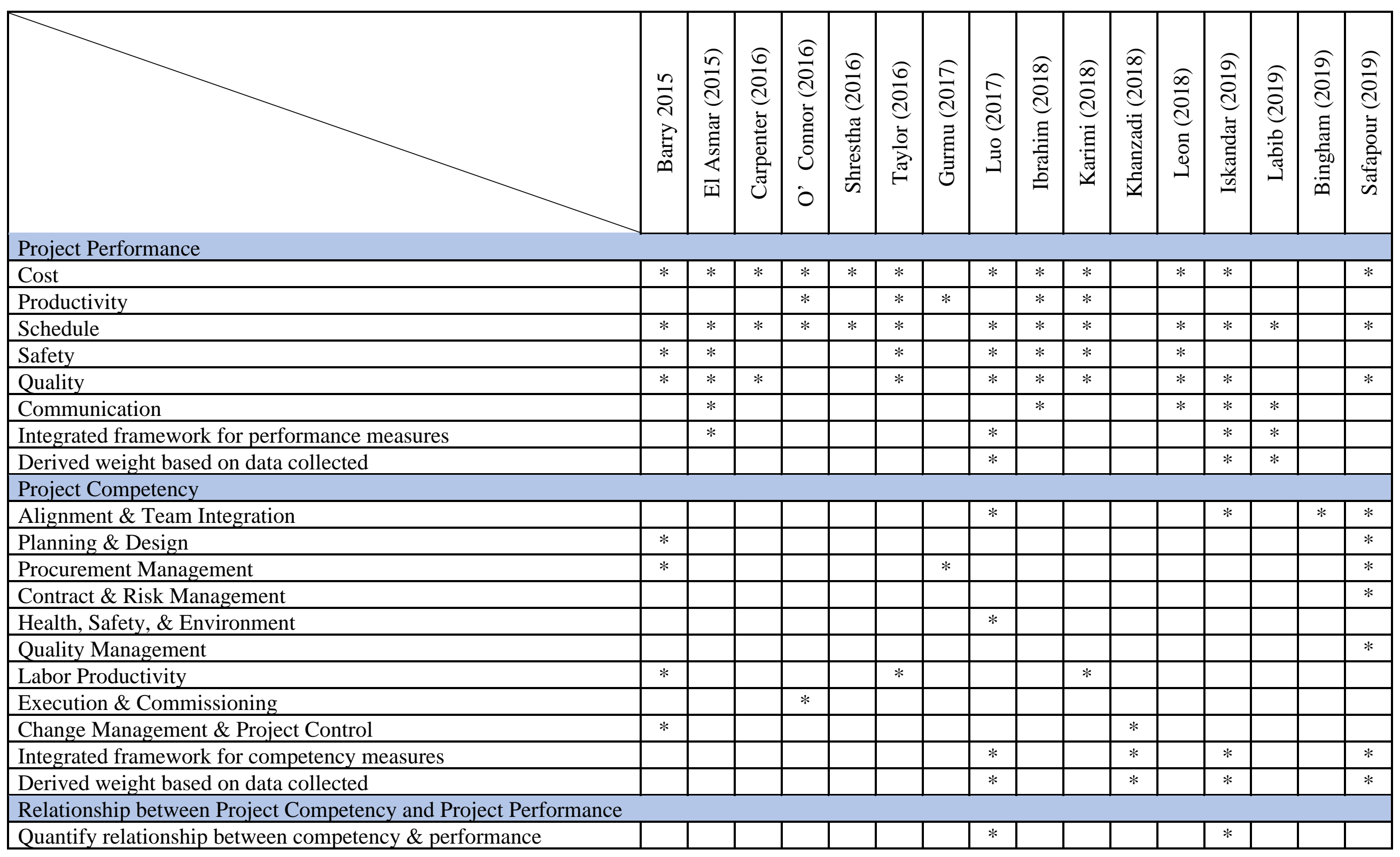




\subsection{Literature Gap}

The limitations of previous studies can be categorized into five principal limitations: 1) few studies conducted a comprehensive assessment and evaluation of the overall performance in different performance areas; 2) none of the studies captured the different categories of project competencies that occur among and across project phases; 3) none of the studies did a comprehensive assessment and evaluation of the various competencies in different competency categories; 4) the subjective nature of the derived weights of the performance/competency metrics in previous studies, as they were based on experts' opinion; 5) limited investigation of the relationship between the project competencies as leading indicators for project performance improvement and quantifying the relative impact of the different project competencies on project performance.

Two integrated mathematical frameworks are used as a robust, accurate, and data-driven weighting technique to achieve the objectives of this paper. First, the paper aims to create a comprehensive performance score by combining the key performance metrics (eight project performance metrics, spanning six performance areas) into one unique cumulative score, named the Project Performance Assessment (PPA) score, which can be used to compare the performance of different projects. Second, this study quantitatively assesses the relative impacts (weights) of the competency factors within its category and uses these factors to compute a competency category-specific score. Finally, the study evaluates the relative impact of the nine different competency categories to the PPA score and provides one unique comprehensive score of the competency categories, named the Competency Category Assessment (CCA) score. The different project competencies identified in this study occur among and across different project phases, as 
recommended by many previous studies (El- Sayegh 2008; Ling et al. 2009; Oke and IdiagbonOke 2010).

The proposed methodology in chapter 1 will allow construction practitioners to proactively evaluate their project's competencies that occur among and across different project phases and enable them to predict the project performance. Thus, it can help them preemptively highlight leverage points of improvement and make comprehensive recommendations to advance project performance accordingly.

\subsection{Summary and Conclusions}

This chapter presented a literature review of previous research in the areas of project competencies, the relationship between project competencies and project performance, and project performance measures, respectively. The limitations of previous research were identified to enhance the existing body of knowledge in project competencies and project performance evaluation and modeling. In response to these limitations, this current research proposes a comprehensive framework to assess the level of competency in terms of the specific category and overall competency level for construction projects, to quantify the overall project performance, and to analyze the relationship between the project competencies as leading indicators of project performance improvement. 


\section{Chapter 3 Data Overview}

The previous chapters introduced this study and its motivation, reviewed previous literature and identified its limitations, and established the research objectives. This chapter will present the final variables used in this study, which include the development of a comprehensive list of eight performance metrics over six holistic areas, and a list of 217 competency factors across nine categories. In addition, this chapter will present a descriptive overview of the studied project characteristics.

\subsection{Identify Research Variables}

A panel of industry expert professionals was established at the beginning of this research. The panel had a balanced owner-to-contractor ratio to provide broad views. The authors then conducted roundtable meetings and in-depth interviews with the collaborating industry members throughout the research project. Since one objective is to provide practical processes to identify the critical competency factors that lead to project success, the industry experts ensured that the findings are an actionable roadmap for contractors to adopt. This technique has been used by several research teams, where industry panels provided industry-related insights (Goodrum et al. 2011; O’Connor and Mock 2019).

\subsubsection{Performance Metrics Assessment Selection Criteria}

As such, the research team developed a project-based survey that was brainstormed through several meetings and workshops with the industry experts, to collect extensive data from both high performing and poor performing projects. The survey was divided into two sections, quantitative and qualitative. The quantitative sections included questions about post-lagging project 
performance metrics such as cost, productivity, schedule, safety, quality, and communication. To ensure that performance metrics provided by participants were as standardized as possible among different projects, this survey adopted some of the key performance metrics definitions used in Hanna et al. (1999a, b) and Hanna’s (2016) research to benchmark project's performance. Figure 9 shows a summary breakdown of the performance areas and metrics used in this research. The following sections show the equations and definitions that were included in the quantitative part to unify the basis of comparisons among participants.

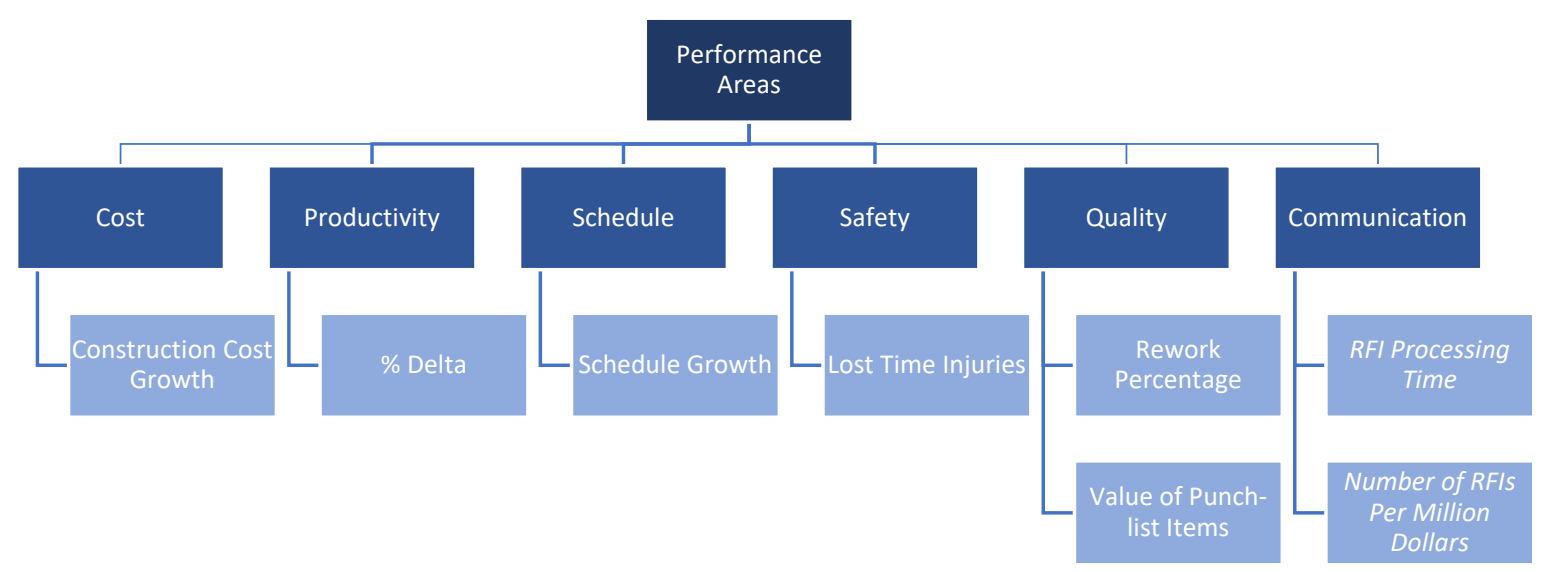

Figure 9: Project performance metrics examined

\subsubsection{Cost Performance}

Cost is one of the important pillars of the "iron triangle" of construction project management. One performance metric was used to assess cost performance, which is the construction cost growth. Construction cost growth is measured in percentage terms by comparing the final construction costs to the original estimated construction costs (Hanna 2016). This metric can be either positive or negative depending on if a project's cost increased or decreased. Construction cost growth is defined using the following equation: 
Construction Cost Growth $=$

$\frac{\text { Actual Construction cost growth }- \text { Initial Construction cost growth }}{\text { Initial Construction cost growth }} \times 100 \%$

\subsubsection{Productivity Performance}

Construction is a labor-intensive industry. Therefore, the financial success of any construction project requires outstanding labor management and high productivity control. Thus, percent delta was used in this study as an indicator by which to assess productivity performance. Percent delta measures the lost productivity hours as a percent of the total actual hours utilized by the time of project completion. This metric can be either positive or negative depending on if the project's labor hours increased or decreased throughout the project. \%Delta is defined using the following equation, as defined by Hanna et al. (1999a, b):

Percent delta $=\frac{\text { Actual direct hours-(Initial estimated hours }+ \text { Approved Changes })}{\text { Actual direct hours }} \times 100 \%$

\subsubsection{Schedule Performance}

Schedule performance is often cited as a major side of the "iron triangle" of performance metrics along with cost and quality. In this research, schedule performance is measured using construction schedule growth. Construction schedule growth is measured in percentage terms by comparing the final construction schedule to the original estimated construction schedule (Hanna 2016). This metric can be either positive or negative depending on if the project's duration 
increased or decreased from its expected duration. Construction schedule growth is defined using the following equation:

Construction schedule growth $=$ Actual Construction schedule growth - Initial Construction schedule growth Initial Construction schedule growth

\subsubsection{Safety Performance}

The construction industry is characterized by the Occupational Safety and Health Administration (OSHA) as a high-risk industry. Consequently, the safety performance of contractors has become increasingly prioritized and thus is one of the important variables influencing companies’ decisions to award contracts. This thesis examined lost-time injuries (LTI) as an indicator by which to gauge the safety performance. LTI is defined as injuries and/or illnesses resulting in lost workdays or restricted work activity (Hanna 2016). LTI is measured quantitatively, by normalizing them using the project's total cost.

\subsubsection{Quality Performance}

In the construction industry, one of the pillars of every project delivery process is the quality management control of the materials and services provided. Hence, quality performance is cited as the third major side of the "iron triangle" of performance metrics with cost and time. Both qualitative and quantitative metrics were used to assess quality performance comprehensively. There are two quality performance metrics evaluated in this thesis, which are: 1) rework, and 2) punch list items per million dollars. Rework is measured in percentage terms by comparing the 
total direct cost to the actual construction cost (Hanna 2016). The rework percentage is defined using the following equation:

$$
\text { Rework }=\frac{\text { Total Direct cost of field Rework }}{\text { Actual Costruction Cost }} * 100
$$

The second indicative factor of quality is punch-list items, which are incomplete or unsatisfactory items that remain after the substantial completion of a project. The value of the punch list items is assessed qualitatively, where $1=$ very low, 2 = low, $3=$ moderate, $4=$ high, and 5 = very high (Hanna 2016).

\subsubsection{Communication Performance}

Successful construction projects rely heavily on effective information exchange among the members of multidisciplinary teams on the project. Therefore, good communication is highly correlated with project success and poor communication problems can result in significantly poorer project performance (Iskandar et al. 2019). This thesis used the following metrics to assess communication performance: 1) RFI processing time; 2) the number of RFIs per million dollars.

The number of RFIs per million dollars refers to the number of RFIs submitted from the construction team to the design team. The number of RFIs per million dollars is measured quantitatively by normalizing them using the project's total costs (Hanna 2016). This measurement allows for the comparison of different-sized projects to one another. On the other hand, the average RFI processing time is defined as the duration between the submittal of an RFI and the response by the appropriate party/closure (Hanna 2016). This is measured qualitatively on an ordinal scale 
ranging from 1 to 5 , where: 1 = (1-7 days); 2 = (8-14 days); $3=(15-21$ days $) ; 4=(22-28$ days $)$; and $5=(>28$ days $)$.

\subsubsection{Ranges of the performance metrics}

The range of values for the performance metrics for the collected projects is summarized in Table 7

Table 7: Range of values for performance metrics for the studied projects

\begin{tabular}{|c|c|c|c|}
\hline Performance Area & Performance Metrics & $\begin{array}{l}\text { Measurement } \\
\text { Type }\end{array}$ & $\begin{array}{c}\text { Range in } \\
\text { dataset }\end{array}$ \\
\hline Cost performance & Construction Cost Growth & $\begin{array}{l}\text { Numerical } \\
\text { (percent) }\end{array}$ & $-0.91 \rightarrow+2.86$ \\
\hline $\begin{array}{l}\text { Productivity } \\
\text { performance }\end{array}$ & Percent Delta & $\begin{array}{l}\text { Numerical } \\
\text { (percent) }\end{array}$ & $-9.55 \rightarrow+1.85$ \\
\hline $\begin{array}{l}\text { Schedule } \\
\text { performance }\end{array}$ & Construction Schedule Growth & $\begin{array}{l}\text { Numerical } \\
\text { (percent) }\end{array}$ & $-1 \rightarrow+1.75$ \\
\hline Safety performance & $\begin{array}{l}\text { Lost Time Injuries per Million } \\
\text { Dollars }\end{array}$ & $\begin{array}{l}\text { Numerical } \\
\text { (Ratio) }\end{array}$ & $0 \rightarrow 2.75$ \\
\hline Safety performance & Rework percentage & $\begin{array}{l}\text { Numerical } \\
\text { (percent) }\end{array}$ & $0.0018 \rightarrow 1$ \\
\hline $\begin{array}{l}\text { Quality } \\
\text { performance }\end{array}$ & Value of Punch-list Items & $\begin{array}{c}\text { Ordinal }(1,2,3,4 \\
\text { or } 5)\end{array}$ & $1 \rightarrow 5$ \\
\hline $\begin{array}{l}\text { Communication } \\
\text { performance }\end{array}$ & $\begin{array}{l}\text { Number of RFIs per Million } \\
\text { Dollars }\end{array}$ & $\begin{array}{l}\text { Numerical } \\
\text { (Ratio) }\end{array}$ & $0.092 \rightarrow 1259.4$ \\
\hline $\begin{array}{l}\text { Communication } \\
\text { performance }\end{array}$ & RFI Processing Time & Ordinal (weeks) & $1 \rightarrow 5$ \\
\hline
\end{tabular}

\subsubsection{Project Competency Assessment Selection Criteria}

The second section in the survey, the qualitative section, was brainstormed through several meetings and workshops with the industry experts. The authors (1) identified 217 comprehensive competency factors, and (2) grouped the competency factors into nine categories using the Kawakita Jiro (KJ) method. The KJ method, a bottom-up sorting process, was developed by Kawakita Jiro in 1953, and it is used to organize data into useful categories and transform the data 
into information (Cheng and Leu 2011). The KJ method starts with (1) gathering data, (2) determining theme, (3) sorting data into groups according to related ideas or topics, and (4) creating a header for each group (Plain 2007). Figure 10 shows a summary of the nine competency categories and the following tables show the list of the competency factors in their corresponding categories.

According to PMI (2008), “project management is the application of knowledge, skills, tools, and techniques to a broad range of activities in order to meet the requirements of a particular project.” Hence, the competency factors selection process attempts to capture the main events and interactions that occur throughout the main project phases (pre-construction, construction, postconstruction). It is important to consider that some categories occur in more than one stage.

Notably, just because a category has more factors in it does not mean that it is more important or a more likely predictor of project performance than a category with fewer factors. Alternatively, the number of factors per category is an indicator of the type of category. A category with more factors is indicative of a higher level of detail and consideration put in place by the project team before construction. Additionally, the number of factors per category is indicative of that category's pertinence to preconstruction activities. For example, "planning” obviously is highly correlated with preconstruction activities, and as such has a higher number of associated factors.

The survey questions about the identified 217 competency factors used a binary scale; if a project made use of a competency factor, the participant assigned a 1 to that question; if not, a 0. Table 8-16 summarizes the competency factors in their assigned categories. 


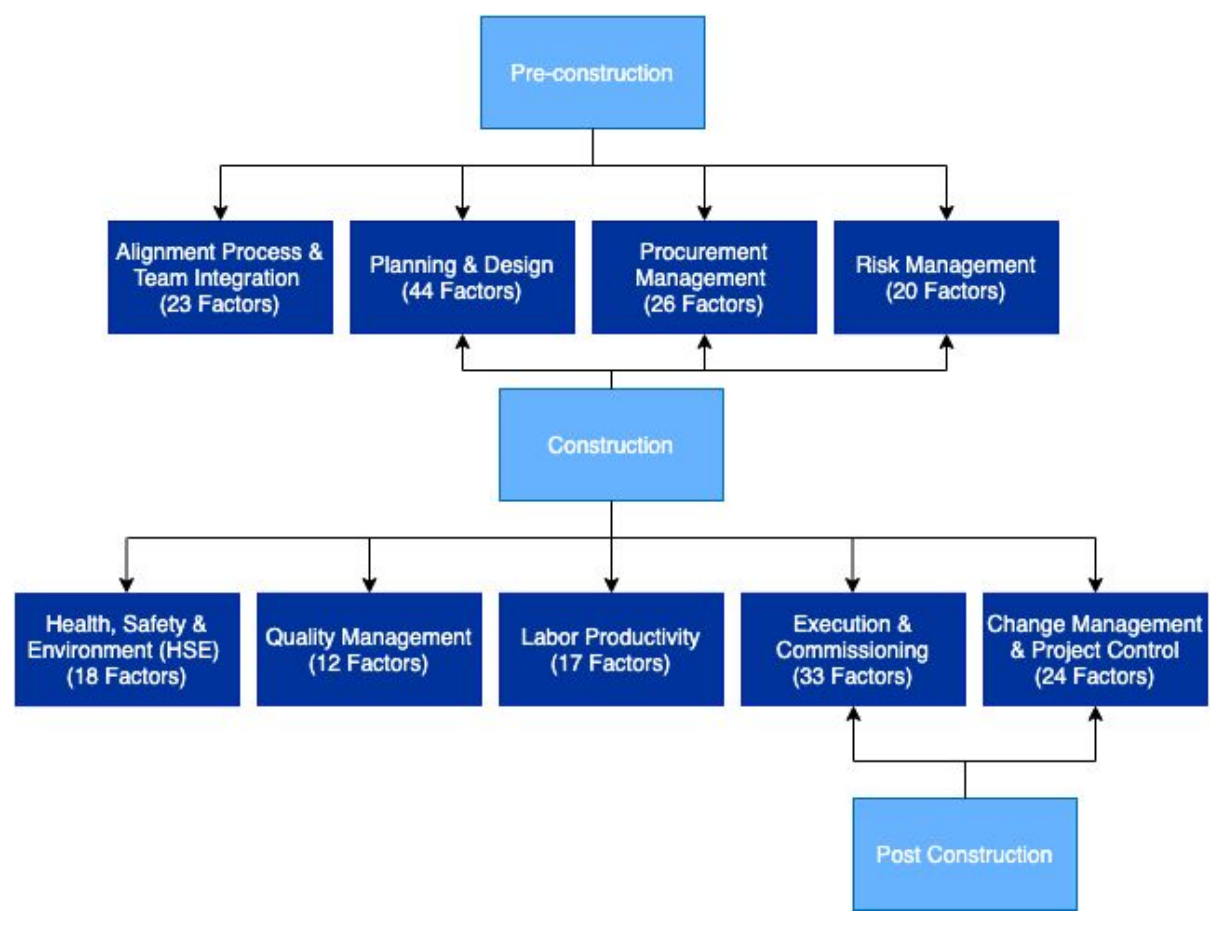

Figure 10: Summary of the competency categories across the project life cycle

Table 8: Alignment Process \& Team Integration (AL\&T)

\begin{tabular}{|l|l|}
\hline Code & Factor \\
\hline AL\&T 1 & Project objectives defined by team members \\
\hline AL\&T 2 & Project drivers (cost/schedule) been agreed on by the team \\
\hline AL\&T 3 & Project objectives are well documented \\
\hline AL\&T 4 & Project objectives communicated by team members \\
\hline AL\&T 5 & Engineering Personnel are included in project team from the preconstruction phase. \\
\hline AL\&T 6 & Procurement Personnel are included in project team from the preconstruction phase. \\
\hline AL\&T 7 & Fabrication Personnel are included in project team from the preconstruction phase. \\
\hline AL\&T 8 & Construction Personnel are included in project team from the preconstruction phase. \\
\hline AL\&T 9 & Subcontractors are included in project team \\
\hline AL\&T 10 & Responsible, Accountable, Consulted and Informed (RACI) matrix was developed for the project \\
\hline AL\&T 11 & Project team roles and responsibilities are well defined \\
\hline AL\&T 12 & Roles and responsibilities are well communicated to the project team \\
\hline AL\&T 13 & All key leadership positions of the project team are filled \\
\hline AL\&T 14 & The mission is entrusted to experienced leaders \\
\hline AL\&T 15 & There is defined support system to allocate resources \\
\hline AL\&T 16 & Frequency of project team meetings are well defined \\
\hline AL\&T 17 & Frequency of progress reports are well defined \\
\hline AL\&T 18 & Project team formed in a timely manner \\
\hline AL\&T 19 & Project team has all required skills \\
\hline
\end{tabular}




\begin{tabular}{|l|l|}
\hline AL\&T 20 & Project team has the expertise to take on the project \\
\hline AL\&T 21 & Project team is well trained for project-specific procedures \\
\hline AL\&T 22 & There is cultural diversity in the project team \\
\hline AL\&T 23 & A system of oversight and governance been established for the project \\
\hline
\end{tabular}

\section{Table 9: Planning \& Design (PL\&D)}

\begin{tabular}{|c|c|}
\hline Code & Factor \\
\hline PL\&D 1 & Project budget has been defined \\
\hline PL\&D 2 & A master schedule is made \\
\hline PL\&D 3 & Master schedule considers subcontractor schedules \\
\hline PL\&D 4 & Master schedule considers owner furnished items schedules \\
\hline PL\&D 5 & The master schedule is designed to support program transition to turnover \\
\hline PL\&D 6 & The master schedule includes all tasks and activities \\
\hline PL\&D 7 & Critical path has been identified \\
\hline PL\&D 8 & Near critical path has been identified \\
\hline PL\&D 9 & Master schedule is baselined \\
\hline PL\&D 10 & Total project duration and milestones are well defined \\
\hline PL\&D 11 & Planned activities durations are in line with project conditions \\
\hline PL\&D 12 & The labor productivity rates for major items are in line with recent experiences \\
\hline PL\&D 13 & a comprehensive Work Breakdown Structure (WBS) has been developed \\
\hline PL\&D 14 & The WBS supports commissioning and system turnover \\
\hline PL\&D 15 & Requirements of different stakeholders are considered in the planning process \\
\hline PL\&D 16 & A planned resource loading is made to consider buildup, peak, and rundown \\
\hline PL\&D 17 & Labor resource requirements were supported in the project \\
\hline PL\&D 18 & Hold points/handoffs are identified \\
\hline PL\&D 19 & Hold points/handoffs are integrated with the schedule \\
\hline PL\&D 20 & Overmanning considered in the schedule \\
\hline PL\&D 21 & There is enough space available to avoid the stacking of trades for each worker \\
\hline PL\&D 22 & The project logistics plan is incorporated in the schedule \\
\hline PL\&D 23 & The project testing plan is incorporated in the schedule \\
\hline PL\&D 24 & The temporary facilities plan is incorporated in the schedule \\
\hline PL\&D 25 & Resource leveling (especially manpower) is considered in schedule \\
\hline PL\&D 26 & Craft-supervision ratio is identified \\
\hline PL\&D 27 & The s-curve including the cash flow and rate of expenditures are defined \\
\hline PL\&D 28 & All engineering milestones are developed \\
\hline PL\&D 29 & All engineering deliverables in construction packages are defined \\
\hline PL\&D 30 & Standards and specifications are well defined \\
\hline PL\&D 31 & IFC drawings are issued to support the construction activity \\
\hline PL\&D 32 & Schedule for design deliverables compatible with the construction sequence \\
\hline PL\&D 33 & Clash detection checks are implemented \\
\hline PL\&D 34 & There is a well-defined procedure for receiving vendor information \\
\hline PL\&D 35 & Design incorporated in commissioning and startup \\
\hline
\end{tabular}




\begin{tabular}{|l|l|}
\hline PL\&D 36 & The different disciplines of design are well coordinated \\
\hline PL\&D 37 & Engineering responsibilities are well defined \\
\hline PL\&D 38 & Design incorporated operability \\
\hline PL\&D 39 & There is a defined process for responding to RFIs \\
\hline PL\&D 40 & Submittal process is well defined \\
\hline PL\&D 41 & There is a clear process for approval of shop drawings \\
\hline PL\&D 42 & There is a clear process for updating drawings \\
\hline PL\&D 43 & There is a clear process for reporting RFIs impacting schedule and cost \\
\hline PL\&D 44 & There is a defined system of engineering support (incl. planned on-site availability) \\
\hline
\end{tabular}

\section{Table 10: Procurement Management (Proc)}

\begin{tabular}{|c|c|}
\hline Code & Factor \\
\hline Proc 1 & Specialty tools/equipment have been identified and secured \\
\hline Proc 2 & There is a contingency plan (backup, maintenance, etc.) for critical tools and equipment \\
\hline Proc 3 & There is an acquisition management plan for tools and equipment in place \\
\hline Proc 4 & There is a mobilization management plan for tools and equipment in place \\
\hline Proc 5 & There is a placement management plan for tools and equipment in place \\
\hline Proc 6 & There is a storage management plan for tools and equipment in place \\
\hline Proc 7 & There is a movement management plan for tools and equipment in place \\
\hline Proc 8 & There is a control management plan for tools and equipment in place \\
\hline Proc 9 & There is a maintenance management plan for tools and equipment in place \\
\hline Proc 10 & All pieces of equipment are inspected and certified \\
\hline Proc 11 & Roads, access, assembly areas, and foundations are identified and secured before construction \\
\hline Proc 12 & Tools \& consumables are identified and secured before construction \\
\hline Proc 13 & Vendor documents required for construction, commissioning, and startup are managed beforehand \\
\hline Proc 14 & There is a defined plan for storage, staging, and preservation \\
\hline Proc 15 & Ordering plan for materials is secured \\
\hline Proc 16 & Tracking plan for materials is secured \\
\hline Proc 17 & Delivery plan for materials is secured \\
\hline Proc 18 & Storage plan for materials is secured \\
\hline Proc 19 & Transportation plan for materials is secured \\
\hline Proc 20 & Installation plan for materials is secured \\
\hline Proc 21 & Surplus plan for materials is secured \\
\hline Proc 22 & There is a material control system (in terms of security and tracking) in place \\
\hline Proc 23 & All bulky materials needed for start of construction are purchased \& on site \\
\hline Proc 24 & There is a system in place to pre-package material according to individual work packages \\
\hline Proc 25 & There is a procedure in place to report over, short, and damaged (OS\&D) incidents for the materials \\
\hline Proc 26 & There is a field procurement capability on site \\
\hline
\end{tabular}


Table 11: Contract \& Risk Management (CNRM)

\begin{tabular}{|l|l|}
\hline Code & Factor \\
\hline CNRM 1 & $\begin{array}{l}\text { There is a common understanding of all terms and conditions of the contract among the project } \\
\text { team }\end{array}$ \\
\hline CNRM 2 & Notification requirements are communicated among the project team \\
\hline CNRM 3 & The invoice submittal process clearly communicated and understood by the project team \\
\hline CNRM 4 & $\begin{array}{l}\text { The procedures that should be followed in case of changes= orders are communicated among the } \\
\text { project team }\end{array}$ \\
\hline CNRM 5 & The implemented contractual incentives/liquidated damages are clear \\
\hline CNRM 6 & Contractual flow between tiers (flow-down clauses) are consistent \\
\hline CNRM 7 & The project completeness criteria are well-defined \\
\hline CNRM 8 & Scope of prime contractor is well defined \\
\hline CNRM 9 & Legal/contracts team and other technical teams coordinate to draft subcontracts \\
\hline CNRM 10 & Claims management process is well-defined \\
\hline CNRM 11 & Dispute resolution procedures are well-defined early in the project \\
\hline CNRM 12 & The warranty period and the processing of interim payments is clearly written in the contract \\
\hline CNRM 13 & Defined delegation/signing authority are well-known among the project team \\
\hline CNRM 14 & Force majeure (excusable non-compensable) clauses are communicated among the project team \\
\hline CNRM 15 & Having a system to ensure insurance is provided \\
\hline CNRM 16 & Risks impacting the construction phase of the project are well-assessed \\
\hline CNRM 17 & Risk identification and evaluation process are well defined \\
\hline CNRM 18 & Risk analysis and investigation process are well defined \\
\hline CNRM 19 & Risk monitoring and response framework are well defined in terms of cost \\
\hline CNRM 20 & Risk monitoring and response framework are well defined in terms of schedule \\
\hline
\end{tabular}

\section{Table 12: Health, Safety \& Environmental Factors (HSE)}

\begin{tabular}{|l|l|}
\hline Code & Factor \\
\hline HSE 1 & There is an emergency plan in place \\
\hline HSE 2 & There is medical support defined for the project \\
\hline HSE 3 & There is an environmental management plan in place \\
\hline HSE 4 & The project team is aligned on the site-specific safety plan \\
\hline HSE 5 & The site hazard identification (walkthroughs) has been completed \\
\hline HSE 6 & The site security control procedures are in place \\
\hline HSE 7 & The pre-employment checks on workers are adequate (i.e. drug testing, background checks) \\
\hline HSE 8 & There is an integrated HSSE plan in place for simultaneous operations \\
\hline HSE 9 & There is a defined process for communicating changes to the safety plan \\
\hline HSE 10 & All the environmental permits that are necessary for starting construction have been obtained \\
\hline HSE 11 & On-site safety communication (safety signage/safety board locations) is in place \\
\hline HSE 12 & The comprehensive blinding, clearing, lockout-tagout and unit entry plans are in place \\
\hline HSE 13 & All special safety-related training requirements are included in the plan \\
\hline HSE 14 & There is a safety induction/orientation plan in place \\
\hline HSE 15 & The subcontractor's safety plans meet the required standards \\
\hline
\end{tabular}




\begin{tabular}{|l|l|}
\hline HSE 16 & There are planned safety and toolbox meetings \\
\hline HSE 17 & There is a defined work permit process in place \\
\hline HSE 18 & There is a defined safety supervision plan in place \\
\hline
\end{tabular}

\section{Table 13: Quality Management (QM)}

\begin{tabular}{|l|l|}
\hline Code & Factor \\
\hline QM 1 & Quality assurance plan is in place \\
\hline QM 2 & Quality assurance practices have been defined and communicated \\
\hline QM 3 & Quality control plan performed on procured items, fabrication, and construction \\
\hline QM 4 & There are well-defined acceptance criteria for major items \\
\hline QM 5 & Quality control plan communicated among the project team \\
\hline QM 6 & people, tools, systems are in place to perform the quality control plan \\
\hline QM 7 & Quality inspection procedures are well defined \\
\hline QM 8 & Roles and responsibilities are well-defined for quality inspection \\
\hline QM 9 & Inspection and testing plan is in place \\
\hline QM 10 & There is a defined process for third party inspection, including auditing \\
\hline QM 11 & There is a defined process for third party vendor or tech support \\
\hline QM 12 & A clear protocol for cases of non-compliance or corrective action is defined \\
\hline
\end{tabular}

Table 14: Labor Productivity (LP)

\begin{tabular}{|c|c|}
\hline Code & Factor \\
\hline LP 1 & The project team completed a review and analysis of the labor market, including competing projects in the area \\
\hline LP 2 & A plan to secure and retain skilled labor \\
\hline LP 3 & Has a plan in place to minimize craft absenteeism \\
\hline LP 4 & The project team understands the craft labor agreement requirements (including restrictive practices) \\
\hline LP 5 & Billable labor rates have been established \\
\hline LP 6 & A craft evaluation/qualification program is in place \\
\hline LP 7 & A project-specific training program is in place \\
\hline LP 8 & Available number of project staff is secured and retained \\
\hline LP 9 & The procedures for owner approval of hiring and rotating key personnel are agreed upon by the project team \\
\hline LP 10 & On-boarding procedures are in place to on-board new hires \\
\hline LP 11 & Expectations for active employees’ procedures are in place to on-board new hires \\
\hline LP 12 & Progressive discipline procedures are in place to on-board new hires \\
\hline LP 13 & Termination procedures are in place to on-board new hires \\
\hline LP 14 & Promotion procedures in place to on-board new hires \\
\hline LP 15 & Labor demobilization procedures are well defined \\
\hline LP 16 & There is an established system to record the attendance of employees to the site \\
\hline LP 17 & There is a program in place for Short Service Employees (SSE) \\
\hline
\end{tabular}


Table 15: Execution \& Commissioning (E\&C)

\begin{tabular}{|c|c|}
\hline Code & Factor \\
\hline E\&C 1 & There are relevant clauses in the contract to support construction schedules \\
\hline E\&C 2 & There is a defined process for approving subcontractors and vendors \\
\hline E\&C 3 & Construction execution plan is well defined \\
\hline E\&C 4 & Construction execution plan is aligned with the project execution plan \\
\hline E\&C 5 & Construction execution plan has been well-communicated to the project team \\
\hline E\&C 6 & Contractors are familiar with their obligations \\
\hline E\&C 7 & All project teams are familiar with the contractors' obligations \\
\hline E\&C 8 & Mobilization plan is well defined \\
\hline E\&C 9 & There are good communication tools in place \\
\hline E\&C 10 & A coordination plan is well defined between all project parties \\
\hline E\&C 11 & There are adequate vertical transportation (cranes, elevators, etc.) \\
\hline E\&C 12 & There is a plan for people movement: - To and from the site \\
\hline E\&C 13 & There is a plan for people movement: - Within the site \\
\hline E\&C 14 & There is a plan for materials movement: - To and from the site \\
\hline E\&C 15 & There is a plan for materials movement: - Within the site \\
\hline E\&C 16 & There is a plan for large equipment movement: - To and from the site \\
\hline E\&C 17 & There is a plan for large equipment movement: - Within the site \\
\hline E\&C 18 & All necessary utilities are available on site (including electricity, water, drainage, etc.) \\
\hline E\&C 19 & A plan to minimize out-of-sequence work are well defined \\
\hline E\&C 20 & Turnover procedures (from construction to commissioning) are well defined \\
\hline E\&C 21 & Construction aligned with commissioning and operations \\
\hline E\&C 22 & There is a defined system for the transition from area-based to system-based \\
\hline E\&C 23 & The commissioning and startup plan is integrated with the EPC schedule \\
\hline E\&C 24 & There is an engagement timeline of the commissioning group \\
\hline E\&C 25 & A dedicated commissioning leader has been identified and communicated \\
\hline E\&C 26 & $\begin{array}{l}\text { There is a defined Health-Safety-Security-Environment (HSSE) transition plan from construction to } \\
\text { operations }\end{array}$ \\
\hline E\&C 27 & $\begin{array}{l}\text { There is a comprehensive commissioning manual (including all procedures and preservation } \\
\text { requirements) }\end{array}$ \\
\hline E\&C 28 & The provisions for QA/QC turnover documentation are understood by the project team \\
\hline E\&C 29 & There is a mechanical completion checklist in place \\
\hline E\&C 30 & There is a punch list process in place \\
\hline E\&C 31 & The as-built documentation procedure is understood by the project team \\
\hline E\&C 32 & The strategy to manage equipment closure is well-communicated and understood \\
\hline E\&C 33 & Pre-start-up safety review requirements are well-communicated and understood \\
\hline
\end{tabular}


Table 16: Change Management \& Project Control (CMPC)

\begin{tabular}{|c|c|}
\hline Code & Factor \\
\hline CMPC 1 & Change management process is in place \\
\hline CMPC 2 & Identifying enough components of the change management process to start construction \\
\hline CMPC 3 & Identification of change orders process is well-defined \\
\hline CMPC 4 & Evaluation and quantification of change order process are well defined \\
\hline CMPC 5 & Knowing the authorized entity to issue change orders \\
\hline CMPC 6 & Procedure for the implementation of field change orders is well-defined \\
\hline CMPC 7 & Document control of change orders are well defined \\
\hline CMPC 8 & Tracking change orders \\
\hline CMPC 9 & Payment procedures for changes are well defined \\
\hline CMPC 10 & Change orders are reconciled to scope \\
\hline CMPC 11 & Chain of communication of change orders are well defined to stakeholders \\
\hline CMPC 12 & Change management roles and responsibilities are well defined to project parties \\
\hline CMPC 13 & Identifying change management process among project team \\
\hline CMPC 14 & Turnover procedures for change management are well defined \\
\hline CMPC 15 & Having a process to document rework \\
\hline CMPC 16 & Progress tracking plan and systems are in place \\
\hline CMPC 17 & The resources to support project tracking are deployed \\
\hline CMPC 18 & A process for responding to delays is well-defined \\
\hline CMPC 19 & A process to collect, compile and report costs is well-defined \\
\hline CMPC 20 & A process to monitor and control productivity is well-defined \\
\hline CMPC 21 & Updating schedule process is well defined \\
\hline CMPC 22 & Document control system is defined \\
\hline CMPC 23 & A control budget (at the work package level) is in place \\
\hline CMPC 24 & Cost contingency management plan is well defined \\
\hline
\end{tabular}

\subsection{Data Characteristics \& Overview}

After this step, the authors expanded the list of questions, adding sections that gathered data related to project features (e.g., location or size). The authors pilot tested the survey internally through collaborating industry members who used data from projects executed by their companies. Additionally, industry collaborators tested the ease and clarity of the survey to ensure data to be collected aligned with research objectives. A total of 120 projects were obtained. Understanding these characteristics is essential because it helps ensure that the sampled projects are representative of the overall population of projects. 


\subsubsection{Geographic Distribution}

There was no specified geographic preference for the data. Despite this, $89 \%$ of the surveyed projects were in North America, of which 90 projects were in the United States and the remaining 17 projects were in Canada. The rest of the projects were in South America, Africa, Asia, and Europe with percentages of $6 \%, 3 \%, 2 \%$, and 1\%, respectively. Figure 11 shows the geographic distribution of the studied projects.

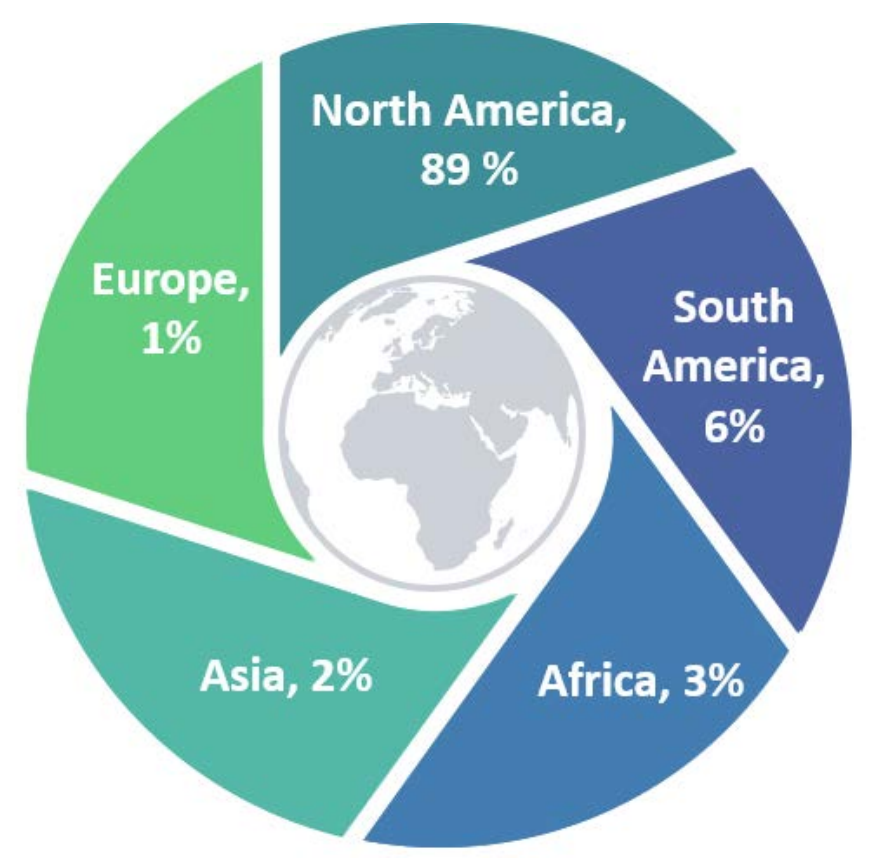

Figure 11: Geographic distribution of studied projects

\subsubsection{Respondent's Project Role}

The respondents were either general contractors/construction managers (GC/CM) (46\%), owners (28\%), subcontractors (24\%), or engineers (2\%). Figure 12 depicts the distribution of the respondent's roles in the studied projects. 


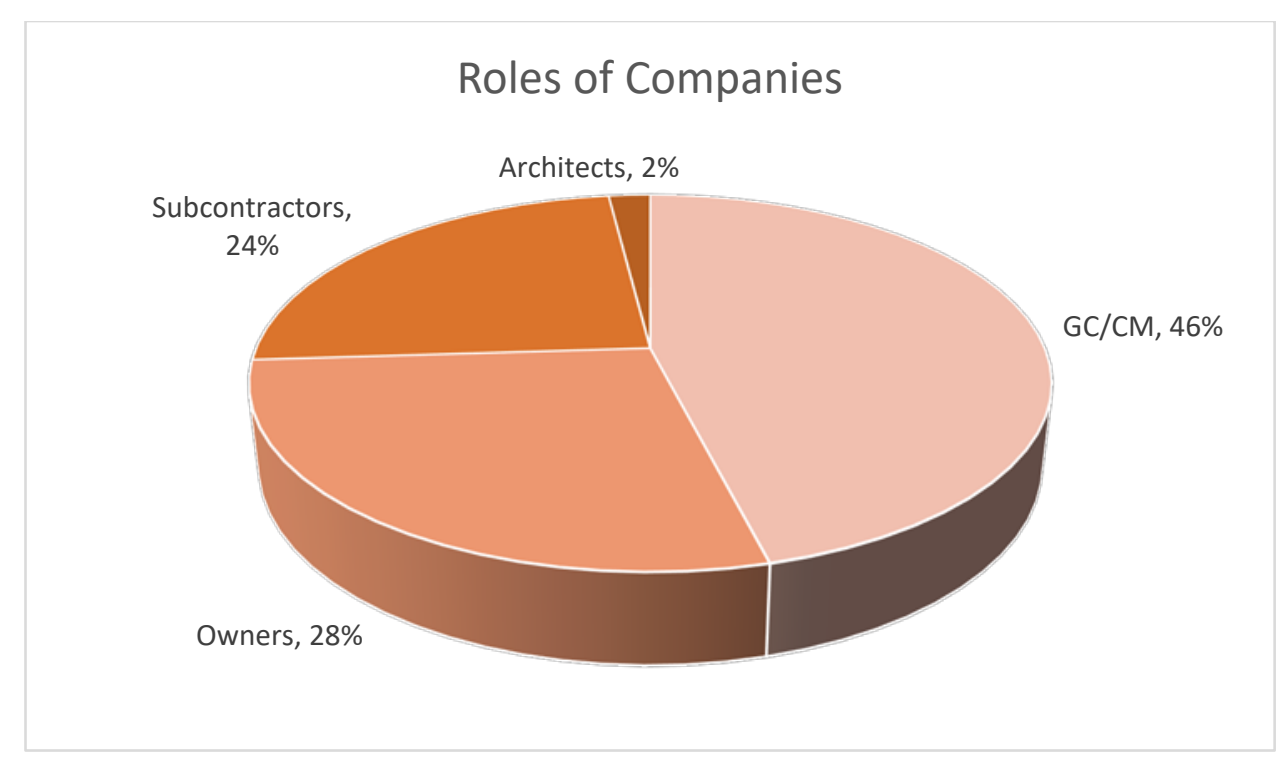

Figure 12: Respondent's roles in studied projects

\subsubsection{Industry Sector}

The datasets include different industry groups, and most of the projects were largely industrial (70.1\%), with buildings, infrastructure, and renovation representing $17.1 \%$, 10.3\%, and 2.6\%, respectively. A full breakdown of industry sectors is depicted in Figure 13. 


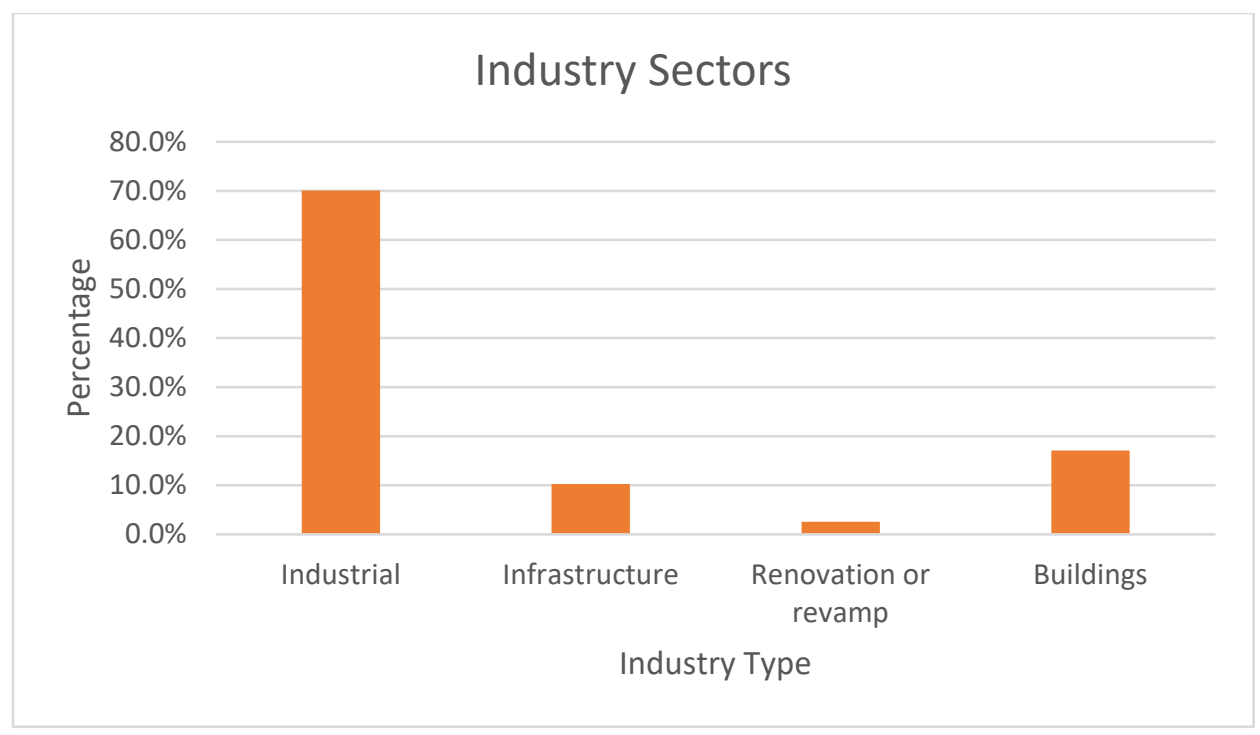

Figure 13: Industry sector of studied projects

\subsubsection{Construction Work Types}

The dataset also includes various construction work types including grassroots/greenfield with $35.3 \%$ of projects. modernization, renovation, and upgrade (31.9\%) were the next most prevalent. Brownfield and addition/expansion were also represented having $21.6 \%$ and $11.2 \%$, respectively. A full breakdown of the work types is depicted in Figure 14. 


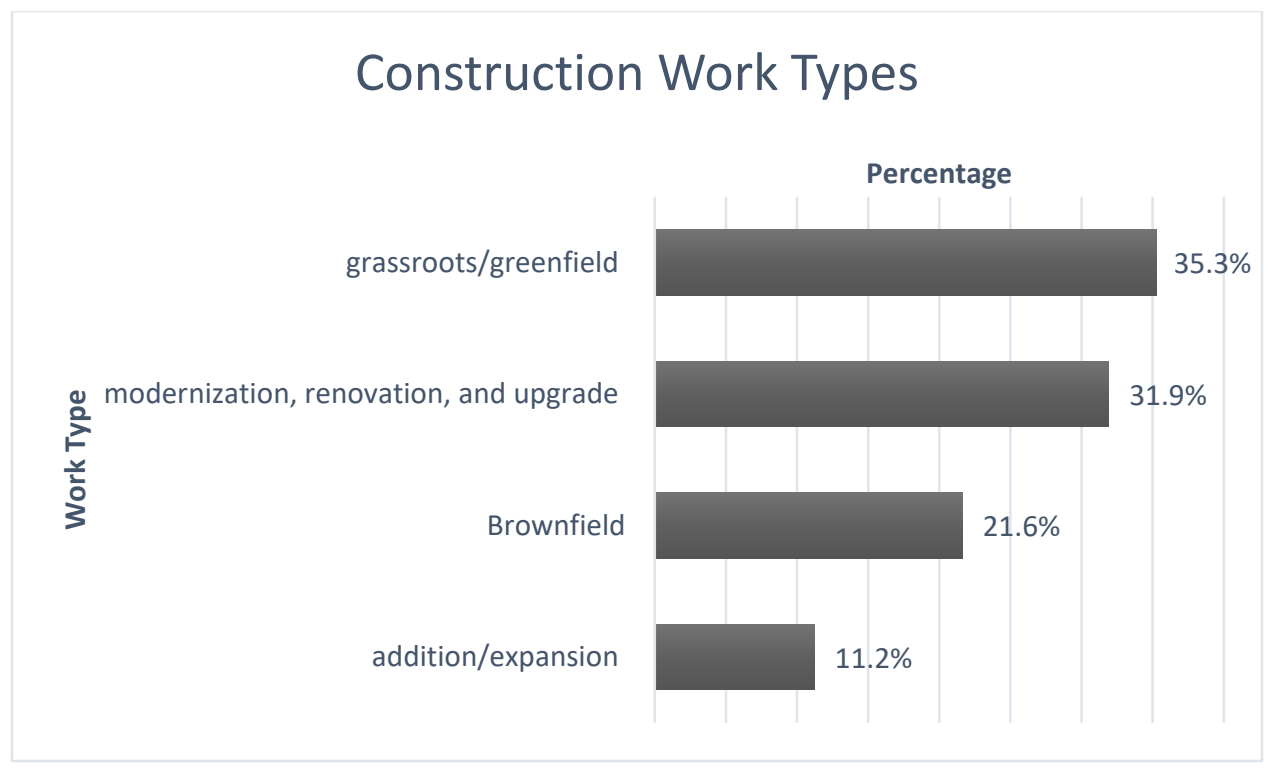

Figure 14: Construction work type of studied projects

\subsubsection{Owner Types}

Most of the respondents were from privately-owned projects, but some publicly-owned projects were also included, as shown in Figure 15.

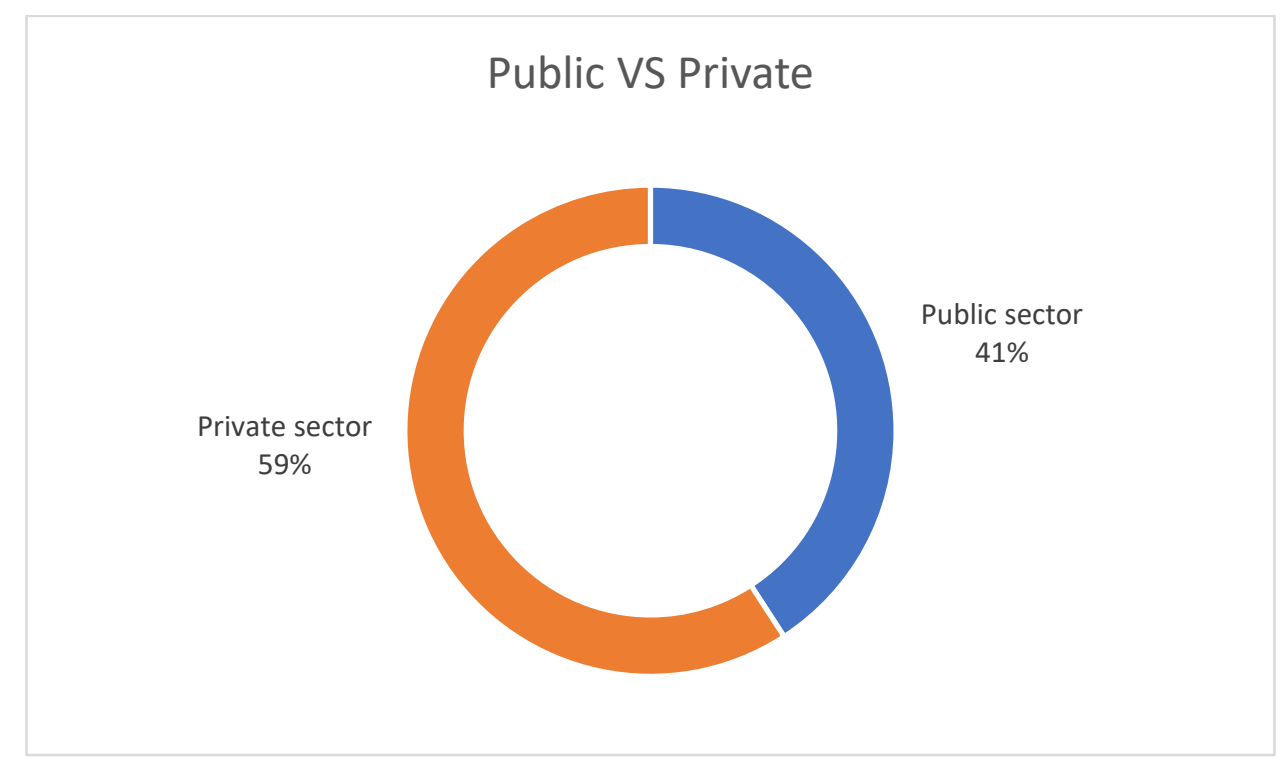

Figure 15: Owner type of studied projects 


\subsubsection{Construction Pace}

Within this study, construction pace refers to either the use of a fast-track approach or traditional construction pacing. While no preference for fast-track construction was expressed, Figure 16 shows that $60.7 \%$ of the projects did use a fast track approach, compared to $39.3 \%$ of projects that used traditional construction pacing.

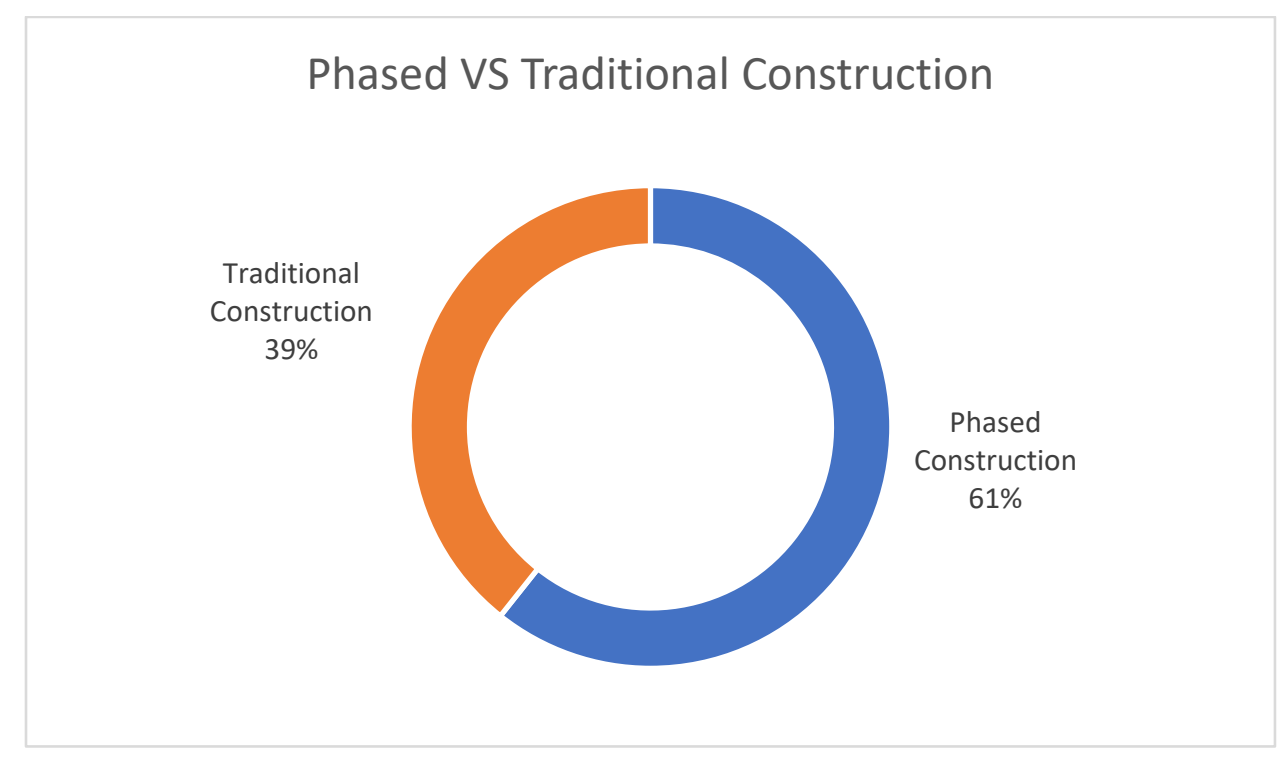

Figure 16: construction pace of studied projects

\subsubsection{Construction Completion Cost and Date Distribution}

Most of the studied projects were completed in the last five years (2014-2018). Figure 17 and Figure 18 show a summary of the distribution of the construction completion cost and duration, respectively. 


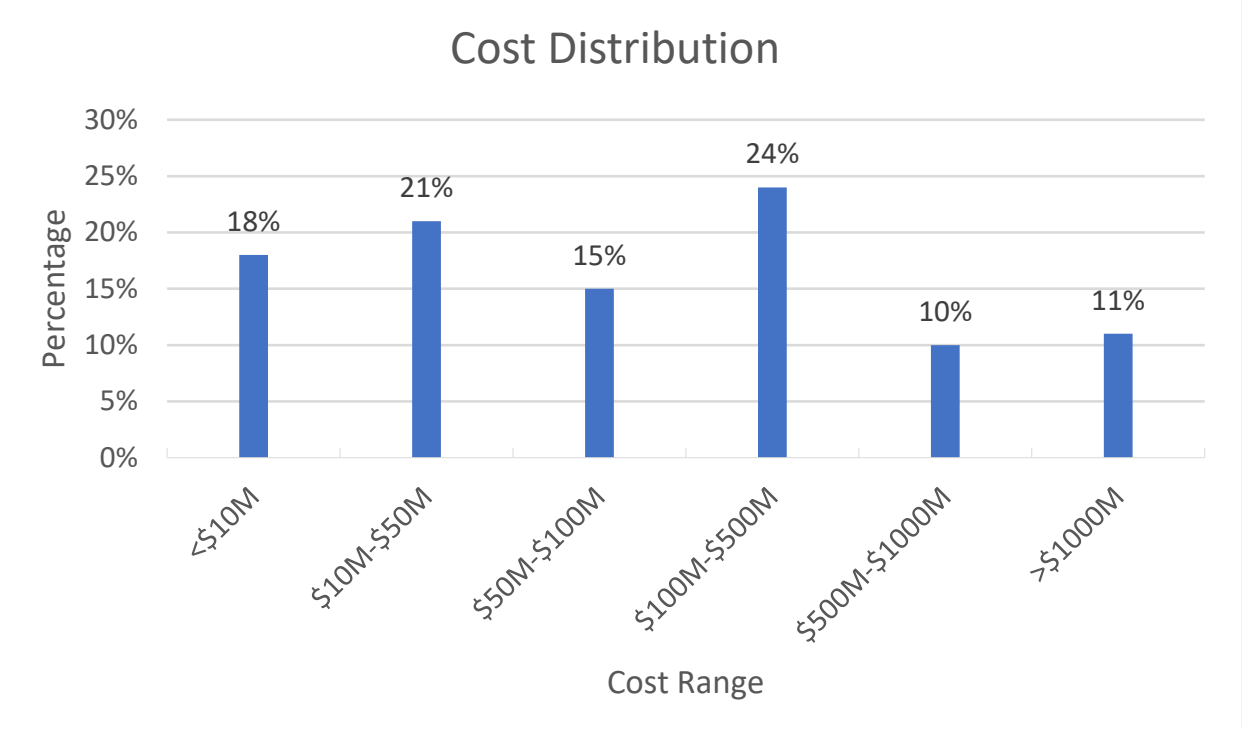

Figure 17: Construction cost of studied projects

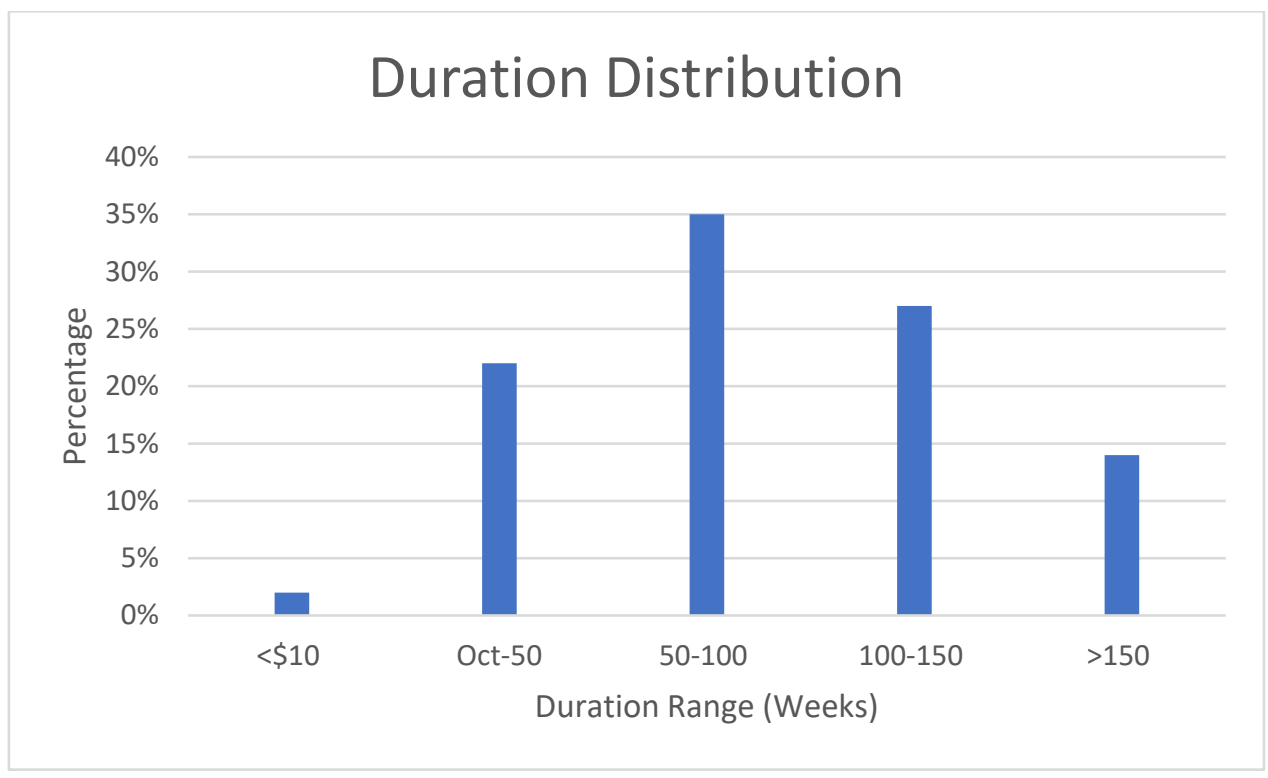

Figure 18: Construction duration of studied projects

\subsubsection{Project Delivery System Distribution}

Each of the major project delivery systems (PDSs) was represented in the dataset as shown in Figure 19. The most represented PDS was Design-Bid-Build (DBB), with 27.5 \% of projects. Design-Build (DB) (25\%) was the next most prevalent, and Integrated Project Delivery (IPD), 
Construction Management-Agent (CM-agent) and Construction Management At-Risk (CMAR) were also represented having 18.3\%, 15.8\%, and 7.5\%, representation, respectively. Other types of delivery systems such as Engineering Procurement and Construction (EPC), Engineering Procurement Construction and Management (EPCM), and Engineering, Procurement, Construction and Fabrication (EPCF) constituted 5.8\% of the projects.

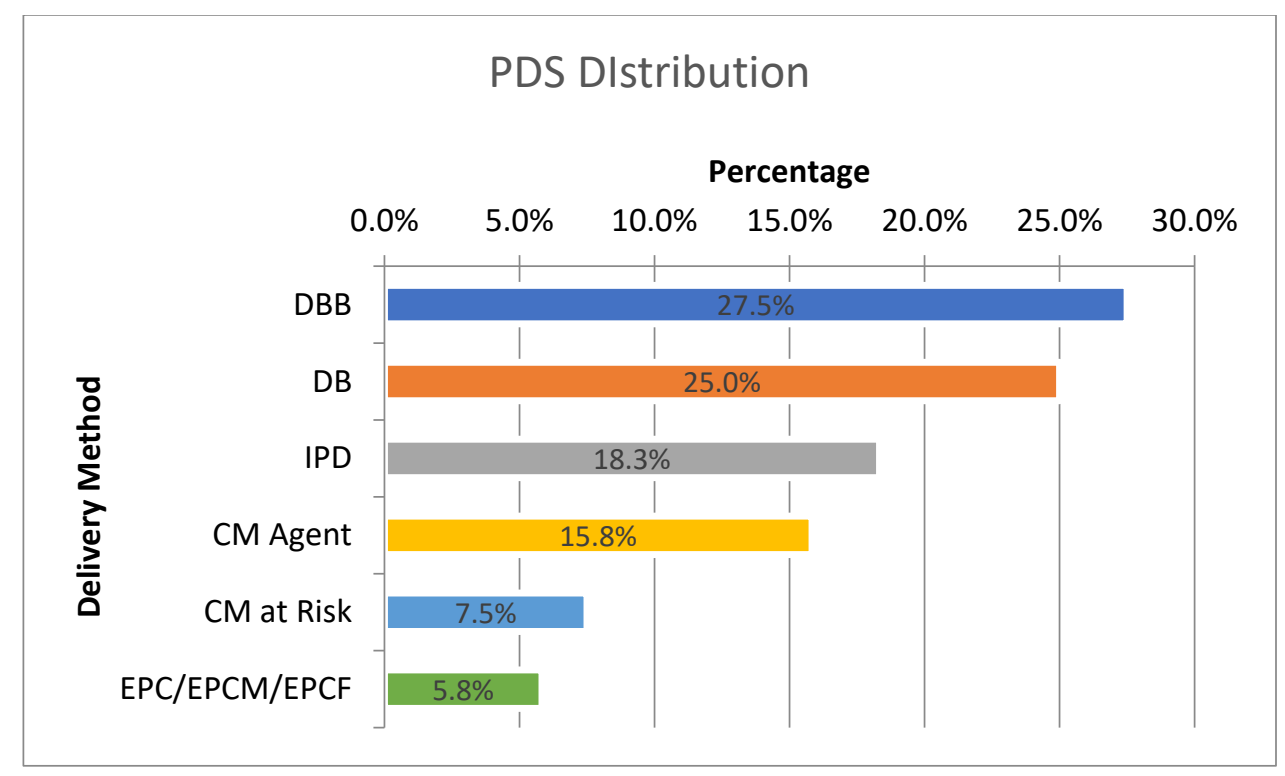

Figure 19: Project delivery system of studied projects

\subsubsection{Contract Type Distribution}

The lion's share of projects in this study was delivered under lump-sum contracts (43.7\%), while the second most prevalent contract type was cost-plus-fee at $21.8 \%$. Time and material (T\&M), guaranteed maximum price (GMP) and unit price constituted 20.2\%, 7.6\%, and 6.7\%, respectively. Figure 20 shows the distribution of the contract type of studied projects. 


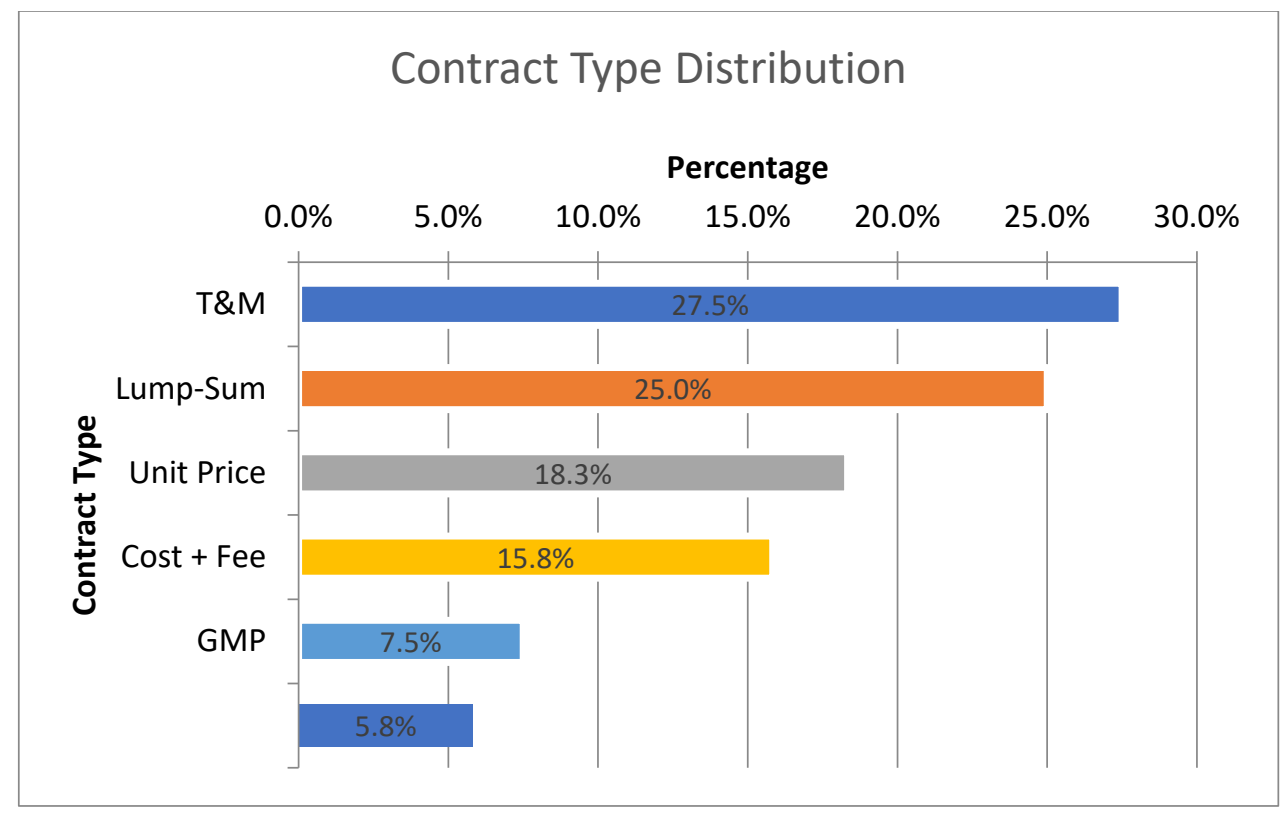

Figure 20: Contract type of studied projects 


\subsection{Summary and Conclusions}

This chapter presented and defined a comprehensive list of the variables used in this research and provided context for their selection where necessary. These variables include the post-lagging performance metrics used to assess the project success/failure and competency factors that can apply to virtually every type of construction project. Further, these variables form the basis of the quantitative scoring system and a delineation between successful and less-than successful projects, which will be discussed in forthcoming chapters. In addition, the mechanism of data collection (survey) was discussed and the characteristics of the dataset were presented. 


\section{Chapter 4 Quantitative Model of Project Performance}

Chapter 4 discusses the development of the mathematical formulation of the Project Performance Assessment (PPA) score. The objectives of this chapter are to 1) provide a measurement of project success for different projects; 2) merge the key metrics that affect project performance into one unique cumulative score that can be used to compare different projects; 3) benchmark successful projects from less-than-successful projects by performing K-means clustering; and 4) identify objective data-based weights for these metrics, and therefore, alleviate the subjectivity associated with experts' judgment regarding their relative importance to one another.

\subsection{Statistical/Mathematical Methodology}

The first stage of developing the mathematical formulation is to check the multicollinearity, which is the occurrence of high intercorrelations among independent variables in a mathematical/statistical model. Multicollinearity can lead to skewed or misleading results when a researcher or analyst attempts to determine how each independent variable can be used most effectively to predict or understand the dependent variable in a mathematical/statistical model. In other words, multicollinearity can exist when two independent variables are highly correlated. It can also occur if an independent variable is computed from other variables in the data set or if two independent variables provide similar and repetitive results. In sum, multicollinearity can lead to wider confidence intervals and less reliable probability values for the independent variables. That is, the statistical inferences from a model with multicollinearity may not be dependable. One of the most common ways of eliminating multicollinearity is to first identify collinear independent variables and then remove all but one. This is performed by using a pairwise correlation, which 
allows the user to see the correlation between their variables in a tabular or visual format. In this scenario, there is no universal cutoff, but in this dissertation, a pairwise correlation was conducted to remove performance metrics with correlations coefficients $>0.75$ or $<-0.75$.

The figure below shows the pairwise correlation matrix for performance metrics. These results show that there is no occurrence of high intercorrelations among independent variables in accordance with the cutoff value.

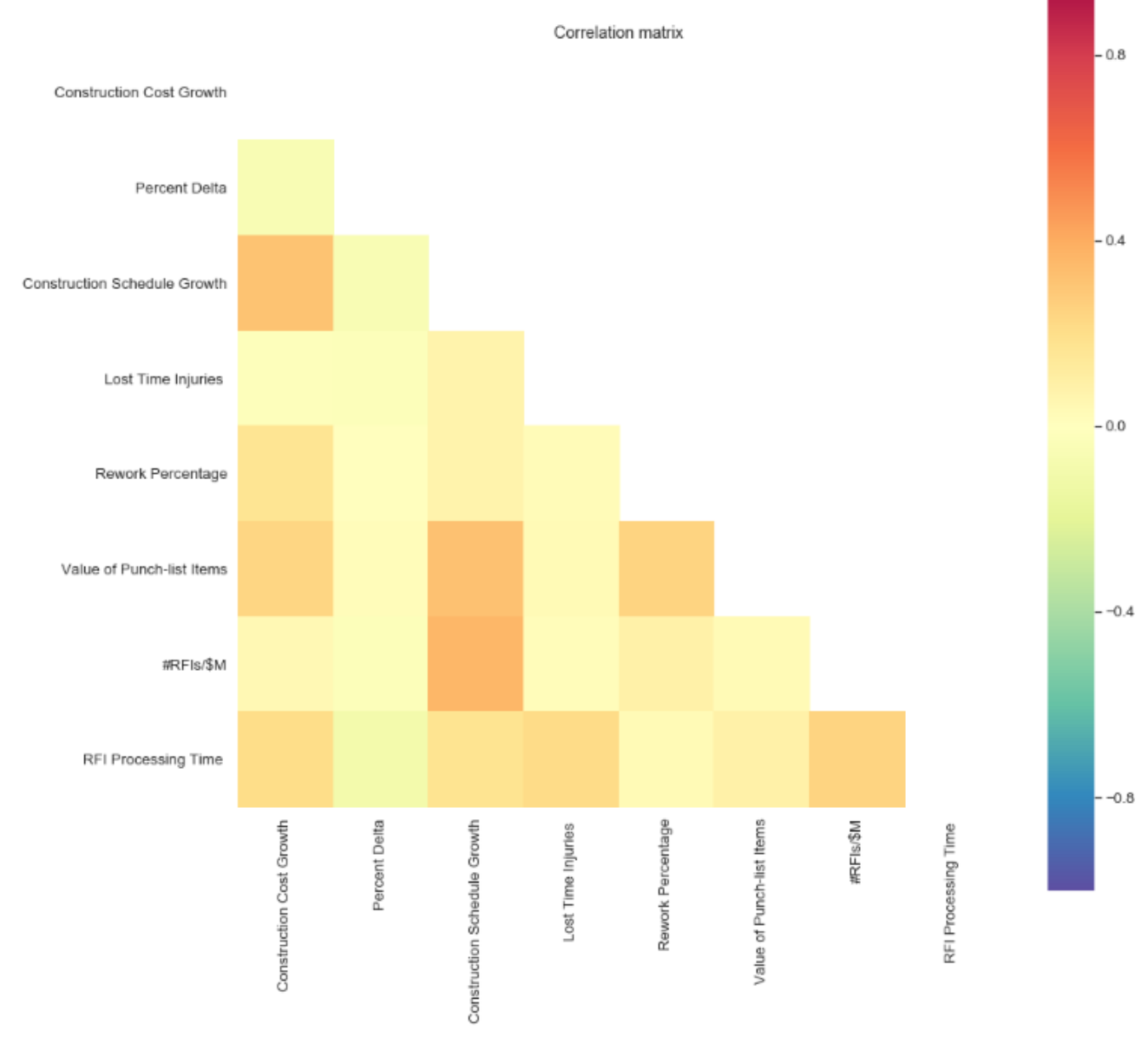

Figure 21: Correlation Matrix for Performance Metrics 


\subsection{Introduction to First Mathematical Model}

The first mathematical model applied in this study was developed by Labib (2019). This model is a newly developed dimensionality reduction technique, which performs linear mappings of data to a lower-dimensional space in such a way that a sum of squared correlations is maximized between the data in the high-dimensional representation with its output in the lower-dimensional space. In the context of this analysis, to fulfill the objectives, this mathematical model uses a sum of squared correlations approach to compute weights for the PPA score, such that the weights generated by this model return the maximum possible sum of squared correlations between the vector of the discrete performance metrics and that of the PPA.

\subsubsection{The PPA Mathematical Model}

This section provides a thorough explanation of and elaboration on the derivation of the first mathematical formulation. In the context of this dissertation, it will be used to derive the PPA score for the different projects by combining the previously mentioned eight key performance metrics spanning over six performance areas, which will be discussed in this chapter. The first mathematical model will also be used to rank the relative impact of the competency factors within each's category and compute a customized competency category-specific score from various competency factors spanning nine categories, which will be further discussed in chapter 5 .

Given a set of $m$ projects, each having scores of $n$ factors, then let $x_{i j}$ denote the score of factor $i$ for project $j$, where $1 \leq i \leq n$ and $1 \leq j \leq m$. To compare the relative importance of these factors, we use the standardized scores $\mathrm{z}_{i j}$ by normalizing each factor - subtracting each data value from its variable's measured mean $\left(\bar{x}_{i}\right)$. Hence, its empirical mean (average) is zero and when 
dividing it by the standard deviation $\left(s_{i}\right)$, then the variable's variance equals to 1 , as given by the following equation:

$$
z_{i j}=\frac{x_{i j}-\bar{x}_{i}}{ \pm s_{i}}
$$

where:

$$
\bar{x}_{i}=\frac{1}{m} \sum_{i=1}^{m} x_{i j} \quad \text { and } \quad s_{i}^{2}=\frac{1}{m-1} \sum_{i=1}^{m}\left(x_{i j}-\bar{x}_{i}\right)^{2}
$$

A positive sign is used if factor $i$ is a goodness factor, and a negative sign is used if it is a poor performance factor.

The objective of this model is to derive the following linear weighted function of the standardized factors of the projects:

$$
S\left(z_{1}, z_{2}, \ldots, z_{n}\right)=w_{1} z_{1}+w_{2} z_{2}+\cdots+w_{n} z_{n}
$$

Where the weights $w_{i}$ are selected in such a way as to maximize the length of the vector, its components are the correlations between the overall score vector:

$$
S=\left[\begin{array}{c}
S_{1} \\
S_{2} \\
\vdots \\
S_{m}
\end{array}\right]=\left[\begin{array}{cccc}
z_{11} & z_{12} & \cdots & z_{1 n} \\
z_{21} & z_{22} & \cdots & z_{2 n} \\
\vdots & \vdots & \ddots & \vdots \\
z_{m 1} & z_{m 2} & \cdots & z_{m n}
\end{array}\right]\left[\begin{array}{c}
w_{1} \\
w_{2} \\
\vdots \\
w_{n}
\end{array}\right]=Z W
$$

of the $m$ projects and the standardized scores of each of the $n$ factors: $Z_{i}=\left[\begin{array}{c}z_{i 1} \\ z_{i 2} \\ \vdots \\ z_{i m}\end{array}\right]$. Moreover, the weights should add up to 1 . In other words, the quantity needs to be maximized:

$$
\sum_{i=1}^{n}\left[\operatorname{corr}\left(S, Z_{i}\right)\right]^{2}
$$




$$
\text { subject to: } \sum_{i=1}^{n} w_{i}=1
$$

In Labib (2019), it was shown that the weight vector $W$ satisfying (4) is an eigenvector of the correlation matrix $Z^{T} Z$ corresponding to its maximum eigenvalue. Thus, to satisfy (5), $W$ can be calculated by getting any such eigenvector $V$, and letting $W=V /\left(\left[\begin{array}{lll}1 & 1 & \ldots\end{array}\right] V\right)$.

\subsubsection{Project Performance Assessment (PPA) Results}

Based on the mathematical model, the final weights of the eight performance metrics were identified as seen in Figure 21. The results showed that the top three metrics are: construction schedule growth, construction cost growth, and \% delta having 18.43\%, 17.38\%, and 15.22\%, respectively. On the other hand, the percentage of rework and the number of lost-time injuries per 1 million dollars have the lowest contribution to calculating the PPA score. While a lower value does not simply correlate to a low degree of importance, that importance depends is contextual, depending on the correlation strength between each metric as a vector and the PPA score vector. This can also be explained as such, the sample of the projects in this model has very few numbers of rework activities and lost-time injuries.

To simplify the computations for the users, the factors were reverted from their standardized values to their original values using the mean and standard deviation as shown in the following equations:

$$
\mathrm{PPA}=\sum_{i=1}^{n} w_{i} z_{i}=\sum_{i=1}^{n} w_{i}\left(\frac{x_{i j}-\bar{x}_{j}}{ \pm s_{j}}\right)=\sum_{i=1}^{n}\left(\frac{w_{i}}{ \pm s_{j}}\right) x_{i j}-\sum_{i=1}^{n}\left(\frac{w_{i} \bar{x}_{j}}{ \pm s_{j}}\right)=\beta+\sum_{j=1}^{n} \alpha_{j} x_{i j}
$$

Where 


$$
\alpha_{j}=\frac{w_{i}}{ \pm s_{j}} \text { and } \beta=\frac{w_{i} \bar{x}_{j}}{ \pm s_{j}}
$$

A simplified equation was developed to accommodate the unstandardized data. Therefore, the aggregation of this transformation for the eight performance metrics resulted in the constant term in the following equation:

PPA Standardized Score $=$

0.855 - 0.371 Construction Cost Growth - 0.107 Percent Delta

- 0.521 Construction Schedule Growth

- 0.044 Lost Time Injuries per Million Dollars

- 0.489 Rework Percentage - 0.142 Value of Punch List Items

- 0.001 Number of RFIs per Million Dollars

- 0.122 RFI Processing Time

The results of the PPA score are in a standardized form, i.e. a value that can be positive or negative. For better visualization and comparison of projects to one another, the authors have decided to scale the generated PPA score to have a range between 5\% to 95\%. A range of [5, 95\%] was chosen instead of [0,100\%], as it is not reasonable to presume the worst possible project performance to be $0 \%$ and the best possible project performance to be $100 \%$. Therefore, the equation below eliminates errors that stem from standardization while also providing a userfriendly methodology to compute the final PPA score of a range between $5 \%$ to $95 \%$ :

$$
P P A_{\text {final }}=\frac{P P A_{i}-P P A_{\text {min }}}{P P A_{\max }-P P A_{\min }}(0.95-0.05)+0.05
$$

where

$$
P P A_{\text {min }}=\min \left\{P P A_{i} \mid 1 \leq i \leq m\right\} \quad \text { and } \quad P P A_{\max }=\max \left\{P P A_{i} \mid 1 \leq i \leq m\right\}
$$

The final PPA score equation can be written in this form: 


$$
\begin{aligned}
P P A_{\text {final }} & =0.05+\left(\frac{P P A_{i}+2.302}{3.889}\right) \times 0.9 \\
& =0.583+0.231 \times P P A_{i}
\end{aligned}
$$

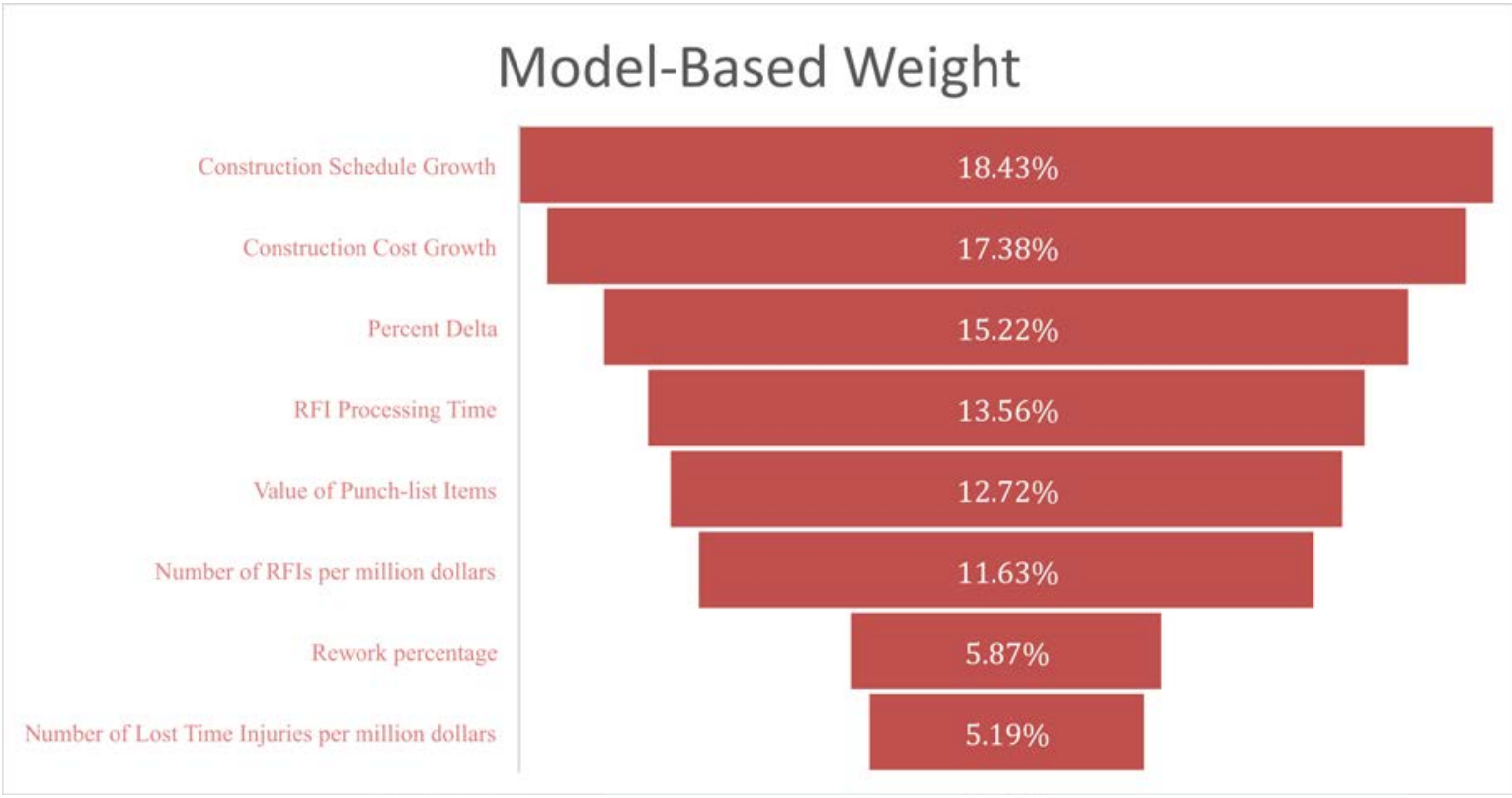

Figure 22: Weights breakdown of the PPA metrics

\subsection{Cluster Analysis}

\subsubsection{Introduction to Cluster Analysis}

Cluster analysis is a statistical method that comprises a range of numerical methods for classifying multivariate data into subgroups (or clusters). Cluster analysis is also referred to as segmentation analysis, taxonomy analysis, or clustering.

The purpose of cluster analysis is to sort different objects or data points into groups with the common goal of uncovering or discovering clusters of observations that are homogeneous and separated from other groups. To put another way, cluster analysis is used to group a set of projects based on their dissimilarity to create clusters with maximum inter-cluster distance (distance 
between points in different clusters) and minimum intra-cluster (distance between points within a cluster).

\subsubsection{Benefits of Cluster Analysis}

Cluster analysis differs from many other statistical methods because it is mostly used when researchers do not have an assumed principle or fact for the foundation of their research. Clustering also allows researchers to identify and define patterns between data elements. Revealing these patterns between data points helps to distinguish and outline structures that might not have been apparent before, and thus gives significant meaning to this discovered data. Once a clearly defined structure emerges from the dataset at hand, informed decision-making becomes much easier.

This analysis technique is typically performed during the exploratory phase of research, because unlike techniques such as factor analysis, it does not distinguish between dependent and independent variables. Instead, cluster analysis is mostly leveraged to discover structures in data without providing an explanation or interpretation. Clustering techniques have proven useful in a wide range of areas such as medicine, psychology, bioinformatics, and market research. For example, when cluster analysis is performed in market research, specific groups can be identified within a population. The analysis of these groups can then determine how likely a population cluster is to purchase products or services. If these groups are defined clearly, a marketing team can then target the various clusters with tailored, targeted communication. 


\subsubsection{Types of Cluster Analysis}

Cluster analysis techniques are described in detail by Gordon $(1987,1999)$ and Everitt, Landau, Leese, and Stahl (2011). There are two primary methods used to perform cluster analysis: agglomerative hierarchical clustering techniques and Partitioning-based clustering.

\subsubsection{Hierarchical Clustering}

This is a common method of clustering that produces a hierarchical classification of data. In a hierarchical classification, the data are not partitioned into a particular number of classes or groups at a single step. Instead, it creates a series of models with cluster solutions from 1 (all cases in one cluster) to n (each case is an individual cluster). Hierarchical clustering can group variables in a manner similar to factor analysis. Lastly, hierarchical cluster analysis can handle nominal, ordinal, and scale data.

\subsubsection{2. $\quad$ Partitioning-based Clustering}

The most popular class of clustering algorithms is the partitioning-based clustering. These algorithms are used to classify observations within a data set into multiple groups based on their similarity. The algorithms require the analyst to specify the number of clusters to be generated and then use an iterative approach to relocate data points between clusters until an optimal partition is attained. The commonly used partitional clustering includes:

1. K-means clustering (MacQueen 1967), in which each cluster is represented by the center or means of the data points belonging to the cluster. The K-means method is sensitive to anomalous data points and outliers. 
2. K-medoids clustering or PAM (Partitioning Around Medoids, Kaufman \& Rousseeuw, 1990), in which each cluster is represented by one of the objects in the cluster. PAM is less sensitive to outliers compared to k-means.

3. CLARA algorithm (Clustering Large Applications), which is an extension to PAM adapted for large data sets.

\subsection{K-Means Clustering}

The mathematical model in the previous section was used to calculate the PPA score for each of the 120 projects. Afterward, the author sought to benchmark successful projects from lessthan-successful projects by performing K-means clustering, a common method for the partitioning clustering analysis technique. K-means clustering is a commonly used unsupervised machine learning algorithms that can quickly cluster large datasets. It can be also referred as centroid-based clustering, in which it is used to group a set of projects in such a way that projects in the same group (a cluster) behave similarly to each other; while if the difference between the project performance and the other projects' performance already in the cluster is significant, then the project is assigned to a different cluster. The algorithm starts by randomly selecting the number of clusters prior to performing the actual study. In general, the k-means method will produce exactly k different clusters. The main idea is to define k centroids, one for each cluster. These centroids should be placed as far away from each other as possible. The next step is to take each point belonging to a given data set and associate it with the nearest centroid. When no point is pending, the positions of the k centroids are recalculated. This procedure is repeated until the centroids no longer move. 
The algorithm tries to find groups by minimizing the distance between the observations, which are called local optimal solutions. The distances are measured based on the coordinates of the observations. The algorithm works as Figure 22.

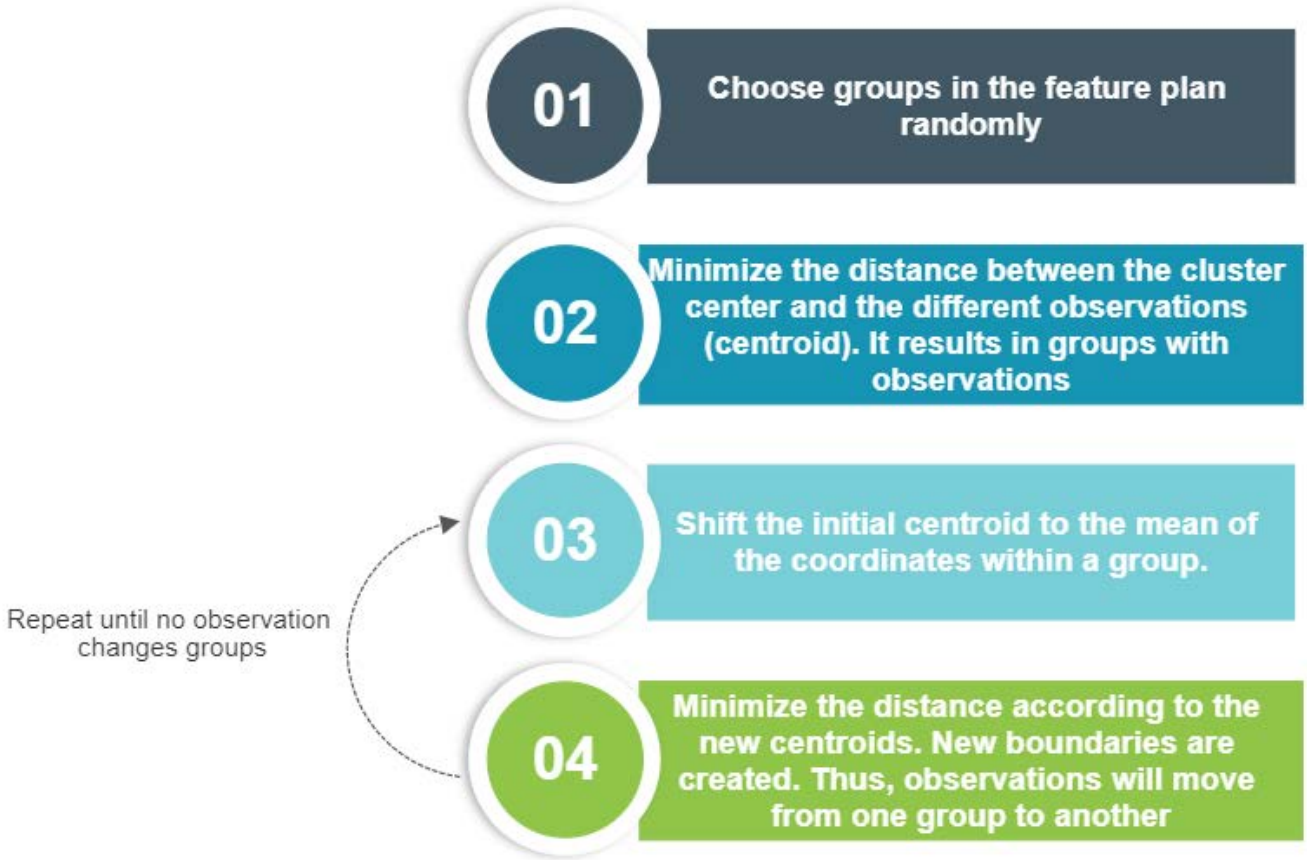

Figure 23: Cluster analysis algorithm

\subsection{Clustering Distance Measures}

The classification of observations into groups requires some methods for computing the distance or the dissimilarity between each pair of observations. The result of this computation is known as a dissimilarity or distance matrix. There are many methods to calculate this distance information; the choice of distance measurement is a critical step in clustering because it defines how the similarity of two elements (x,y) is calculated and it will influence the shape of the clusters. Importantly, the centroid is defined as the "center" of each cluster and often used as a representative point (for each cluster). Denoted $S_{k}$ where $\mathrm{k}$ is the cluster index and computed as the mean of all points in a cluster: 


$$
S_{k}=\frac{1}{\left|S_{k}\right|}=\sum_{i \in S_{k}} x_{i}
$$

The classical methods for distance measures are Euclidean and Manhattan distances, which are defined as follows:

- Euclidean: represents "straight line distance” and is the most commonly used distance metric. Also known as the $l_{2}$ norm:

$$
d\left(x_{1}, x_{2}\right)=\left\|x_{1}-x_{2}\right\|_{2}=\sqrt{\sum_{f=1}^{F}\left(x_{1 f}-x_{2 f}\right)^{2}}
$$

- Manhattan: represents the absolute value of differences. Also known as the $l_{1}$ norm:

$$
d\left(x_{1}, x_{2}\right)=\left\|x_{1}-x_{2}\right\|_{1}=\sum_{f=1}^{F}\left|x_{1 f}-x_{2 f}\right|
$$

- Chebyshev: represents the maximum difference. Also known as the $l_{\infty}$ norm:

$$
d\left(x_{1}, x_{2}\right)=\left\|x_{1}-x_{2}\right\|_{\infty}=\max _{f=1, \ldots, F}\left|x_{1 f}-x_{2 f}\right|
$$

- Minkowski: is a generalization of the Euclidean, Manhattan, and Chebyshev methods.

Also known as the $l_{P}$ norm:

$$
d\left(x_{1}, x_{2}\right)=\left\|x_{1}-x_{2}\right\|_{P}=\left(\sum_{f=1}^{F}\left(x_{1 f}-x_{2 f}\right)^{P}\right)^{\frac{1}{P}}
$$

- Hamming: counts the number of features that are different:

$$
d\left(x_{1}, x_{2}\right)=\sum_{f=1}^{F} \|\left(x_{1 f} \neq x_{2 f}\right)
$$


where $\|$ is an indicator that is 1 if the condition $\left(x_{1 f} \neq x_{2 f}\right)$ is true, and otherwise is 0 .

\subsubsection{Determining the Optimal Number of Clusters}

Determining the optimal number of clusters in a dataset is a fundamental part of partitioning clustering, such as k-means clustering, which requires the user to specify the number of clusters $\mathrm{k}$ to be generated. Unfortunately, there is no definitive answer to this question. The optimal number of clusters is partially subjective and depends on the method used for measuring similarities and the parameters used for partitioning. This section will describe different methods for determining the optimal number of clusters for k-means. These methods include direct methods and statistical testing methods:

1. Direct methods: consist of optimizing a criterion, such as the within-cluster sums of an absolute value of differences or the average silhouette. The corresponding methods are named elbow and silhouette methods, respectively.

2. Statistical testing methods: comparing evidence against a null hypothesis. An example is the gap statistic.

\subsubsection{Elbow Method}

Recall that the basic idea behind partitioning methods, such as k-means clustering, is to define clusters such that the total intra-cluster variation is minimized. The elbow method is a heuristic method of interpretation and validation of consistency within-cluster analysis designed to help find the appropriate number of clusters in a dataset.

This method looks at the percentage of variance explained as a function of the number of clusters: One should choose a number of clusters so that adding another cluster does not provide better modeling of the data. More precisely, if one plots the percentage of variance explained by 
the clusters against the number of clusters, the first clusters will add a lot of information (explains high variance), but at some point, the marginal gain will drop, giving an angle in the graph. The number of clusters is chosen at this point, hence the "elbow criterion." The percentage of variance explained is the ratio of the between-group variance to the total variance, also known as an F-test. A slight variation of this method plots the curvature of the within-group variance

The elbow method looks at the total of the absolute value of differences as a function of the number of clusters: One should choose a number of clusters so that adding another cluster does not improve better than the total of the absolute value of differences. The optimal number of clusters can be defined as follows:

1. Compute clustering algorithm (e.g., k-means clustering) for different values of k. For instance, by varying $\mathrm{k}$ from 1 to 10 clusters.

2. For each $\mathrm{k}$, calculate the total of the absolute value of differences.

3. Plot the curve of the total of the absolute value of differences according to the number of clusters k.

4. The location of a bend (knee) in the plot is generally considered as an indicator of the appropriate number of clusters.

The optimal number of clusters is found to be two clusters, as shown in Figure 23. 


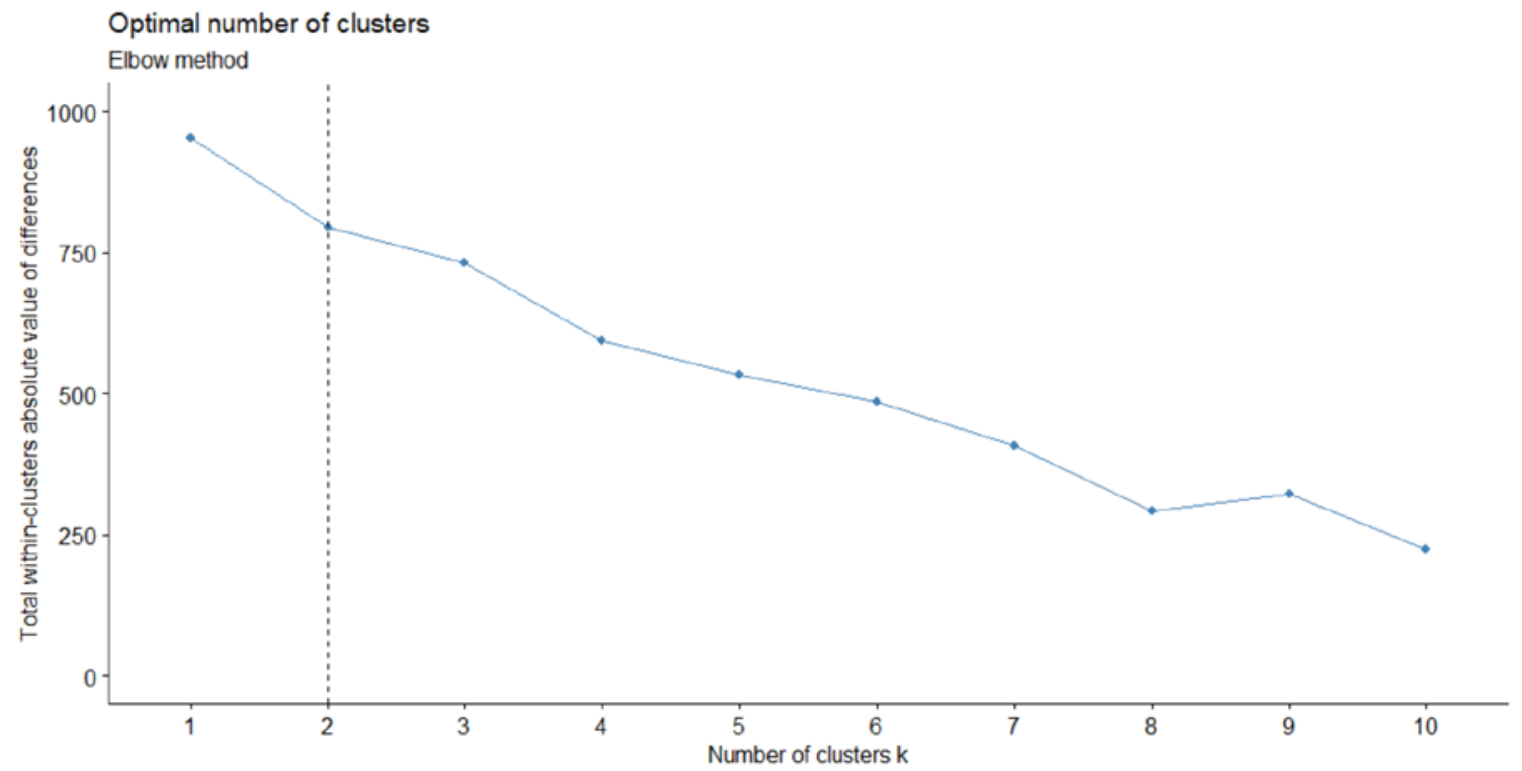

Figure 24: Elbow method plot

\subsubsection{Average Silhouette Method}

The average silhouette method measures the quality of a clustering. That is, it determines how well each object lies within its cluster. A high average silhouette width indicates a good clustering. The average silhouette method computes the average silhouette of observations for different values of $\mathrm{k}$. The optimal number of clusters $\mathrm{k}$ is the one that maximizes the average silhouette over a range of possible values for k (Kaufman and Rousseeuw 1990). The algorithm is similar to the elbow method and can be computed as follows:

1. Compute clustering algorithm (e.g., k-means clustering) for different values of k. For instance, by varying $\mathrm{k}$ from 1 to 10 clusters.

2. For each $\mathrm{k}$, calculate the average silhouette of observations (avg. sil).

3. Plot the curve of avg. sil according to the number of clusters $\mathrm{k}$.

4. The location of the maximum is considered as the appropriate number of clusters. 


\subsection{Silhouette Coefficient}

For each observation $i$, the silhouette width $s_{i}$ is calculated as follows:

1. For each observation $i$, calculate the average dissimilarity $a_{i}$ between $i$ and all other points of the cluster to which $i$ belongs.

2. For all other clusters $C_{i}$ to which $i$ does not belong, calculate the average dissimilarity $d(i, C)$ of $i$ to all observations of $C$. The smallest of these $d(i, C)$ is defined as $b_{i}=\min _{C} d(i, C)$. The value of $b_{i}$ can be seen as the dissimilarity between $i$ and its “neighbor” cluster, i.e., the nearest one to which it does not belong.

3. Finally, the silhouette width of the observation $i$ is defined by the formula:

$$
S_{i}=\frac{b_{i}-a_{i}}{\max \left(a_{i}, b_{i}\right)}
$$

Silhouette width can be interpreted as follows:

- $\quad$ Observations with a large $S_{i}$ (almost 1 ) are very well clustered.

- A small $S_{i}$ (around 0) means that the observation lies between two clusters.

- Observations with a negative $S_{i}$ are probably placed in the wrong cluster.

The optimal number of clusters is found to be two clusters, as shown in Figure 24. 


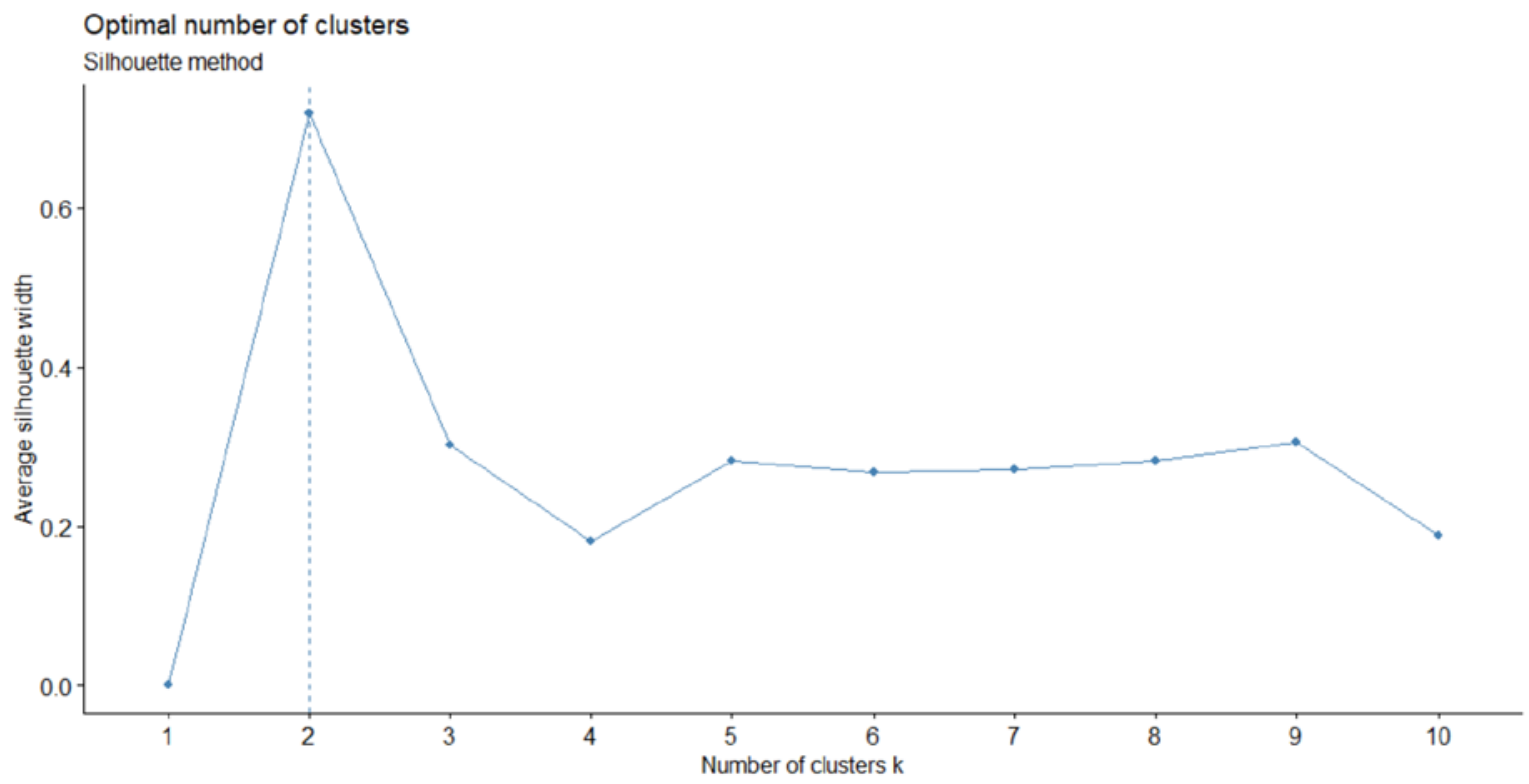

Figure 25: Average silhouette method plot

\subsubsection{Gap Statistic Method}

The gap statistic compares the total within intra-cluster variation for different values of $\mathrm{k}$ with their expected values under a null reference distribution of the data. The estimate of the optimal clusters will be the value that maximizes the gap statistic (i.e., that yields the largest gap statistic). This means that the clustering structure is far away from the random uniform distribution of points.

The algorithm works as follows:

1. Cluster the observed data, varying the number of clusters from $\mathrm{k}=1, \ldots, K_{\max }$, and compute the corresponding total within intra-cluster variation $W_{k}$.

2. Generate B reference data sets with a random uniform distribution. Cluster each of these reference data sets with varying number of clusters $\mathrm{k}=1, \ldots, K_{\max }$, and compute the corresponding total within intra-cluster variation $W_{k b}$. 
3. Compute the estimated gap statistic as the deviation of the observed $W_{k}$ value from its expected value $W_{k b}$ under the null hypothesis: $\operatorname{Gap}(k)=\frac{1}{B} \sum_{i b=1}^{B} \log \left(W_{k b}^{*}\right)-\log \left(W_{k}\right)$. Also compute the standard deviation of the statistics.

4. Choose the number of clusters as the smallest value of k such that the gap statistic is within one standard deviation of the gap at $k+1: \operatorname{Gap}(k) \geq \operatorname{Gap}(k+1)-S_{k+1}$

The optimal number of clusters is found to be two clusters, as shown in Figure 25.

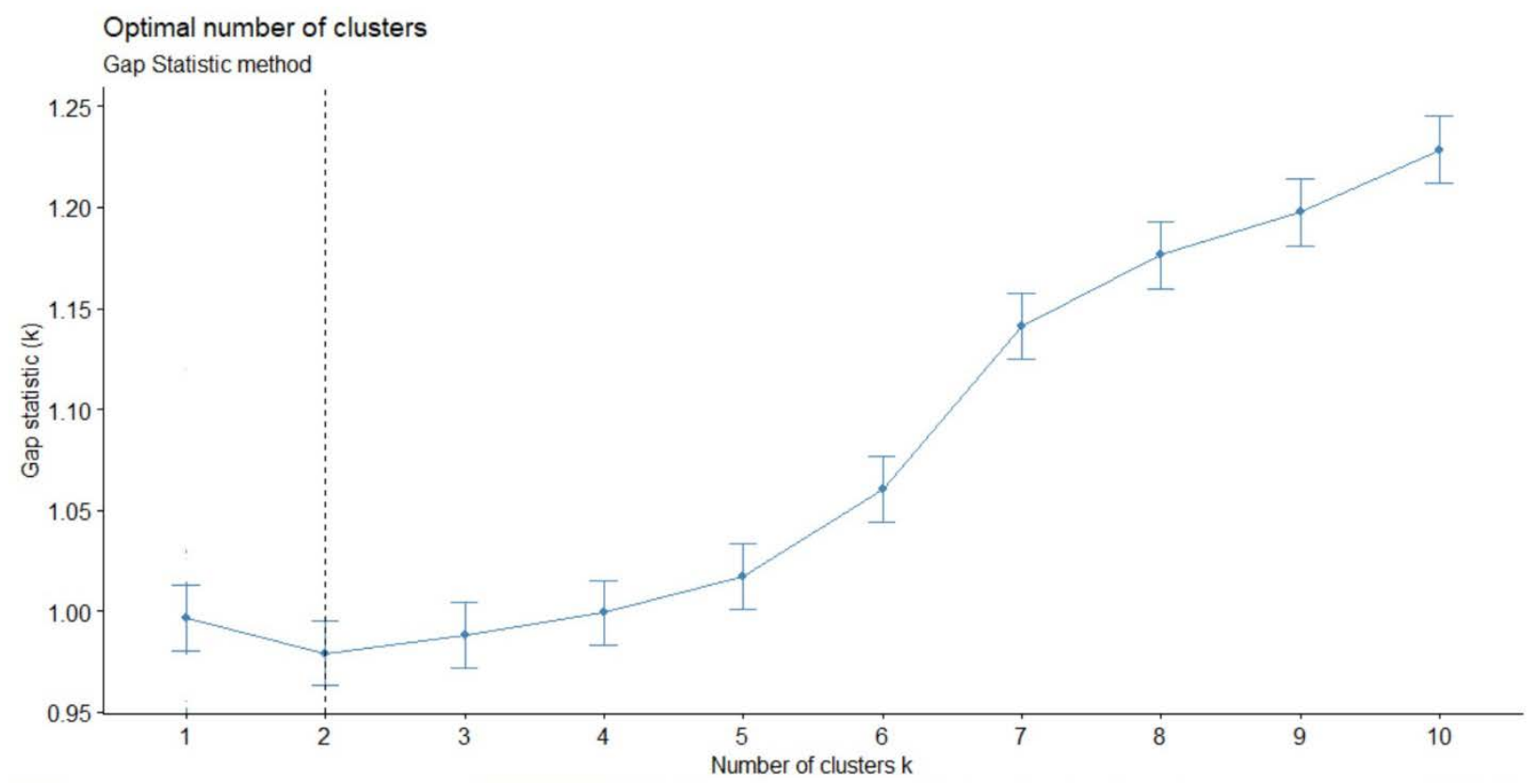

Figure 26: Gap statistic method plot

\subsubsection{Cluster Analysis Results}

The optimal number of clusters is found to be two clusters using the different methods, including the direct methods and statistical testing methods, where cluster 1 includes 78 projects cluster 2 encompasses the remaining 42 projects. The purpose of this stage is to perform a principal component analysis (PCA) and plot the data points according to the first two principal components 
that explain the majority of the variance, as shown in Figure 26. The first component and second component will be plotted on the $\mathrm{x}$-axis and the $\mathrm{y}$-axis, respectively. The plot reveals the distinct classification of projects with limited overlap between the clusters. It is found that cluster 1 has PPA scores with a range of 56\% - 95\%, while cluster 2 has PPA scores with a range of 5\% - 55\%. Therefore, it can be concluded that cluster 1 is defined as successful projects and cluster 2 is defined as less-than-successful projects.

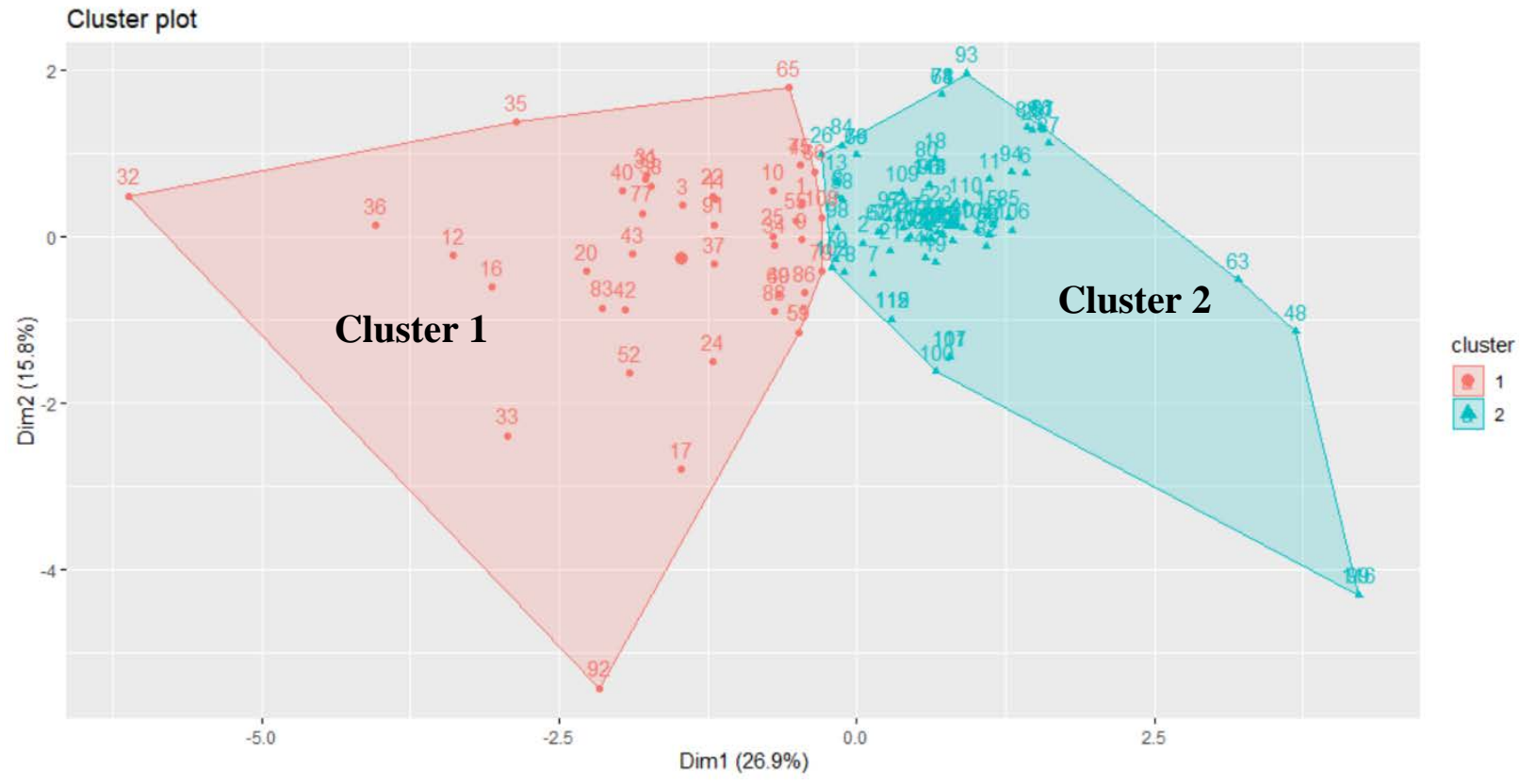

Figure 27: Cluster plot showcasing two data clusters 


\subsection{Project Performance Assessment (PPA) Score Analysis}

It is essential to understand the data type and validate the normality assumption in order to select the applicable significance tests. To assess the normality assumption, a Q-Q plot and
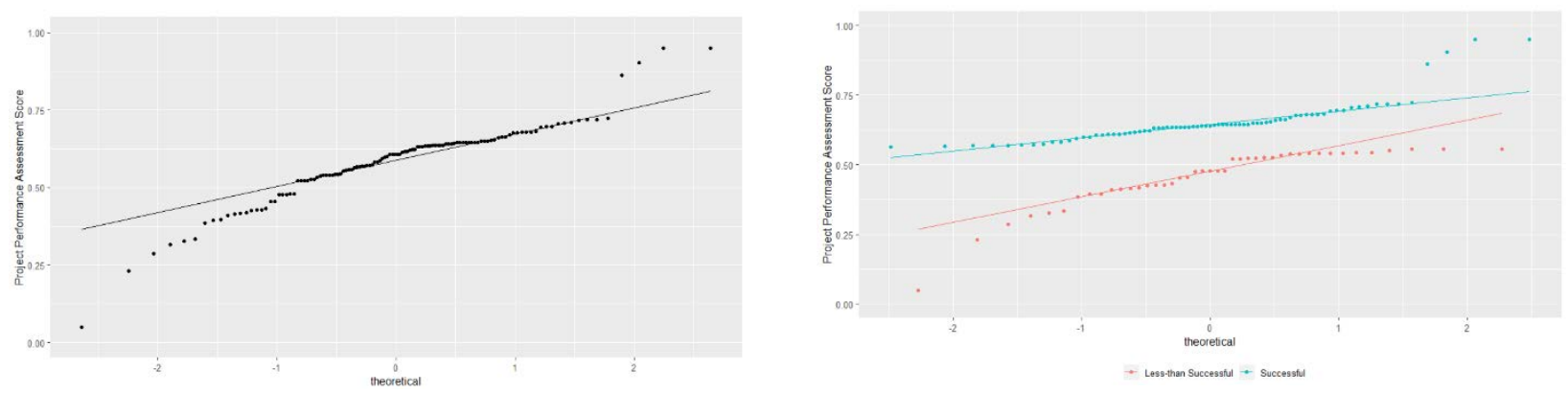

Figure 28: Q-Q plot of project performance assessment score

a Shapiro-Wilk test is conducted. The following Q-Q plot shows a clear departure from normality at the overall level of the dataset. As with the other analysis, the normality assumption is not valid, as it was confirmed at a 95\% confidence level by the Shapiro-Wilk test, which is shown in the table below.

Table 17: Shapiro-Wilk test for PPA scores

\begin{tabular}{ccc}
\hline \hline Parameter & $p$-value & Significance at a 95\% Confidence Level \\
\hline \hline Overall & 0.000 & Significant \\
Successful Projects & 0.000 & Significant \\
Less-than-successful Projects & 0.000 & Significant \\
\hline \hline
\end{tabular}




\subsection{Performance Metrics vs. PPA Scores/Project Status Assessment}

The developed mathematical model aims to maximize the sum of the square of the correlation between each of the individual performance metrics and PPA scores. Therefore, this analysis serves as an internal validation to verify that the selected metrics provide a substantial contribution to the PPA model by checking the correlation coefficient. In that sense, if a metric is found to have a negligible correlation coefficient with PPA scores, it can either be concluded that the metric is not representative of the project's performance, or the developed model was not successful in finding the optimum weights that could capture the maximum contribution of the introduced metrics. Note, the linear regression line is present for representation purposes only and does not contribute to the computation.

The dataset experienced a significant departure from the normality assumption, as will be shown later in the analysis. Therefore, Spearman's Correlation test was used to statistically test the correlation between each of the individual performance metrics and the PPA score. This is done by performing a hypothesis test for this correlation, such that the null hypothesis states that there is no correlation between each of the individual performance metrics and the PPA score, while the alternative hypothesis states that correlation exists. Using Spearman's Correlation test for hypothesis testing produces a $p$-value, which can be thought of as the probability of having no statistical correlation between each of the individual performance metrics and the PPA score. Accordingly, a $p$-value of less than 0.05 demonstrates that the data provides evidence to reject the null hypothesis at a 5\% significance level, thus concluding at a 95\% confidence level that there is a statistically significant correlation between each of the individual performance metrics and the PPA score. A second statistical metric is produced from this analysis is Spearman's Rho $\rho$ correlation coefficient, which measures the relationship between each of the individual 
performance metrics and the PPA score, so that it has a value of between -1 and 1 . A 0 means there is no relationship between the variables at all, while -1 or 1 means that there is a perfect negative or positive correlation.

For further analysis, a Fligner-Killeen test and Wilcoxon rank-sum test were performed to statistically test the relationship between the project status and each of the individual performance metrics. The Fligner-Killeen test checks for homogeneity of variance, when data are non-normally distributed or when problems related to outliers in the dataset cannot be resolved. The null hypothesis is that all variances are equal, while the alternative hypothesis is that all variances are unequal. Therefore, a resulting $p$-value under 0.05 means that the test produces significant results and the variances are not equal. For the Wilcoxon rank-sum test, the null hypothesis is that the levels of the dependent variable across the different levels of the independent variable are the same, whereas the alternative hypothesis is that the levels of the dependent variable across the different levels of the independent variable are dissimilar. This test also provides a $p$-value that can be thought of as the probability that the null hypothesis is correct. Accordingly, a $p$-value of less than 0.05 demonstrates that the data provides evidence to reject the null hypothesis at a $5 \%$ significance level, thus concluding at a 95\% confidence level that there is statistically significant evidence that the levels of the dependent variable significantly vary across the different levels of the independent variable.

\subsubsection{Construction Cost Growth}

A Q-Q plot and Shapiro-Wilk test are conducted to assess the normality assumption. The following Q-Q plot shows a clear departure from normality at the overall level of the dataset. 

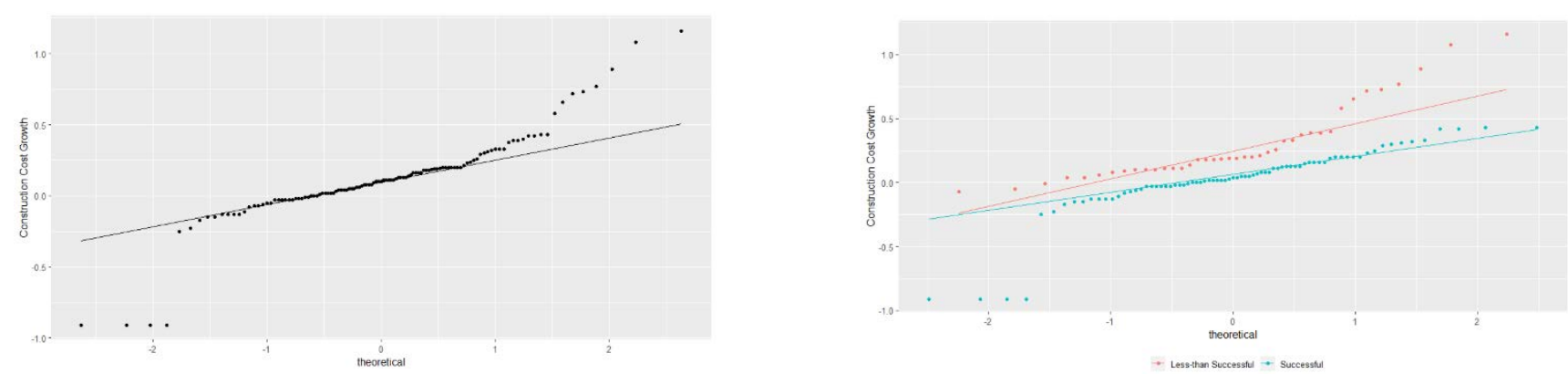

Figure 29: Q-Q Plot of construction cost growth

As with the other analysis, the normality assumption is not valid, as it was confirmed at a 95\% confidence level by the Shapiro-Wilk test, which is shown in the table below.

Table 18:Shapiro-Wilk test for construction cost growth

\begin{tabular}{ccc}
\hline \hline Parameter & $p$-value & Significance at a 95\% Confidence Level \\
\hline \hline Overall & 0.000 & Significant \\
Successful Projects & 0.000 & Significant \\
Less-than-successful Projects & 0.000 & Significant \\
\hline \hline
\end{tabular}

The following figure shows that there is a moderate to strong negative correlation between PPA scores and the construction cost growth. The correlation is statistically significant at a $95 \%$ confidence level with a p-value of 0.000 and a correlation coefficient of -0.68 . The plot chart also reveals a negative linear relationship for both successful and less-than-successful projects between PPA scores and the construction cost growth. However, it can be noticed that successful projects follow a sharper linear relationship as opposed to less-than-successful ones. For both successful 
and less-than-successful projects, the correlation is statistically significant at a $95 \%$ confidence level with a p-value of 0.000 and 0.0013 and a correlation coefficient of -0.84 and -0.60 , respectively.

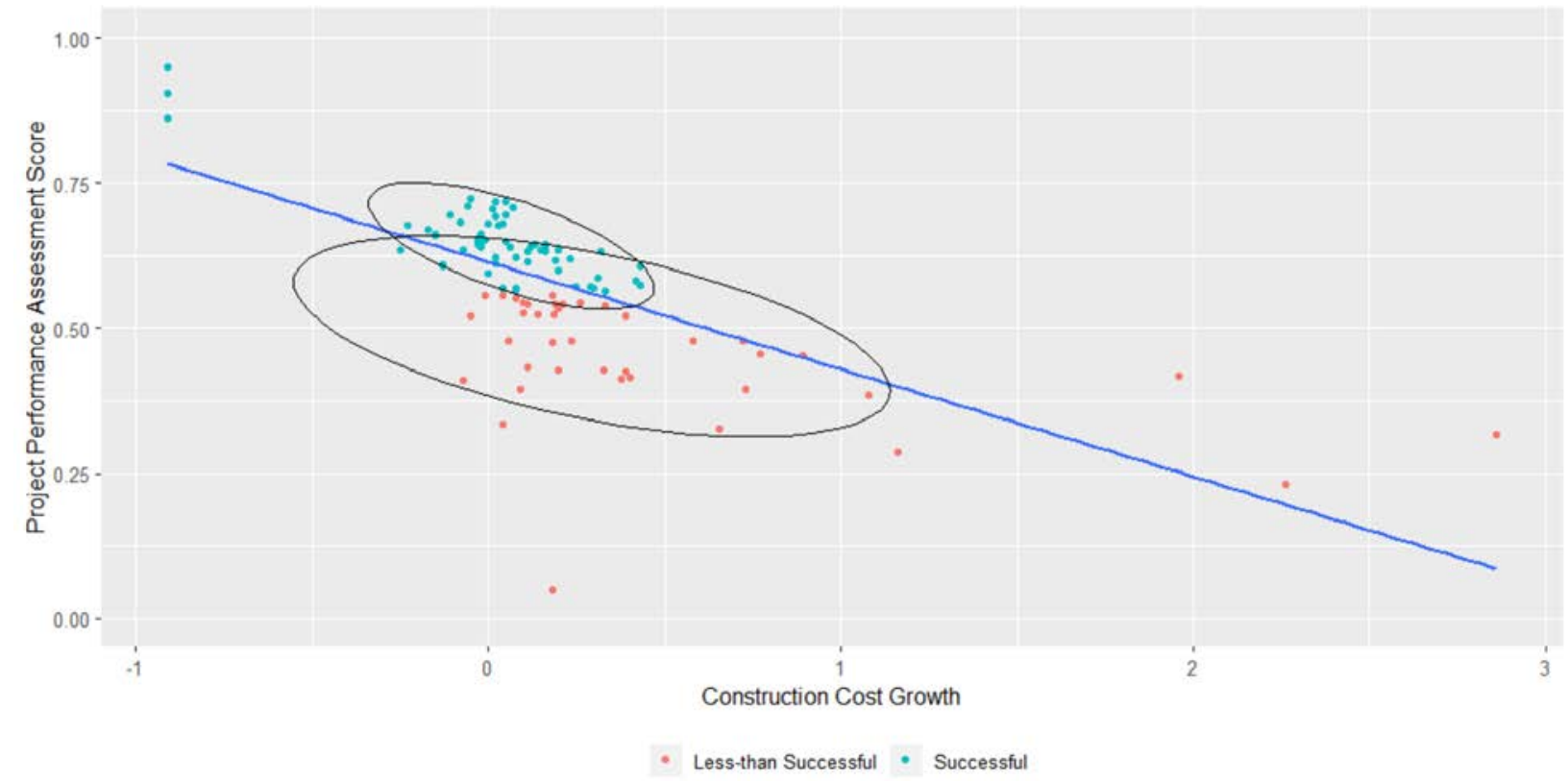

Figure 30: Plot of PPA vs construction cost growth

The differences between successful and less-than-successful projects are shown by using box-and-whisker plots. It is visually evident that successful and less-than-successful projects have similar variability to each other, as illustrated by the level of compaction of the boxplot and that was evident from the Fligner-Killeen test returning a p-value of 0.06 , confirming the equal variance of both project statuses at a 95\% confidence level. The Wilcoxon rank-sum test returned a p-value of 0.000 , which demonstrates that the data provides evidence to reject the null hypothesis at a 5\% significance level, thus concluding at a 95\% confidence level that there is statistically significant evidence that construction cost growth varies across the status of a project. Thus, it reflects the high weight that was assigned to it by the mathematical model. 


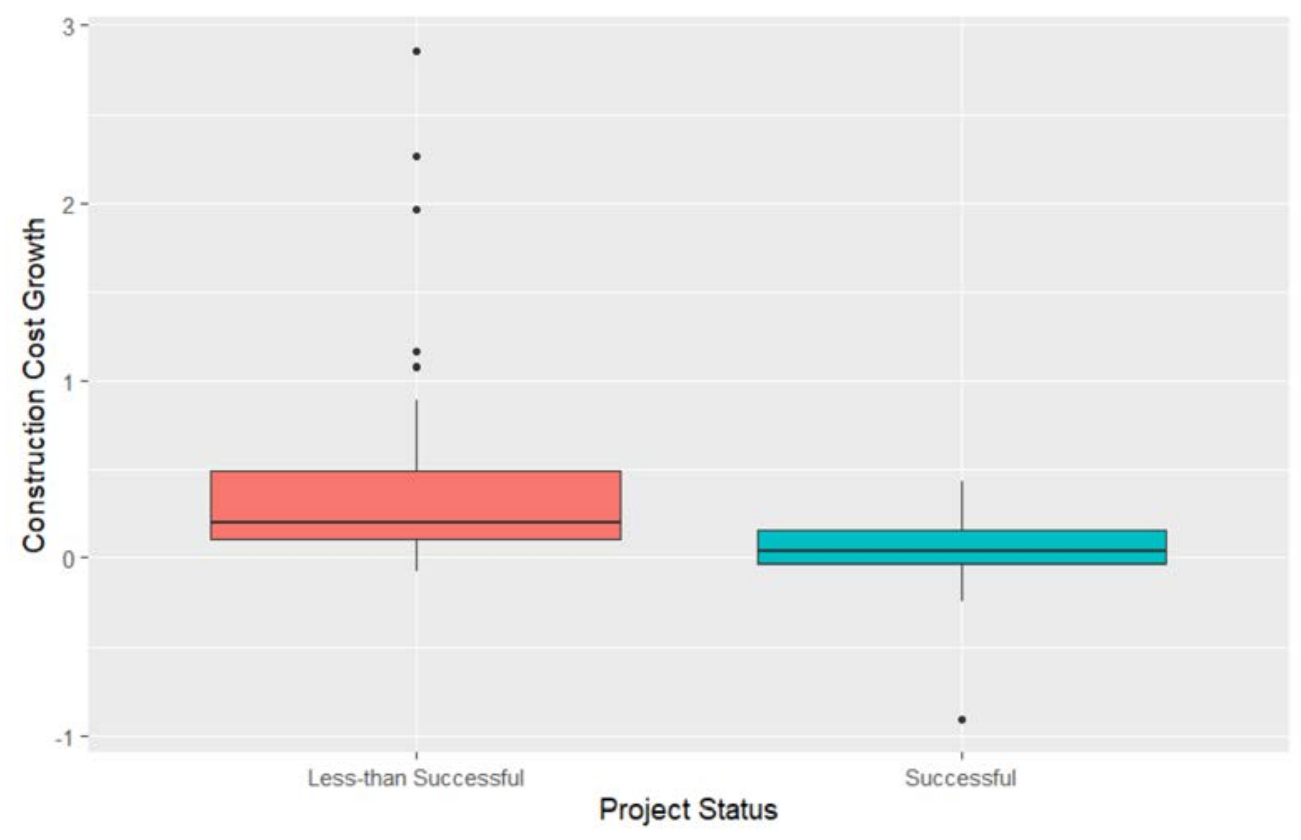

Figure 31: Boxplot of construction cost growth

The table of descriptive statistics below shows that the median/mean for construction schedule growth for successful projects (4/2.05\%) is smaller than less-than-successful projects (20/44.3\%), which shows the importance of being on budget for project success.

Table 19: Descriptive statistics for construction cost growth

\begin{tabular}{ccc}
\hline \hline Statistical Test & Successful Projects & Less-than-successful Projects \\
\hline \hline Mean & $2.05 \%$ & $44.3 \%$ \\
Maximum & $43 \%$ & $286 \%$ \\
$75 \%$ Quantile & $16 \%$ & $49 \%$ \\
50\% Quantile & $4 \%$ & $20 \%$ \\
$25 \%$ Quantile & $-3 \%$ & $10.5 \%$ \\
Minimum & $-91 \%$ & $-7.14 \%$ \\
\hline \hline
\end{tabular}




\subsubsection{Percent Delta}

A Q-Q plot and Shapiro-Wilk test are conducted to assess the normality assumption. The following Q-Q plot shows a clear departure from normality at the overall level of the dataset.
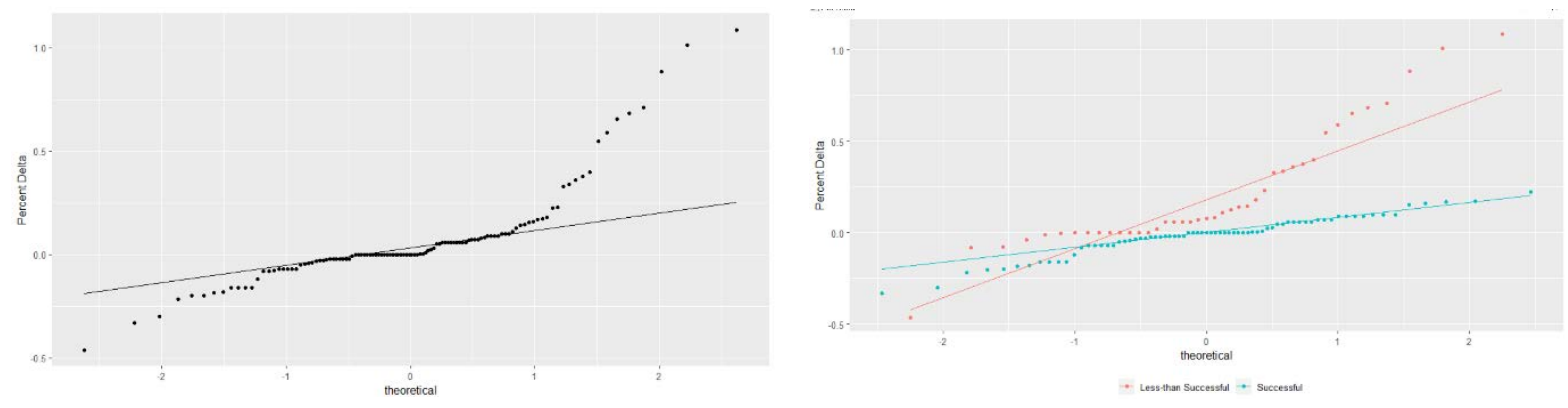

Figure 32: Q-Q p-value of percent delta

As with the other analysis, the normality assumption is not valid, as it was confirmed at a 95\% confidence level by the Shapiro-Wilk test, which is shown in the table below.

Table 20: Shapiro-Wilk Test for percent delta

Parameter

$p$-value

Significance at a 95\% Confidence Level

\begin{tabular}{ccl}
\hline \hline Overall & 0.000 & Significant \\
Successful Projects & 0.006 & Significant \\
Less-than-successful Projects & 0.000 & Significant \\
\hline \hline
\end{tabular}

The following figure shows that there is a moderate to strong negative correlation between PPA scores and the percent delta. The correlation is statistically significant at a 95\% confidence level with a p-value of 0.000 and a correlation coefficient of -0.59 . The plot chart also reveals a negative linear relationship for both successful and less-than-successful projects between PPA 
scores and the percent delta. However, it can be noticed that successful projects follow a sharper linear relationship as opposed to less-than-successful ones. For both successful and lessthan-successful projects, the correlation is statistically significant at a 95\% confidence level with a $p$-value of 0.000 for both and a correlation coefficient of -0.83 and -0.60 , respectively.

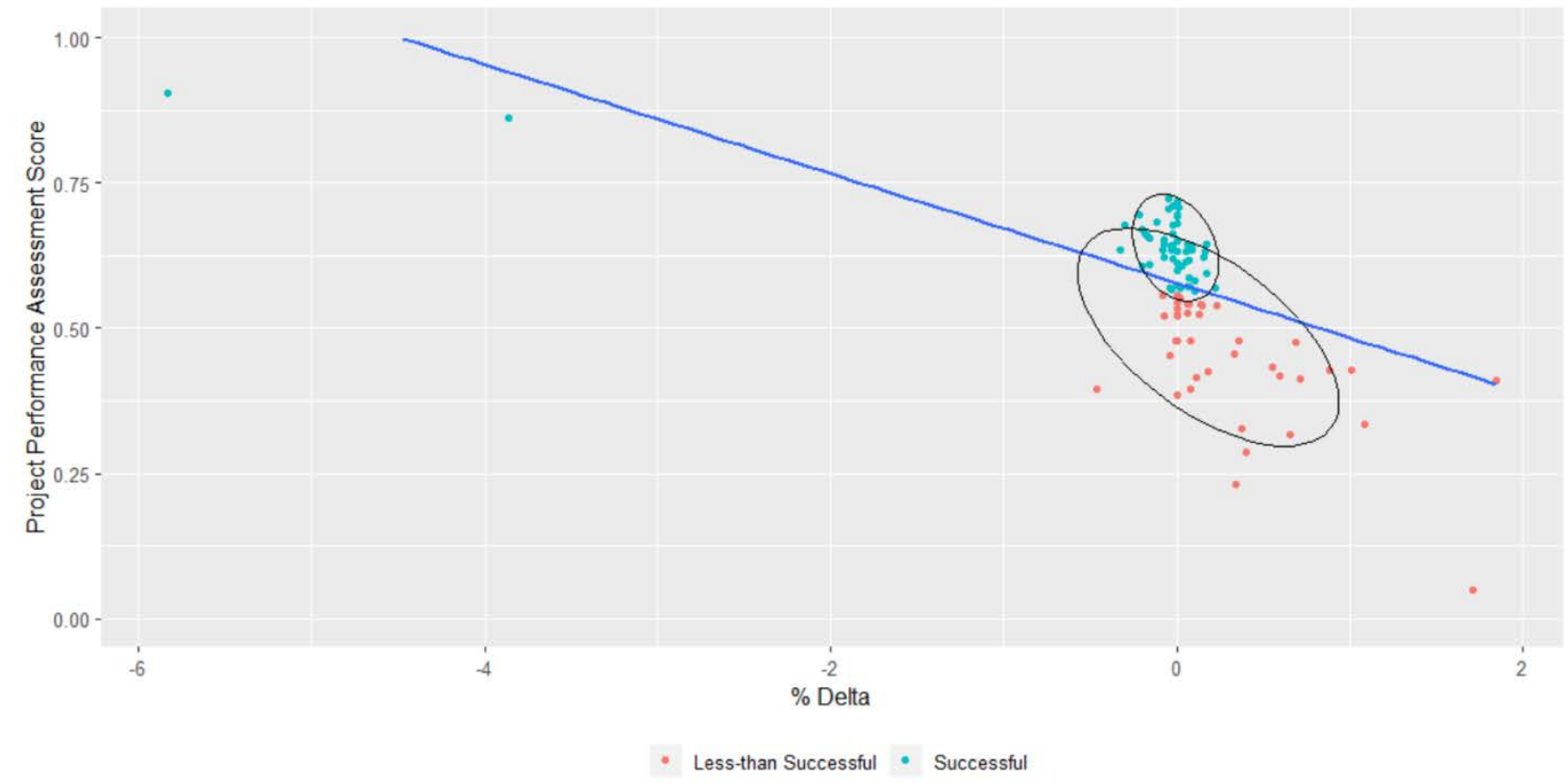

Figure 33:Plot of PPA vs percent delta

The differences between successful and less-than-successful projects are shown by using box-andwhisker plots. It is visually evident that less-than-successful projects have higher variability than successful ones, as illustrated by the level of compaction of the boxplot. That was also evident from the Fligner-Killeen test returning a p-value of 0.004 , which rejects the null hypothesis of equal variance among project status, confirming the previous suggestion of higher variability among less-than-successful projects at a 95\% confidence level. The Wilcoxon rank-sum test returned a p-value of 0.000 , which demonstrates that the data provides evidence to reject the null hypothesis at a 5\% significance level, thus concluding at a 95\% confidence level that there is 
statistically significant evidence that percent delta varies across the status of a project. Thus, it reflects the high weight that was assigned to it by the mathematical model.

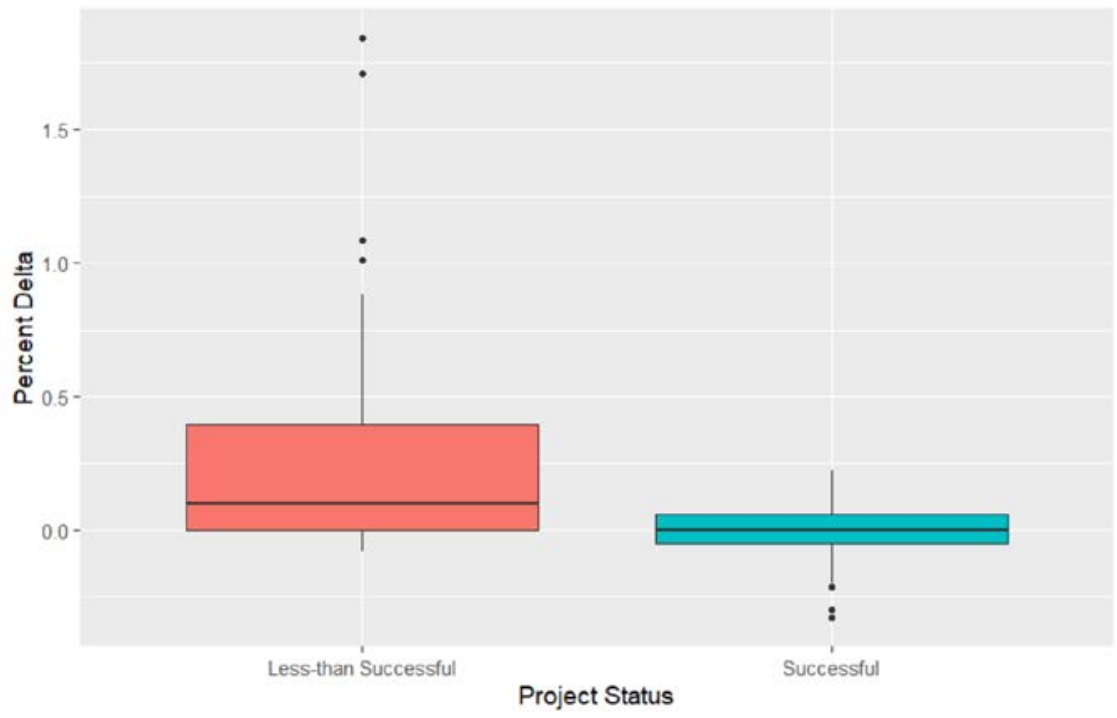

Figure 34: Boxplot of percent delta

The table of descriptive statistics below shows that the median/mean for percent delta for successful projects (0/-1.33\%) is smaller than less-than-successful projects $(9.58 / 30.3 \%)$, which shows the importance of considering the productivity factors for project success.

Table 21: Descriptive statistics for percent delta

\begin{tabular}{ccc}
\hline \hline Statistical Test & Successful Projects & Less-than-successful Projects \\
\hline \hline Mean & $-1.33 \%$ & $30.3 \%$ \\
Maximum & $22.3 \%$ & $184.6 \%$ \\
75\% Quantile & $6 \%$ & $39.4 \%$ \\
50\% Quantile & $0 \%$ & $9.58 \%$ \\
25\% Quantile & $-5 \%$ & $0 \%$ \\
Minimum & $-33 \%$ & $-8 \%$ \\
\hline \hline
\end{tabular}




\subsubsection{Construction Schedule Growth}

A Q-Q plot and Shapiro-Wilk test are conducted to assess the normality assumption. The following Q-Q plot shows a clear departure from normality at the overall level of the dataset.
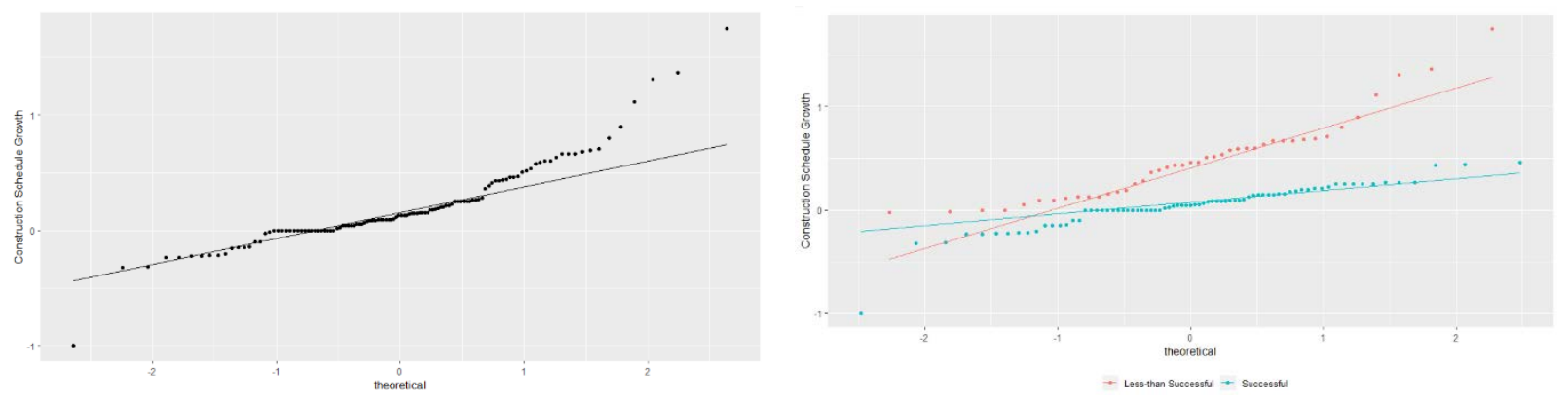

Figure 35: Q-Q plot of construction schedule growth

As with the other analysis, the normality assumption is not valid, as it was confirmed at a 95\% confidence level by the Shapiro-Wilk test, which is shown in the table below.

Table 22: Shapiro-Wilk test for construction schedule growth

Parameter

Overall

Successful Projects

Less-than-successful Projects $p$-value

0.000

0.000

0.002
Significance at a 95\% Confidence Level
Significant

Significant

Significant

The following figure shows that there is a moderate to strong negative correlation between PPA scores and the construction schedule growth. The correlation is statistically significant at a 95\% confidence level with a p-value of 0.000 and a correlation coefficient of -0.72 . The plot chart also reveals a negative linear relationship for both successful and less-than-successful projects between PPA scores and the construction schedule growth. However, it can be noticed that less- 
than-successful projects follow a sharper linear relationship relative to the successful ones. For both successful and less-than-successful projects, the correlation is statistically significant at a 95\% confidence level with a $p$-value of 0.000 for both and a correlation coefficient of -0.43 and 0.59 , respectively.

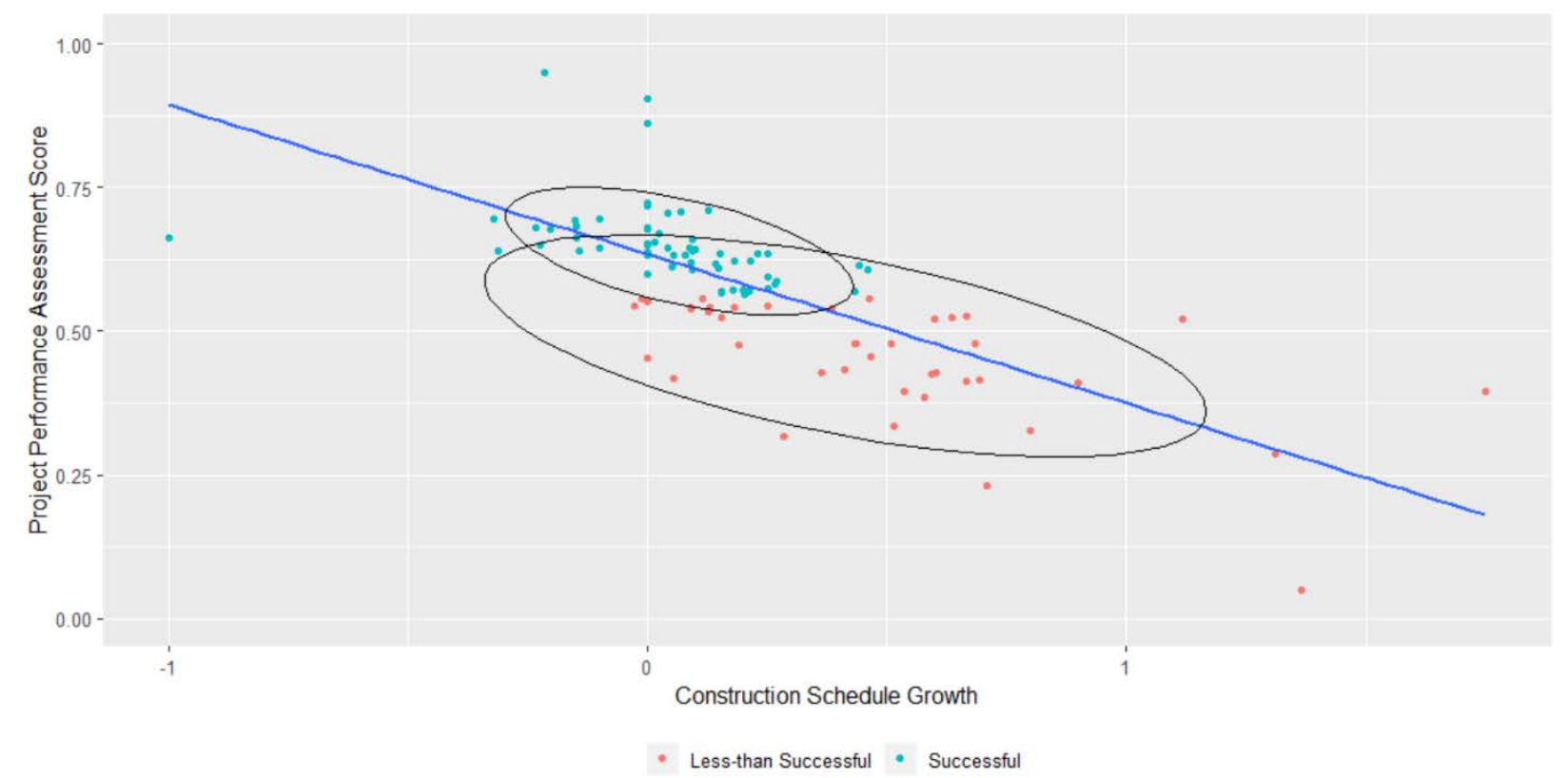

Figure 36: Plot of PPA vs construction schedule growth

The differences between successful and less-than-successful projects are shown by using box-and-whisker plots. It is visually evident that less-than-successful projects have higher variability than successful ones, as illustrated by the level of compaction of the boxplot. That was also evident from the Fligner-Killeen test returning a p-value of 0.000 , which rejects the null hypothesis of equal variance among project status, confirming the previous suggestion of higher variability among less-than-successful projects at a 95\% confidence level. The Wilcoxon ranksum test returned a p-value of 0.000 , which demonstrates that the data provides evidence to reject the null hypothesis at a 5\% significance level, thus concluding at a 95\% confidence level that there 
is statistically significant evidence that construction schedule growth varies across the status of a project. Thus, it reflects the high weight that was assigned to it by the mathematical model.

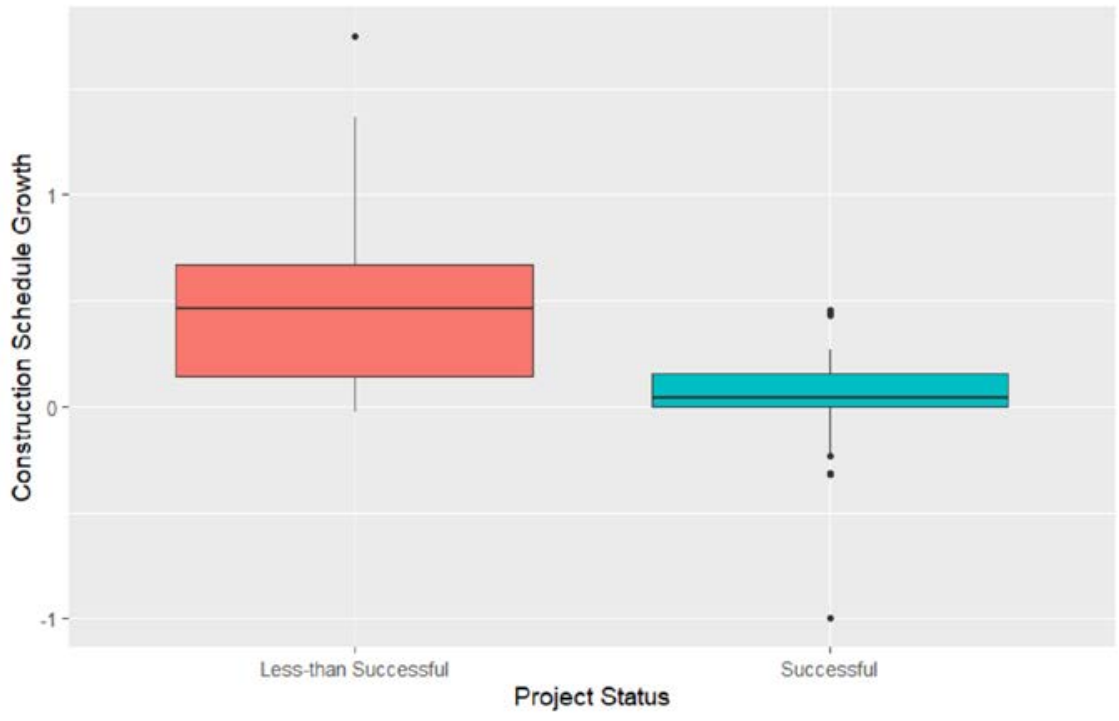

Figure 37: Boxplot of construction schedule growth

The table of descriptive statistics below shows that the median/mean for construction schedule growth for successful projects $(4.27 / 4.17 \%)$ is smaller than less-than-successful projects (46.3/47.9\%), which shows the importance of being on time for project success.

Table 23: Descriptive statistics for construction schedule growth

\begin{tabular}{ccc}
\hline \hline Statistical Test & Successful Projects & Less-than-successful Projects \\
\hline \hline Mean & $4.17 \%$ & $47.9 \%$ \\
Maximum & $45.9 \%$ & $175 \%$ \\
$75 \%$ Quantile & $15.4 \%$ & $66.7 \%$ \\
$50 \%$ Quantile & $4.27 \%$ & $46.3 \%$ \\
$25 \%$ Quantile & $0 \%$ & $14.2 \%$ \\
Minimum & $-100 \%$ & $-2.7 \%$ \\
\hline \hline
\end{tabular}




\subsubsection{Lost-Time Injuries per Millions of Dollars}

A Q-Q plot and Shapiro-Wilk test are conducted to assess the normality assumption. The following Q-Q plot shows a clear departure from normality at the overall level of the dataset.
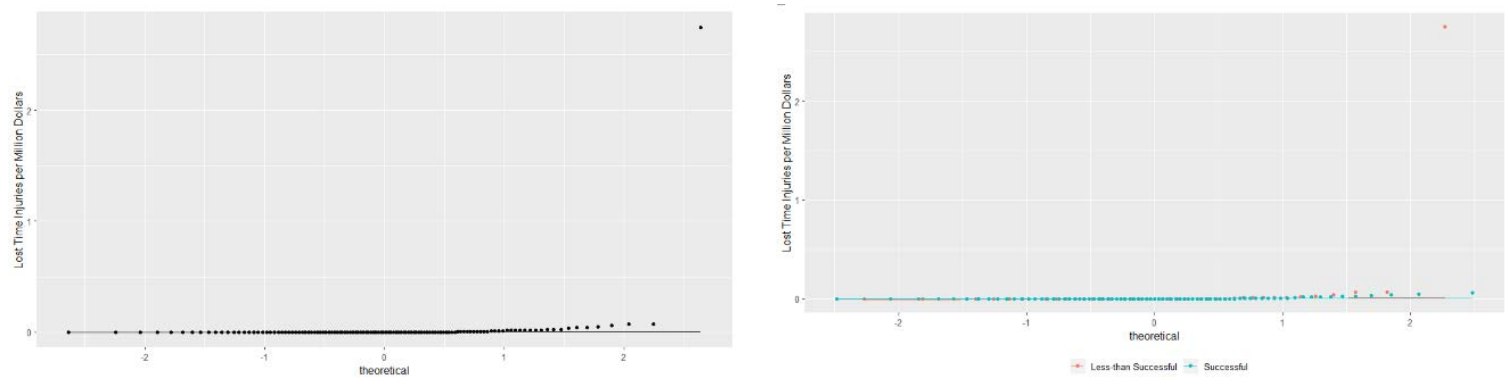

Figure 38: Q-Q plot of lost-time injuries per millions of dollars

As with the other analysis, the normality assumption is not valid, as it was confirmed at a 95\% confidence level by the Shapiro-Wilk test, which is shown in the table below.

Table 24: Shapiro-Wilk test for lost-time injuries per millions of dollars

\begin{tabular}{ccc}
\hline \hline Parameter & $p$-value & Significance at a 95\% Confidence Level \\
\hline \hline Overall & 0.000 & Significant \\
Successful Projects & 0.000 & Significant \\
Less-than-successful Projects & 0.000 & Significant \\
\hline \hline
\end{tabular}

The following figure shows that there is a weak to moderate negative correlation between PPA scores and the lost-time injuries per millions of dollars. The correlation is statistically significant at a 95\% confidence level with a p-value of 0.043 and a correlation coefficient of -0.19 . However, the plot chart reveals a very weak negative linear relationship for both successful and less-than-successful projects between PPA scores and the lost-time injuries per millions of dollars. 
This is evident from the correlation test, which shows that it is not statistically significant at a 95\% confidence level with a p-value of 0.065 and 0.241 , and a correlation coefficient of -0.21 and -0.18 respectively.

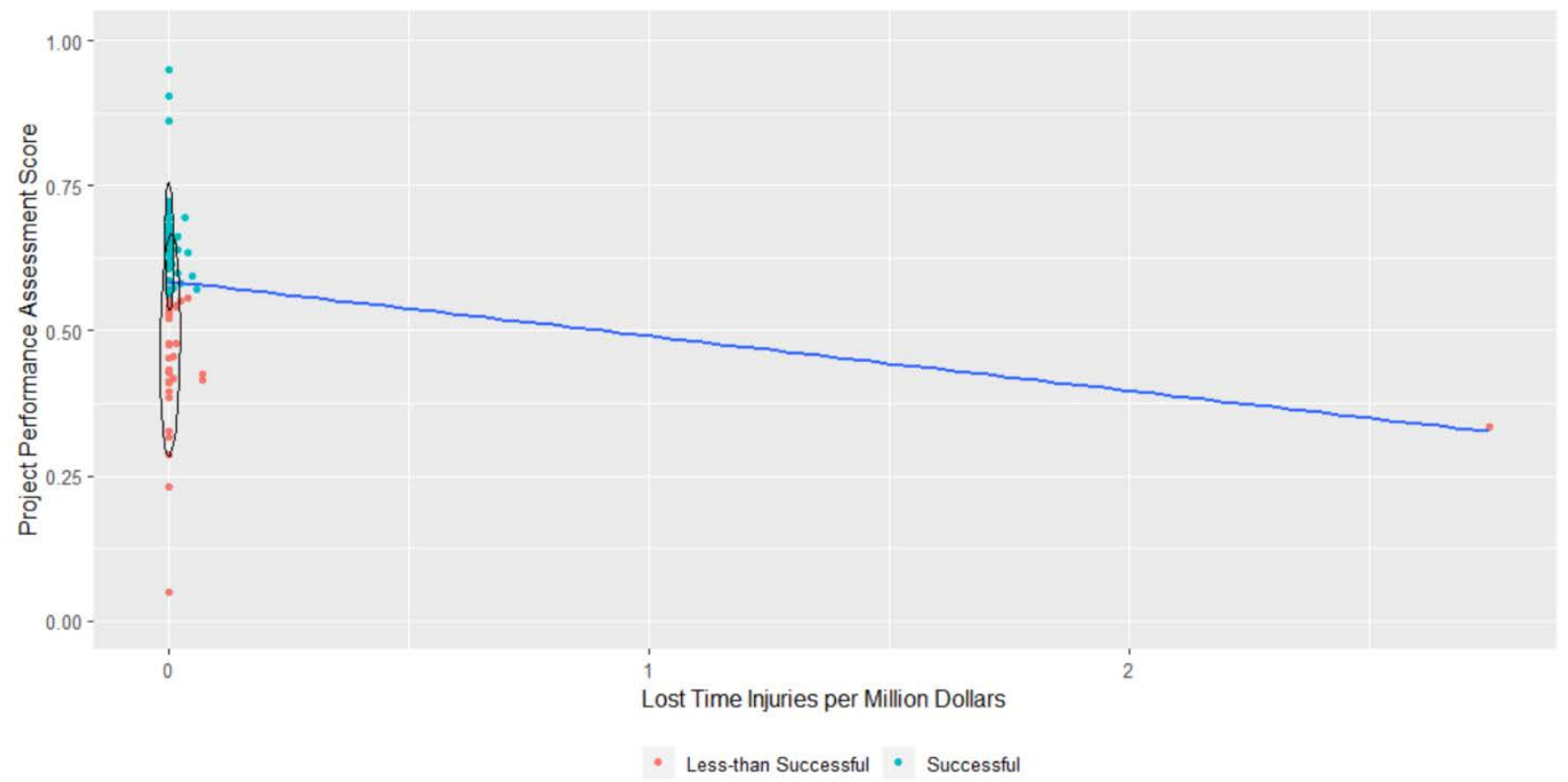

Figure 39: Plot of PPA vs lost-time injuries per millions of dollars

The differences between successful and less-than-successful projects are shown by using box-and-whisker plots. It is visually evident that successful and less-than-successful projects have similar variability to each other, as illustrated by the level of compaction of the boxplot and that was evident from the Fligner-Killeen test returning a p-value of 0.554, confirming the equal variance of both project statuses at a 95\% confidence level. The Wilcoxon rank-sum test returned a p-value of 0.838 , which demonstrates that the data does not provide enough evidence to reject the null hypothesis at a 5\% significance level, thus concluding at a 95\% confidence level that there is no statistically significant evidence that lost-time injuries per millions of dollars vary across the status of a project. Thus, it reflects the small weight that was assigned to it by the mathematical model. 


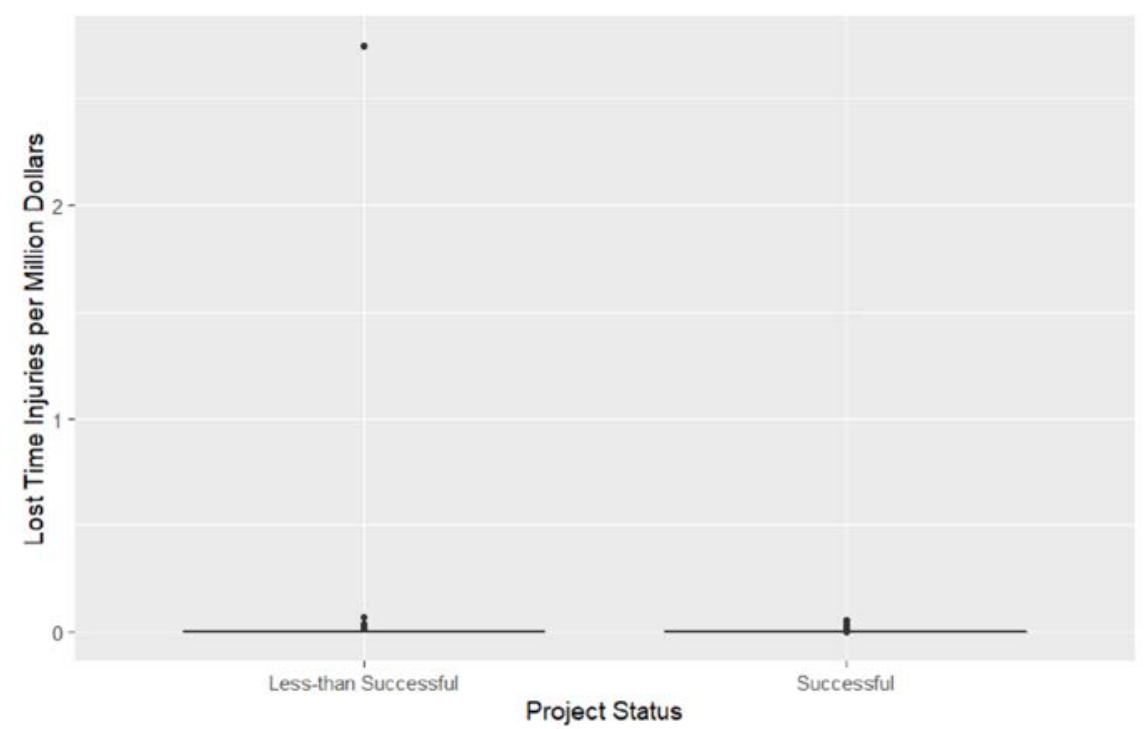

Figure 40: Boxplot of lost-time injuries per millions of dollars

The table of descriptive statistics below shows that the median for the lost-time injuries per millions of dollars is equal to 0 for both successful and less-than-successful projects. However, the mean for the lost time injuries per millions of dollars for successful projects $(0.0044)$ is slightly smaller than less-than-successful projects (0.071), which again shows the importance of having safety measures to avoid injuries and fatalities incidents.

Table 25: Descriptive statistics for lost-time injuries per millions of dollars

\begin{tabular}{ccc}
\hline \hline Statistical Test & Successful Projects & Less-than-successful Projects \\
\hline \hline Mean & 0.0044 & 0.071 \\
Maximum & 0.0571 & 2.75 \\
$75 \%$ Quantile & 0.0015 & 0.00558 \\
50\% Quantile & 0 & 0 \\
25\% Quantile & 0 & 0 \\
Minimum & 0 & 0 \\
\hline \hline
\end{tabular}




\subsubsection{Rework Percentage}

A Q-Q plot and Shapiro-Wilk test are conducted to assess the normality assumption. The following Q-Q plot shows a clear departure from normality at the overall level of the dataset.
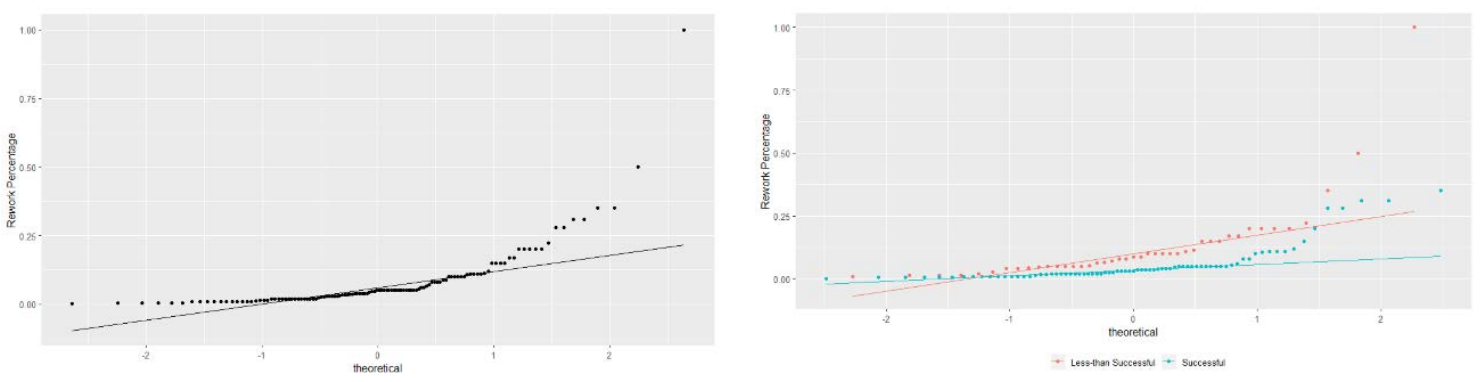

Figure 41: Q-Q plot of rework percentage

As with the other analysis, the normality assumption is not valid, as it was confirmed at a 95\% confidence level by the Shapiro-Wilk test, which is shown in the table below.

Table 26: Shapiro-Wilk test for rework percentage

\begin{tabular}{ccc}
\hline \hline Parameter & $p$-value & Significance at a 95\% Confidence Level \\
\hline Overall & 0.000 & Significant \\
Successful Projects & 0.000 & Significant \\
Less-than-successful Projects & 0.000 & Significant \\
\hline \hline
\end{tabular}

The following figure shows a weak to moderate negative correlation between PPA scores and the rework percentage. The correlation is statistically significant at a 95\% confidence level with a p-value of 0.012 and a correlation coefficient of -0.23 . However, the plot chart reveals a weak to moderate positive linear relationship for successful projects between PPA scores and the rework percentage, in which the correlation is statistically significant at a 95\% confidence level 
with a p-value of 0.004 and a correlation coefficient of 0.24 . While there is a weak to moderate negative linear relationship for less-than-successful projects between PPA scores and the rework percentage, it is evident that the correlation test is not statistically significant at a 95\% confidence level with a $p$-value of 0.225 and a correlation coefficient of -0.19 .

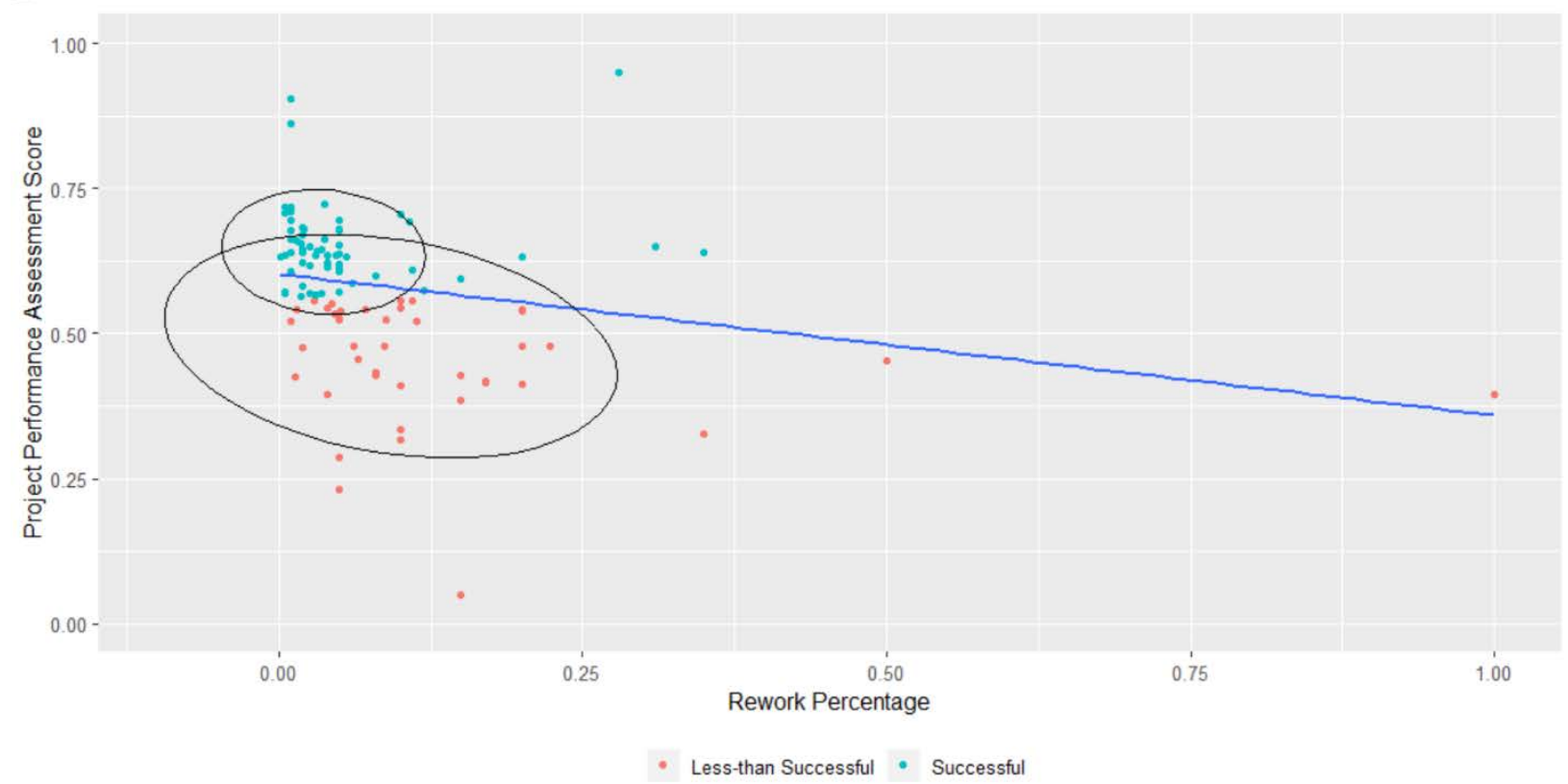

Figure 42: Plot of PPA vs rework percentage

The differences between successful and less-than-successful projects are shown by using box-and-whisker plots. It is visually evident that less-than-successful projects have higher variability than successful ones, as illustrated by the level of compaction of the boxplot. That was also evident from the Fligner-Killeen test returning a p-value of 0.001 , which rejects the null hypothesis of equal variance among project status, confirming the previous suggestion of higher variability among less-than-successful projects at a 95\% confidence level. The Wilcoxon ranksum test returned a p-value of 0.000 , which demonstrates that the data provides evidence to reject the null hypothesis at a 5\% significance level, thus concluding at a 95\% confidence level that there 
is statistically significant evidence that the rework percentage varies across the status of a project. Thus, it reflects the importance of using this performance metric in the mathematical model.

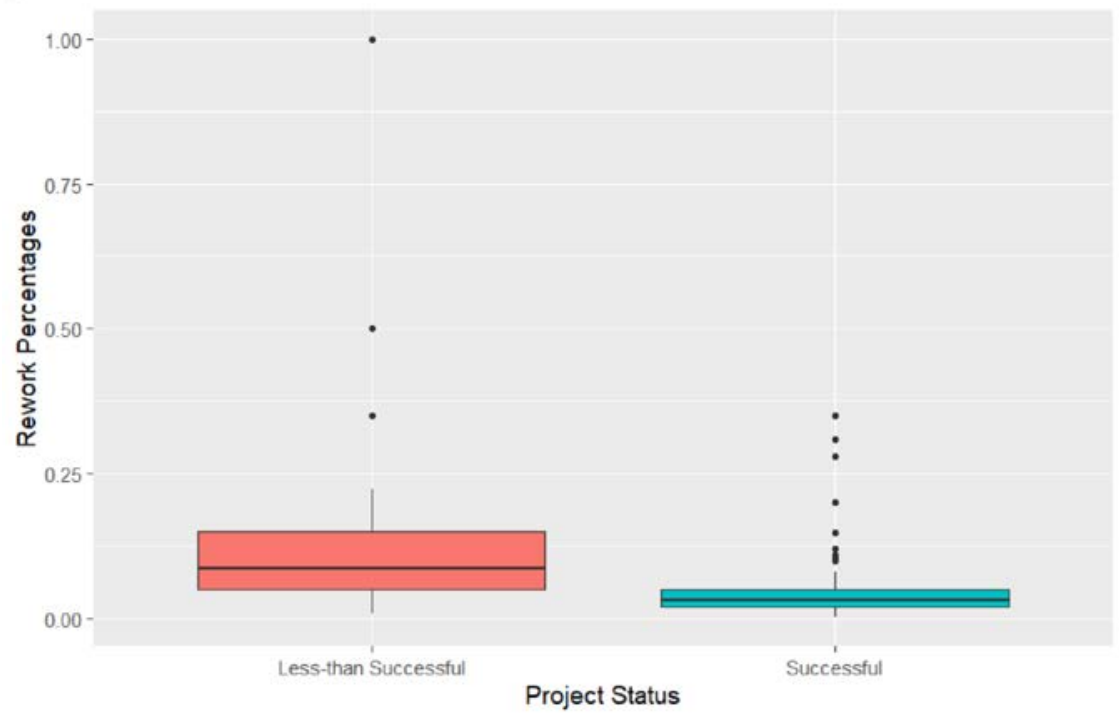

Figure 43: Boxplot of rework percentage

The table of descriptive statistics below shows that the median/mean for rework percentage for successful projects (3.1/5.7\%) is smaller than less-than-successful projects (8.72/12.8\%), which shows the importance of having good quality control measures to avoid rework percentage.

Table 27: Descriptive statistics for rework percentage

\begin{tabular}{ccc}
\hline \hline Statistical Test & Successful Projects & Less-than-successful Projects \\
\hline \hline Mean & $5.7 \%$ & $12.8 \%$ \\
Maximum & $35 \%$ & $100 \%$ \\
$75 \%$ Quantile & $5 \%$ & $15 \%$ \\
$50 \%$ Quantile & $3.1 \%$ & $8.72 \%$ \\
$25 \%$ Quantile & $2 \%$ & $5 \%$ \\
Minimum & $0.18 \%$ & $1 \%$ \\
\hline \hline
\end{tabular}




\subsubsection{Value of Punch List Items}

A Q-Q plot and Shapiro-Wilk test are conducted to assess the normality assumption. The following Q-Q plot shows a clear departure from normality at the overall level of the dataset.
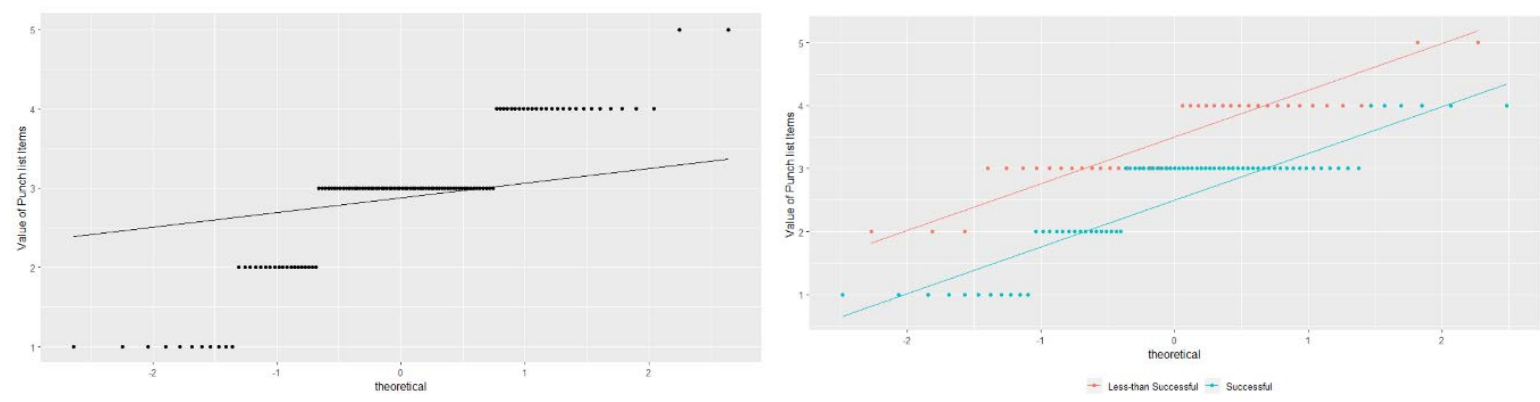

Figure 44: Q-Q plot of value of punch list items

As with the other analysis, the normality assumption is not valid, as it was confirmed at a 95\% confidence level by the Shapiro-Wilk test, which is shown in the table below.

Table 28: Shapiro-Wilk test for value of punch list items

\begin{tabular}{ccc}
\hline \hline Parameter & $p$-value & Significance at a 95\% Confidence Level \\
\hline \hline Overall & 0.000 & Significant \\
Successful Projects & 0.000 & Significant \\
Less-than-Successful Projects & 0.000 & Significant \\
\hline
\end{tabular}

The following figure shows that there is a moderate negative correlation between PPA scores and the value of punch list items. The correlation is statistically significant at a $95 \%$ confidence level with a p-value of 0.000 and a correlation coefficient of -0.50 . However, the plot chart reveals a weak to moderate negative linear relationship for successful and less-than- 
successful projects between PPA scores and the value of punch list items. For successful projects, the correlation is statistically significant at a 95\% confidence level with a p-value of 0.013 and a correlation coefficient of -0.28 . While for less-than-successful projects, the correlation is not statistically significant at a 95\% confidence level with a $p$-value of 0.195 and a correlation coefficient of -0.20 .

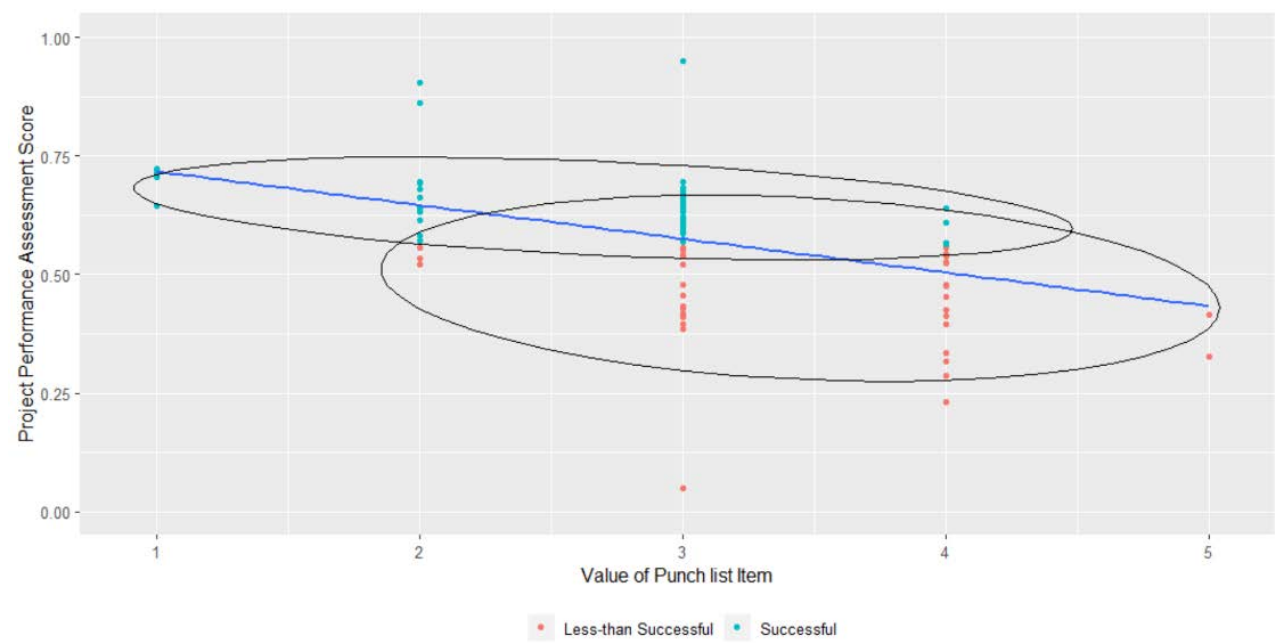

Figure 45: Plot of PPA vs value of punch list items

The differences between successful and less-than-successful projects are shown by using box-and-whisker plots. It is visually evident that less-than-successful projects have higher variability than successful ones, as illustrated by the level of compaction of the boxplot. That was also evident from the Fligner-Killeen test returning a p-value of 0.001 , which rejects the null hypothesis of equal variance among project status, confirming the previous suggestion of higher variability among less-than-successful projects at a 95\% confidence level. The Wilcoxon ranksum test returned a p-value of 0.000 , which demonstrates that the data provides evidence to reject the null hypothesis at a 5\% significance level, thus concluding at a 95\% confidence level that there is statistically significant evidence that value of punch list items varies across the status of a 
project. Thus, it reflects the importance of using this performance metric in the mathematical model.

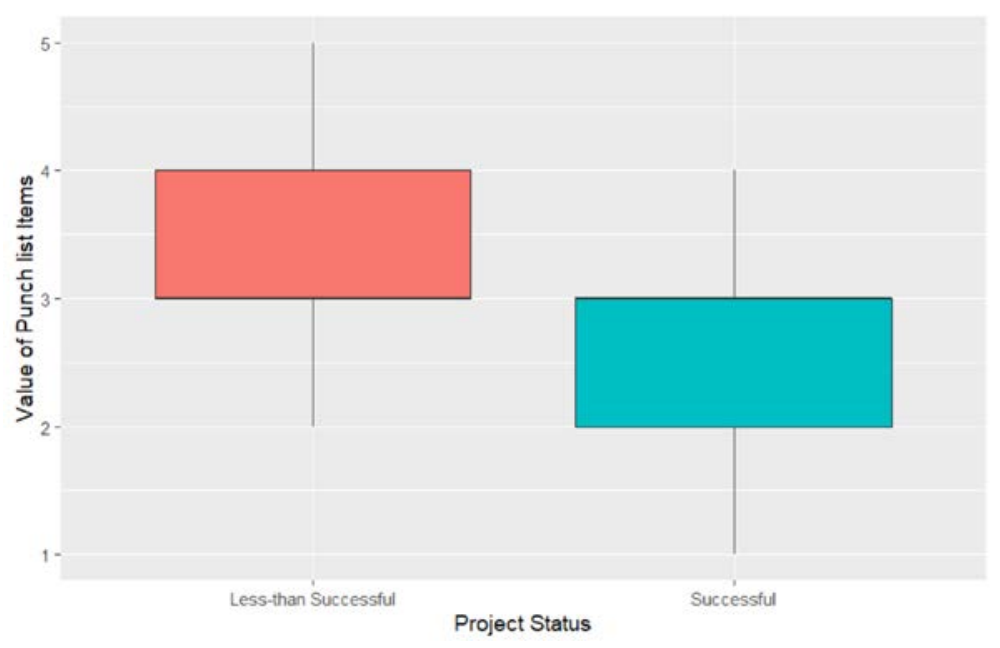

Figure 46: Boxplot of value of punch list items

The table of descriptive statistics below shows that the median for the value of punch list items is equal to 3 for both successful and less-than-successful projects. However, the mean for the value of punch list items for successful projects (2.58) is slightly smaller than less-thansuccessful projects (3.47), which again shows the importance of having good quality control measures to avoid the value of punch list.

Table 29: Descriptive statistics for value of punch list items

\begin{tabular}{ccc}
\hline \hline Statistical Test & Successful Projects & Less-than-successful Projects \\
\hline \hline Mean & 2.58 & 3.47 \\
Maximum & 4 & 5 \\
$75 \%$ Quantile & 3 & 4 \\
$50 \%$ Quantile & 3 & 3 \\
$25 \%$ Quantile & 2 & 3 \\
Minimum & 1 & 2 \\
\hline \hline
\end{tabular}




\subsubsection{Number of RFIs per Million Dollars}

A Q-Q plot and Shapiro-Wilk test are conducted to assess the normality assumption. The following Q-Q plot shows a clear departure from normality at the overall level of the dataset.
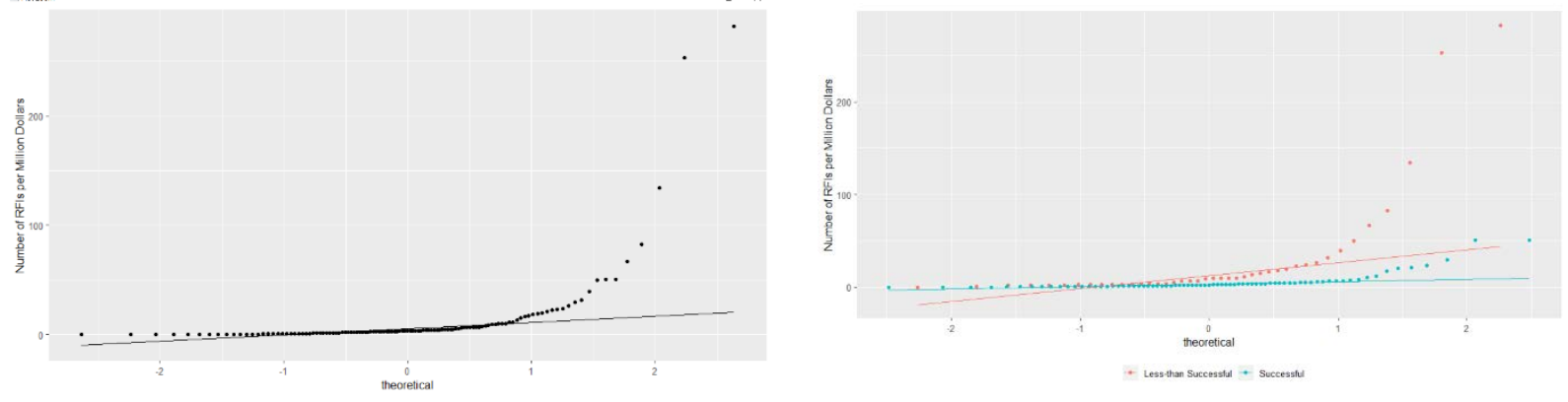

Figure 47: Q-Q Plot of number of RFIs per million dollars

As with the other analysis, the normality assumption is not valid, as it was confirmed at a 95\% confidence level by the Shapiro-Wilk test, which is shown in the table below.

Table 30: Shapiro-Wilk test for number of RFIs per million dollars

\begin{tabular}{ccc}
\hline \hline Parameter & $p$-value & Significance at a 95\% Confidence Level \\
\hline \hline Overall & 0.000 & Significant \\
Successful Projects & 0.000 & Significant \\
Less-than-successful Projects & 0.000 & Significant \\
\hline \hline
\end{tabular}

The following figure shows that there is a moderate negative correlation between PPA scores and the number of RFIs per million dollars. The correlation is statistically significant at a 95\% confidence level with a p-value of 0.000 and a correlation coefficient of -0.45 . However, the plot chart reveals a moderate negative linear relationship for successful and less-than-successful projects between PPA scores and the number of RFIs per million dollars. For successful projects, 
the correlation is statistically significant at a $95 \%$ confidence level with a p-value of 0.000 and a correlation coefficient of -0.59 . For less-than-successful projects the correlation is statistically significant at a $95 \%$ confidence level with a $p$-value of 0.000 and a correlation coefficient of -0.67 .

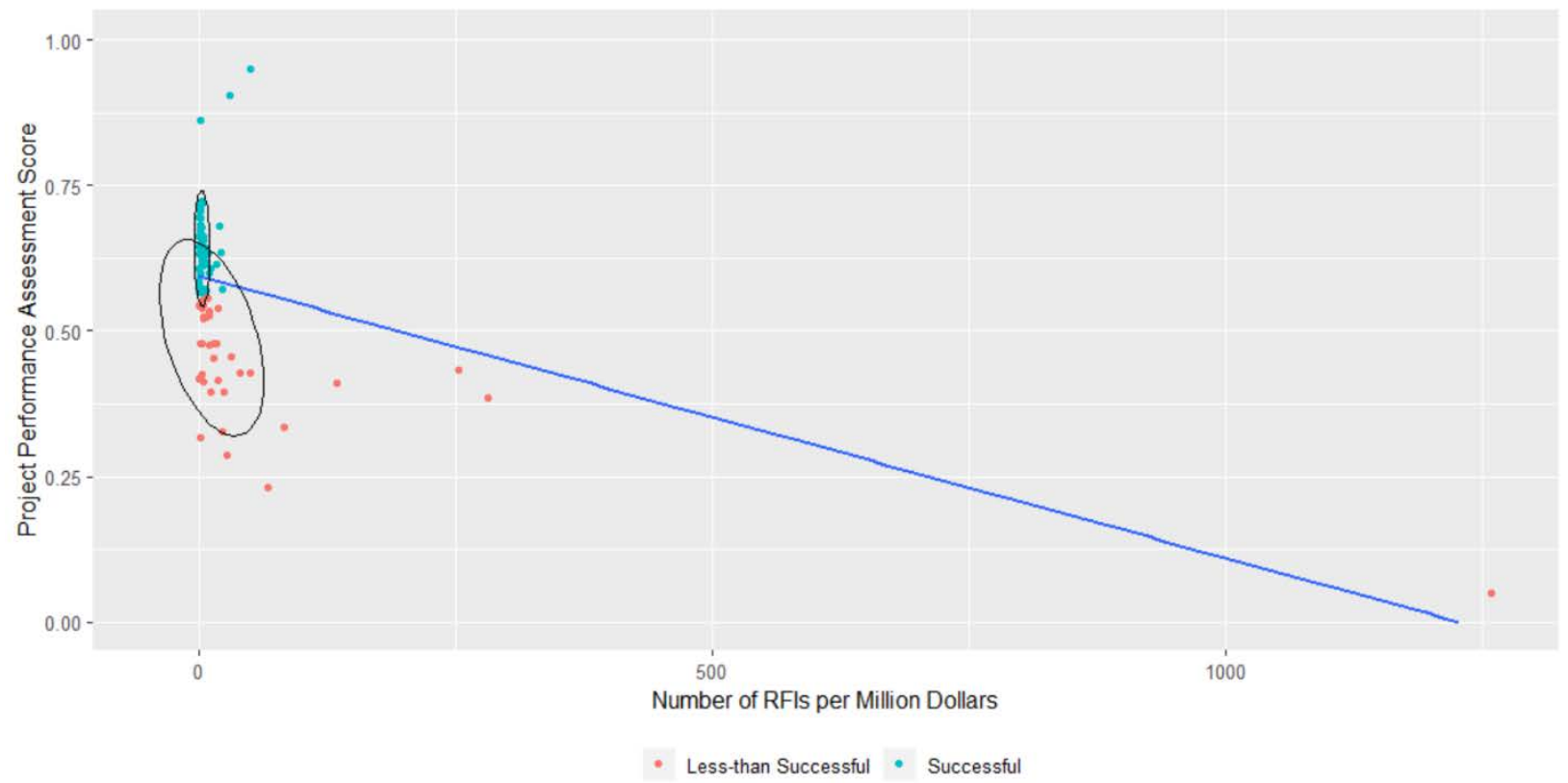

Figure 48: Plot of PPA vs number of RFIs per million dollars

The differences between successful and less-than-successful projects are shown by using box-and-whisker plots. It is visually evident that less-than-successful projects have a higher variability than successful ones, as illustrated by the level of compaction of the boxplot. That was also evident from the Fligner-Killeen test returning a p-value of 0.000 , which rejects the null hypothesis of equal variance among project status, confirming the previous suggestion of a higher variability among less-than-successful projects at a 95\% confidence level. The Wilcoxon ranksum test returned a p-value of 0.000 , which demonstrates that the data provides evidence to reject the null hypothesis at a 5\% significance level, thus concluding at a 95\% confidence level that there is statistically significant evidence that the number of RFIs per million dollars varies across the 
status of a project. Thus, it reflects the importance of using this performance metric in the mathematical model.

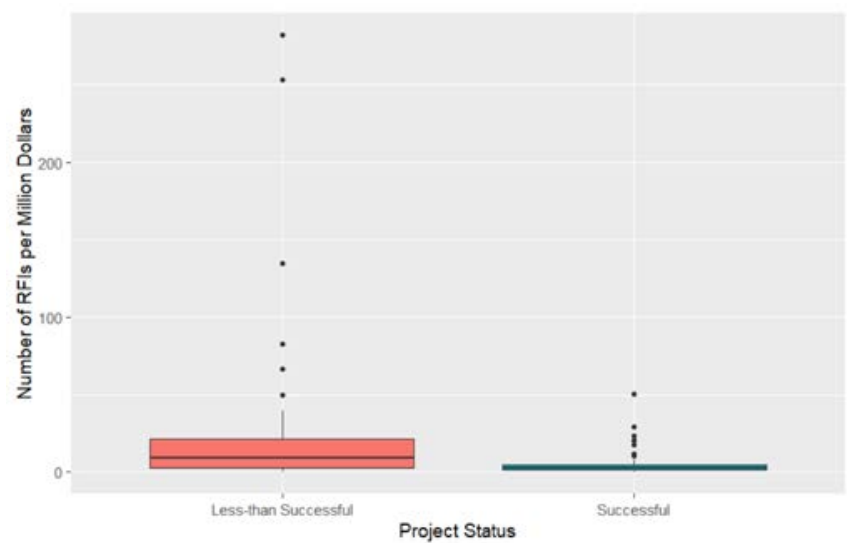

Figure 49: Boxplot of number of RFIs per million dollars

The table of descriptive statistics below shows that the median for number of the RFIs per million dollars for less-than-successful projects (9.56) is approximately 3.75x that of the successful projects (2.54). The mean for the number of RFIs per million dollars for less-than-successful projects (57.37) is greater than 10x that of the successful projects (57.73). These results further show the importance of having strong and clear communication for project success.

Table 31: Descriptive statistics for number of RFIs per million dollars

\begin{tabular}{ccc}
\hline \hline Statistical Test & Successful Projects & Less-than-successful Projects \\
\hline \hline Mean & 5.37 & 57.73 \\
Maximum & 50.74 & 1259.4 \\
75\% Quantile & 4.75 & 23.4 \\
50\% Quantile & 2.54 & 9.56 \\
25\% Quantile & 1.23 & 3.18 \\
Minimum & 0.11 & 0.09 \\
\hline \hline
\end{tabular}




\subsubsection{RFI Processing Time}

A Q-Q plot and Shapiro-Wilk test are conducted to assess the normality assumption. The following Q-Q plot shows a clear departure from normality at the overall level of the dataset.
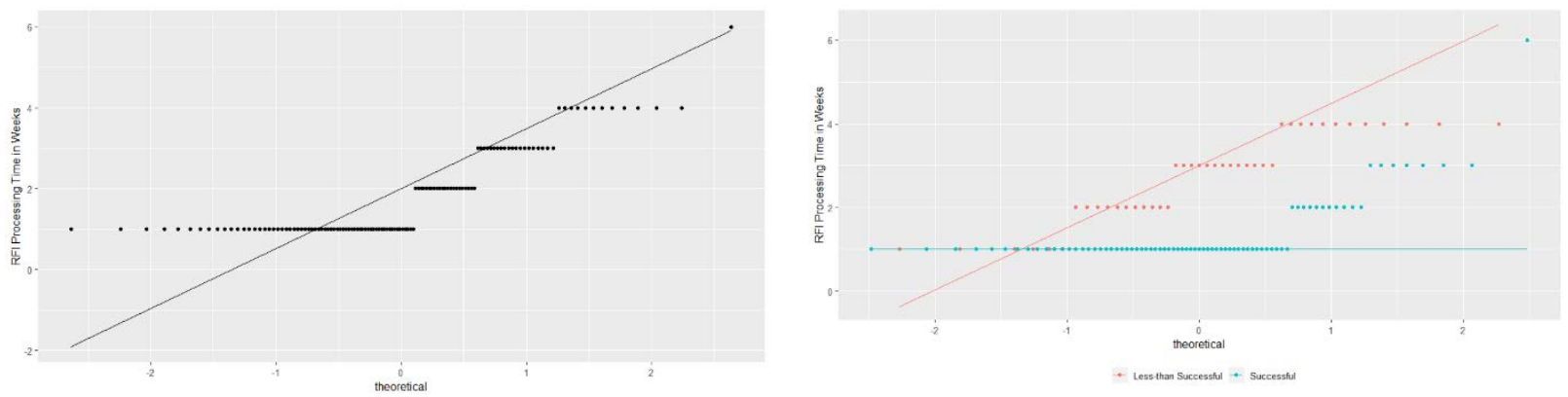

Figure 50: Q-Q plot of RFI processing time

As with the other analysis, the normality assumption is not valid, as confirmed by a 95\% confidence level by the Shapiro-Wilk test, which is shown in the table below.

Table 32: Shapiro-Wilk test for RFI processing time

\begin{tabular}{ccc}
\hline \hline Parameter & $p$-value & Significance at a 95\% Confidence Level \\
\hline Overall & 0.000 & Significant \\
Successful Projects & 0.000 & Significant \\
Less-than-successful Projects & 0.000 & Significant \\
\hline \hline
\end{tabular}

The following figure shows that there is a moderate negative correlation between PPA scores and the RFI processing time. The correlation is statistically significant at a $95 \%$ confidence level with a p-value of 0.000 and a correlation coefficient of -0.53 . However, the plot chart reveals a weak to moderate negative linear relationship for successful and less-than-successful projects between PPA scores and the RFI processing time. For successful projects, the correlation is not 
statistically significant at a $95 \%$ confidence level with a p-value of 0.058 and a correlation coefficient of -0.22 . For less-than-successful projects, the correlation is also not statistically significant at a 95\% confidence level with a $p$-value of 0.262 and a correlation coefficient of -0.17 .

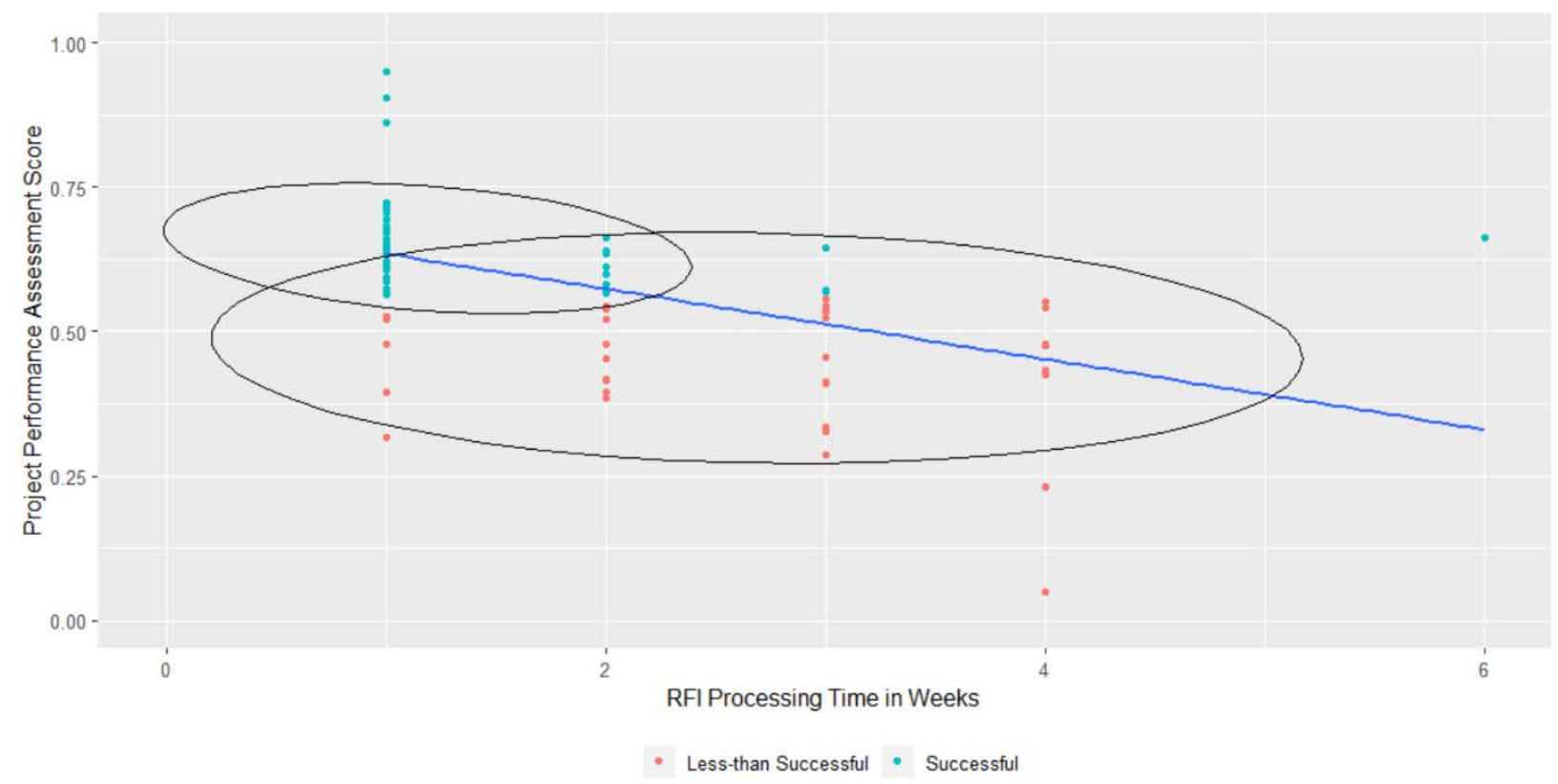

Figure 51: Plot of PPA vs RFI processing time

The differences between successful and less-than-successful projects are shown by using box-and-whisker plots. By this, it is visually evident that less-than-successful projects have higher variability than successful ones, as illustrated by the level of compaction of the boxplot. This was further evident from the Fligner-Killeen test returning a p-value of 0.000, which rejects the null hypothesis of equal variances among project status, confirming the previous suggestion of higher variability among less-than-successful projects at a 95\% confidence level. The Wilcoxon ranksum test returned a p-value of 0.000 , which demonstrates that the data provides evidence to reject the null hypothesis at a 5\% significance level, thus concluding at the $95 \%$ confidence level that there is statistically significant evidence that the RFI processing time varies across the status of a 
project. Thus, it reflects the importance of using this performance metric in the mathematical model.

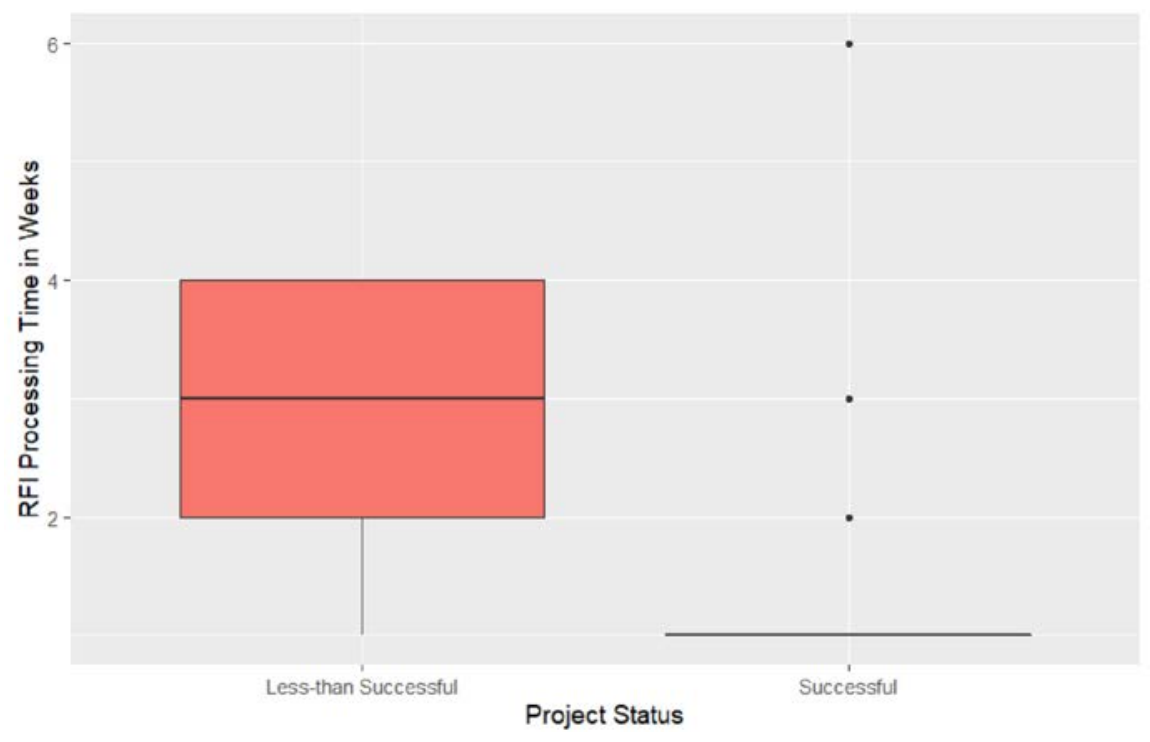

Figure 52: Boxplot of RFI processing time

The table of descriptive statistics below shows that the median/mean for RFI processing time for successful projects (1/1.39) is smaller than the less-than-successful projects $(3 / 2.70)$. These results show the importance of having clear and strong communication for project success.

Table 33: Descriptive statistics for RFI processing time

\begin{tabular}{ccc}
\hline \hline Statistical Test & Successful Projects & Less-Than-Successful Projects \\
\hline \hline Mean & 1.39 & 2.70 \\
Maximum & 6 & 4 \\
$75 \%$ Quantile & 1 & 4 \\
$50 \%$ Quantile & 1 & 3 \\
$25 \%$ Quantile & 1 & 2 \\
Minimum & 1 & 1 \\
\hline \hline
\end{tabular}




\section{6. $\quad$ PPA Model Robustness}

Model robustness is performed for validating the PPA model to check that the results are not highly determined by changes in the dataset. In this method, the entire dataset is randomly split into five parts, where four parts of the dataset are used to replicate the results obtained by the PPA mathematical model. This process is repeated five times. Figure 52 shows the maximum and minimum deviations for the weights of the eight performance metrics. The mean absolute percent error (MAPE) for the model is computed by calculating the average value of the absolute percent errors of the eight performance metrics in the five trials, which add up to 40 data points. The PPA model has a MAPE of 7.0\%, which is reasonable for the type of data analyzed.

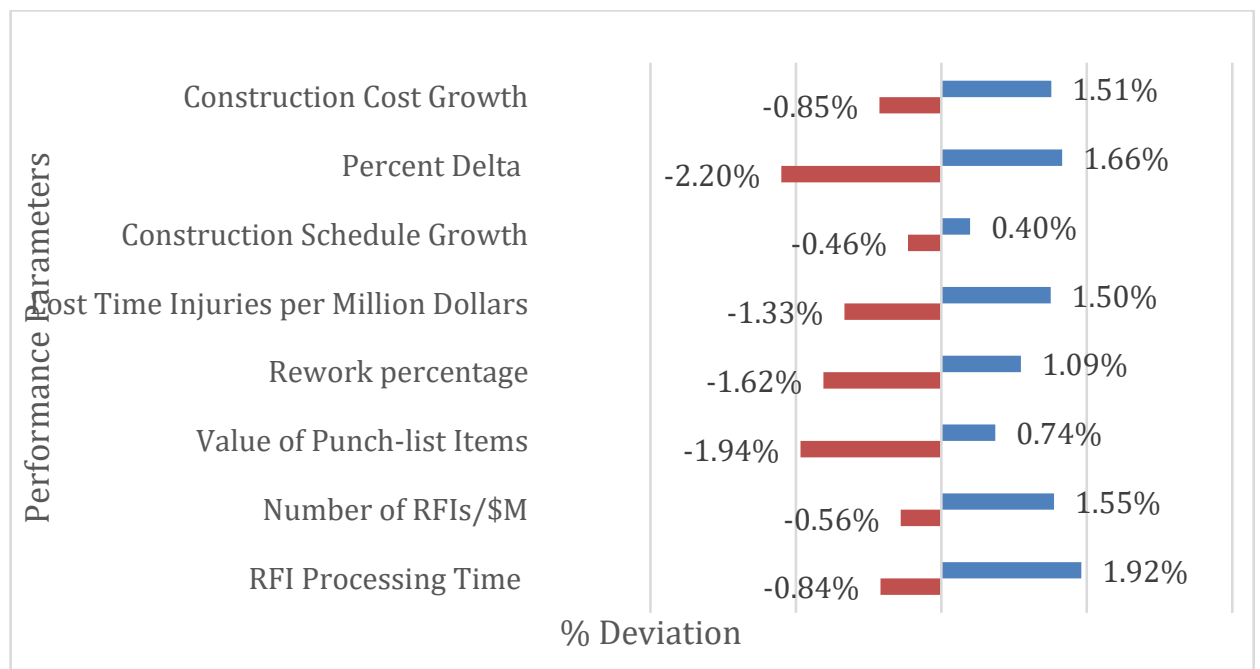

Figure 53: Maximum and minimum deviations of the PPA weights

The maximum, minimum, and total deviation in each of the weights of the performance metrics are computed, as shown in Table 34. The maximum and minimum total deviation of the eight-performance metrics weight were found to be $3.86 \%$ and $0.85 \%$ respectively.

Table 34: Maximum, Minimum and Total Deviations of the PPA Weights

\begin{tabular}{|l|l|l|c|}
\hline & $\begin{array}{l}\text { Maximum } \\
\text { Deviation }\end{array}$ & $\begin{array}{l}\text { Minimum } \\
\text { Deviation }\end{array}$ & $\begin{array}{c}\text { Total } \\
\text { Deviation }\end{array}$ \\
\hline
\end{tabular}




\begin{tabular}{|l|l|l|l|}
\hline Construction Cost Growth & $1.51 \%$ & $-0.85 \%$ & $2.36 \%$ \\
\hline Percent Delta & $1.66 \%$ & $-2.20 \%$ & $3.86 \%$ \\
\hline Construction Schedule Growth & $0.40 \%$ & $-0.46 \%$ & $0.85 \%$ \\
\hline $\begin{array}{l}\text { Lost Time Injuries per Million } \\
\text { Dollars }\end{array}$ & $1.50 \%$ & & \\
\hline Rework Percentage & $1.09 \%$ & $-1.33 \%$ & $2.84 \%$ \\
\hline Value of Punch-list Items & $0.74 \%$ & $-1.62 \%$ & $2.71 \%$ \\
\hline Number of RFIs/\$M & $1.55 \%$ & $-0.56 \%$ & $2.68 \%$ \\
\hline RFI Processing Time & $1.92 \%$ & $-0.84 \%$ & $2.11 \%$ \\
\hline
\end{tabular}

\subsection{Summary and Conclusions}

This chapter discussed the development of the mathematical formulation of the Project Performance Assessment (PPA) score for the different projects by combining eight key performance metrics spanning over six performance areas. The results showed that the top three metrics are: construction schedule growth, construction cost growth, and \% delta having 18.43\%, $17.38 \%$, and $15.22 \%$, respectively. On the other hand, the percentage of rework and the number of lost-time injuries per 1 million dollars have the lowest contribution to calculating the PPA score. K-means clustering was then performed to benchmark successful projects from less-thansuccessful projects and the cluster plot revealed the distinct classification of projects with limited overlap between the clusters. The study found that successful projects (cluster 1 ) have PPA scores with a range of 56\% - 95\%, while less-than-successful projects (cluster 2) have PPA scores with a range of 5\% - 55\%. Further analysis was conducted to verify that the selected metrics provide a substantial contribution to the PPA model by checking the correlation coefficient. Thus, none of the metrics were found to have a negligible correlation coefficient with PPA scores i.e. performance metrics have a $p$-value greater than 0.05 . Most of the performance metrics have moderate and strong correlation coefficients, but very few performance metrics have weak correlation coefficient, such as lost time injuries per millions of dollars and rework percentages, yet their correlation coefficients are significant since the $p$-value is less than 0.05 . Moreover, the 
Wilcoxon rank-sum test demonstrated that the data provides evidence to reject the null hypothesis at a $5 \%$ significance level, thus concluding at the $95 \%$ confidence level that there is statistically significant evidence that all the performance metrics studied vary across the status of a project. Finally, model robustness was performed for validating the PPA model to check that the results are not highly determined by changes in the dataset. It was found that the PPA model has a MAPE of $7.0 \%$ and the maximum and minimum total deviation of the eight-performance metrics weight were found to be $3.86 \%$ and $0.85 \%$ respectively. 


\section{Chapter 5 Quantitative Model of Project Competency}

Chapter 5 discusses the development of the mathematical formulation of the competency category-specific scores. The competency category-specific scores incorporate a set of factors with varying degrees of impact (i.e. weights) on project performance. The objectives of this chapter are to: 1) calculate the relative impact of the various competency factors to each category, spanning nine categories: alignment \& team integration, planning \& design, procurement management, risk management, health, safety \& environmental, quality management, labor productivity, execution \& commissioning, and change management \& project control; 2) compute a customized competency category-specific score from various competency factors; 3) identify objective databased weights for these factors; and therefore, alleviate the subjectivity associated with experts' judgment regarding the factors relative importance to one another; and 4) assess the overall effect of increased each competency category-specific score on the likelihood of the project being successful or less-than-successful.

\subsection{Statistical/Mathematical Methodology}

The section is divided into two parts. The first part discusses the pairwise correlation used to eliminate the problem of multicollinearity. The second part presents the top competency factors contributing to the PPA score using a statistical modeling technique called Spearman's Correlation test. In this way, only the most important competency factors are included in the model to effectively understand the dependent variable in the mathematical model and ensure its reliability.

Similar to what was done in chapter 4, before developing the mathematical formulation, multicollinearity need to be eliminated to avoid misleading results. First, collinear independent variables is identified and then remove by performing a pairwise correlation. Same cutoff 
coefficient was used as in chapter 4 and the pairwise correlation was conducted to remove competency factors with correlations coefficients $>0.75$ or $<-0.75$.

The second stage is to determine the top competency factors contributing to the PPA score using a statistical modeling technique called Spearman's Correlation test. Notably, the independent variables (competency factors) are nominal data and the dependent variable (the PPA score) is also found to be non-normal, based on normality checks, as previously illustrated in chapter 4 . Therefore, Spearman's Correlation test was used to statistically test the correlation between each of the competency factors and the PPA score. This is done by performing a hypothesis test for this correlation, such that the null hypothesis states that there is no correlation between the competency factors and the PPA score, while the alternative hypothesis states that correlation exists. Using Spearman's Correlation test for the hypothesis testing produces a p-value, which can be thought of as the probability of having no statistical correlation between the competency factors and the PPA score. Accordingly, a p-value of less than 0.05 demonstrates that the data provides evidence to reject the null hypothesis at a 5\% significance level, thus concluding at a 95\% confidence level that there is a statistically significant correlation between the competency factors and the PPA score. A second statistical metric is produced from this analysis is the Spearman's Rho correlation coefficient, which measures the relationship between each of the competency factors and the PPA score, so that it has a value of between -1 and 1 . A 0 means there is no relationship between the variables at all, while -1 or 1 means that there is a perfect negative or positive correlation.

The same mathematical model that was developed by Labib (2019), as described in chapter 4, was used for competency factors analysis. Importantly, the competencies are divided among categories, and each category has a different number of competencies. Thus, treating all competencies at once would result in overestimating/underestimating problems of the total weight 
of the competency categories that have a greater/smaller number of factors than others. Therefore, the developed mathematical model is used to calculate the relative impact of the various competency factors to each category and compute a customized competency category-specific score from various competency factors. The main aim is to maximize the sum of the square of the correlation between each of the individual competency factors and the competency categoryspecific scores.

\subsection{Competency Category-specific Scores Results}

\subsubsection{Alignment \& Team Integration}

The figure below shows the pairwise correlation matrix for alignment \& team integration factors. These results show AL\&T 11 and AL\&T 12 are highly correlated to each other with a coefficient of 0.752 , which illustrates that if the roles and responsibilities of the project team are well-defined, the more likely they will be effectively communicated to the project team. Then, a correlation test is conducted to analyze the highly correlated factors with the PPA score to determine which factor will be considered in the model; otherwise, these factors will not be considered. 


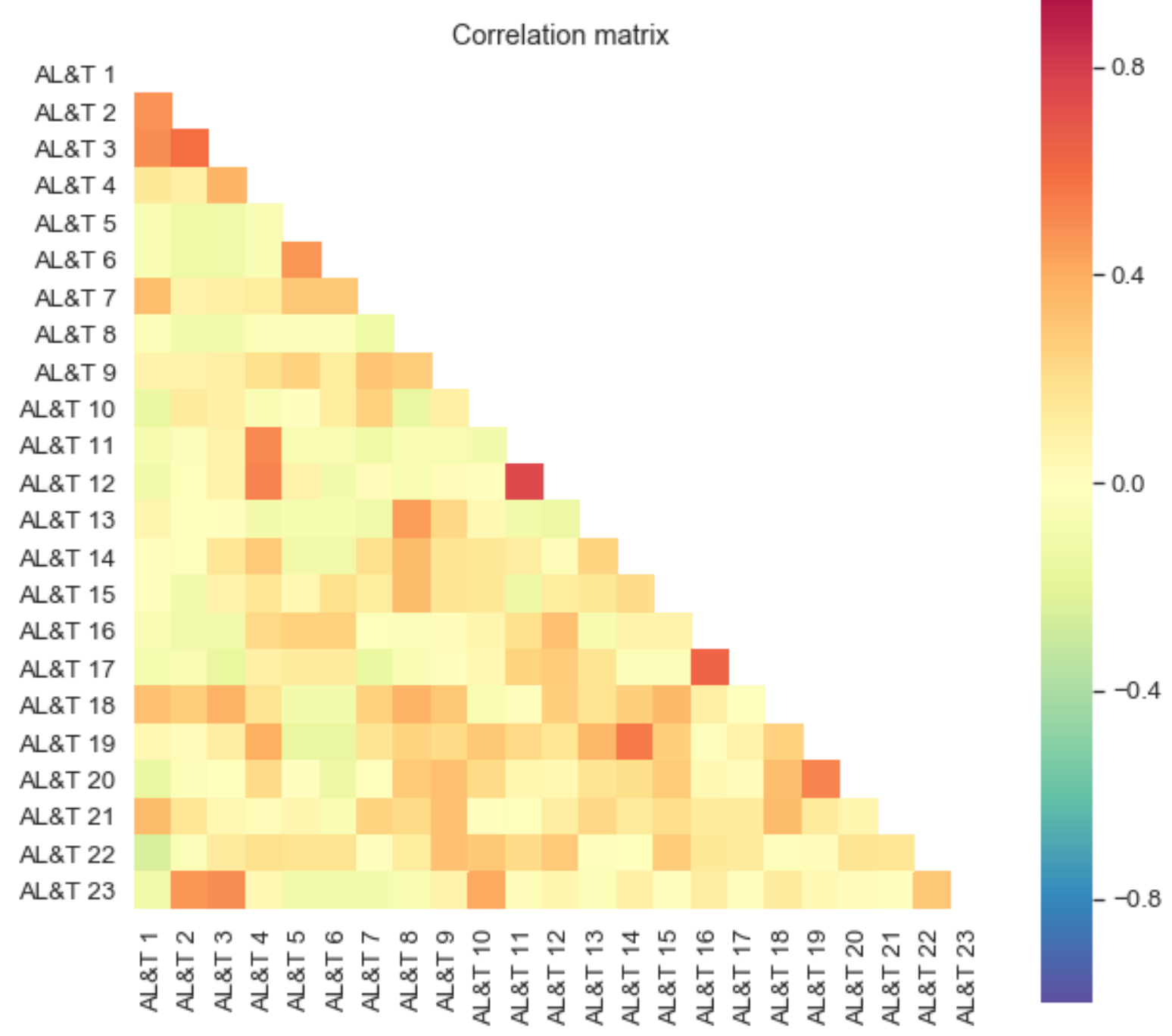

Figure 54: Correlation matrix for Alignment \& Team Integration factors

The table of descriptive statistics below shows the top competency factors contributing to the PPA score, the coefficient of correlation, and the $p$-value. 
Table 35: Correlation of Alignment \& Team Integration factors vs PPA score

\begin{tabular}{|l|l|c|c|}
\hline Code & Factor & Correlation to PPA & P-value \\
\hline AL\&T 1 & Project objectives defined by team members & 0.318 & 0.016 \\
\hline AL\&T 8 & $\begin{array}{l}\text { Construction personnel are included in project team from the } \\
\text { preconstruction phase. }\end{array}$ & 0.264 & 0.047 \\
\hline AL\&T 11 & Project team roles and responsibilities are well defined & 0.288 & 0.030 \\
\hline AL\&T 18 & Project team formed in a timely manner & 0.310 & 0.019 \\
\hline AL\&T 19 & Project team has all required skills & 0.318 & 0.016 \\
\hline AL\&T 20 & Project team has the expertise to take on the project & 0.441 & 0.001 \\
\hline
\end{tabular}

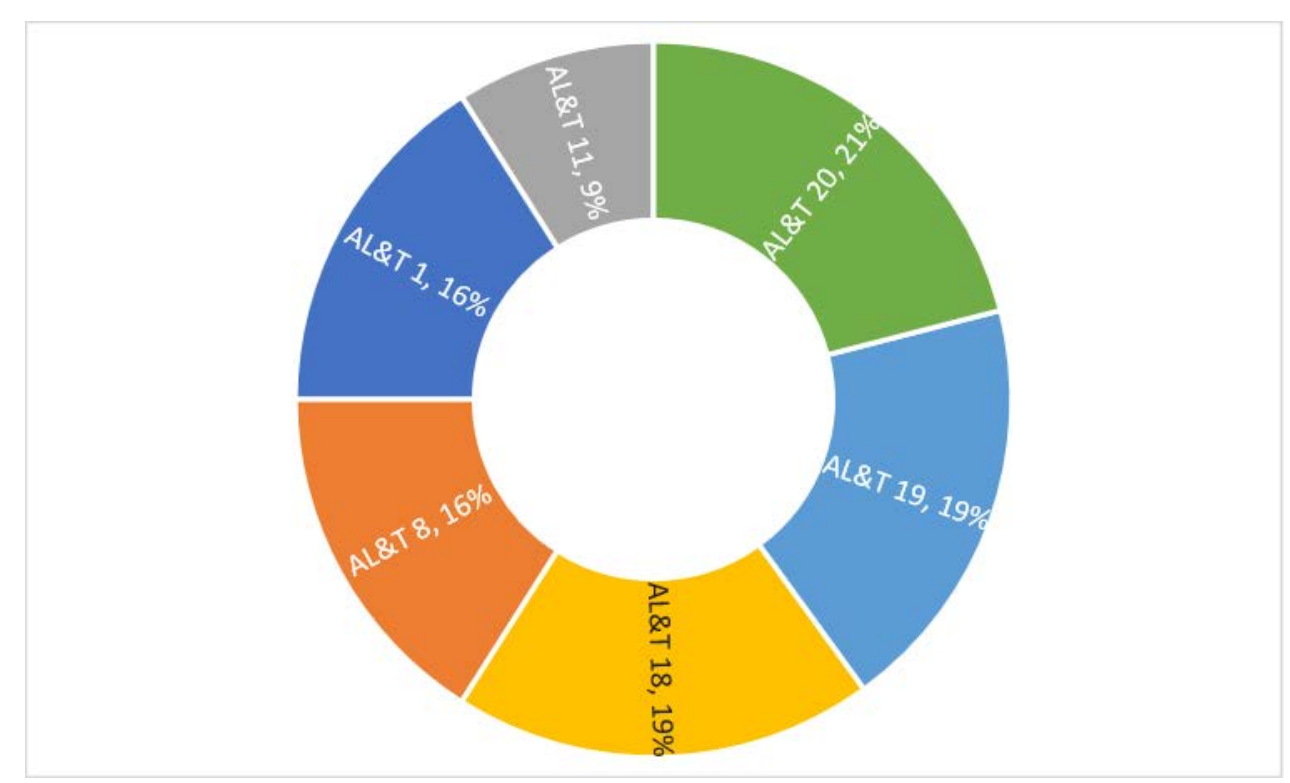

Figure 55: Weights of subcategories under Alignment \& Team Integration factors

A simplified equation was developed to accommodate the unstandardized data. The aggregation of this transformation for the Alignment \& Team integration factors resulted in the constant term. The results of the Alignment \& Team integration score are in a standardized form, i.e. a value that can be positive or negative. For better visualization and comparison of projects to one another, the authors scaled the generated Alignment \& Team integration score to have a range between 5\% to $95 \%$. A range of $[5,95 \%]$ was chosen instead of $[0,100 \%]$, as it is not reasonable to presume the worst possible project performance to be $0 \%$ and the best possible project performance to be $100 \%$. Therefore, the equation below eliminates errors that stem from 
standardization, while it also provides a user-friendly methodology to compute the final Alignment \& Team integration score of a range between $5 \%$ to $95 \%$ :

$A L \& T$ Score $=0.05+0.212$ AL\&T $1+0.119$ AL\&T $8+0.061$ AL\&T $11+0.200$ AL\&T $18+$ 0.140 AL\&T $19+0.168$ AL\&T 20

A Q-Q plot and Shapiro-Wilk test are conducted to assess the normality assumption. The following Q-Q plot shows a clear departure from normality at the overall level of the dataset.
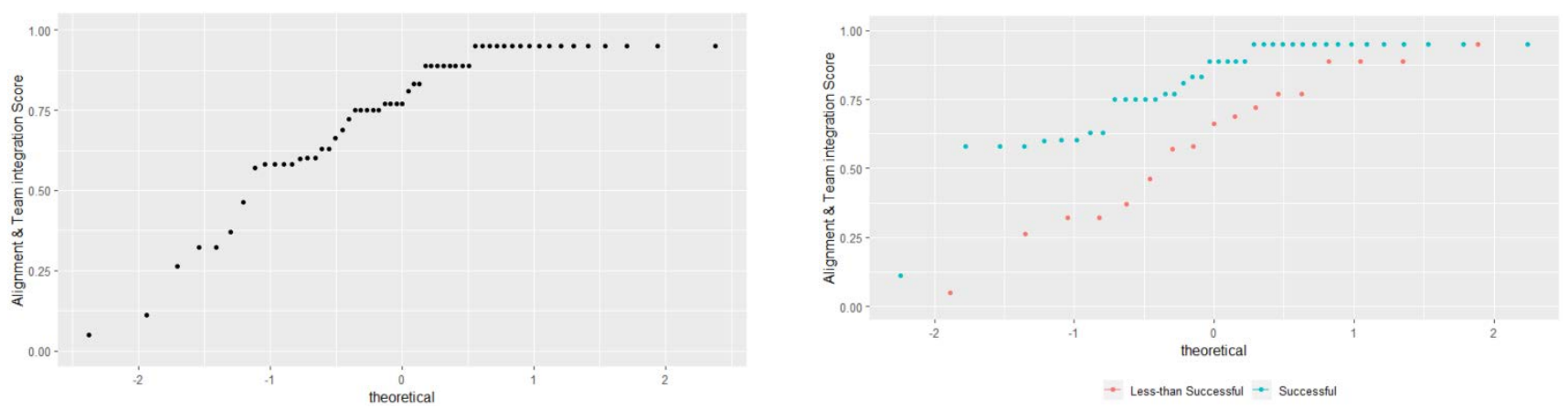

Figure 56: Q-Q Plot of Alignment \& Team Integration score

As with the other analysis, the normality assumption is not valid, as it was confirmed at a $95 \%$ confidence level by the Shapiro-Wilk test, which is shown in the table below.

Table 36: Shapiro-Wilk test for Alignment \& Team Integration score 


\begin{tabular}{ccc}
\hline \hline Overall & 0.000 & Significant \\
Successful Projects & 0.000 & Significant \\
Less-than-successful Projects & 0.349 & Not Significant \\
\hline \hline
\end{tabular}

The differences between successful and less-than-successful projects are shown by using box-and-whisker plots. It is visually evident that less-than-successful projects have higher variability than successful ones, as it was illustrated from the level of compaction of the boxplot. That was also evident from the Fligner-Killeen test returning a p-value of 0.033 , which rejects the null hypothesis of equal variance among project status, confirming the previous suggestion of higher variability among less-than-successful projects at a 95\% confidence level. The Wilcoxon rank-sum test returned a p-value of 0.002 , which demonstrates that the data provides evidence to reject the null hypothesis at a 5\% significance level, thus concluding at a 95\% confidence level that there is statistically significant evidence that Alignment \& Team integration score varies across the status of the project. Thus, it shows that successful projects usually focus on scope alignment and team formation, contrary to less-than-successful projects, which might not give scope alignment and team formation enough attention. 


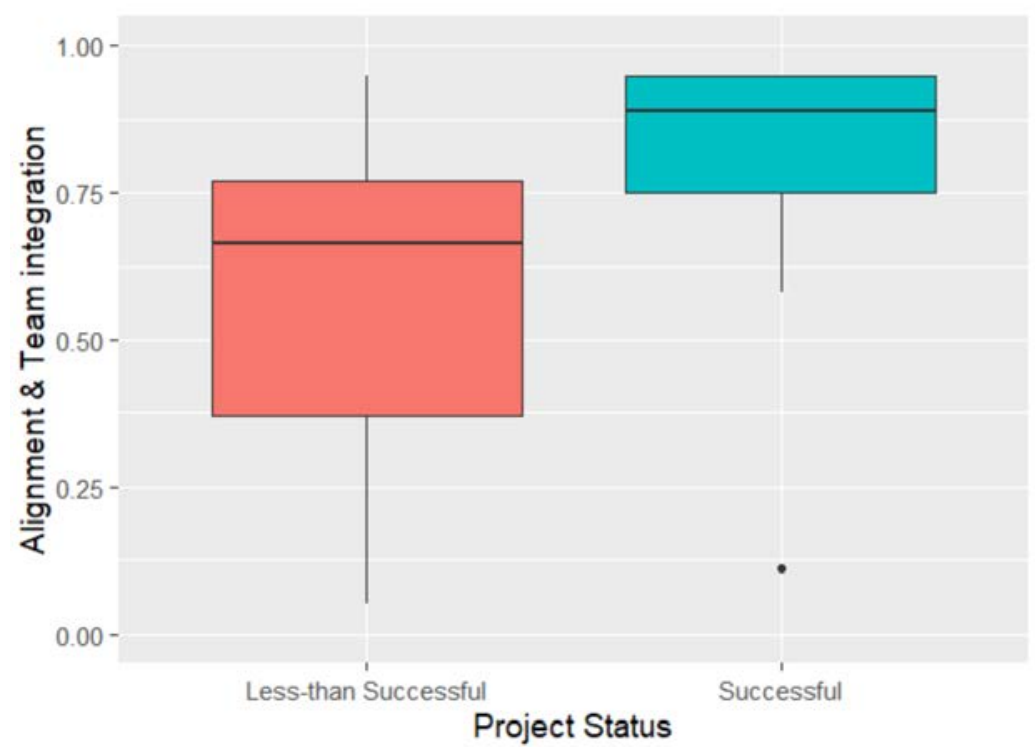

Figure 57: Boxplot of Alignment \& Team Integration score

The table of descriptive statistics below shows that the median/mean for Alignment \& Team integration score for successful projects (88.9/80.8\%) is higher than less-than-successful projects (66.3/59.8\%), which shows the importance of highly considering the scope alignment and team formation for project success.

Table 37: Descriptive statistics for Alignment \& Team Integration score

\begin{tabular}{ccc}
\hline \hline Statistical Test & Successful Projects & Less-than-successful Projects \\
\hline \hline Mean & $80.8 \%$ & $59.8 \%$ \\
Maximum & $95.0 \%$ & $95.0 \%$ \\
$75 \%$ Quantile & $95.0 \%$ & $77.0 \%$ \\
50\% Quantile & $88.9 \%$ & $66.3 \%$ \\
25\% Quantile & $74.9 \%$ & $36.9 \%$ \\
Minimum & $11.1 \%$ & $5.0 \%$ \\
\hline \hline
\end{tabular}




\subsubsection{Planning \& Design}

The figure below shows the pairwise correlation matrix for Planning \& Design factors. These results show that there is no occurrence of high intercorrelations among independent variables in accordance with the cutoff value.

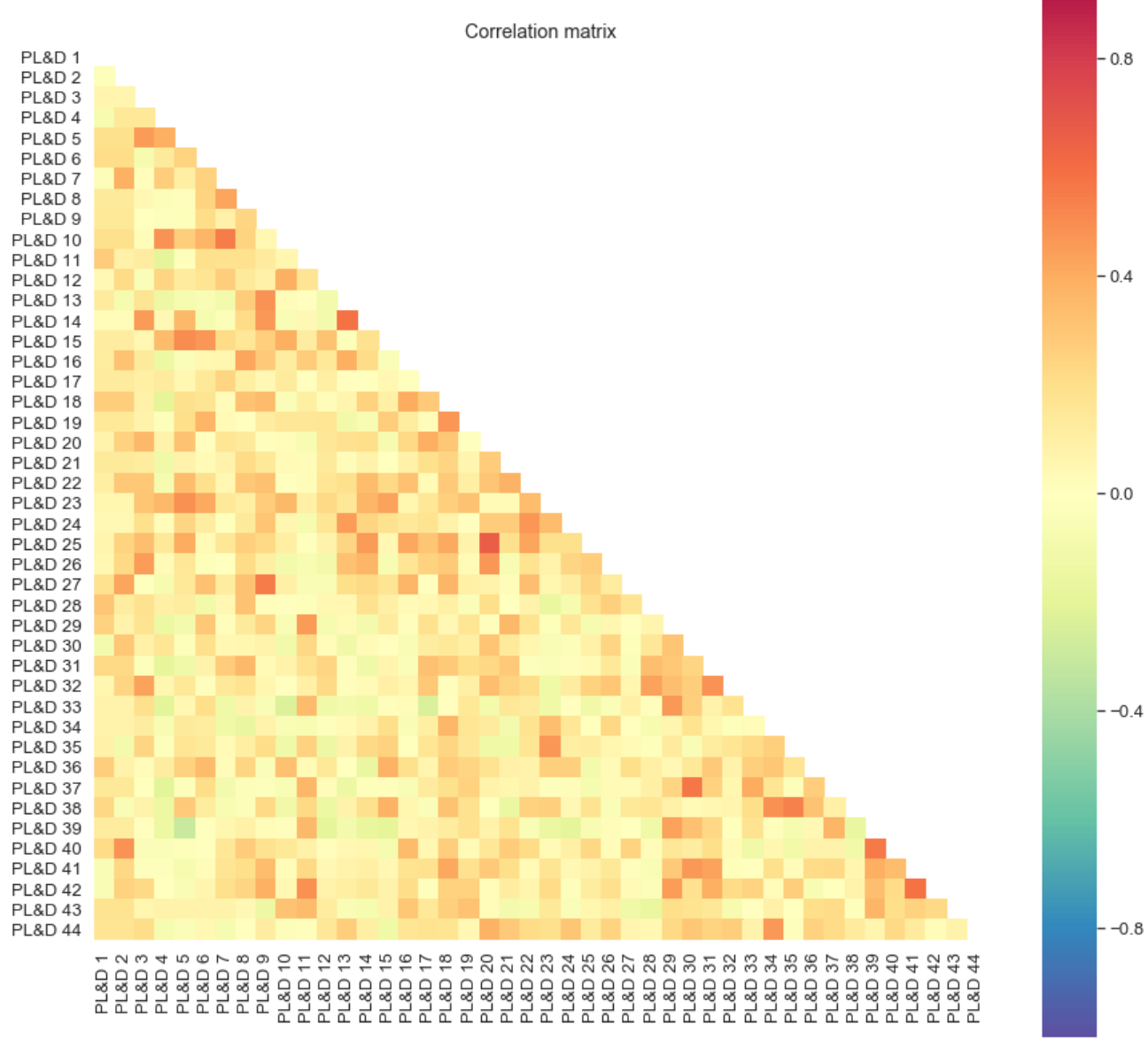

Figure 58: Correlation matrix for Planning \& Design factors 
The table of descriptive statistics below shows the top competency factors contributing to the

PPA score, the coefficient of the correlation, and the $p$-value.

Table 38: Correlation of Planning \& Design factors vs PPA score

\begin{tabular}{|c|c|c|c|}
\hline Code & Factor & $\begin{array}{c}\text { Correlation to } \\
\text { PPA }\end{array}$ & P-value \\
\hline PL\&D 3 & Master schedule considers subcontractor schedules & 0.341 & 0.010 \\
\hline PL\&D 17 & Labor resource requirements are supported in the project & 0.342 & 0.009 \\
\hline PL\&D 18 & Hold points/handoffs are identified & 0.327 & 0.013 \\
\hline PL\&D 20 & Overmanning considered in the schedule & 0.335 & 0.011 \\
\hline PL\&D 25 & Resource leveling (especially manpower) is considered in schedule & 0.325 & 0.014 \\
\hline PL\&D 30 & Standards and specifications are well defined & 0.300 & 0.023 \\
\hline PL\&D 31 & IFC drawings are issued to support the construction activity & 0.368 & 0.005 \\
\hline PL\&D 32 & Schedule for design deliverables is compatible with construction sequence & 0.393 & 0.002 \\
\hline PL\&D 33 & Clash detection checks are implemented & 0.280 & 0.035 \\
\hline PL\&D 35 & Design incorporates commissioning and startup & 0.418 & 0.001 \\
\hline PL\&D 36 & The different disciplines of design are well coordinated & 0.278 & 0.036 \\
\hline PL\&D 37 & Engineering responsibilities are well defined & 0.310 & 0.019 \\
\hline PL\&D 38 & Design incorporated operability & 0.386 & 0.003 \\
\hline
\end{tabular}

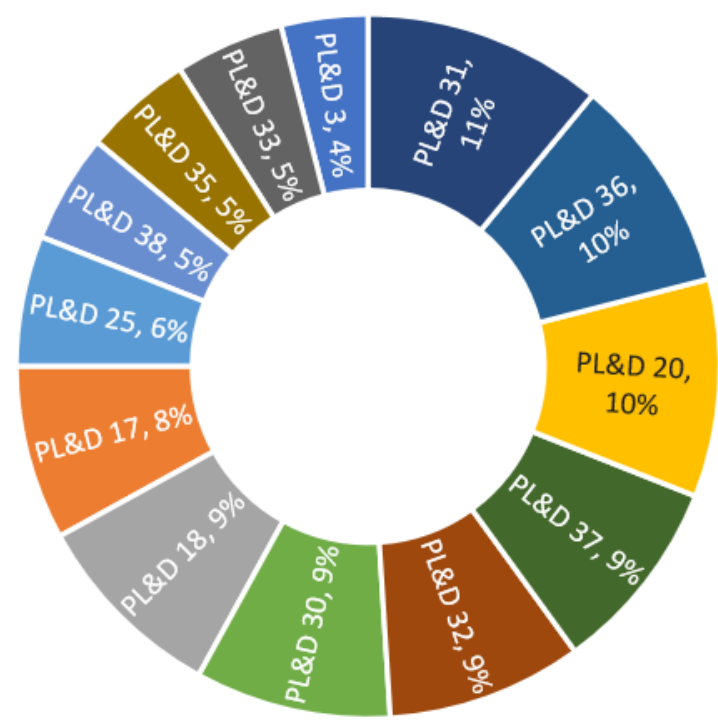

Figure 59: Weights of subcategories under Planning \& Design factors 
A simplified equation was developed to accommodate the unstandardized data. The aggregation of this transformation for the Planning \& Design factors resulted in the constant term. The results of the Planning \& Design score are in a standardized form, i.e. a value that can be positive or negative. For better visualization and comparison of projects to one another, the authors scaled the generated Planning \& Design score to have a range between 5\% to 95\%. A range of [5, 95\%] was chosen instead of $[0,100 \%]$, as it is not reasonable to presume the worst possible project performance to be $0 \%$ and the best possible project performance to be $100 \%$. Therefore, the equation below eliminates errors that stem from standardization, while it also provides a userfriendly methodology to compute the Planning \& Design score of a range between 5\% to 95\%:

PL\&D Score $=-0.150+0.042$ PL\&D $3+0.096$ PL\&D $17+0.092$ PL\&D $18+$

0.111 PL\&D $20+0.067$ PL\&D $25+0.103$ PL\&D $30+0.109$ PL\&D $31+0.095$ PL\&D $32+$ $0.054 P L \& D 33+0.044 P L \& D 35+0.106$ PL\&D $36+0.104 P L \& D 37+0.076$ PL\&D 38

A Q-Q plot and Shapiro-Wilk test are conducted to assess the normality assumption. The following Q-Q plot shows a clear departure from normality at the overall level of the dataset.
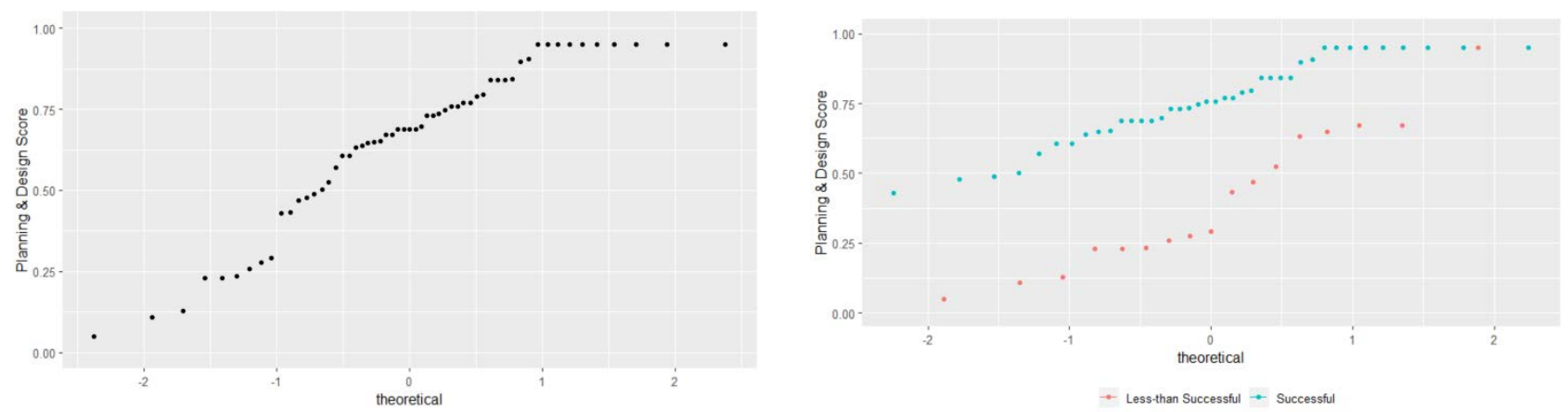

Figure 60: Q-Q Plot of Planning \& Design score 
As with the other analysis, the normality assumption is not valid, as it was confirmed at a 95\% confidence level by the Shapiro-Wilk test, which is shown in the table below.

Table 39: Shapiro-Wilk test for Planning \& Design score

Parameter

p-value

0.001

0.022

Successful Projects

Less-than-Successful Projects
Significance at 95\% Confidence Level

\begin{tabular}{ccc}
\hline \hline Overall & 0.001 & Significant \\
Successful Projects & 0.022 & Significant \\
Less-than-Successful Projects & 0.260 & Not Significant \\
\hline \hline
\end{tabular}

The differences between successful and less-than-successful projects are shown by using box-and-whisker plots. It is visually evident that less-than-successful projects have higher variability than successful ones, as it was illustrated from the level of compaction of the boxplot. That was also evident from the Fligner-Killeen test returning a p-value of 0.04 , which rejects the null hypothesis of equal variance among project status, confirming the previous suggestion of higher variability among less-than-successful projects at a 95\% confidence level. The Wilcoxon rank-sum test returned a p-value of 0.000 , which demonstrates that the data provides evidence to reject the null hypothesis at a 5\% significance level, thus concluding at a 95\% confidence level that there is statistically significant evidence that Planning \& Design score varies across the status of the project. Thus, it shows that successful projects usually focus on the preconstruction phase (planning and design aspects), contrary to less-than-successful projects, which might not plan for the preconstruction phase effectively. 


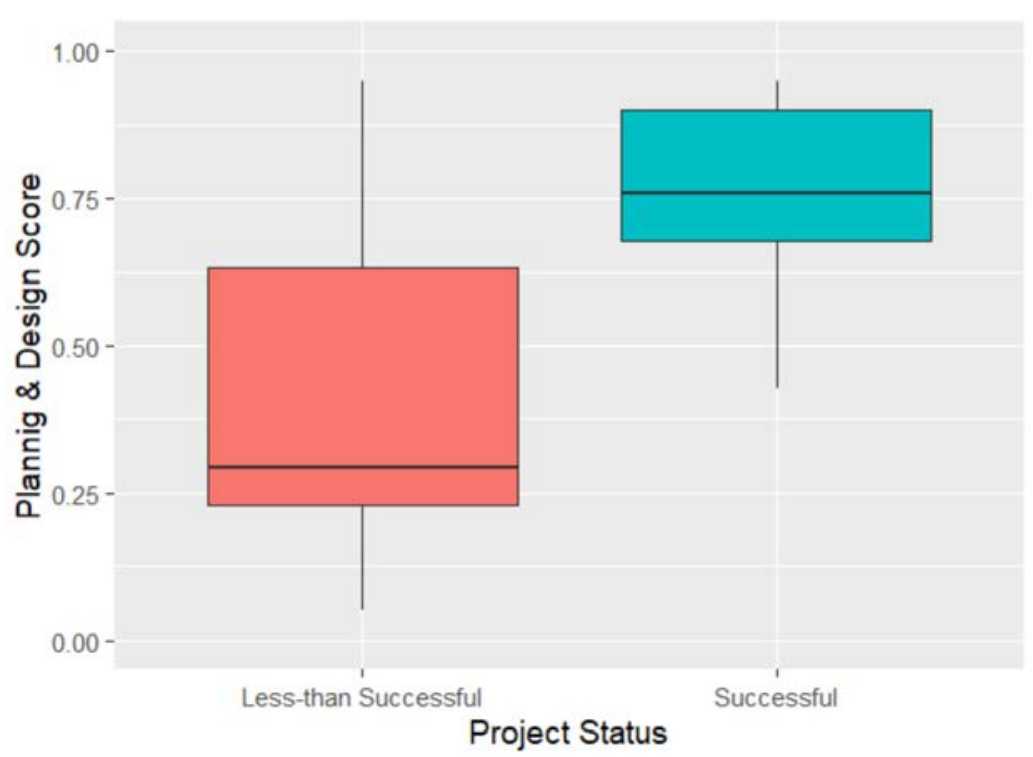

Figure 61: Boxplot of Planning \& Design score

The table of descriptive statistics below shows that the median/mean for Planning \& Design score for successful projects $\mathbf{( 7 5 . 8 / 7 5 . 9 \% )}$ is higher than less-than-successful projects (29.3/40.0\%), which shows the importance of highly considering the preconstruction phase (planning and design aspects) for project success.

Table 40: Descriptive statistics for Planning \& Design score

\begin{tabular}{ccc}
\hline \hline Statistical Test & Successful Projects & Less-than-successful Projects \\
\hline \hline Mean & $75.9 \%$ & $40.0 \%$ \\
Maximum & $95.0 \%$ & $95.0 \%$ \\
$75 \%$ Quantile & $89.9 \%$ & $63.2 \%$ \\
$50 \%$ Quantile & $75.8 \%$ & $29.3 \%$ \\
$25 \%$ Quantile & $68.0 \%$ & $22.9 \%$ \\
Minimum & $42.8 \%$ & $5.0 \%$ \\
\hline \hline
\end{tabular}




\subsubsection{Procurement Management}

The figure below shows the pairwise correlation matrix for Procurement Management factors. These results show there is a high occurrence of intercorrelations among Proc. 5,6,8, and 9 with a coefficient exceeding 0.75 , which illustrates that having a good management plan for tools and equipment in place is necessary and depends on having good placement, storage, control, and maintenance plans. Then, a correlation test is conducted to analyze the highly correlated factors with the PPA score to determine which factor will be considered in the mode; otherwise, these factors will not be considered.

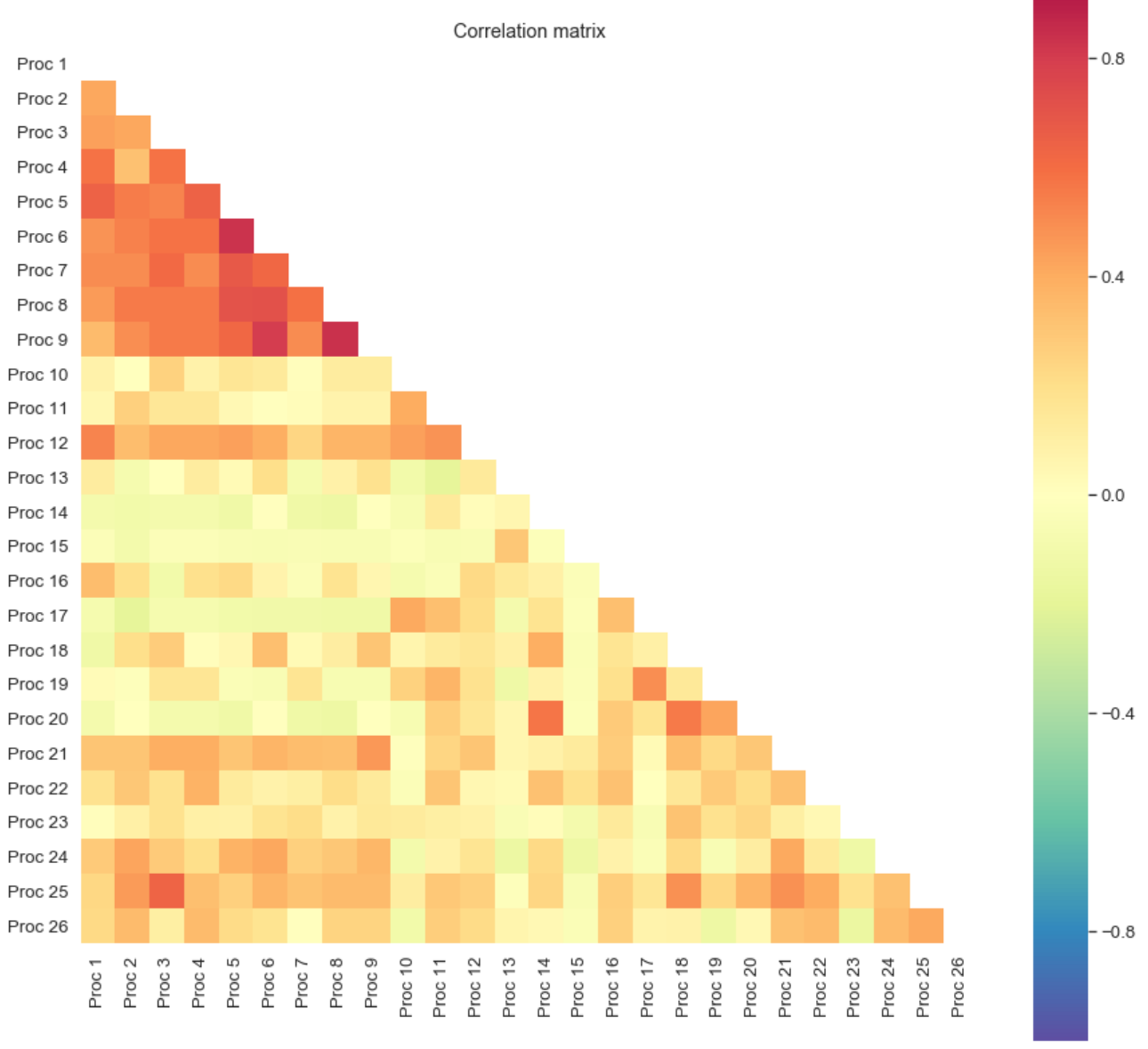

Figure 62: Correlation matrix for Procurement Management factors 
The table of descriptive statistics below shows the top competency factors contributing to the

PPA score, the coefficient of correlation, and the $p$-value.

Table 41: Correlation of Procurement Management factors vs PPA score

\begin{tabular}{|l|l|c|c|}
\hline Code & Factor & Correlation to PPA & P-value \\
\hline Proc 12 & $\begin{array}{l}\text { Tools \& consumables are identified and secured before } \\
\text { construction }\end{array}$ & 0.281 & 0.034 \\
\hline Proc 18 & Storage plan for materials is secured & 0.446 & 0.001 \\
\hline Proc 23 & $\begin{array}{l}\text { All bulky materials needed for start of construction are } \\
\text { purchased \& on site }\end{array}$ & 0.379 & 0.004 \\
\hline
\end{tabular}

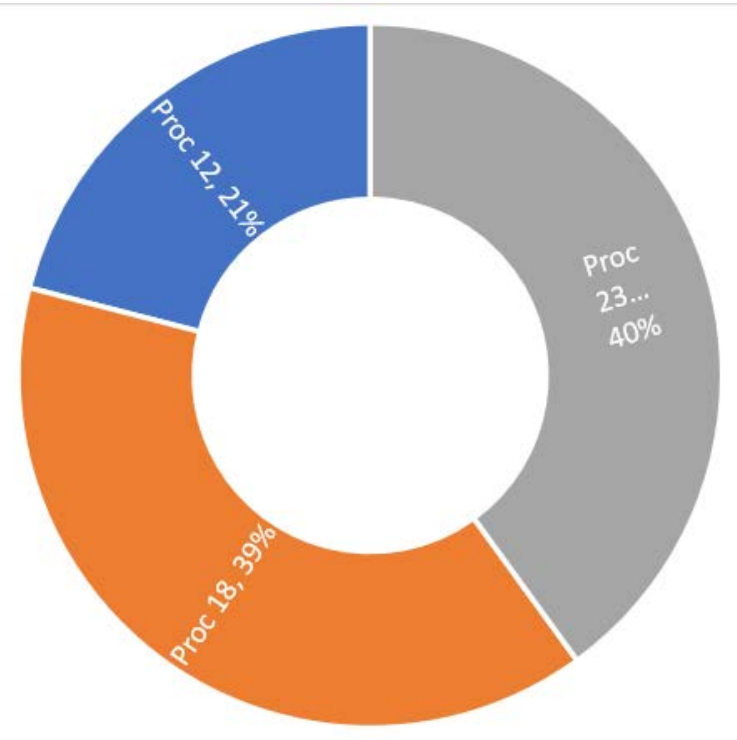

Figure 63: Weights of Subcategories under Procurement Management factors

A simplified equation was developed to accommodate the unstandardized data. The aggregation of this transformation for the Procurement Management factors resulted in the constant term. The results of the Procurement Management score are in a standardized form, i.e. a value that can be positive or negative. For better visualization and comparison of projects to one another, the authors scaled the generated Procurement Management score to have a range between $5 \%$ to $95 \%$. A range of $[5,95 \%]$ was chosen instead of $[0,100 \%]$, as it is not reasonable to presume the worst possible project performance to be $0 \%$ and the best possible project performance to be 
100\%. Therefore, the equation below eliminates errors that stem from standardization, while it also provides a user-friendly methodology to compute the Procurement Management score of a range between $5 \%$ to $95 \%$ :

Proc Score $=0.050+0.193$ Proc $12+0.419$ Proc $18+0.288$ Proc 23

A Q-Q plot and Shapiro-Wilk test are conducted to assess the normality assumption. The following Q-Q plot shows a clear departure from normality at the overall level of the dataset.
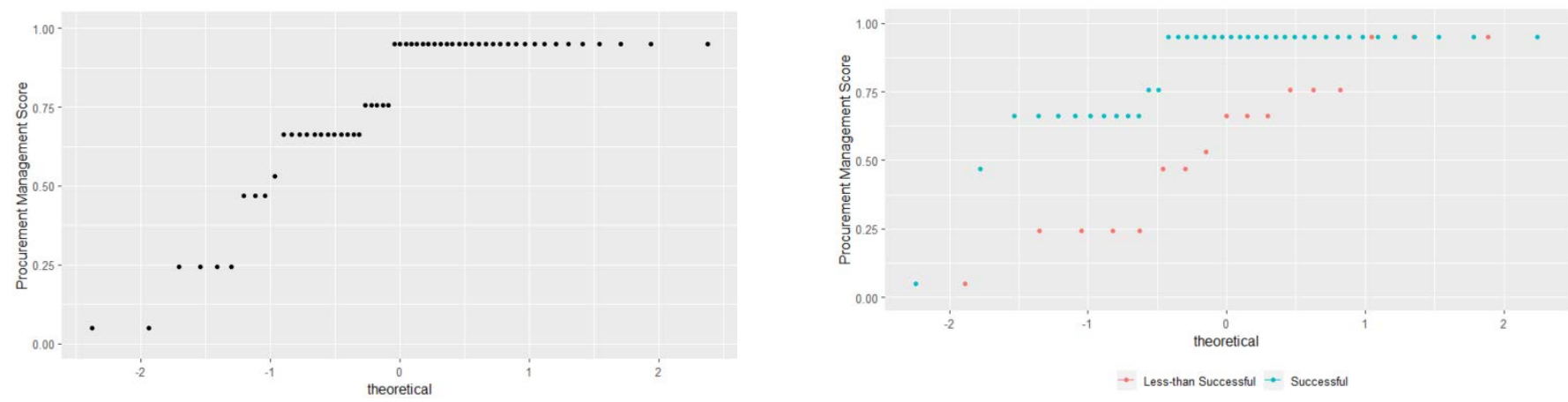

Figure 64: Q-Q plot of Procurement Management score

As with the other analysis, the normality assumption is not valid, as it was confirmed at a 95\% confidence level by the Shapiro-Wilk test, which is shown in the table below.

Table 42: Shapiro-Wilk test for Procurement Management score

\begin{tabular}{ccc}
\hline \hline Parameter & $p$-value & Significance at 95\% Confidence Level \\
\hline \hline Overall & 0.000 & Significant \\
Successful Projects & 0.000 & Significant \\
Less-than-successful Projects & 0.176 & Not Significant \\
\hline \hline
\end{tabular}


The differences between successful and less-than-successful projects are shown by using box-and-whisker plots. It is visually evident that less-than-successful projects have higher variability than successful ones, as it was illustrated from the level of compaction of the boxplot. That was also evident from the Fligner-Killeen test returning a p-value of 0.007 , which rejects the null hypothesis of equal variance among project status, confirming the previous suggestion of higher variability among less-than-successful projects at a 95\% confidence level. The Wilcoxon rank-sum test returned a p-value of 0.000 , which demonstrates that the data provides evidence to reject the null hypothesis at a 5\% significance level, thus concluding at a 95\% confidence level that there is statistically significant evidence that Procurement Management score varies across the status of the project. Thus, it shows that successful projects usually focus on preparing a purchase list for tools, materials, and equipment before the construction phase, contrary to lessthan-successful projects, which might not give a procurement plan for the whole project effectively.

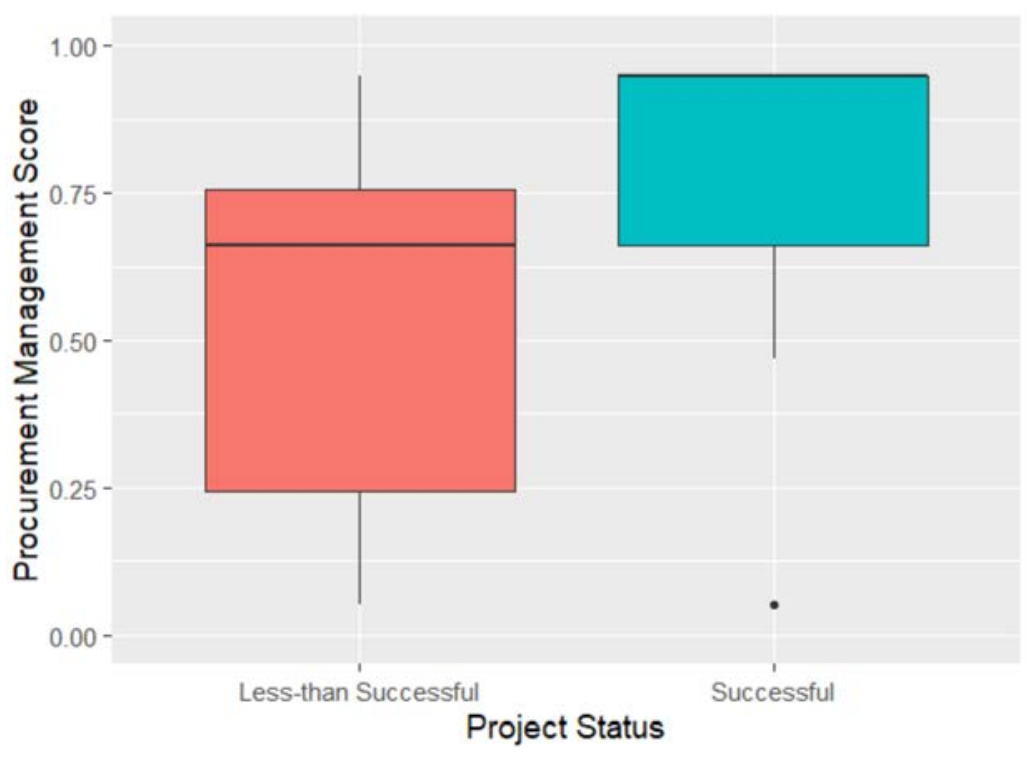

Figure 65: Boxplot of Procurement Management score 
The table of descriptive statistics below shows that the median/mean for Procurement Management score for successful projects (95.0/84.1\%) is higher than less-than-successful projects (66.2/56.4\%), which shows the importance of highly considering the procurement of tools, materials, and equipment before the construction phase for project success.

Table 43: Descriptive statistics for Procurement Management score

\begin{tabular}{ccc}
\hline \hline Statistical Test & Successful Projects & Less-than-successful Projects \\
\hline \hline Mean & $84.1 \%$ & $56.4 \%$ \\
Maximum & $95.0 \%$ & $95.0 \%$ \\
$75 \%$ Quantile & $95.0 \%$ & $75.7 \%$ \\
50\% Quantile & $95.0 \%$ & $66.2 \%$ \\
25\% Quantile & $66.2 \%$ & $24.3 \%$ \\
Minimum & $6.0 \%$ & $5.0 \%$ \\
\hline \hline
\end{tabular}




\subsubsection{Contract \& Risk Management}

The figure below shows the pairwise correlation matrix for Contract \& Risk Management factors. These results show a defining framework for risk monitoring and response in terms of cost and schedule are highly correlated to each other with a coefficient of 0.894 . Then, a correlation test is conducted to analyze the highly correlated factors with the PPA score to determine which factor will be considered in the model; otherwise, these factors will not be considered.

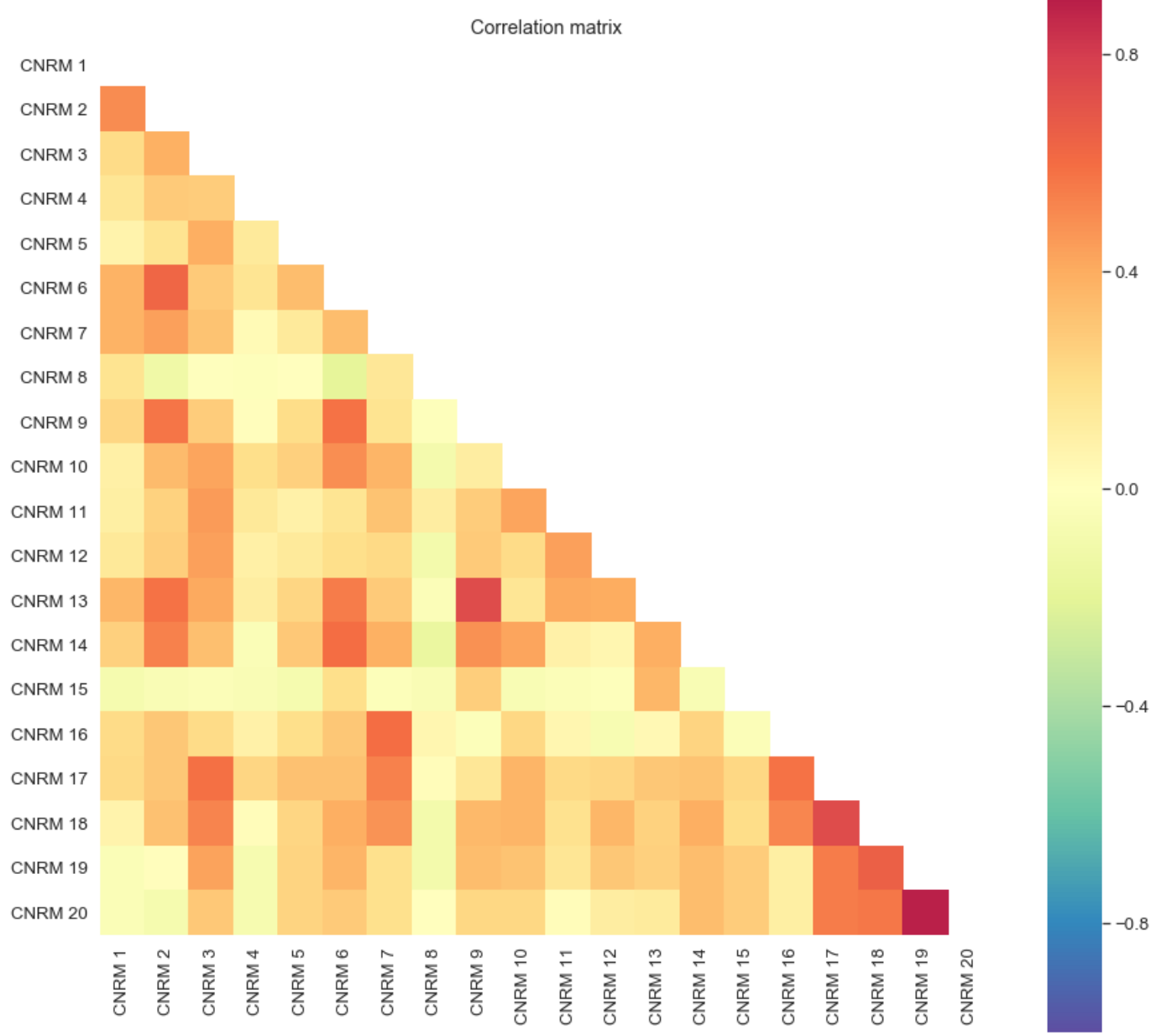

Figure 12: Correlation matrix for Contract \& Risk Management factors 
The table of descriptive statistics below shows the top competency factors contributing to the PPA score, the coefficient of the correlation, and the $p$-value.

Table 44: Correlation of Contract \& Risk Management Factors vs PPA score

\begin{tabular}{|l|l|c|c|}
\hline Code & Factor & $\begin{array}{c}\text { Correlation to } \\
\text { PPA }\end{array}$ & P-value \\
\hline CNRM 16 & $\begin{array}{l}\text { Risks impacting the construction phase of the project are } \\
\text { well-assessed }\end{array}$ & 0.314 & 0.018 \\
\hline CNRM 17 & Risk identification and evaluation process are well defined & 0.311 & 0.018 \\
\hline CNRM 18 & Risk analysis and investigation process are well defined & 0.330 & 0.012 \\
\hline CNRM 19 & $\begin{array}{l}\text { Risk monitoring and response framework are well defined in } \\
\text { terms of cost }\end{array}$ & 0.334 & 0.011 \\
\hline
\end{tabular}

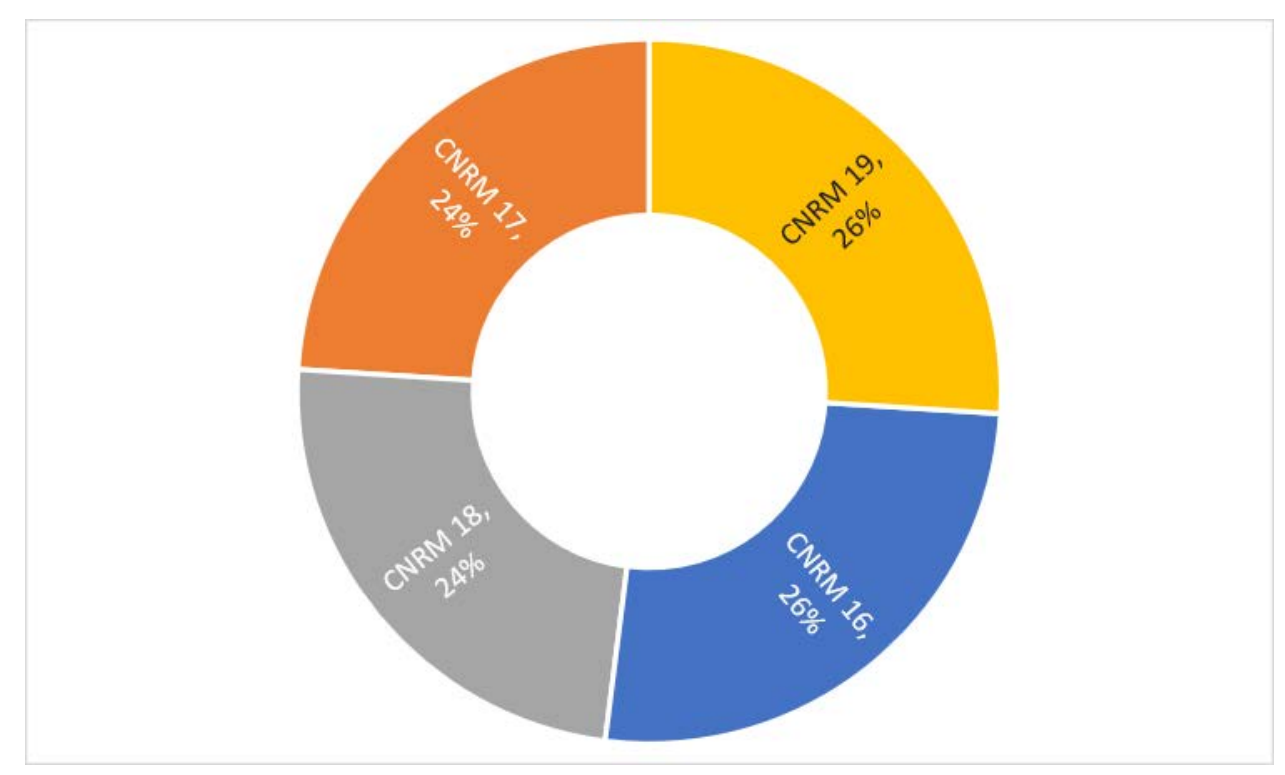

Figure 13: Weights of subcategories under Contract \& Risk Management factors

A simplified equation was developed to accommodate the unstandardized data. The aggregation of this transformation for the Contract \& Risk management factors resulted in the constant term. The results of the Contract \& Risk management score are in a standardized form, i.e. a value that can be positive or negative. For better visualization and comparison of projects to one another, the authors scaled the generated Contract \& Risk management score to have a range between $5 \%$ to $95 \%$. A range of $[5,95 \%]$ was chosen instead of $[0,100 \%]$, as it is not reasonable 
to presume the worst possible project performance to be $0 \%$ and the best possible project performance to be $100 \%$. Therefore, the equation below eliminates errors that stem from standardization, while it also provides a user-friendly methodology to compute the Contract \& Risk Management score of a range between 5\% to 95\%:

$$
\begin{gathered}
\text { CNRM Score }=0.050+0.242 \text { CNRM } 16+0.206 \text { CNRM } 17+0.202 \text { CNRM } 18+ \\
0.250 \text { CNRM } 19
\end{gathered}
$$

A Q-Q plot and Shapiro-Wilk test are conducted to assess the normality assumption. The following Q-Q plot shows a clear departure from normality at the overall level of the dataset.
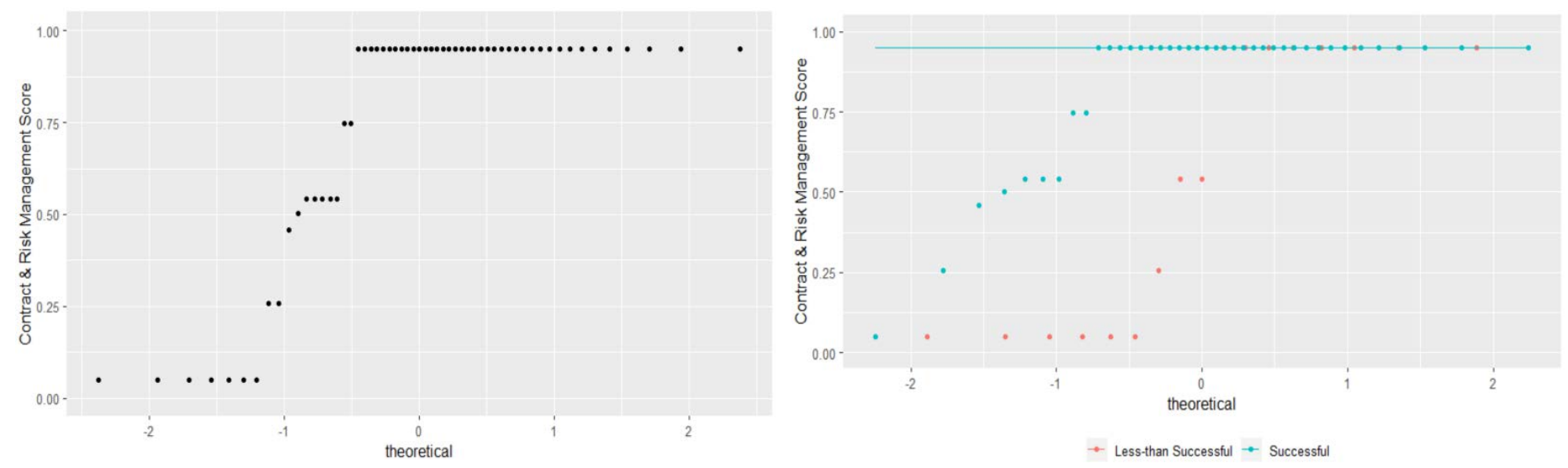

Figure 66: Q-Q plot of Contract \& Risk Management score 
As with the other analysis, the normality assumption is not valid, as it was confirmed at a 95\% confidence level by the Shapiro-Wilk test, which is shown in the table below.

Table 45: Shapiro-Wilk test for Contract \& Risk Management score
Parameter
p-value
Significance at 95\% Confidence Level

\begin{tabular}{ccc}
\hline \hline Overall & 0.000 & Significant \\
Successful Projects & 0.000 & Significant \\
Less-than-Successful Projects & 0.000 & Significant \\
\hline \hline
\end{tabular}

The differences between successful and less-than-successful projects are shown by using box-and-whisker plots. It is visually evident that less-than-successful projects have higher variability than successful ones, as it was illustrated from the level of compaction of the boxplot. That was also evident from the Fligner-Killeen test returning a p-value of 0.000, which rejects the null hypothesis of equal variance among project status, confirming the previous suggestion of higher variability among less-than-successful projects at a 95\% confidence level. The Wilcoxon rank-sum test returned a p-value of 0.006, which demonstrates that the data provides evidence to reject the null hypothesis at a 5\% significance level, thus concluding at a 95\% confidence level that there is statistically significant evidence that Contract \& Risk Management score varies across the status of the project. Thus, it shows that successful projects usually focus on having a good contract and risk management, contrary to less-than-successful projects, which might not give it enough attention. 


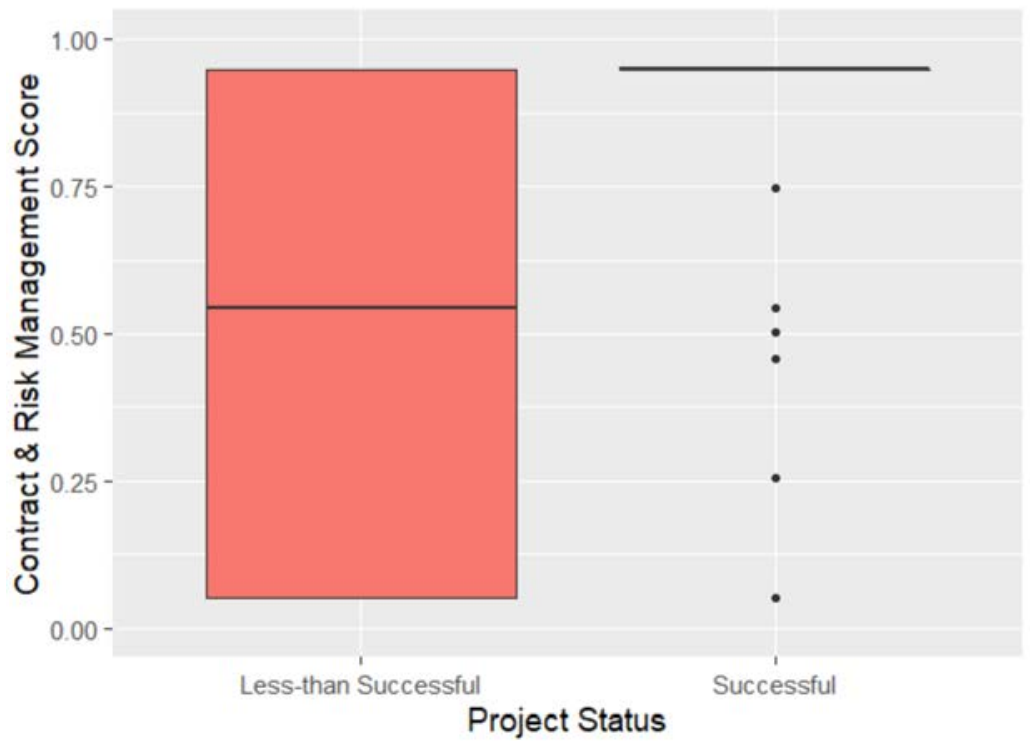

Figure 67: Boxplot of Contract \& Risk Management score

The table of descriptive statistics below shows that the median/mean for Contract \& Risk Management score for successful projects (95.0/84.6\%) is higher than less-than-successful projects (54.2/54.4\%), which shows the importance of having an effective contract and risk management for project success.

Table 46: Descriptive statistics for Contract \& Risk Management score

\begin{tabular}{ccc}
\hline \hline Statistical Test & Successful Projects & Less-than-successful Projects \\
\hline \hline Mean & $84.6 \%$ & $54.4 \%$ \\
Maximum & $95.0 \%$ & $95.0 \%$ \\
$75 \%$ Quantile & $95.0 \%$ & $95.0 \%$ \\
$50 \%$ Quantile & $95.0 \%$ & $54.2 \%$ \\
25\% Quantile & $95.0 \%$ & $5.0 \%$ \\
Minimum & $5.0 \%$ & $5.0 \%$ \\
\hline \hline
\end{tabular}




\subsubsection{Health, Safety, \& Environmental}

The figure below shows the pairwise correlation matrix for Health, Safety, \& Environmental (HSE) factors. These results show that there is no occurrence of high intercorrelations among independent variables in accordance with the cutoff value. The results show that none of the HSE factors will be considered to be parameters in the model, since the pvalue for the correlation test has a value greater than 0.05 , providing evidence to accept the null hypothesis at a 95\% confidence level that there is no statistically significant correlation between the HSE factors and the PPA score. This idea can be justified in that all the projects in the tested sample consider health and safety as a priority and thus it is not an aspect that differentiates between extraordinary projects from average ones.

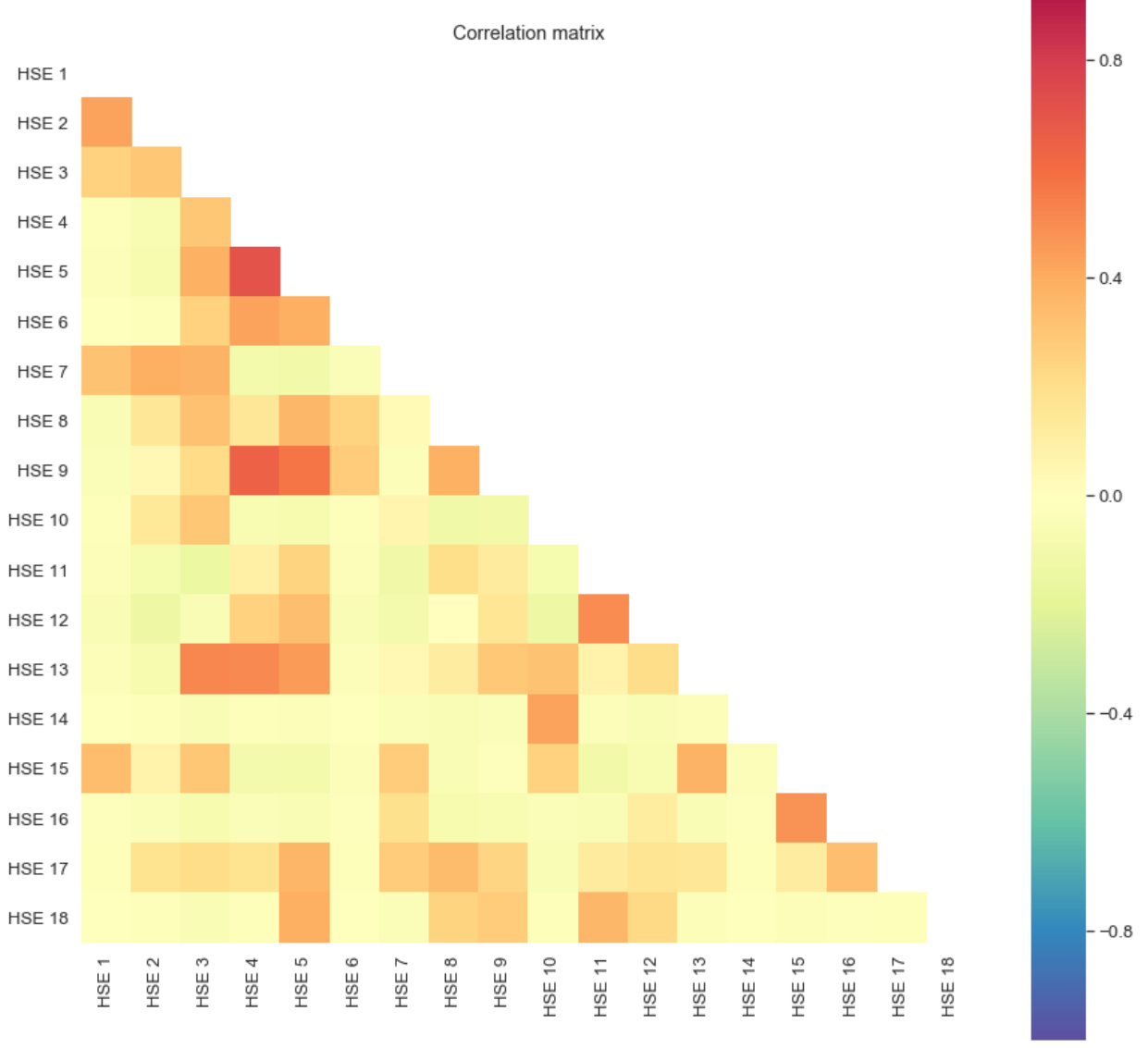

Figure 68: Correlation matrix for Health, Safety, \& Environmental factors 


\subsubsection{Quality Management}

The figure below shows the pairwise correlation matrix for Quality Management factors. These results show there is a high occurrence of intercorrelations among QM 1,2,7, and 8 with a coefficient exceeding 0.75, which illustrates that having a well-defined and effectively communicated quality assurance plan is very important, which is also dependent on defining and coordinating the roles and responsibilities of the quality inspection to the project team. Then, a correlation test is conducted to analyze the highly correlated factors with the PPA score to determine which factor will be considered in the mode; otherwise, these factors will not be considered.

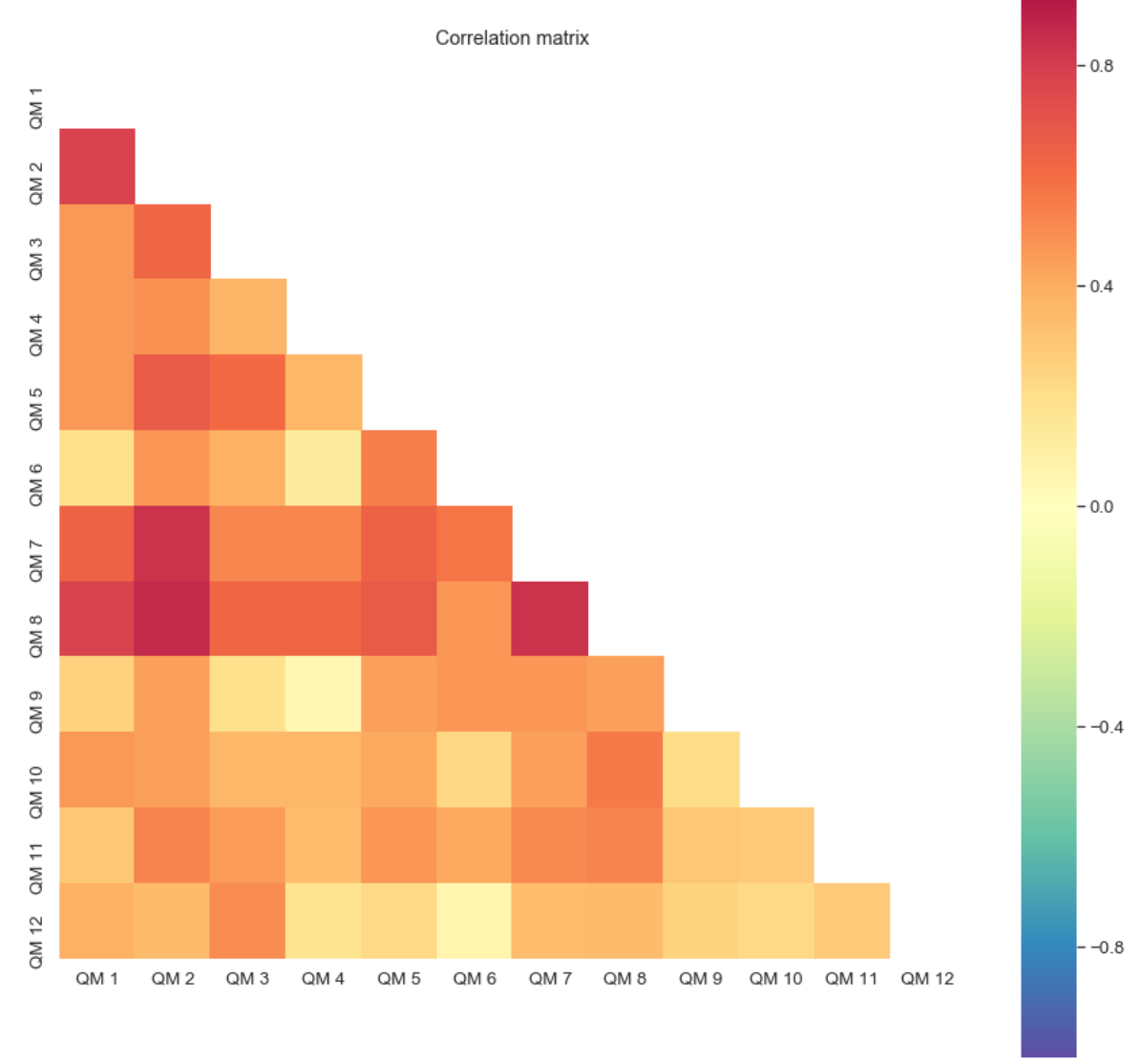

Figure 69: Correlation matrix for Quality Management factors 
The table of descriptive statistics below shows the top competency factors contributing to the PPA score, the coefficient of the correlation, and the p-value.

Table 47: Correlation of Quality Management factors vs PPA score

\begin{tabular}{|l|l|c|c|}
\hline Code & Factor & $\begin{array}{c}\text { Correlation } \\
\text { to PPA }\end{array}$ & P-value \\
\hline QM 3 & $\begin{array}{l}\text { Quality control plan performed on procured items, fabrication, and } \\
\text { construction }\end{array}$ & 0.292 & 0.027 \\
\hline QM 5 & Quality control is plan communicated among the project team & 0.273 & 0.040 \\
\hline QM 6 & people, tools, systems in place to perform the quality control plan & 0.331 & 0.012 \\
\hline QM 7 & Quality inspection procedures are well defined & 0.308 & 0.020 \\
\hline
\end{tabular}

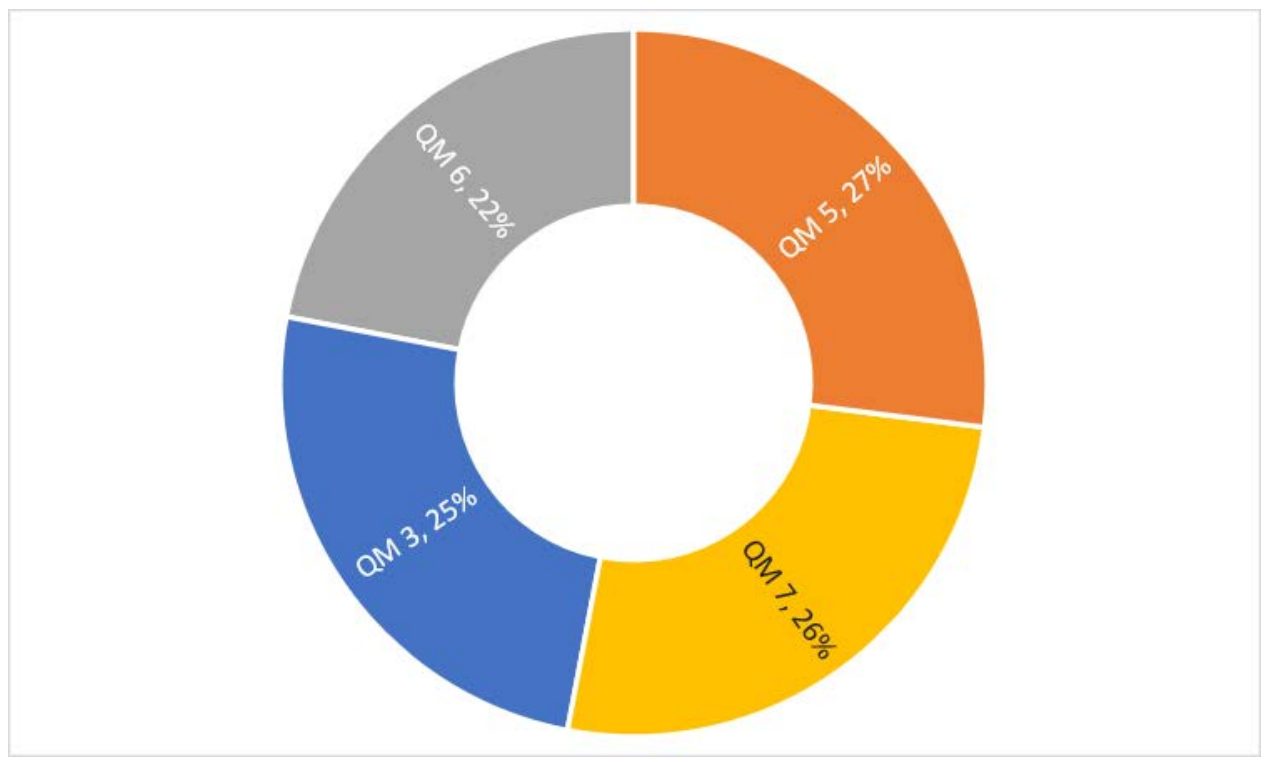

Figure 70: Weights of subcategories under Quality Management factors

A simplified equation was developed to accommodate the unstandardized data. The aggregation of this transformation for the Quality Management factors resulted in the constant term. The results of the Quality Management score are in a standardized form, i.e. a value that can be positive or negative. For better visualization and comparison of projects to one another, the authors scaled the generated Quality Management score to have a range between 5\% to 95\%. A 
range of $[5,95 \%]$ was chosen instead of $[0,100 \%]$, as it is not reasonable to presume the worst possible project performance to be $0 \%$ and the best possible project performance to be $100 \%$. Therefore, the equation below eliminates errors that stem from standardization, while it also provides a user-friendly methodology to compute the Quality Management score of a range between $5 \%$ to $95 \%$ :

$$
Q M \text { Score }=0.050+0.253 Q M 3+0.224 Q M 5+0.198 Q M 6+0.226 Q M 7
$$

A Q-Q plot and Shapiro-Wilk test are conducted to assess the normality assumption. The following Q-Q plot shows a clear departure from normality at the overall level of the dataset.
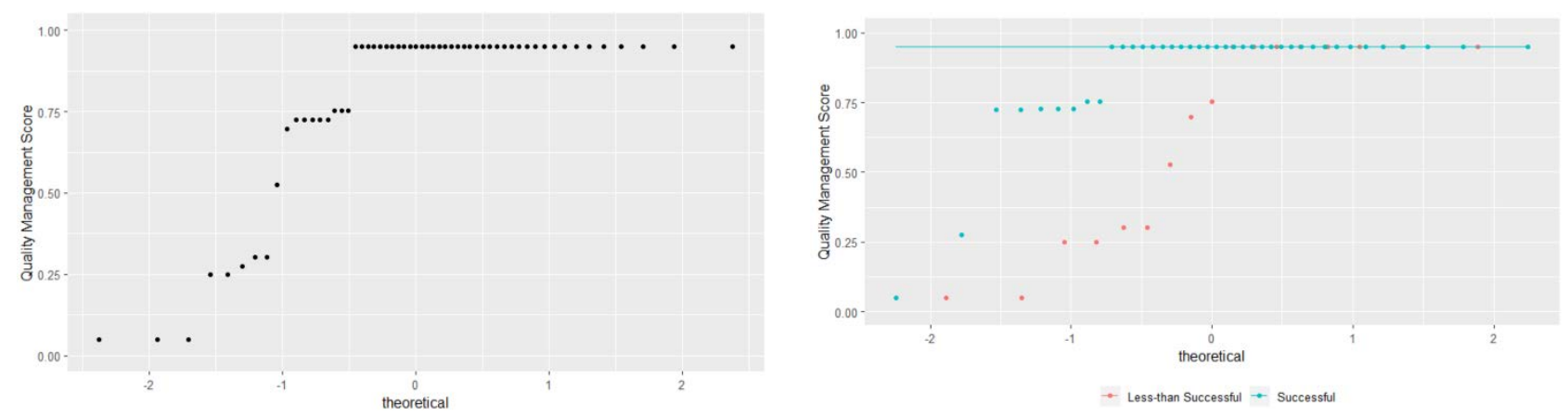

Figure 71: Q-Q plot of Quality Management score

As with the other analysis, the normality assumption is not valid, as it was confirmed at a 95\% confidence level by the Shapiro-Wilk test, which is shown in the table below.

Table 48: Shapiro-Wilk test for Quality Management score

\begin{tabular}{ccc}
\hline \hline Parameter & $p$-value & Significance at 95\% Confidence Level \\
\hline \hline Overall & 0.000 & Significant \\
Successful Projects & 0.000 & Significant \\
Less-than-successful Projects & 0.002 & Significant \\
\hline \hline
\end{tabular}


The differences between successful and less-than-successful projects are shown by using box-and-whisker plots. It is visually evident that less-than-successful projects have higher variability than successful ones, as it was illustrated from the level of compaction of the boxplot. That was also evident from the Fligner-Killeen test returning a p-value of 0.000 , which rejects the null hypothesis of equal variance among project status, confirming the previous suggestion of higher variability among less-than-successful projects at a 95\% confidence level. The Wilcoxon rank-sum test returned a p-value of 0.008 , which demonstrates that the data provides evidence to reject the null hypothesis at a 5\% significance level, thus concluding at a 95\% confidence level that there is statistically significant evidence that Quality Management score varies across the status of the project. Thus, it shows that successful projects usually focus on having an effective quality management plan contrary to less-than-successful projects, which might not give it enough attention.

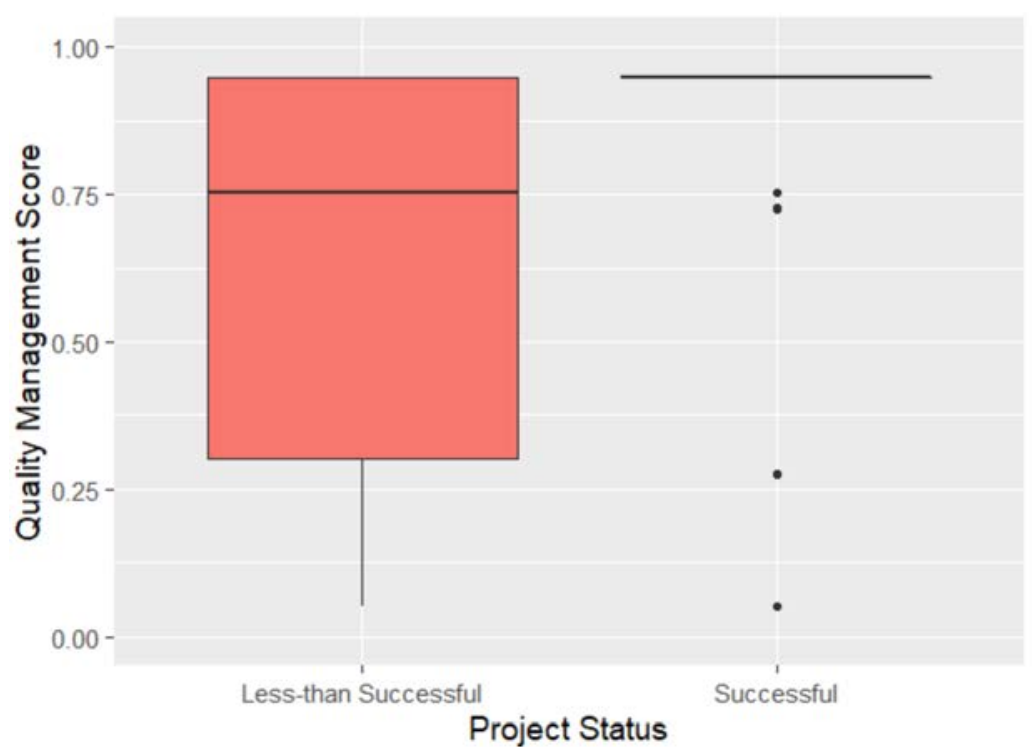

Figure 72: Boxplot of Quality Management score 
The table of descriptive statistics below shows that the median/mean for Quality Management score for successful projects (95.0/87.3\%) is higher than less-than-successful projects (75.2/53.4\%), which shows the importance of having an effective quality management plan for project success.

Table 49: Descriptive statistics for Quality Management score

\begin{tabular}{ccc}
\hline \hline Statistical Test & Successful Projects & Less-than-successful Projects \\
\hline Mean & $87.3 \%$ & $63.4 \%$ \\
Maximum & $95.0 \%$ & $95.0 \%$ \\
75\% Quantile & $95.0 \%$ & $95.0 \%$ \\
50\% Quantile & $95.0 \%$ & $75.2 \%$ \\
25\% Quantile & $95.0 \%$ & $30.3 \%$ \\
Minimum & $5.0 \%$ & $5.0 \%$ \\
\hline \hline
\end{tabular}

\subsubsection{Labor Productivity}

The figure below shows the pairwise correlation matrix for labor productivity factors. These results show that there is no occurrence of high intercorrelations among independent variables in accordance with the cutoff value. 


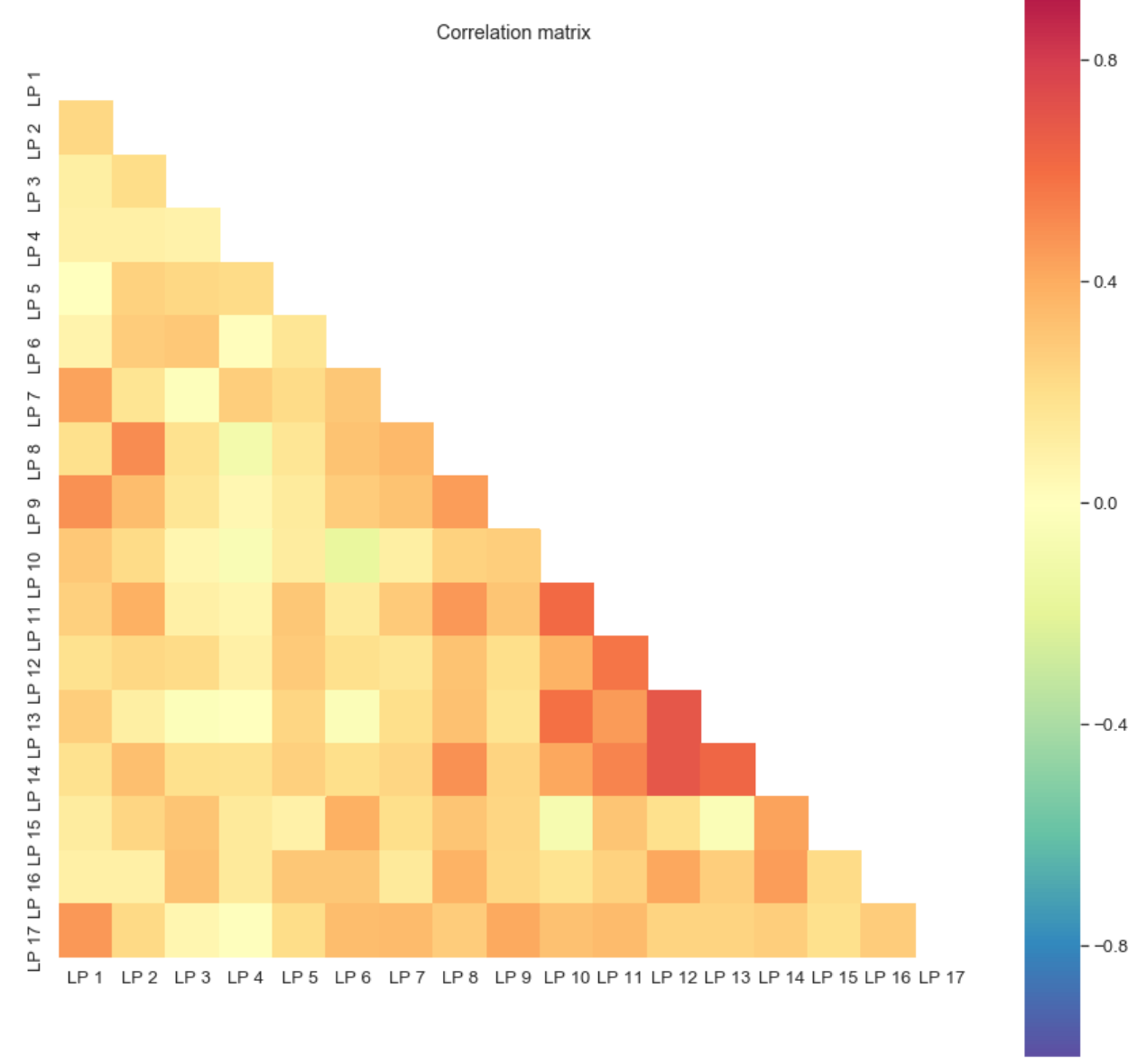

Figure 73: Correlation matrix for Labor Productivity factors

The table of descriptive statistics below shows the top competency factors contributing to the PPA score, the coefficient of the correlation, and the p-value.

Table 21: Correlation of Labor Productivity factors vs PPA score

\begin{tabular}{|l|l|c|c|}
\hline Code & Factor & Correlation to PPA & P-value \\
\hline LP 2 & A plan to secure and retain skilled labor is in place & 0.293 & 0.027 \\
\hline LP 6 & A craft evaluation/qualification program is in place & 0.270 & 0,014 \\
\hline
\end{tabular}




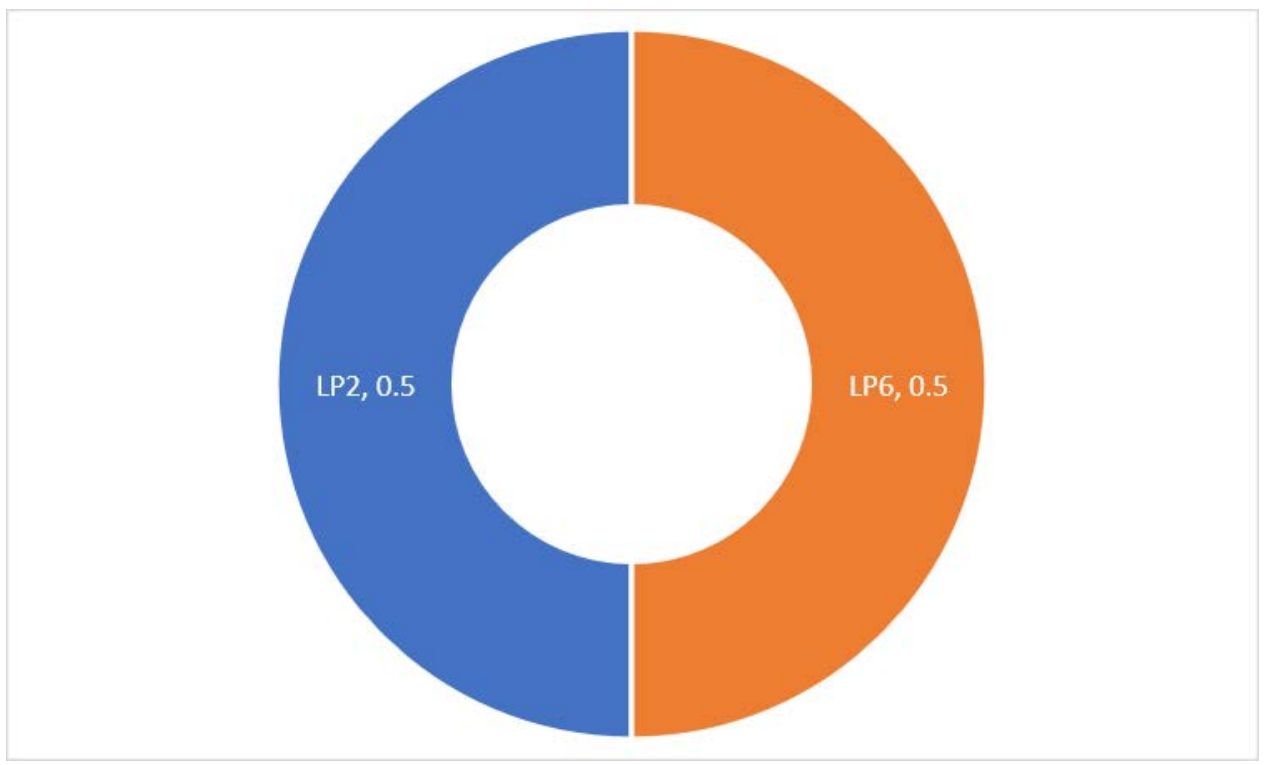

Figure 22: Weights of Subcategories under Labor Productivity factors

A simplified equation was developed to accommodate the unstandardized data. The aggregation of this transformation for the Labor Productivity factors resulted in the constant term. The results of the Labor Productivity score are in a standardized form, i.e. a value that can be positive or negative. For better visualization and comparison of projects to one another, the authors scaled the generated Labor Productivity score to have a range between 5\% to 95\%. A range of [5, 95\%] was chosen instead of [0,100\%], as it is not reasonable to presume the worst possible project performance to be $0 \%$ and the best possible project performance to be $100 \%$. Therefore, the equation below eliminates errors that stem from standardization, while it also provides a userfriendly methodology to compute the Labor Productivity score of a range between 5\% to 95\%:

$$
L P \text { Score }=0.050+0.593 L P 2+0.407 L P 6
$$

A Q-Q plot and Shapiro-Wilk test are conducted to assess the normality assumption. The following Q-Q plot shows a clear departure from normality at the overall level of the dataset. 

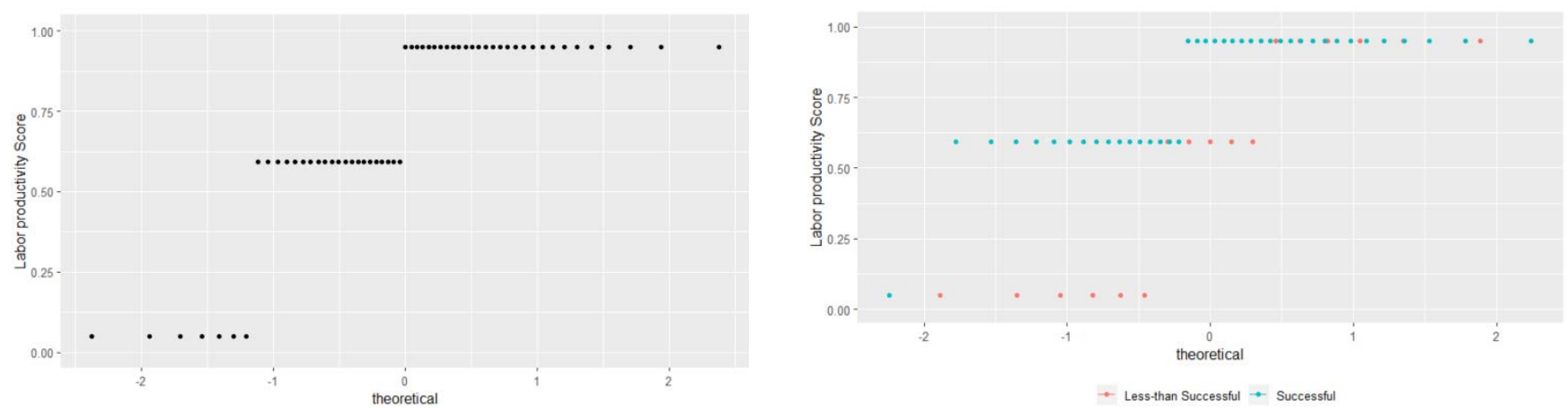

Figure 74: Q-Q Plot of Labor Productivity score

As with the other analysis, the normality assumption is not valid, as it was confirmed at a 95\% confidence level by the Shapiro-Wilk test, which is shown in the table below.

Table 17: Shapiro-Wilk Test for Labor Productivity score

\begin{tabular}{ccc}
\hline \hline Parameter & $p$-value & Significance at 95\% confidence level \\
\hline \hline Overall & 0.000 & Significant \\
Successful projects & 0.000 & Significant \\
Less-than-Successful projects & 0.001 & Significant \\
\hline \hline
\end{tabular}

The differences between successful and less-than-successful projects are shown by using box-and-whisker plots. It is visually evident that less-than-successful projects have higher variability than successful ones, as it was illustrated from the level of compaction of the boxplot. That was also evident from the Fligner-Killeen test returning a p-value of 0.005 , which rejects the null hypothesis of equal variance among project status, confirming the previous suggestion of 
higher variability among less-than-successful projects at a 95\% confidence level. The Wilcoxon rank-sum test returned a p-value of 0.019 , which demonstrates that the data provides evidence to reject the null hypothesis at a 5\% significance level, thus concluding at a 95\% confidence level that there is statistically significant evidence that Labor Productivity score varies across the status of the project. Thus, it shows that successful projects usually focus on tracking the productivity labor force contrary to less-than-successful projects, which might not give it enough attention.

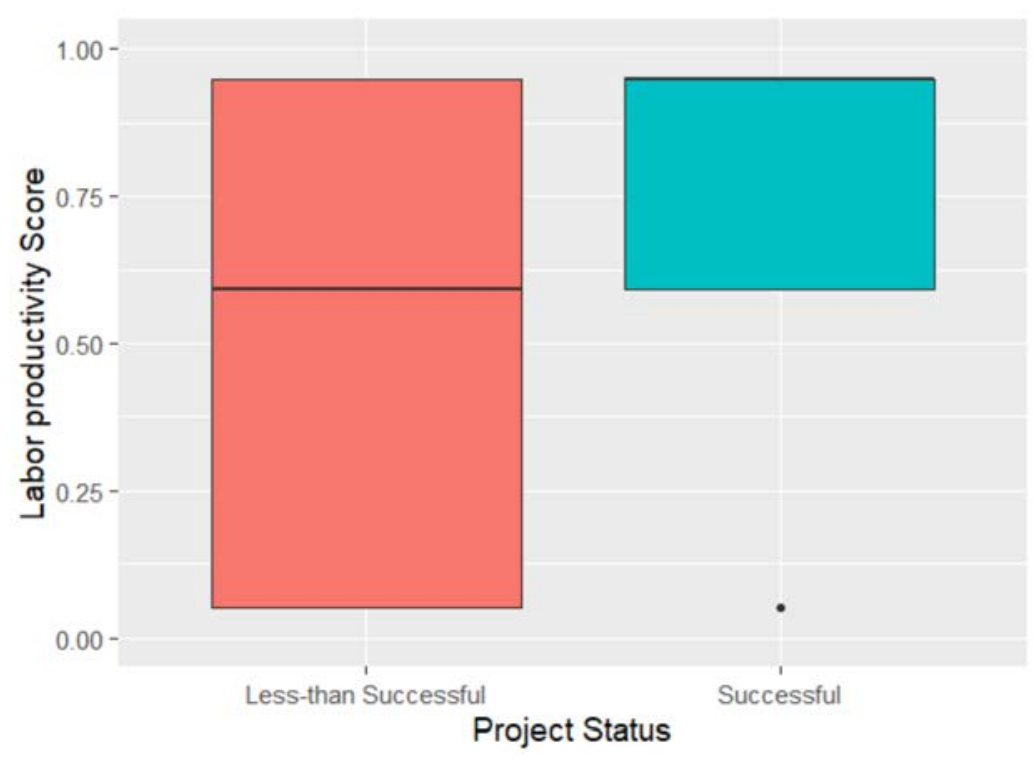

Figure 75: Boxplot of Labor Productivity score

The table of descriptive statistics below shows that the median/mean for Labor Productivity score for successful projects (95.0/78.5\%) is higher than less-than-successful projects (59.3/52.7\%), which shows the importance of tracking the productivity labor force for project success. 
Table 50: Descriptive statistics for Labor Productivity score

\begin{tabular}{ccc}
\hline \hline Statistical Test & Successful Projects & Less-than-successful Projects \\
\hline \hline Mean & $78.5 \%$ & $52.7 \%$ \\
Maximum & $95.0 \%$ & $95.0 \%$ \\
$75 \%$ Quantile & $95.0 \%$ & $95.0 \%$ \\
$50 \%$ Quantile & $95.0 \%$ & $59.3 \%$ \\
$25 \%$ Quantile & $59.3 \%$ & $5.0 \%$ \\
Minimum & $5.0 \%$ & $5.0 \%$ \\
\hline \hline
\end{tabular}

\subsubsection{Execution \& Commissioning}

The figure below shows the pairwise correlation matrix for Execution \& Commissioning factors. These results show there is a high occurrence of intercorrelations among E\&C 3, 4, 6, and 7 with a coefficient exceeding 0.75 , which illustrates that it is necessary to have a well-defined construction execution plan and what is more important is that this plan should be aligned with the project execution plan. Moreover, contractors should be familiar with their obligations and all project teams should be also familiar with these obligations, thus these factors are highly correlated to each other. Then, a correlation test is conducted to analyze the highly correlated factors with the PPA score to determine which factor will be considered in the mode; otherwise, these factors will not be considered. 


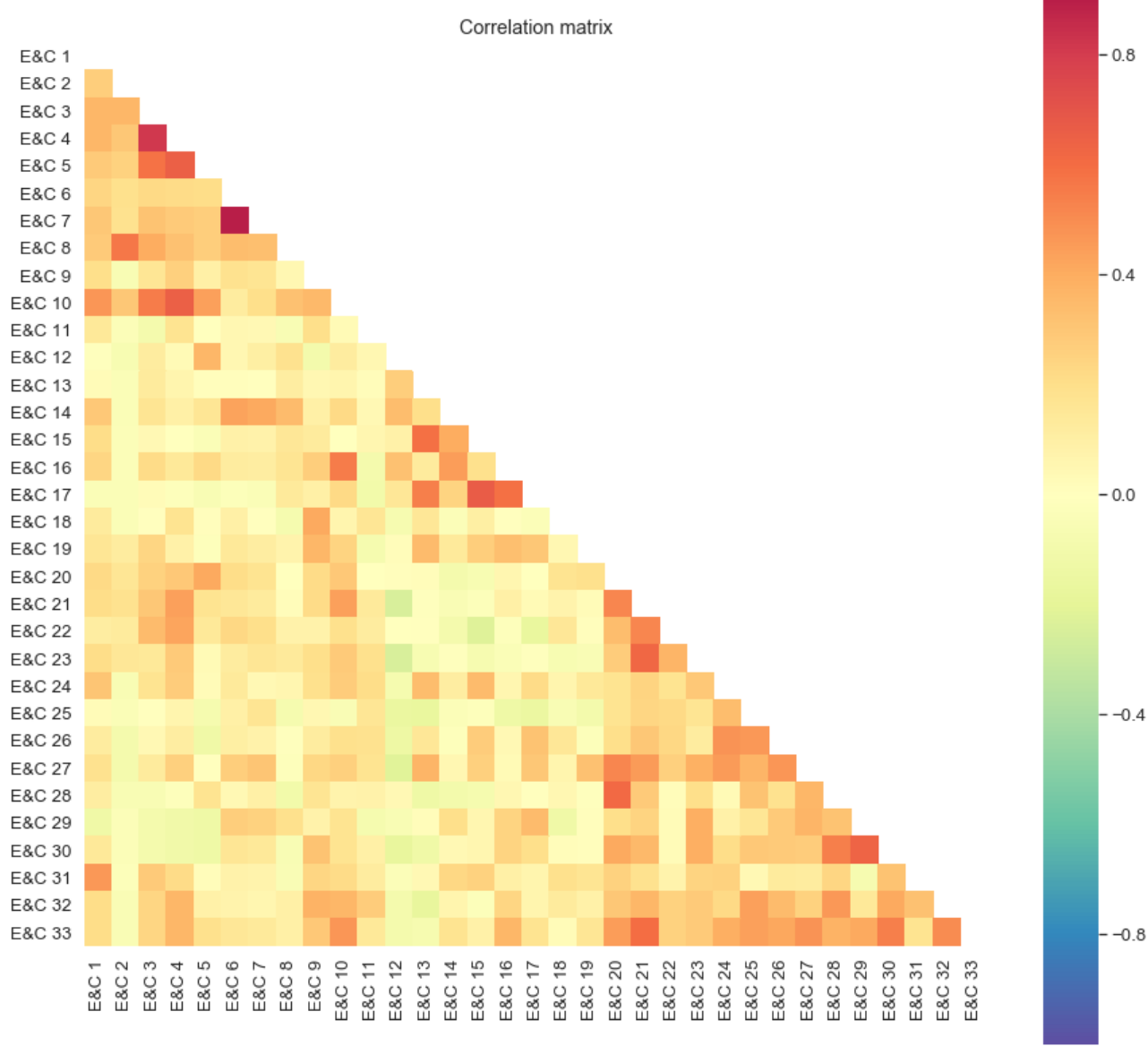

Figure 76: Correlation matrix for Execution \& Commissioning factors

The table of descriptive statistics below shows the top competency factors contributing to the PPA score, the coefficient of the correlation and the p-value.

Table 51: Correlation of Execution \& Commissioning factors vs PPA score

\begin{tabular}{|l|l|c|c|}
\hline Code & Factor & $\begin{array}{c}\text { Correlation } \\
\text { to PPA }\end{array}$ & P-value \\
\hline E\&C 3 & Construction execution plan is well defined & 0.289 & 0.029 \\
\hline E\&C 10 & A coordination plan is well defined between all project parties & 0.425 & 0.001 \\
\hline E\&C 19 & A plan to minimize out-of-sequence work is well defined & 0.402 & 0.002 \\
\hline E\&C 20 & $\begin{array}{l}\text { Turnover procedures (from construction to commissioning) are well } \\
\text { defined }\end{array}$ & 0.368 & 0.005 \\
\hline E\&C 21 & Construction is aligned with commissioning and operations & 0.281 & 0.034 \\
\hline
\end{tabular}




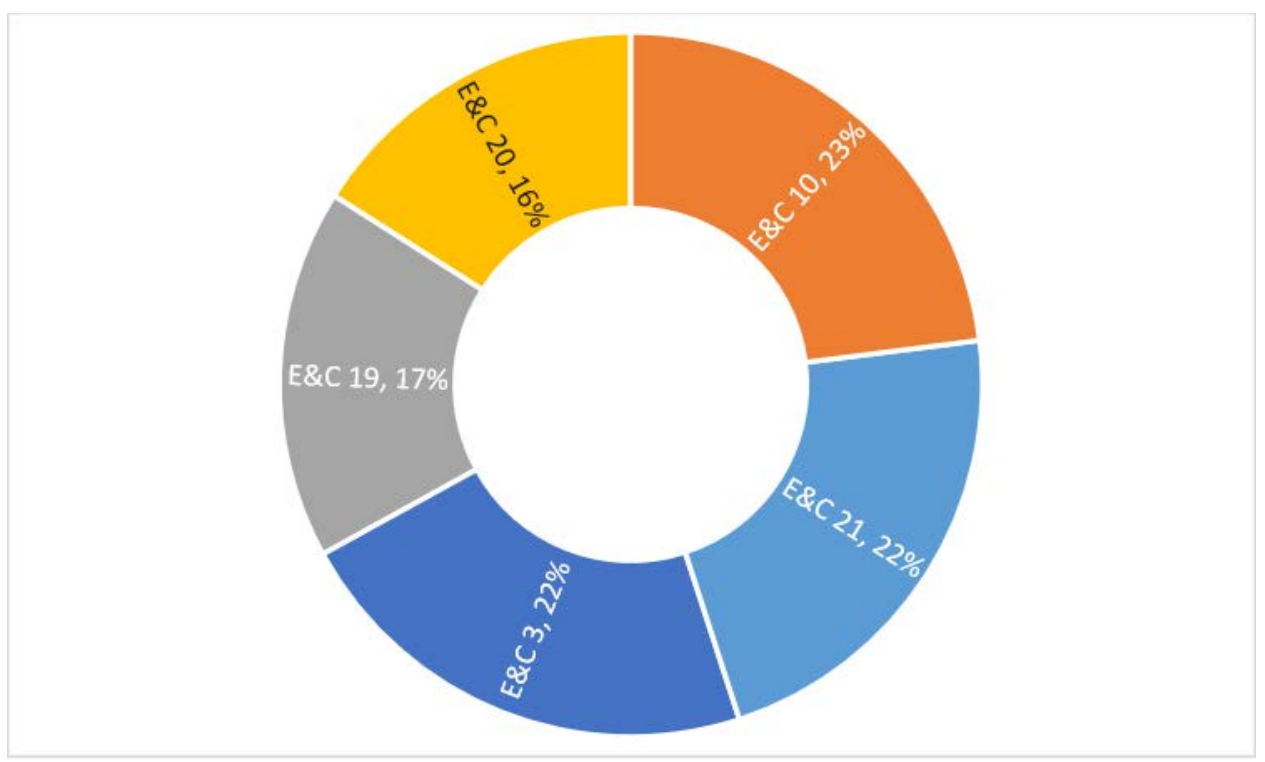

Figure 77: Weights of subcategories under Execution \& Commissioning factors

A simplified equation was developed to accommodate the unstandardized data. The aggregation of this transformation for the Execution \& Commissioning factors resulted in the constant term. The results of the Execution \& Commissioning score are in a standardized form, i.e. a value that can be positive or negative. For better visualization and comparison of projects to one another, the authors scaled the generated Execution \& Commissioning score to have a range between $5 \%$ to $95 \%$. A range of $[5,95 \%$ ] was chosen instead of $[0,100 \%]$, as it is not reasonable to presume the worst possible project performance to be $0 \%$ and the best possible project performance to be $100 \%$. Therefore, the equation below eliminates errors that stem from standardization, while it also provides a user-friendly methodology to compute the Execution \& Commissioning score of a range between $5 \%$ to $95 \%$ :

$$
\begin{gathered}
E \& C \text { Score }=0.050+0.224 E \& C 3+0.236 E \& C 10+0.152 E \& C 19+0.115 E \& C 20+ \\
0.173 E \& C 21
\end{gathered}
$$


A Q-Q plot and Shapiro-Wilk test are conducted to assess the normality assumption. The following Q-Q plot shows a clear departure from normality at the overall level of the dataset.
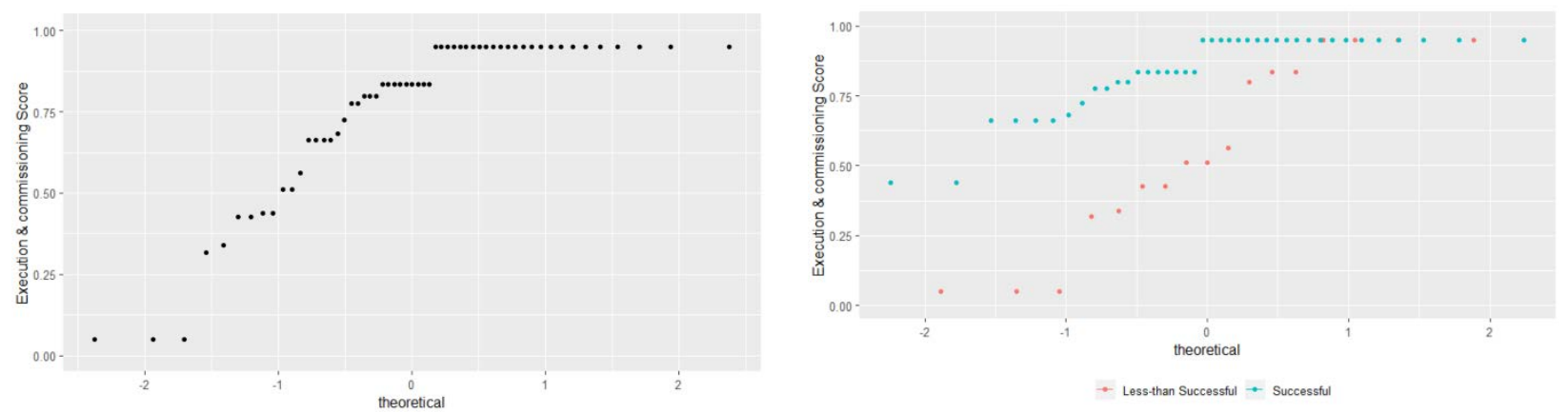

Figure 78: Q-Q plot of Execution \& Commissioning score

As with the other analysis, the normality assumption is not valid, as it was confirmed at a 95\% confidence level by the Shapiro-Wilk test, which is shown in the table below.

Table 52: Shapiro-Wilk test for Execution \& Commissioning score

Parameter

$p$-value

Significance at 95\% Confidence Level

\begin{tabular}{ccc}
\hline \hline Overall & 0.000 & Significant \\
Successful Projects & 0.000 & Significant \\
Less-than-successful Projects & 0.044 & Significant \\
\hline \hline
\end{tabular}

The differences between successful and less-than-successful projects are shown by using box-and-whisker plots. It is visually evident that less-than-successful projects have higher variability than successful ones, as it was illustrated from the level of compaction of the boxplot. That was also evident from the Fligner-Killeen test returning a p-value of 0.0012, which rejects the null hypothesis of equal variance among project status, confirming the previous suggestion of 
higher variability among less-than-successful projects at a 95\% confidence level. The Wilcoxon rank-sum test returned a p-value of 0.0014 , which demonstrates that the data provides evidence to reject the null hypothesis at a 5\% significance level, thus concluding at a 95\% confidence level that there is statistically significant evidence that Execution \& Commissioning score varies across the status of the project. Thus, it shows that successful projects usually focus on properly planning effective execution and commissioning measures, contrary to less-than-successful projects, which might not give it enough attention.

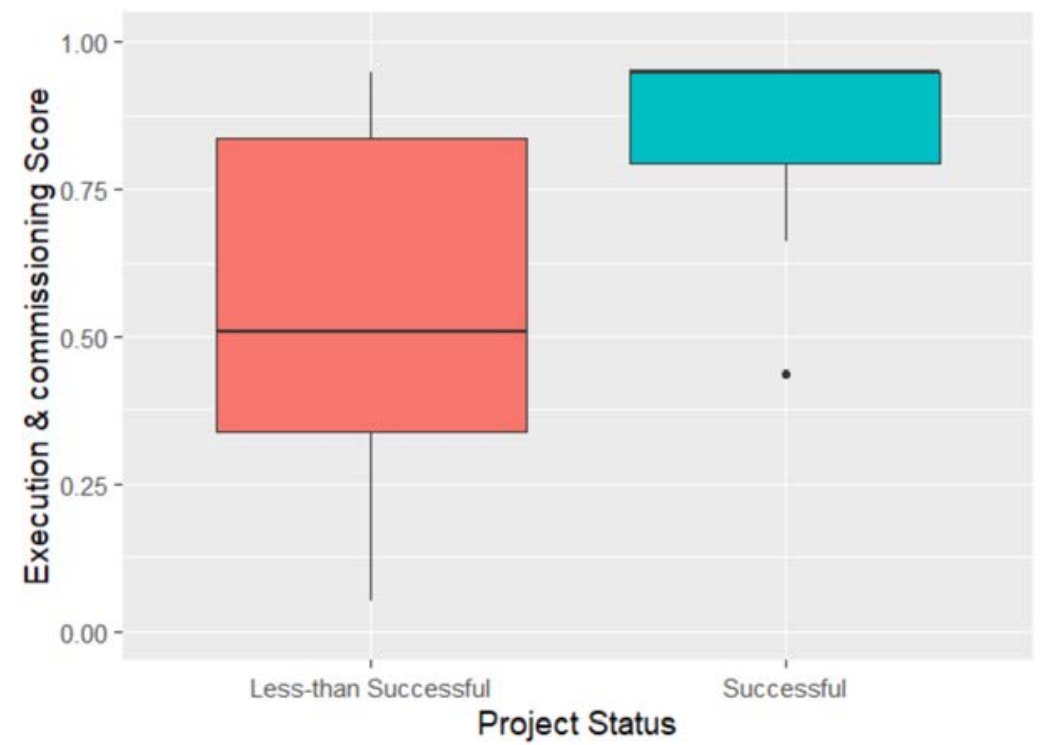

Figure 79: Boxplot of Execution \& Commissioning score

The table of descriptive statistics below shows that the median/mean for Execution \& Commissioning score for successful projects (95.0/84.7\%) is higher than less-than-successful projects (51.0/55.9\%), which shows the importance of having good measures needed for effective execution and commissioning for project success. 
Table 53: Descriptive statistics for Execution \& Commissioning score

\begin{tabular}{ccc}
\hline \hline Statistical Test & Successful Projects & Less-than-successful Projects \\
\hline \hline Mean & $84.7 \%$ & $55.9 \%$ \\
Maximum & $95.0 \%$ & $95.0 \%$ \\
$75 \%$ Quantile & $95.0 \%$ & $83.5 \%$ \\
$50 \%$ Quantile & $95.0 \%$ & $51.0 \%$ \\
25\% Quantile & $79.2 \%$ & $33.8 \%$ \\
Minimum & $43.8 \%$ & $5.0 \%$ \\
\hline \hline
\end{tabular}




\subsubsection{Change Management \& Project Control}

The figure below shows the pairwise correlation matrix for Change Management \& Project Control (CMPC) factors. These results show there is a high occurrence of intercorrelations among CMPC 16 and CMPC 17 with a coefficient 0.786, which illustrates that it is necessary to have a progress tracking plan and systems, which is highly correlated to have the resources deployed to support the progress tracking system. Then, a correlation test is conducted to analyze the highly correlated factors with the PPA score to determine which factor will be considered in the mode; otherwise, these factors will not be considered.

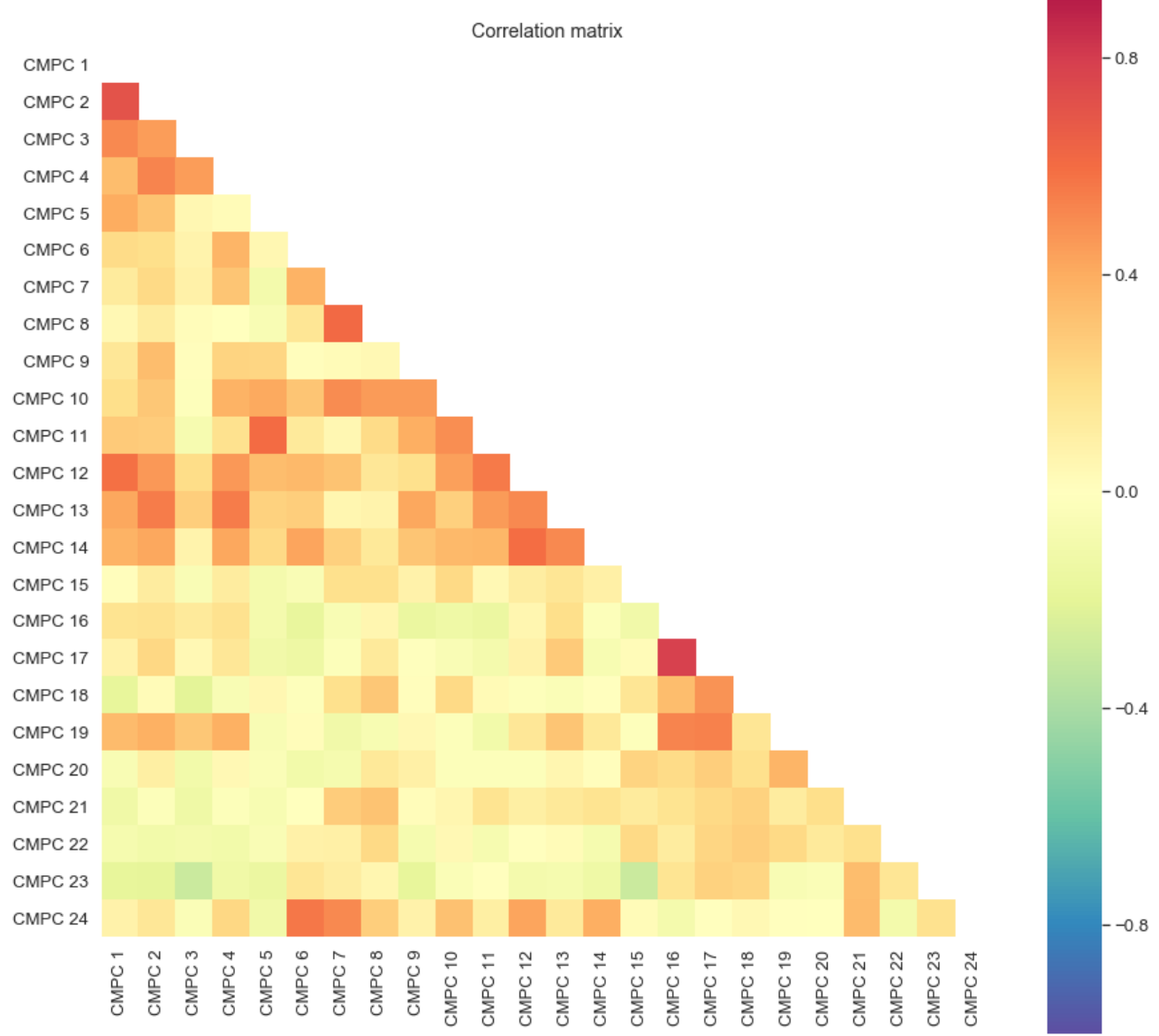

Figure 80: Correlation matrix for Change Management \& Project Control factors 
The table of descriptive statistics below shows the top competency factors contributing to the

PPA score, the coefficient of the correlation, and the p-value.

Table 54: Correlation of Change Management \& Project Control factors vs PPA score

\begin{tabular}{|l|l|c|c|}
\hline Code & Factor & $\begin{array}{c}\text { Correlation to } \\
\text { PPA }\end{array}$ & P-value \\
\hline CMPC 4 & Evaluation and quantification of changes process are well defined & 0.414 & 0.001 \\
\hline CMPC 7 & Document control of change orders are well defined & 0.420 & 0.001 \\
\hline CMPC 8 & Tracking change orders & 0.334 & 0.011 \\
\hline CMPC 9 & Payment procedures for changes are well defined & 0.426 & 0.001 \\
\hline CMPC 10 & Change orders are reconciled to scope & 0.369 & 0.005 \\
\hline CMPC 11 & $\begin{array}{l}\text { Chain of communication of change orders are well defined to project } \\
\text { parties }\end{array}$ & 0.406 & 0.002 \\
\hline CMPC 12 & $\begin{array}{l}\text { Change management roles and responsibilities are well defined to } \\
\text { project parties }\end{array}$ & 0.438 \\
\hline CMPC 14 & Turnover procedures for change management are well defined & 0.393 \\
\hline CMPC 21 & Updating schedule process is well defined & 0.286 & 0.002 \\
\hline CMPC 24 & Cost contingency management plan is well defined & 0.272 & 0.031 \\
\hline
\end{tabular}

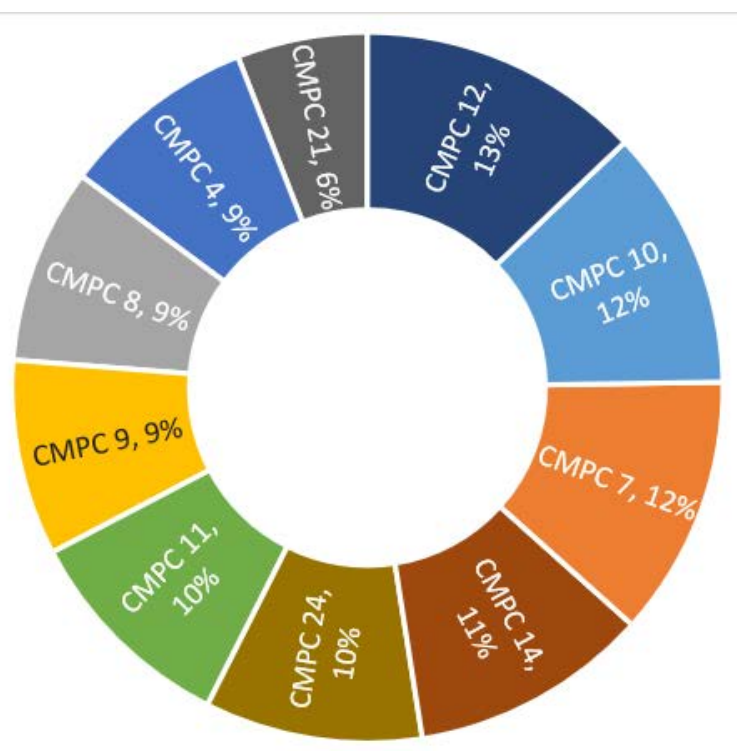

Figure 81: Weights of subcategories under Change Management \& Project Control factors

A simplified equation was developed to accommodate the unstandardized data. The aggregation of this transformation for the CMPC factors resulted in the constant term. The results of the CMPC score are in a standardized form, i.e. a value that can be positive or negative. For 
better visualization and comparison of projects to one another, the authors scaled the generated CMPC score to have a range between $5 \%$ to $95 \%$. A range of [5, 95\%] was chosen instead of [0, $100 \%]$, as it is not reasonable to presume the worst possible project performance to be $0 \%$ and the best possible project performance to be $100 \%$. Therefore, the equation below eliminates errors that stem from standardization, while it also provides a user-friendly methodology to compute the CMPC score of a range between $5 \%$ to $95 \%$ :

$$
\begin{gathered}
\text { CMPC Score }=-0.039+0.089 \text { CMPC } 4+0.120 \text { CMPC } 7+0.123 \text { CMPC } 8+ \\
0.103 \text { CMPC } 10+0.111 \text { CMPC } 11+0.106 \text { CMPC } 12+0.088 \text { CMPC } 14+ \\
0.074 \text { CMPC } 21+0.095 \text { CMPC } 24
\end{gathered}
$$

A Q-Q plot and Shapiro-Wilk test are conducted to assess the normality assumption. The following Q-Q plot shows a clear departure from normality at the overall level of the dataset.
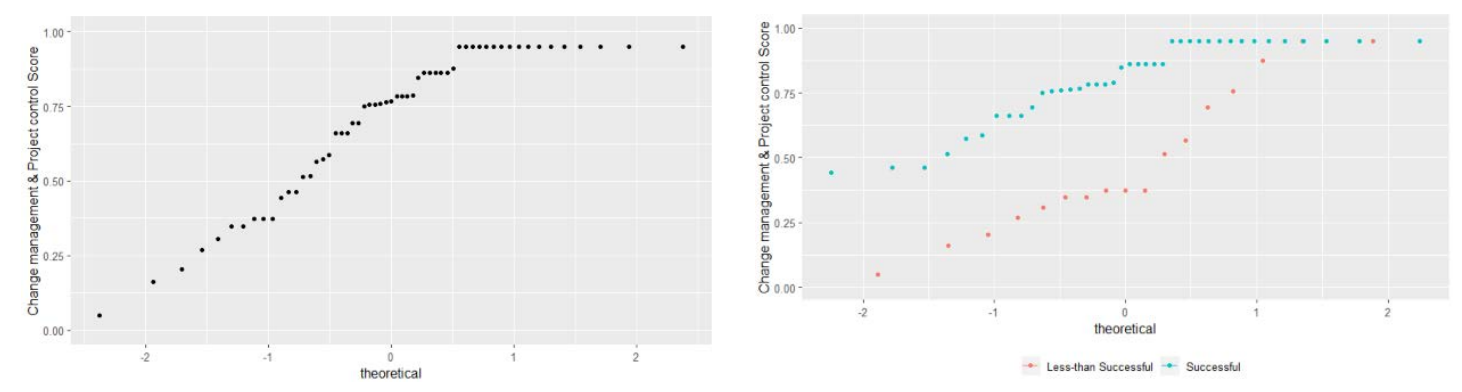

Figure 30: Q-Q plot of Change Management \& Project Control score

As with the other analysis, the normality assumption is not valid, as it was confirmed at a $95 \%$ confidence level by the Shapiro-Wilk test, which is shown in the table below. 
Table 55: Shapiro-Wilk test for Change Management \& Project Control score

\begin{tabular}{ccc}
\hline \hline Parameter & $p$-value & Significance at 95\% Confidence Level \\
& & \\
\hline \hline Overall & 0.000 & Significant \\
Successful Projects & 0.000 & Significant \\
Less-than-successful Projects & 0.1676 & Not Significant \\
\hline \hline
\end{tabular}

The differences between successful and less-than-successful projects are shown by using box-and-whisker plots. It is visually evident that successful and less-than-successful projects have similar variability to each other, as it was illustrated from the level of compaction of the boxplot and that was evident from the Fligner-Killeen test returning a p-value of 0.08 , confirming the equal variance of both project statuses at a 95\% confidence level. The Wilcoxon rank-sum test returned a p-value of 0.000, which demonstrates that the data provides evidence to reject the null hypothesis at a $5 \%$ significance level, thus concluding at a $95 \%$ confidence level that there is statistically significant evidence that CMPC score varies across the status of the project. Thus, it shows that successful projects usually focus on having an effective change management plan and a successful progress monitoring plan throughout a project’s lifecycle, contrary to less-than-successful projects, which might not give these plans enough attention. 


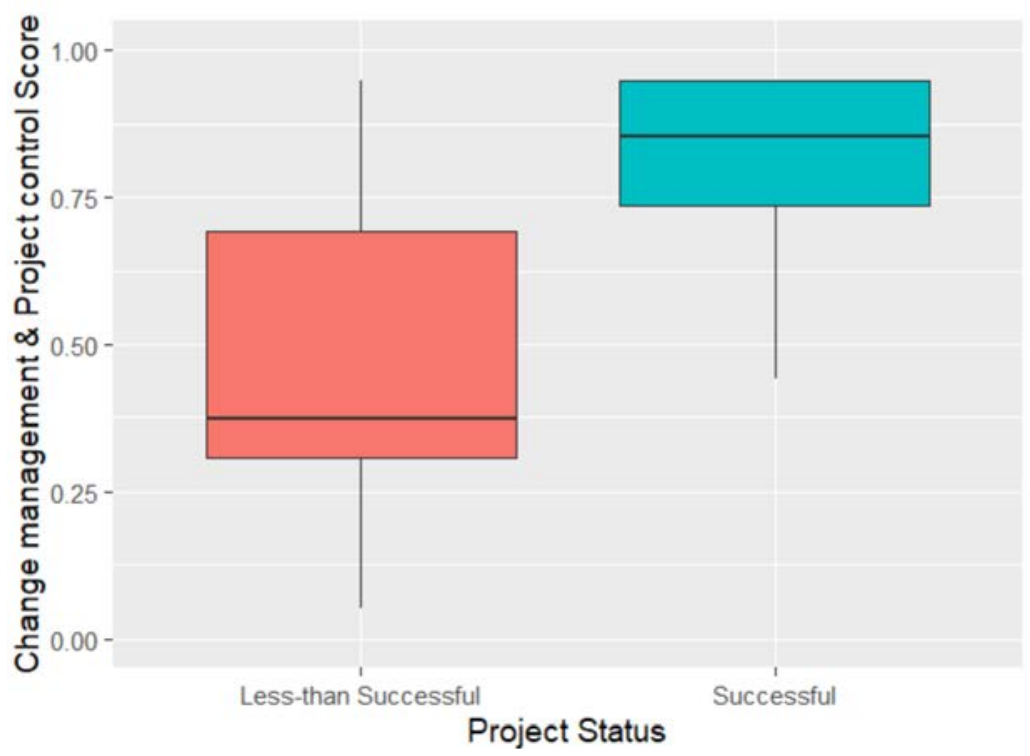

Figure 82: Boxplot of Change Management \& Project Control score

The table of descriptive statistics below shows that the median/mean for Execution \& Commissioning score for successful projects (85.5/80.2\%) is higher than less-than-successful projects (37.3/47.7\%), which shows the importance of having an effective change management plan and a successful progress monitoring plan throughout a project's lifecycle for project success Table 56: Descriptive statistics for Change Management \& Project Control score

\begin{tabular}{ccc}
\hline \hline Statistical Test & Successful Projects & Less-than-successful Projects \\
\hline \hline Mean & $80.2 \%$ & $47.7 \%$ \\
Maximum & $95.0 \%$ & $95.0 \%$ \\
$75 \%$ Quantile & $95.0 \%$ & $69.4 \%$ \\
$50 \%$ Quantile & $85.5 \%$ & $37.3 \%$ \\
$25 \%$ Quantile & $73.7 \%$ & $30.7 \%$ \\
Minimum & $44.4 \%$ & $5.0 \%$ \\
\hline \hline
\end{tabular}




\subsection{Correlation Matrix between Competency Factors and Performance Metrics}

In this section, the impact of the eight competency categories on project performance is investigated by statically examining the correlations between each competency category with each performance metric to form the $8 \times 9$ matrix as shown in Table 57. Spearman's Correlation test is conducted, such that the null hypothesis states that there is no correlation between the competency factor and performance metric, while the alternative hypothesis states that correlation exists. Using Spearman's Correlation test for hypothesis testing produces a $p$-value, which can be thought of as the probability of having no statistical correlation between the competency factor and performance metric. Accordingly, a p-value of less than 0.05 demonstrates that the data provides evidence to reject the null hypothesis at a 5\% significance level, thus concluding at a 95\% confidence level that there is a statistically significant correlation between the competency factor and performance metric. A second statistical metric is produced from this analysis is the Spearman's Rho correlation coefficient, which measures the relationship between each of the competency factor and performance metric, so that it has a value of between -1 and 1 . A 0 means there is no relationship between the variables at all, while -1 or 1 means that there is a perfect negative or positive correlation. In Table 57, the key to the color-coding is as follows: the gray color means that the correlation is not significant, the corresponding black color means that the p-value is greater than 0.05 , the yellow color means that the correlation is significant, and the corresponding green color means that the $p$-value is less than 0.05 . 
Table 57: Correlation matrix between competency factors and performance metrics

\begin{tabular}{|c|c|c|c|c|c|c|c|c|c|}
\hline & & Construction Cost Growth & Percent Delta & Construction Schedule Growth & Lost Time Injuries & Rework Percentage & Value of Punch-list Items & \#RFIs/\$M & RFI Processing Time \\
\hline \multirow{2}{*}{ AL\&T score } & Cor. & -0.376 & -0.173 & -0.333 & -0.308 & -0.005 & -0.238 & -0.037 & -0.212 \\
\hline & $P$-Value & 0.001 & 0.132 & 0.003 & 0.006 & 0.967 & 0.037 & 0.749 & 0.065 \\
\hline \multirow{2}{*}{ PL\&D score } & Cor. & -0.374 & -0.21 & -0.548 & -0.271 & -0.193 & -0.287 & -0.065 & -0.181 \\
\hline & $P$-Value & 0.001 & 0.067 & 0 & 0.017 & 0.093 & 0.011 & 0.573 & 0.114 \\
\hline \multirow{2}{*}{ E\&C score } & Cor. & -0.369 & -0.08 & -0.248 & -0.284 & -0.18 & -0.084 & -0.078 & -0.423 \\
\hline & $P$-Value & 0.001 & 0.489 & 0.03 & 0.012 & 0.118 & 0.468 & 0.498 & 0 \\
\hline \multirow{2}{*}{ Proc score } & Cor. & -0.235 & -0.18 & -0.342 & -0.192 & -0.037 & -0.316 & -0.012 & -0.262 \\
\hline & $P$-Value & 0.04 & 0.117 & 0.002 & 0.094 & 0.746 & 0.005 & 0.916 & 0.021 \\
\hline \multirow{2}{*}{ QM score } & Cor. & -0.069 & -0.111 & -0.339 & -0.378 & -0.071 & -0.223 & -0.049 & -0.281 \\
\hline & $P$-Value & 0.552 & 0.335 & 0.003 & 0.001 & 0.538 & 0.051 & 0.672 & 0.013 \\
\hline \multirow{2}{*}{ CMPC score } & Cor. & -0.385 & -0.218 & -0.458 & -0.347 & -0.012 & -0.278 & -0.019 & -0.241 \\
\hline & $P$-Value & 0.001 & 0.056 & 0 & 0.002 & 0.92 & 0.014 & 0.872 & 0.035 \\
\hline \multirow{2}{*}{ CNRM score } & Cor. & -0.097 & -0.126 & -0.352 & -0.157 & -0.072 & -0.238 & -0.085 & -0.314 \\
\hline & $P$-Value & 0.399 & 0.274 & 0.002 & 0.174 & 0.532 & 0.037 & 0.465 & 0.005 \\
\hline \multirow{2}{*}{ LP score } & Cor. & -0.184 & -0.168 & -0.267 & -0.323 & -0.098 & -0.136 & -0.008 & -0.12 \\
\hline & $P$-Value & 0.109 & 0.145 & 0.019 & 0.004 & 0.397 & 0.237 & 0.947 & 0.299 \\
\hline
\end{tabular}




\subsection{Summary and Conclusions}

This chapter discusses the development of the mathematical formulation of the competency category-specific scores for each of the eight categories: alignment \& team integration, planning \& design, procurement management, risk management, quality management, labor productivity, execution \& commissioning, and change management \& project control. Finally, the researcher assessed the overall effect of increased each competency category-specific score on the likelihood of the project being successful or less-than-successful projects and concluded that there is statistically significant evidence that all the competency categories vary across the status of the project at a 95\% confidence level. 


\section{Chapter 6 Relationship between Project Competency and Project Performance}

There are no research studies that adequately studied the relationship between the project competencies as leading indicators for project performance improvement. Recognizing this gap, Chapter 6 discusses the development of the mathematical formulation of the Competency Category Assessment (CCA) score. The CCA score incorporates a set of competency category-specific scores (Chapter 5) with varying degrees of impact (i.e. weights) on project performance. The objectives of this chapter are to: 1) identify objective data-based weights for the competency categories; and therefore, alleviate the subjectivity associated with experts' judgment regarding the categories relative importance to one another; 2) quantitatively assess the relative contribution of the different competency categories to the PPA score (Chapter 4) and provide one unique comprehensive score named the CCA score; and 3) use the CCA score to predict the PPA score of projects.

\subsection{Second Mathematical Model Formulation}

Iskandar et al. (2019) have derived the second mathematical model, which is used to assess project success when compared with Overhead and Profit (OH\&P). In this study, OH\&P was categorized as a binary variable, where any project with OH\&P that was less than a certain threshold was considered average, and any project with OH\&P that was higher than this threshold was considered exceptional. The objective of this model is to derive the following linear weighted function of the standardized factors of the projects:

$$
S\left(z_{1}, z_{2}, \ldots, z_{n}\right)=w_{1} z_{1}+w_{2} z_{2}+\cdots+w_{n} z_{n}
$$

as to maximize the ratio between the length of the vector, whose components are the difference between the $S$-scores of all possible pairs of average and exceptional projects and the length of the 
vector of weights. In this dissertation, the above model is modified to be able to derive the relative contributions of the different competency categories to the (non-binary) PPA score.

Similar to the first mathematical model, the original factors are standardized in equation (1) so that they have mean 0 and standard deviation 1 . This standardization is done for each competency category score. The PPA scores of the projects are standardized to get the value $b_{j}$ for each project $j$. Again, the competency score is assumed to be given by a linear weighted function, as in equation (2), where the weights $w_{i}$ are selected in such a way as to maximize the length of the vector; and whose components are the correlations between the overall score vector, with the restriction on the weights given by equation (3).

$$
\begin{gathered}
z_{i j}=\frac{x_{i j}-\bar{x}_{i}}{ \pm s_{i}}, \\
S\left(z_{1}, z_{2}, \ldots, z_{n}\right)=w_{1} z_{1}+w_{2} z_{2}+\cdots+w_{n} z_{n} \\
\text { subject to: } \sum_{i=1}^{n} w_{i}=1
\end{gathered}
$$

All possible pairs of projects $j$ and $k$ are considered, (there are $M=m^{2}$ of these pairs). These pairs are ordered in any way that maps the set of pairs: $\{(j, k) \mid 1 \leq j \leq m, 1 \leq k \leq m\}$ in a one-to-one correspondence to the set of positive integers: $\{p \mid 1 \leq p \leq M\}$. For each such pair $p=p(j, k)$ of projects, the difference between their $S$-scores is given by:

$$
\Delta_{p}=S\left(Z_{k}\right)-S\left(Z_{j}\right)=\sum_{i=1}^{n} w_{i} z_{i k}-\sum_{i=1}^{n} w_{i} z_{i j}=\sum_{i=1}^{n} w_{i}\left(z_{i k}-z_{i j}\right)=\sum_{i=1}^{n} w_{i} \delta_{i p}
$$

where $\delta_{i p}=z_{i k}-z_{i j}$ with $p \leftrightarrow(i, j)$ being the one-to-one correspondence mentioned above.

The difference $\Delta_{p}$ should have the same sign as the difference between the standardized PPA scores $\beta_{p}=b_{k}-b_{j}$ of the corresponding pair of projects. Thus, the length of the following vector is given as follows: 


$$
\left[\begin{array}{c}
\Delta_{1} \beta_{1} \\
\Delta_{2} \beta_{2} \\
\vdots \\
\Delta_{M} \beta_{M}
\end{array}\right]=\left[\begin{array}{c}
\delta_{11} \beta_{1} w_{1}+\delta_{21} \beta_{1} w_{2}+\cdots+\delta_{n 1} \beta_{1} w_{n} \\
\delta_{12} \beta_{2} w_{1}+\delta_{22} \beta_{2} w_{2}+\cdots+\delta_{n 2} \beta_{2} w_{n} \\
\vdots \\
\delta_{1 M} \beta_{M} w_{1}+\delta_{2 M} \beta_{M} w_{2}+\cdots+\delta_{n M} \beta_{M} w_{n}
\end{array}\right]=D W
$$

where $W=\left[\begin{array}{c}w_{1} \\ w_{2} \\ \vdots \\ w_{n}\end{array}\right]$ is the $n \times 1$ weight vector, and $D=\left[\begin{array}{cccc}\delta_{11} \beta_{1} & \delta_{21} \beta_{1} & \cdots & \delta_{n 1} \beta_{1} \\ \delta_{12} \beta_{2} & \delta_{22} \beta_{2} & \cdots & \delta_{n 2} \beta_{2} \\ \vdots & \vdots & \ddots & \vdots \\ \delta_{1 M} \beta_{M} & \delta_{2 M} \beta_{M} & \cdots & \delta_{n M} \beta_{M}\end{array}\right]$, an $M \times n$ matrix.

Next, the objective is to maximize the ratio $\|D W\| /\|W\|$, or equivalently, maximize its square:

$$
\frac{\|D W\|^{2}}{\|W\|^{2}}=\frac{(D W)^{T} D W}{W^{T} W}=\frac{W^{T} D^{T} D W}{W^{T} W},
$$

which is a Rayleigh quotient that is maximized when $W$ is an eigenvector of the matrix $D^{T} D$ corresponding to its maximum eigenvalue. To satisfy equation (4), any such eigenvector $V$ is scaled by the scalar $1 /\left(\left[\begin{array}{llll}1 & 1 & \cdots & 1\end{array}\right] V\right)$ to get the weights:

$$
w_{i}=\frac{v_{i}}{\sum_{i=1}^{n} v_{i}}
$$

\subsection{Competency Assessment (CCA) Model Results}

Based on the second mathematical model, the final weights of the eight competency categories are identified as in Figure 82. The results show that the top two scores in competency categories are: planning \& design and change management \& project control, having weights of $18.61 \%$ and $16.37 \%$, respectively. Contrastingly, the alignment process \& team integration and quality management competency categories scores have the lowest contribution in calculating the PPA score with weights of $8.07 \%$ and $6.02 \%$, respectively. While having a competency score of low weight shows that it plays a comparatively small role in differentiating extraordinary projects from average ones, in some instances, both average and outstanding projects have a high 
competency score, which results in small differences and, in turn, a low weight. In these instances, subject competencies are essential for project success, rather distinguishing between extraordinary from average projects.

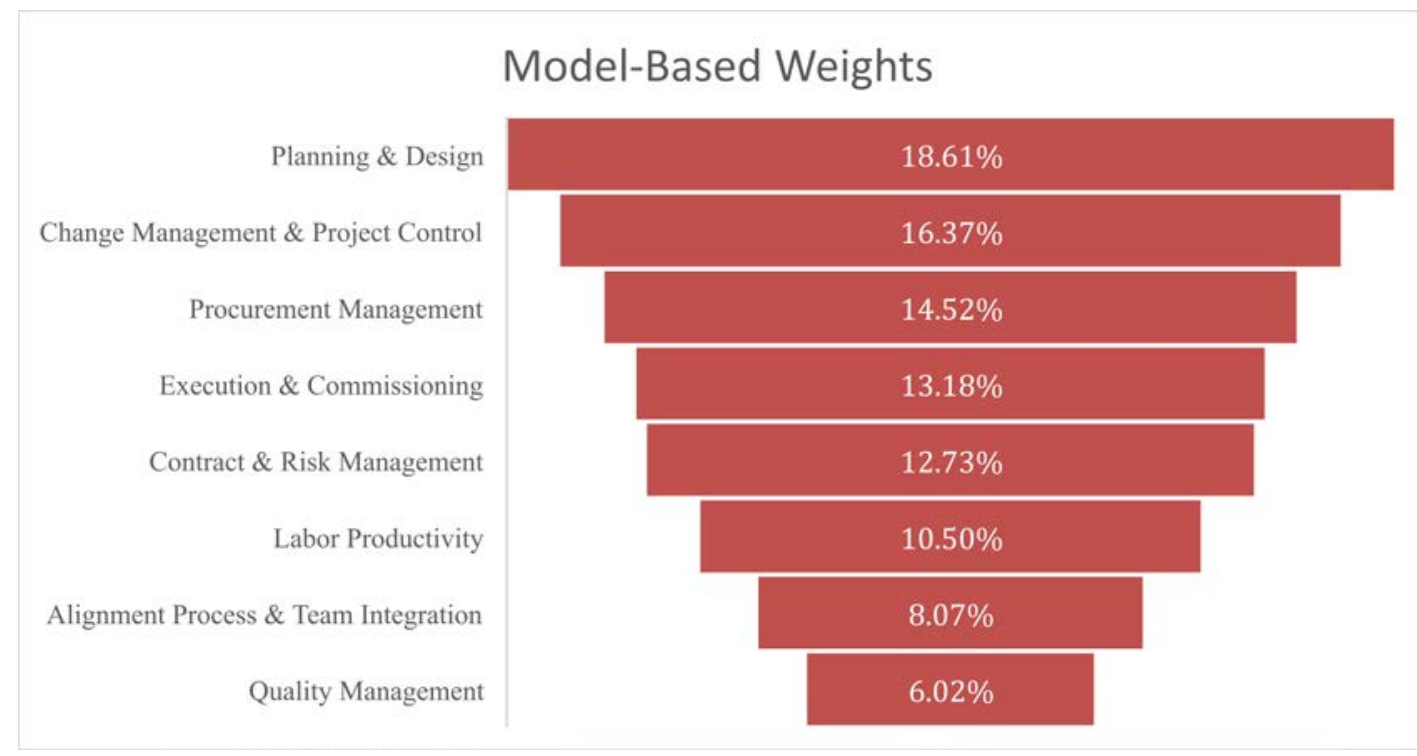

Figure 83: Weights breakdown of the CCA categories

To simplify the computations for the users, the factors are reverted from their standardized values to their original values using the mean and standard deviation as shown in the following equations:

$$
\mathrm{CCA}=\sum_{i=1}^{n} w_{i} z_{i}=\sum_{i=1}^{n} w_{i}\left(\frac{x_{i j}-\bar{x}_{j}}{ \pm s_{j}}\right)=\sum_{i=1}^{n}\left(\frac{w_{i}}{ \pm s_{j}}\right) x_{i j}-\sum_{i=1}^{n}\left(\frac{w_{i} \bar{x}_{j}}{ \pm s_{j}}\right)=\beta+\sum_{j=1}^{n} \alpha_{j} x_{i j}
$$

Where

$$
\alpha_{j}=\frac{w_{i}}{ \pm s_{j}} \text { and } \beta=\frac{w_{i} \bar{x}_{j}}{ \pm s_{j}}
$$

A simplified equation is developed to accommodate the unstandardized data. Therefore, the aggregation of this transformation for the eight-competency categories resulted in the constant term in the following equation: 
CCA Standardized Score $=$

$$
\begin{gathered}
-2.83+0.360 \mathrm{AL} \& \mathrm{~T}+0.754 \mathrm{PL} \& \mathrm{D}+0.528 \mathrm{E} \& \mathrm{C}+0.574 \text { Proc }+0.223 \mathrm{QM} \\
+0.659 \mathrm{CMPC}+0.394 \mathrm{RM}+0.352 \mathrm{LP}
\end{gathered}
$$

The results of the CCA score are in a standardized form, i.e. a value that can be positive or negative. For better visualization and comparison of projects to one another, the authors have decided to scale the generated CCA score to have a range between $5 \%$ to $95 \%$. A range of [5, 95\%] was chosen instead of $[0,100 \%]$, as it is not reasonable to presume the worst possible project competency to be $0 \%$ and the best possible project competency to be $100 \%$. Therefore, the equation below eliminates errors that stem from standardization, while it also provides a userfriendly methodology to compute the final CCA score of a range between 5\% to 95\%:

$$
C C A_{\text {final }}=\frac{C C A_{i}-C C A_{\min }}{C C A_{\max }-C C A_{\min }}(0.95-0.05)+0.05
$$

where

$$
C C A_{\min }=\min \left\{C C A_{i} \mid 1 \leq i \leq m\right\} \quad \text { and } \quad C C A_{\max }=\max \left\{C C A_{i} \mid 1 \leq i \leq m\right\}
$$

The final CCA score equation can be written in this form:

$$
\begin{aligned}
C C A_{\text {final }} & =0.05+\left(\frac{C C A_{i}+2.137}{2.954}\right) \times 0.9 \\
& =0.701+0.305 \times C C A_{i}
\end{aligned}
$$

Using these computed weights, the final scaled CCA score for any project can be computed using the equation below, given that all metrics are unstandardized. The following equation scales the generated CCA score to have a range between 5\% to 95\%. 
CCA Score $=-0.162+0.109$ AL\&T +0.230 PL\&D $+0.161 \mathrm{E} \& \mathrm{C}+0.175$ Proc +

$0.068 \mathrm{QM}+0.200 \mathrm{CMPC}+0.120 \mathrm{RM}+0.107 \mathrm{LP}$

\subsection{Competency Category Assessment (CCA) score vs Project Status}

This section presents the relationship between the CCA score and the project status. A QQ plot and Shapiro-Wilk test are conducted to assess the normality assumption. The following Q-Q plot shows a clear departure from normality at the overall level of the dataset.
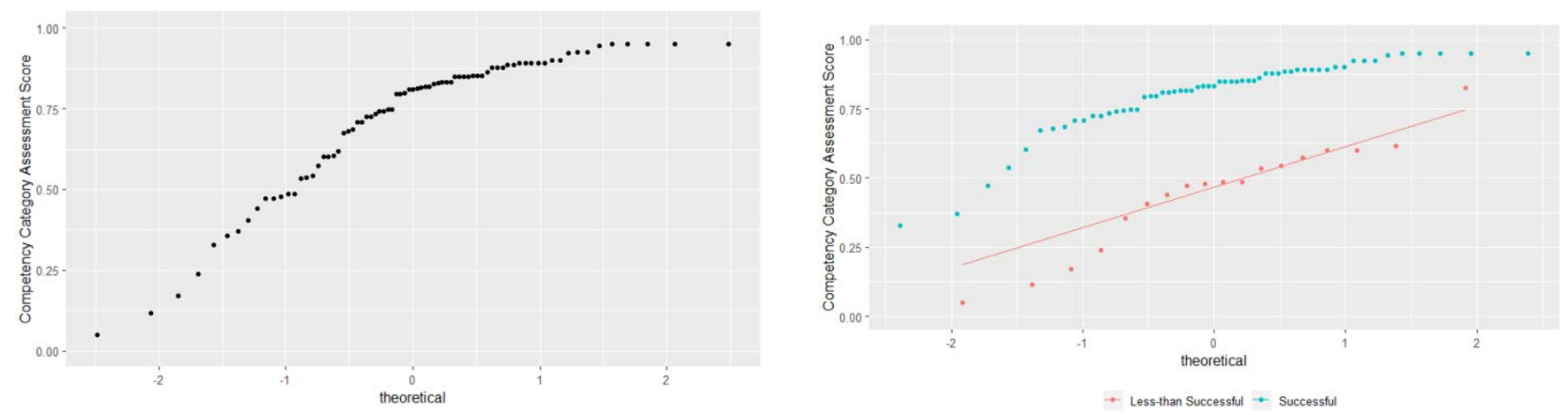

Figure 84: Q-Q plot of the CCA score

As with the other analysis, the normality assumption is not valid, as it was confirmed at a $95 \%$ confidence level by the Shapiro-Wilk test, which is shown in the table below.

Table 58: Shapiro-Wilk Test for Competency Category Assessment Score

Parameter

Overall

Successful Projects

Less-than-successful Projects $p$-value

0.000

0.000

0.312
Significance at 95\% Confidence Level

Significant

Significant

Not Significant 
The differences between successful and less-than-successful projects are shown by using box-and-whisker plots. It is visually evident that successful and less-than-successful projects have similar variability to each other, as it was illustrated from the level of compaction of the boxplot and that was evident from the Fligner-Killeen test returning a p-value of 0.113 , confirming the equal variance of both project statuses at a 95\% confidence level. The Wilcoxon rank-sum test returned a p-value of 0.000 , which demonstrates that the data provides evidence to reject the null hypothesis at a 5\% significance level, thus concluding at a 95\% confidence level that there is a statistically significant evidence that CCA score varies across the status of the project. Thus, it shows that projects that have a higher competency level are more likely to have good performance, while projects that have a poor competency level are more likely to have poor performance.

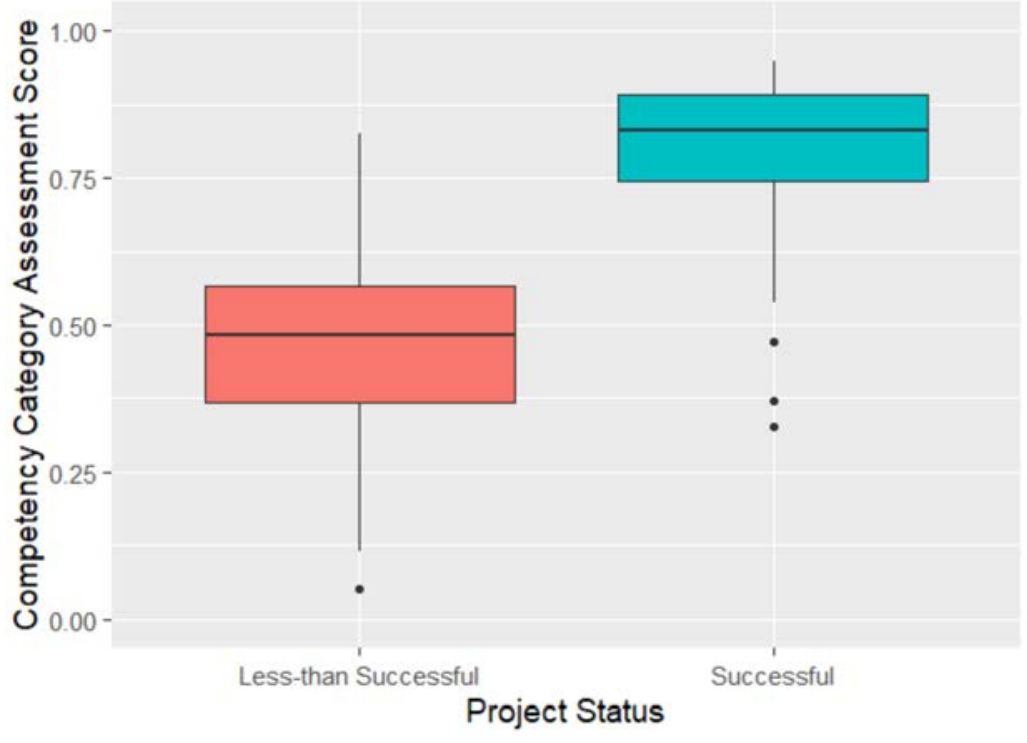

Figure 85: Boxplot of CCA score

The table of descriptive statistics below shows that the median/mean for CCA score for successful projects (83.2/80.4\%) is higher than less-than-successful projects (48.1/44.4\%), which shows the importance for having a high competency level in all previously mentioned categories across the project's lifecycle for project success. 
Table 59: Descriptive Statistics for Competency Category Assessment Score

\begin{tabular}{ccc}
\hline \hline Statistical Test & Successful Projects & Less-than-successful Projects \\
\hline \hline Mean & $80.4 \%$ & $44.4 \%$ \\
Maximum & $95.0 \%$ & $82.6 \%$ \\
$75 \%$ Quantile & $89.1 \%$ & $56.6 \%$ \\
$50 \%$ Quantile & $83.2 \%$ & $48.1 \%$ \\
25\% Quantile & $74.5 \%$ & $36.8 \%$ \\
Minimum & $32.7 \%$ & $5.0 \%$ \\
\hline \hline
\end{tabular}

\subsection{Regression Analysis}

Regression analysis is performed to examine the degree of association between the CCA score and the PPA score, as shown in Figure 85. The main objective is to determine the correlation between the competency categories and the PPA score as a method of checking the reliability of the overall success of the second model, since the goal is to maximize the correlation between each competency category score and the PPA score. The regression analysis is also used to predict the PPA score from the final computed CCA score. The assumptions of regression analysis are checked as shown in Figure 86. The assumption of constant variance seems to be reasonably plausible based on the residual plot. Similarly, the assumption of constant effects is met since the errors are normally distributed, as illustrated from the Q-Q plot. It is important to check that there are no outlying $\mathrm{X}$ observations. This check is done by calculating the leverage values and making sure that no observation has a leverage value greater than twice the mean leverage value. The last test is calculating the Cook's distance, which is an aggregate measure that accounts for the 
influence of deleting one observation on the fitted values of all 57 observations at a time. The percentile value of Cook's distance should be calculated from the F distribution and for each given observation the percentile value should be less than 20 , thus this observation has little influence on all 57 fitted values. For this model, the percentile values of all Cook's distances in the F distribution were below $20 \%$, indicating that none of the points in the model is influential enough to be excluded from the model. Given that the previous assumptions of regression analysis are met, the derived model has a coefficient of multiple determination $\left(R^{2}\right)$ of $51.1 \%$, a reasonable value for the type of data analyzed, as shown in Figure 5. The correlation accuracy for this relationship is $71.5 \%$. Such a strong correlation verifies the reliability and reasonability of the developed model.

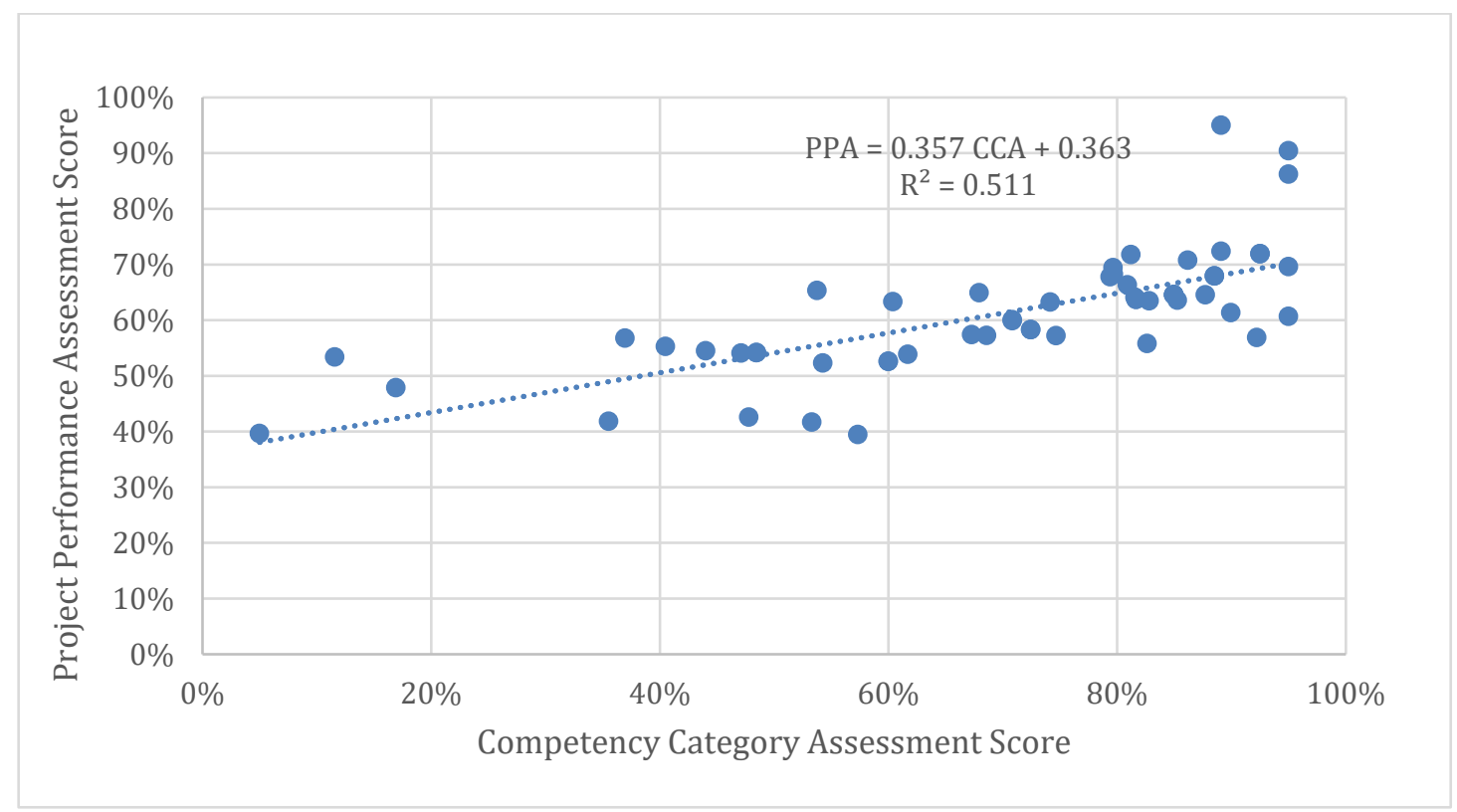

Figure 86: Linear regression model CCA vs PPA 

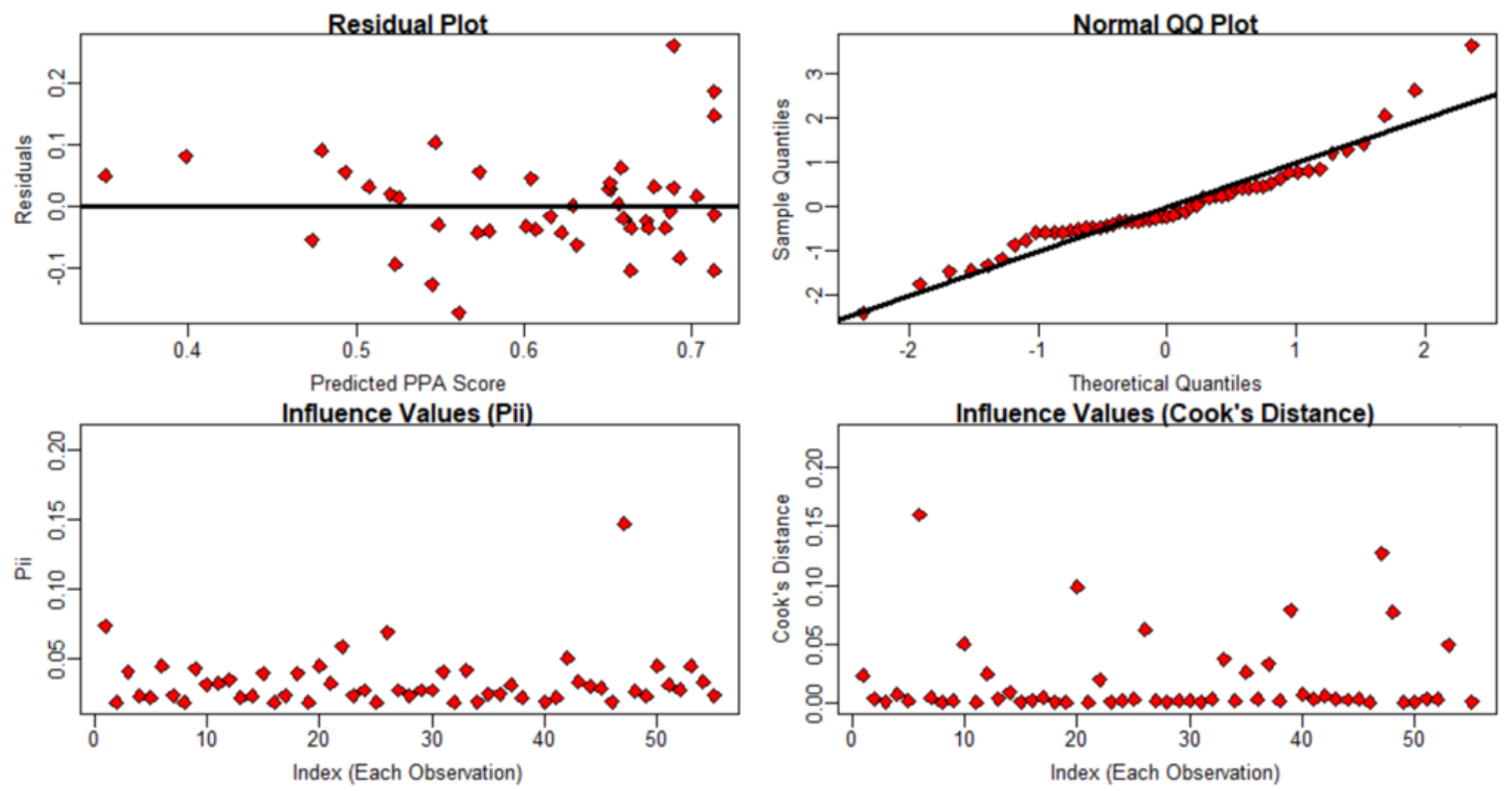

Figure 87: Checking the regression assumption

\subsection{CCA Model Validation}

One way to detect overfitting and determine the prediction accuracy of proposed models is to use five-fold cross validation. In cross validation, the training model is generated randomly using $80 \%$ of the data, which is used to predict the remaining $20 \%$ of the data (test dataset). This process is repeated five times and the prediction errors can be assessed using the MAPE and the deviation range (Hanna and Iskandar 2017). The model has a MAPE of 9.16\%, which is acceptable for the type of data analyzed. The deviation in the PPA score for each project is computed. All 57 projects are predicted within $\pm 44 \%$ from the actual PPA score; 25 projects are predicted within $\pm 5 \%, 20$ projects are predicted within $\pm 15 \%, 12$ projects are predicted within $\pm 30 \%$, one project is predicted within $\pm 36 \%$, and one project is predicted within $\pm 44 \%$. The results are presented in Table 60 . 
Table 60: Second Mathematical Model Validation

\begin{tabular}{|c|c|c|c|c|c|c|c|c|c|c|c|c|c|c|}
\hline Projects & AL\&T & PL\&D & $E \& C$ & Proc & $\mathbf{Q M}$ & СMPC & CNRM & $\mathbf{L P}$ & CCA & $\begin{array}{c}\text { Adjusted } \\
\text { CCA }\end{array}$ & $\begin{array}{c}\text { Predicted } \\
\text { PPA }\end{array}$ & $\begin{array}{c}\text { Actual } \\
\text { PPA }\end{array}$ & $\begin{array}{c}\% \\
\text { Deviation }\end{array}$ & $\begin{array}{l}\text { \% Absolute } \\
\text { Deviation }\end{array}$ \\
\hline 1 & 0.05 & 0.23 & 0.05 & 0.66 & 0.95 & 0.27 & 0.95 & 0.95 & -1.13 & 0.36 & 0.49 & 0.42 & $-17.08 \%$ & $17.08 \%$ \\
\hline 2 & 0.63 & 0.50 & 0.80 & 0.66 & 0.95 & 0.66 & 0.95 & 0.95 & -0.07 & 0.68 & 0.61 & 0.65 & $6.71 \%$ & $6.71 \%$ \\
\hline 3 & 0.89 & 0.67 & 0.43 & 0.76 & 0.25 & 0.35 & 0.05 & 0.95 & -0.71 & 0.48 & 0.54 & 0.54 & $1.01 \%$ & $1.01 \%$ \\
\hline 4 & 0.75 & 0.49 & 0.68 & 0.47 & 0.95 & 0.76 & 0.50 & 0.95 & -0.32 & 0.60 & 0.58 & 0.63 & $8.61 \%$ & $8.61 \%$ \\
\hline 5 & 0.77 & 0.73 & 0.95 & 0.66 & 0.95 & 0.79 & 0.95 & 0.95 & 0.32 & 0.80 & 0.65 & 0.68 & $5.13 \%$ & $5.13 \%$ \\
\hline 6 & 0.95 & 0.95 & 0.95 & 0.95 & 0.95 & 0.95 & 0.95 & 0.95 & 0.82 & 0.95 & 0.70 & 0.90 & $22.36 \%$ & $22.36 \%$ \\
\hline 7 & 0.32 & 0.23 & 0.95 & 0.95 & 0.95 & 0.37 & 0.95 & 0.95 & -0.33 & 0.60 & 0.58 & 0.53 & $-9.76 \%$ & $9.76 \%$ \\
\hline 8 & 0.95 & 0.61 & 0.66 & 0.76 & 0.95 & 0.78 & 0.54 & 0.95 & 0.02 & 0.71 & 0.62 & 0.60 & $-2.51 \%$ & $2.51 \%$ \\
\hline 9 & 0.46 & 0.47 & 0.51 & 0.47 & 0.30 & 0.37 & 0.95 & 0.95 & -0.75 & 0.47 & 0.53 & 0.54 & $1.77 \%$ & $1.77 \%$ \\
\hline 10 & 0.77 & 0.65 & 0.95 & 0.53 & 0.53 & 0.31 & 0.95 & 0.05 & -0.55 & 0.53 & 0.55 & 0.42 & $-32.77 \%$ & $32.77 \%$ \\
\hline 11 & 0.89 & 0.95 & 0.95 & 0.95 & 0.95 & 0.66 & 0.95 & 0.95 & 0.61 & 0.89 & 0.68 & 0.68 & $0.06 \%$ & $0.06 \%$ \\
\hline 12 & 0.95 & 0.95 & 0.95 & 0.66 & 0.95 & 0.95 & 0.95 & 0.95 & 0.65 & 0.90 & 0.68 & 0.61 & $-11.54 \%$ & $11.54 \%$ \\
\hline 13 & 0.66 & 0.52 & 0.32 & 0.66 & 0.95 & 0.69 & 0.95 & 0.95 & -0.28 & 0.62 & 0.58 & 0.54 & $-8.31 \%$ & $8.31 \%$ \\
\hline 14 & 0.95 & 0.73 & 0.95 & 0.66 & 0.95 & 0.77 & 0.95 & 0.95 & 0.37 & 0.81 & 0.65 & 0.72 & $9.03 \%$ & $9.03 \%$ \\
\hline 15 & 0.95 & 0.84 & 0.95 & 0.95 & 0.95 & 0.95 & 0.95 & 0.95 & 0.74 & 0.93 & 0.69 & 0.72 & $3.62 \%$ & $3.62 \%$ \\
\hline 16 & 0.60 & 0.48 & 0.95 & 0.66 & 0.73 & 0.69 & 0.95 & 0.95 & -0.05 & 0.69 & 0.61 & 0.57 & $-6.18 \%$ & $6.18 \%$ \\
\hline 17 & 0.32 & 0.23 & 0.95 & 0.95 & 0.95 & 0.37 & 0.95 & 0.95 & -0.33 & 0.60 & 0.58 & 0.53 & $-9.76 \%$ & $9.76 \%$ \\
\hline 18 & 0.95 & 0.84 & 0.95 & 0.95 & 0.95 & 0.95 & 0.95 & 0.95 & 0.74 & 0.93 & 0.69 & 0.72 & $3.62 \%$ & $3.62 \%$ \\
\hline 19 & 0.95 & 0.61 & 0.66 & 0.76 & 0.95 & 0.78 & 0.54 & 0.95 & 0.02 & 0.71 & 0.62 & 0.60 & $-2.84 \%$ & $2.84 \%$ \\
\hline 20 & 0.95 & 0.95 & 0.95 & 0.95 & 0.95 & 0.95 & 0.95 & 0.95 & 0.82 & 0.95 & 0.70 & 0.86 & $18.55 \%$ & $18.55 \%$ \\
\hline 21 & 0.89 & 0.95 & 0.95 & 0.95 & 0.95 & 0.66 & 0.95 & 0.95 & 0.61 & 0.89 & 0.68 & 0.68 & $0.06 \%$ & $0.06 \%$ \\
\hline 22 & 0.26 & 0.13 & 0.34 & 0.47 & 0.05 & 0.05 & 0.54 & 0.05 & -1.92 & 0.12 & 0.40 & 0.53 & $24.27 \%$ & $24.27 \%$ \\
\hline 23 & 0.57 & 0.28 & 0.56 & 0.24 & 0.70 & 0.95 & 0.54 & 0.05 & -0.97 & 0.41 & 0.51 & 0.55 & $8.17 \%$ & $8.17 \%$ \\
\hline 24 & 0.60 & 0.76 & 0.84 & 0.95 & 0.73 & 0.86 & 0.95 & 0.95 & 0.38 & 0.82 & 0.65 & 0.64 & $-2.72 \%$ & $2.72 \%$ \\
\hline 25 & 0.75 & 0.69 & 0.84 & 0.95 & 0.95 & 0.95 & 0.95 & 0.95 & 0.49 & 0.85 & 0.67 & 0.65 & $-3.22 \%$ & $3.22 \%$ \\
\hline 26 & 0.58 & 0.77 & 0.66 & 0.95 & 0.95 & 0.46 & 0.95 & 0.95 & 0.08 & 0.72 & 0.62 & 0.58 & $-6.64 \%$ & $6.64 \%$ \\
\hline 27 & 0.11 & 0.57 & 0.44 & 0.05 & 0.05 & 0.57 & 0.75 & 0.95 & -1.09 & 0.37 & 0.49 & 0.57 & $12.79 \%$ & $12.79 \%$ \\
\hline
\end{tabular}




\begin{tabular}{|c|c|c|c|c|c|c|c|c|c|c|c|c|c|c|}
\hline 28 & 0.75 & 0.69 & 0.84 & 0.95 & 0.95 & 0.95 & 0.95 & 0.95 & 0.49 & 0.85 & 0.67 & 0.65 & $-3.22 \%$ & $3.22 \%$ \\
\hline 29 & 0.60 & 0.76 & 0.84 & 0.95 & 0.73 & 0.86 & 0.95 & 0.95 & 0.38 & 0.82 & 0.65 & 0.64 & $-2.72 \%$ & $2.72 \%$ \\
\hline 30 & 0.75 & 0.69 & 0.84 & 0.95 & 0.95 & 0.95 & 0.95 & 0.95 & 0.49 & 0.85 & 0.67 & 0.65 & $-3.22 \%$ & $3.22 \%$ \\
\hline 31 & 0.75 & 0.69 & 0.84 & 0.95 & 0.95 & 0.95 & 0.95 & 0.95 & 0.49 & 0.85 & 0.67 & 0.65 & $-3.22 \%$ & $3.22 \%$ \\
\hline 32 & 0.89 & 0.67 & 0.43 & 0.76 & 0.25 & 0.35 & 0.05 & 0.95 & -0.71 & 0.48 & 0.54 & 0.54 & $1.01 \%$ & $1.01 \%$ \\
\hline 33 & 0.58 & 0.77 & 0.66 & 0.95 & 0.95 & 0.46 & 0.95 & 0.95 & 0.08 & 0.72 & 0.62 & 0.58 & $-6.64 \%$ & $6.64 \%$ \\
\hline 34 & 0.58 & 0.43 & 0.84 & 0.24 & 0.95 & 0.51 & 0.26 & 0.95 & -0.73 & 0.48 & 0.53 & 0.43 & $-25.33 \%$ & $25.33 \%$ \\
\hline 35 & 0.58 & 0.43 & 0.95 & 0.66 & 0.95 & 0.86 & 0.54 & 0.95 & -0.09 & 0.67 & 0.60 & 0.57 & $-5.10 \%$ & $5.10 \%$ \\
\hline 36 & 0.95 & 0.95 & 0.95 & 0.24 & 0.95 & 0.95 & 0.95 & 0.95 & 0.41 & 0.83 & 0.66 & 0.56 & $-17.94 \%$ & $17.94 \%$ \\
\hline 37 & 0.95 & 0.70 & 0.95 & 0.66 & 0.75 & 0.95 & 0.95 & 0.95 & 0.42 & 0.83 & 0.66 & 0.63 & $-3.80 \%$ & $3.80 \%$ \\
\hline 38 & 0.81 & 0.64 & 0.84 & 0.95 & 0.72 & 0.52 & 0.05 & 0.05 & -0.54 & 0.54 & 0.55 & 0.65 & $15.05 \%$ & $15.05 \%$ \\
\hline 39 & 0.95 & 0.79 & 0.95 & 0.95 & 0.72 & 0.85 & 0.26 & 0.95 & 0.31 & 0.79 & 0.65 & 0.68 & $4.62 \%$ & $4.62 \%$ \\
\hline 40 & 0.37 & 0.05 & 0.05 & 0.05 & 0.05 & 0.16 & 0.05 & 0.95 & -2.14 & 0.05 & 0.38 & 0.40 & $3.95 \%$ & $3.95 \%$ \\
\hline 41 & 0.63 & 0.84 & 0.95 & 0.95 & 0.28 & 0.76 & 0.46 & 0.95 & 0.15 & 0.75 & 0.63 & 0.57 & $-10.03 \%$ & $10.03 \%$ \\
\hline 42 & 0.89 & 0.65 & 0.73 & 0.95 & 0.95 & 0.86 & 0.75 & 0.95 & 0.31 & 0.80 & 0.65 & 0.69 & $6.75 \%$ & $6.75 \%$ \\
\hline 43 & 0.69 & 0.11 & 0.80 & 0.95 & 0.30 & 0.88 & 0.05 & 0.05 & -0.86 & 0.44 & 0.52 & 0.54 & $4.55 \%$ & $4.55 \%$ \\
\hline 44 & 0.83 & 0.90 & 0.95 & 0.95 & 0.95 & 0.78 & 0.95 & 0.95 & 0.62 & 0.89 & 0.68 & 0.72 & $5.87 \%$ & $5.87 \%$ \\
\hline 45 & 0.72 & 0.63 & 0.84 & 0.76 & 0.75 & 0.76 & 0.05 & 0.05 & -0.52 & 0.54 & 0.56 & 0.52 & $-6.48 \%$ & $6.48 \%$ \\
\hline 46 & 0.83 & 0.80 & 0.95 & 0.95 & 0.95 & 0.75 & 0.95 & 0.95 & 0.53 & 0.86 & 0.67 & 0.71 & $5.22 \%$ & $5.22 \%$ \\
\hline 47 & 0.95 & 0.65 & 0.80 & 0.95 & 0.75 & 0.44 & 0.95 & 0.95 & 0.13 & 0.74 & 0.63 & 0.63 & $0.75 \%$ & $0.75 \%$ \\
\hline 48 & 0.89 & 0.29 & 0.05 & 0.24 & 0.95 & 0.20 & 0.05 & 0.05 & -1.74 & 0.17 & 0.42 & 0.48 & $11.53 \%$ & $11.53 \%$ \\
\hline 49 & 0.77 & 0.26 & 0.51 & 0.66 & 0.95 & 0.57 & 0.95 & 0.95 & -0.42 & 0.57 & 0.57 & 0.39 & $-43.97 \%$ & $43.97 \%$ \\
\hline 50 & 0.77 & 0.95 & 0.44 & 0.95 & 0.95 & 0.76 & 0.95 & 0.95 & 0.35 & 0.81 & 0.65 & 0.66 & $1.72 \%$ & $1.72 \%$ \\
\hline 51 & 0.95 & 0.95 & 0.95 & 0.95 & 0.95 & 0.95 & 0.95 & 0.95 & 0.82 & 0.95 & 0.70 & 0.70 & $-0.84 \%$ & $0.84 \%$ \\
\hline 52 & 0.95 & 0.95 & 0.95 & 0.95 & 0.95 & 0.59 & 0.95 & 0.95 & 0.58 & 0.88 & 0.68 & 0.65 & $-4.74 \%$ & $4.74 \%$ \\
\hline 53 & 0.95 & 0.75 & 0.95 & 0.66 & 0.95 & 0.95 & 0.95 & 0.95 & 0.50 & 0.85 & 0.67 & 0.64 & $-4.98 \%$ & $4.98 \%$ \\
\hline 54 & 0.95 & 0.95 & 0.95 & 0.95 & 0.95 & 0.95 & 0.95 & 0.95 & 0.82 & 0.95 & 0.70 & 0.61 & $-15.75 \%$ & $15.75 \%$ \\
\hline 55 & 0.95 & 0.91 & 0.95 & 0.95 & 0.95 & 0.86 & 0.95 & 0.95 & 0.73 & 0.92 & 0.69 & 0.57 & $-21.75 \%$ & $21.75 \%$ \\
\hline 56 & 0.89 & 0.84 & 0.78 & 0.95 & 0.95 & 0.95 & 0.95 & 0.95 & 0.62 & 0.89 & 0.68 & 0.95 & $28.33 \%$ & $28.33 \%$ \\
\hline 57 & 0.89 & 0.73 & 0.78 & 0.66 & 0.95 & 0.95 & 0.95 & 0.95 & 0.38 & 0.82 & 0.65 & 0.64 & $-2.04 \%$ & $2.04 \%$ \\
\hline
\end{tabular}




\begin{tabular}{|c|c|c|c|c|}
\hline Projects & Predicted PPA & Predicted Status & Actual PPA Score & Actual Status \\
\hline 1 & 0.49 & Less-than Successful & 0.42 & Less-than Successful \\
\hline 2 & 0.61 & Successful & 0.65 & Successful \\
\hline 3 & 0.54 & Less-than Successful & 0.54 & Less-than Successful \\
\hline 4 & 0.58 & Successful & 0.63 & Successful \\
\hline 5 & 0.65 & Successful & 0.68 & Successful \\
\hline 6 & 0.70 & Successful & 0.90 & Successful \\
\hline 7 & 0.58 & Successful & 0.53 & Less-than Successful \\
\hline 8 & 0.62 & Successful & 0.60 & Successful \\
\hline 9 & 0.53 & Less-than Successful & 0.54 & Less-than Successful \\
\hline 10 & 0.55 & Successful & 0.42 & Less-than Successful \\
\hline 11 & 0.68 & Successful & 0.68 & Successful \\
\hline 12 & 0.68 & Successful & 0.61 & Successful \\
\hline 13 & 0.58 & Successful & 0.54 & Less-than Successful \\
\hline 14 & 0.65 & Successful & 0.72 & Successful \\
\hline 15 & 0.69 & Successful & 0.72 & Successful \\
\hline 16 & 0.61 & Successful & 0.57 & Successful \\
\hline 17 & 0.58 & Successful & 0.53 & Less-than Successful \\
\hline 18 & 0.69 & Successful & 0.72 & Successful \\
\hline 19 & 0.62 & Successful & 0.60 & Successful \\
\hline 20 & 0.70 & Successful & 0.86 & Successful \\
\hline 21 & 0.68 & Successful & 0.68 & Successful \\
\hline 22 & 0.40 & Less-than Successful & 0.53 & Less-than Successful \\
\hline 23 & 0.51 & Less-than Successful & 0.55 & Less-than Successful \\
\hline 24 & 0.65 & Successful & 0.64 & Successful \\
\hline 25 & 0.67 & Successful & 0.65 & Successful \\
\hline 26 & 0.62 & Successful & 0.58 & Successful \\
\hline 27 & 0.49 & Less-than Successful & 0.57 & Successful \\
\hline 28 & 0.67 & Successful & 0.65 & Successful \\
\hline 29 & 0.65 & Successful & 0.64 & Successful \\
\hline 30 & 0.67 & Successful & 0.65 & Successful \\
\hline
\end{tabular}




\begin{tabular}{|c|c|c|c|c|}
\hline 31 & 0.67 & Successful & 0.65 & Successful \\
\hline 32 & 0.54 & Less-than Successful & 0.54 & Less-than Successful \\
\hline 33 & 0.62 & Successful & 0.58 & Successful \\
\hline 34 & 0.53 & Less-than Successful & 0.43 & Less-than Successful \\
\hline 35 & 0.60 & Successful & 0.57 & Successful \\
\hline 36 & 0.66 & Successful & 0.56 & Less-than Successful \\
\hline 37 & 0.66 & Successful & 0.63 & Successful \\
\hline 38 & 0.55 & Successful & 0.65 & Successful \\
\hline 39 & 0.65 & Successful & 0.68 & Successful \\
\hline 40 & 0.38 & Less-than Successful & 0.40 & Less-than Successful \\
\hline 41 & 0.63 & Successful & 0.57 & Successful \\
\hline 42 & 0.65 & Successful & 0.69 & Successful \\
\hline 43 & 0.52 & Less-than Successful & 0.54 & Less-than Successful \\
\hline 44 & 0.68 & Successful & 0.72 & Successful \\
\hline 45 & 0.56 & Successful & 0.52 & Less-than Successful \\
\hline 46 & 0.67 & Successful & 0.71 & Successful \\
\hline 47 & 0.63 & Successful & 0.63 & Successful \\
\hline 48 & 0.42 & Less-than Successful & 0.48 & Less-than Successful \\
\hline 49 & 0.57 & Successful & 0.39 & Less-than Successful \\
\hline 50 & 0.65 & Successful & 0.66 & Successful \\
\hline 51 & 0.70 & Successful & 0.70 & Successful \\
\hline 52 & 0.68 & Successful & 0.65 & Successful \\
\hline 53 & 0.67 & Successful & 0.64 & Successful \\
\hline 54 & 0.70 & Successful & 0.61 & Successful \\
\hline 55 & 0.69 & Successful & 0.57 & Successful \\
\hline 56 & 0.68 & Successful & 0.95 & Successful \\
\hline 57 & 0.65 & Successful & 0.64 & Successful \\
\hline
\end{tabular}


A confusion matrix presented in Table 61 shows the actual vs predicted project statuses. This helps us to find the accuracy of the model and avoid overfitting. The model has an $86.0 \%$ accuracy as shown in the equation below, which is acceptable for the type of data analyzed.

Table 61: Confusion Matrix of Actual vs Predicted Project Statuses for Training Datasets

\begin{tabular}{|l|l|c|c|}
\multicolumn{2}{|c|}{ Confusion Matrix } & \multicolumn{2}{c|}{ Predicted } \\
\cline { 2 - 4 } Actual & Successful Projects & Less-than Successful Projects \\
\cline { 2 - 4 } & Less-than Successful Projects & 39 & 1 \\
\hline
\end{tabular}

$$
\begin{gathered}
\text { Model Accuracy }=\frac{\text { True Positive }+ \text { True Negatives }}{\text { True Positive }+ \text { True Negatives }+ \text { False Positive }+ \text { False Negatives }} \times 100 \% \\
=\frac{49}{57} \times 100 \%=86.0 \%
\end{gathered}
$$

As a final step to provide an adequate assessment of the future prediction accuracy of the developed models, 20 new projects are collected and tested. The model has a MAPE of 9.64\%, and all 20 projects are predicted within $\pm 30 \%$ from the actual PPA score, ten projects are predicted within $\pm 5 \%$, five projects are predicted within $\pm 15 \%$, and five projects are predicted within $\pm 30 \%$. The results seem adequate for the type of data analyzed. The results are presented in Table 62. A confusion matrix presented in Table 63 shows the actual vs predicted project statuses. This helps us to find the accuracy of the model and avoid overfitting. The model has $90.0 \%$ accuracy as shown in the equation below, which is acceptable for the type of data analyzed. 
Table 62: New projects introduced to second mathematical model

\begin{tabular}{|c|c|c|c|c|c|c|c|c|c|c|c|c|c|c|}
\hline Projects & AL\&T & PL\&D & E\&C & Proc & $\mathbf{Q M}$ & CMPC & CNRM & $\mathbf{L P}$ & CCA & $\begin{array}{c}\text { Adjusted } \\
\text { CCA }\end{array}$ & $\begin{array}{l}\text { Predicted } \\
\text { PPA }\end{array}$ & $\begin{array}{c}\text { Actual } \\
\text { PPA }\end{array}$ & \% Deviation & $\begin{array}{l}\text { \% Absolute } \\
\text { Deviation }\end{array}$ \\
\hline 1 & 0.89 & 0.84 & 0.78 & 0.95 & 0.95 & 0.95 & 0.95 & 0.95 & 0.62 & 0.89 & 0.68 & 0.65 & $-4.62 \%$ & $4.62 \%$ \\
\hline 2 & 0.95 & 0.95 & 0.95 & 0.95 & 0.95 & 0.95 & 0.95 & 0.95 & 0.82 & 0.95 & 0.70 & 0.62 & $-13.24 \%$ & $13.24 \%$ \\
\hline 3 & 0.95 & 0.95 & 0.95 & 0.66 & 0.95 & 0.95 & 0.95 & 0.95 & 0.65 & 0.90 & 0.68 & 0.57 & $-21.04 \%$ & $21.04 \%$ \\
\hline 4 & 0.89 & 0.95 & 0.95 & 0.95 & 0.95 & 0.95 & 0.95 & 0.95 & 0.80 & 0.94 & 0.70 & 0.67 & $-4.28 \%$ & $4.28 \%$ \\
\hline 5 & 0.95 & 0.68 & 0.95 & 0.76 & 0.95 & 0.95 & 0.75 & 0.05 & 0.11 & 0.73 & 0.62 & 0.64 & $2.74 \%$ & $2.74 \%$ \\
\hline 6 & 0.83 & 0.85 & 0.95 & 0.34 & 0.95 & 0.95 & 0.95 & 0.95 & 0.35 & 0.81 & 0.65 & 0.70 & $6.50 \%$ & $6.50 \%$ \\
\hline 7 & 0.58 & 0.72 & 0.95 & 0.95 & 0.95 & 0.86 & 0.95 & 0.05 & 0.14 & 0.74 & 0.63 & 0.62 & $-0.92 \%$ & $0.92 \%$ \\
\hline 8 & 0.48 & 0.23 & 0.05 & 0.47 & 0.95 & 0.50 & 0.29 & 0.05 & -1.52 & 0.24 & 0.45 & 0.56 & $19.58 \%$ & $19.58 \%$ \\
\hline 9 & 0.38 & 0.22 & 0.20 & 0.66 & 0.30 & 0.54 & 0.95 & 0.05 & -1.23 & 0.33 & 0.48 & 0.62 & $22.17 \%$ & $22.17 \%$ \\
\hline 10 & 0.32 & 0.52 & 0.66 & 0.24 & 0.95 & 0.37 & 0.74 & 0.95 & -0.75 & 0.47 & 0.53 & 0.66 & $19.81 \%$ & $19.81 \%$ \\
\hline 11 & 0.78 & 0.47 & 0.95 & 0.66 & 0.95 & 0.83 & 0.95 & 0.95 & 0.15 & 0.75 & 0.63 & 0.64 & $0.90 \%$ & $0.90 \%$ \\
\hline 12 & 0.75 & 0.53 & 0.95 & 0.95 & 0.95 & 0.95 & 0.95 & 0.95 & 0.43 & 0.83 & 0.66 & 0.61 & $-8.45 \%$ & $8.45 \%$ \\
\hline 13 & 0.95 & 0.95 & 0.95 & 0.95 & 0.95 & 0.59 & 0.95 & 0.95 & 0.58 & 0.88 & 0.68 & 0.65 & $-4.74 \%$ & $4.74 \%$ \\
\hline 14 & 0.95 & 0.75 & 0.95 & 0.66 & 0.95 & 0.95 & 0.95 & 0.95 & 0.50 & 0.85 & 0.67 & 0.64 & $-4.98 \%$ & $4.98 \%$ \\
\hline 15 & 0.75 & 0.53 & 0.95 & 0.95 & 0.95 & 0.95 & 0.95 & 0.95 & 0.43 & 0.83 & 0.66 & 0.61 & $-8.45 \%$ & $8.45 \%$ \\
\hline 17 & 0.89 & 0.84 & 0.78 & 0.95 & 0.95 & 0.95 & 0.95 & 0.95 & 0.62 & 0.89 & 0.68 & 0.65 & $-4.62 \%$ & $4.62 \%$ \\
\hline 18 & 0.95 & 0.75 & 0.95 & 0.66 & 0.95 & 0.95 & 0.95 & 0.95 & 0.50 & 0.85 & 0.67 & 0.64 & $-4.90 \%$ & $4.90 \%$ \\
\hline 19 & 0.75 & 0.53 & 0.95 & 0.95 & 0.95 & 0.95 & 0.95 & 0.95 & 0.43 & 0.83 & 0.66 & 0.61 & $-8.45 \%$ & $8.45 \%$ \\
\hline 20 & 0.95 & 0.95 & 0.95 & 0.95 & 0.95 & 0.59 & 0.95 & 0.95 & 0.58 & 0.88 & 0.68 & 0.65 & $-4.74 \%$ & $4.74 \%$ \\
\hline
\end{tabular}




\begin{tabular}{|c|c|c|c|c|}
\hline Project & Predicted PPA & Predicted Status & Actual PPA & Actual Status \\
\hline $\mathbf{1}$ & 0.68 & Successful & 0.65 & Successful \\
\hline $\mathbf{2}$ & 0.70 & Successful & 0.62 & Successful \\
\hline $\mathbf{3}$ & 0.68 & Successful & 0.67 & Successful \\
\hline $\mathbf{4}$ & 0.70 & Successful & 0.64 & Successful \\
\hline $\mathbf{5}$ & 0.62 & Successful & Successful \\
\hline $\mathbf{6}$ & 0.65 & Successful & 0.70 & Successful \\
\hline $\mathbf{7}$ & 0.63 & Successful & 0.56 & Successful \\
\hline $\mathbf{8}$ & 0.45 & Less-than Successful & 0.62 & Successful \\
\hline $\mathbf{9}$ & 0.48 & Less-than Successful & 0.66 & Successful \\
\hline $\mathbf{1 1}$ & 0.53 & Less-than Successful & 0.64 & Successful \\
\hline $\mathbf{1 2}$ & 0.63 & Successful & 0.61 & Successful \\
\hline $\mathbf{1 3}$ & 0.66 & Successful & 0.65 & Successful \\
\hline $\mathbf{1 4}$ & 0.68 & Successful & 0.64 & Successful \\
\hline $\mathbf{1 6}$ & 0.67 & Successful & 0.61 & Successful \\
\hline $\mathbf{1 7}$ & 0.66 & Successful & 0.95 & Successful \\
\hline $\mathbf{1 8}$ & 0.68 & Successful & 0.65 & Successful \\
\hline $\mathbf{1 9}$ & 0.68 & Successful & 0.64 & Successful \\
\hline $\mathbf{2 0}$ & 0.67 & Successful & 0.61 & Successful \\
\hline
\end{tabular}


Table 63: Confusion Matrix of Actual VS Predicted Project Statuses for testing datasets

\begin{tabular}{|l|l|c|c|}
\multicolumn{2}{|c|}{ Confusion Matrix } & \multicolumn{2}{c|}{ Predicted } \\
\cline { 2 - 4 } Actual & Successful Projects & Less-than Successful Projects \\
\cline { 2 - 4 } & Less-than Successful Projects & 17 & 2 \\
\hline
\end{tabular}

$$
\begin{gathered}
\text { Model Accuracy }=\frac{\text { True Positive }+ \text { True Negatives }}{\text { True Positive }+ \text { True Negatives }+ \text { False Positive }+ \text { False Negatives }} \times 100 \% \\
=\frac{18}{20} \times 100 \%=90.0 \%
\end{gathered}
$$

\subsection{Summary and Conclusions}

This chapter highlighted the main objective of this dissertation, investigating the relationship between construction projects' competencies and performance. This was done by quantitatively assessing the relative contribution of the different competency categories to the PPA score (Chapter 4) and providing one unique comprehensive score named the CCA score. The results from the mathematical model showed that the top two scores in competency categories are: planning \& design and change management \& project control, having weights of $18.61 \%$ and $16.37 \%$ respectively. Contrastingly, the alignment process \& team integration and quality management competency categories scores have the lowest contribution in calculating the PPA score with weights of $8.07 \%$ and $6.02 \%$, respectively. It was also shown that the median/mean for CCA score for successful projects (83.2/80.4\%) is higher than less-than-successful projects (48.1/44.4\%). Last but not least, regression analysis was performed to examine the degree of association between the CCA and PPA score, given that the assumptions of regression analysis are met, the derived model has a coefficient of multiple determination $\left(R^{2}\right)$ of $51.1 \%$ and correlation accuracy of 71.5\%. Model validation was conducted using five-fold cross validation and testing of 20 new projects that were introduced to the model to check the reliability and reasonability of the developed model. 


\section{Chapter 7 Research Conclusions and Recommendations}

This chapter provides a review of the research conducted in this thesis and summarizes its contributions. Limitations of the research and recommendations for future research are also presented.

\subsection{Integrated Framework Applications}

This section offers a roadmap for practitioners and researchers to be able to predict the overall performance score of a project based on the investigation of the different project competencies as shown in Figure 87.

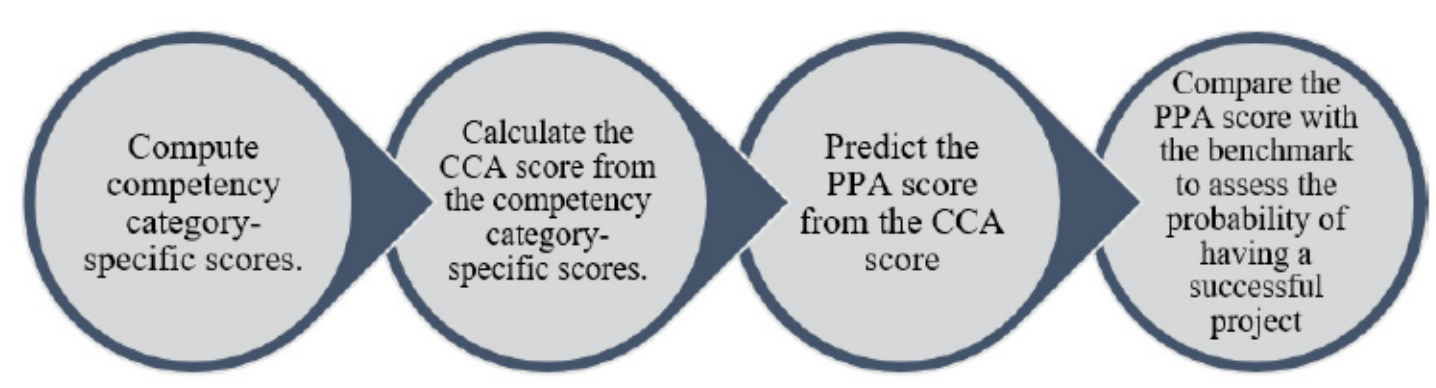

Figure 88: Roadmap for practitioners and researchers

\subsection{Summary of Results and Contributions}

Despite years of discussion, few comprehensive studies provide actionable leading competency factors to ease practitioners' adoption and implementation of these factors early in projects. Limitations in previous studies can be categorized into five principal limitations: 1) few studies did a comprehensive assessment and evaluation of the post-lagging performance in different performance areas; 2) none of the studies captured the different categories of project competencies that occur among and across project phases; 3) none of the studies did a 
comprehensive assessment and evaluation of the various competencies in different competency categories; 4) previous studies relied on subjective nature of the derived weights of the performance/competency metrics, as they were based on the experts' opinion; 5) prior research only gives a limited investigation of the relationship between project competencies as leading indicators of project performance improvement and quantifying the relative impact of each project competency on project performance.

This dissertation considered the performance of a total of 120 projects. The respondents were either general contractors/construction managers (GC/CM) (46\%), owners (28\%), subcontractors (24\%), or engineers (2\%). Most projects in the dataset were industrial (70.1\%), with buildings, infrastructure, and renovation representing $17.1 \%, 10.3 \%$, and $2.6 \%$, respectively. Each of the major project delivery systems (PDSs) were represented in the dataset. The most represented PDS was design-bid-build (DBB), with $27.5 \%$ of projects. Design-build (DB) (25\%) was the next most prevalent. Integrated project delivery (IPD), construction management-agent (CM-agent) and construction management at-risk (CMAR) were also represented with 18.3\%, 15.8\%, and 7.5\%, respectively. Other types of delivery systems such as Engineering, Procurement, and Construction (EPC), Engineering, Procurement, Construction, and Management (EPCM) and Engineering, Procurement, Construction, and Fabrication (EPCF) constituted 5.8\% of the projects.

This research has expanded the investigation of project competencies as leading indicators of project performance that occur among and across different project phases. The objectives of this research are met by using two robust, accurate, and data-driven weighting mathematical frameworks. The first mathematical model provides a systematic and structured process to evaluate project performance from the contractor's perspective with respect to a clear set of goals and objectives. As such, it creates a comprehensive performance score named the PPA score by 
combining the previously mentioned eight key performance metrics spanning over six performance areas: cost, schedule, productivity, safety, quality, and communication. Then, Kmeans clustering is used to benchmark successful projects from less-than-successful projects. The first mathematical model can also be used by practitioners and researchers to compute a customized competency category-specific score from various competency factors spanning nine categories: alignment \& team integration, planning \& design, procurement management, risk management, health, safety \& environmental, quality management, labor productivity, execution \& commissioning, and change management \& project control. Then, the second mathematical model is used to quantitatively assess the relative contribution of the different competency categories to the PPA score and provide one unique comprehensive score named the CCA score. Finally, a regression model is developed to examine the degree of association between the CCA score and PPA score, which construction practitioners can use to predict the overall performance score of a project.

The advantages of this research can be summarized as followed: 1) the developed mathematical frameworks reduce the subjectivity/uncertainty of the derived relative impacts (weights) of the project competency factors and performance metrics, as they are derived based on actual project data rather than experts' judgment; 2) construction practitioners can evaluate the competency and performance of projects in a formal and systematic way; where the CCA score can be used as an early warning sign to track a project's performance. Thus, the higher the CCA score, the more likely the project will have high an overall performance score and the lower the CCA score indicates that the project is least likely to be successful; 3) a benchmark of the PPA score was defined in this research to assess the overall project success. 
The first mathematical model is used to compute the PPA score for the different projects by combining eight key performance metrics spanning over six performance areas. The results showed that the top three metrics are: construction schedule growth, construction cost growth, and \% delta having $18.43 \%, 17.38 \%$, and $15.22 \%$, respectively. On the other hand, the percentage of rework and the number of lost-time injuries per 1 million dollars have the lowest contribution in calculating the PPA score. K-means clustering was then performed to benchmark successful projects from less-than-successful projects. The cluster plot displays the distinct classification of projects with limited overlap between the clusters. It was found that successful projects (cluster 1) have PPA scores with a range of 56\% - 95\%, while less-than-successful projects (cluster 2) have PPA scores with a range of 5\% - 55\%. Further analysis was conducted to verify that the selected metrics provide substantial contributions to the PPA model by checking the correlation coefficient. Model robustness is performed for validating the PPA model to check that the results are not highly determined by changes in the dataset. It was found that the PPA model has a MAPE of 7.0\% and the maximum and minimum total deviation of the eight-performance metrics weight were found to be $3.86 \%$ and $0.85 \%$ respectively. The analysis concluded that successful projects outperform less-than-successful projects, as they have $16 \%$ average cost savings, $42 \%$ average schedule reduction, $10 \%$ average productivity improvement, $7 \%$ average less rework, and $66 \%$ average increase in communication efficiency.

After computing the PPA score for the different projects, two statistical analysis steps were performed; the first step is a pairwise correlation, which is used to eliminate the problem of multicollinearity and the second step is to investigate the relationship between each of the competency factors as a stand-alone factor and the PPA score to determine the top competency factors that impact the PPA score. The number of the competency factors reduced from 217 to 46 
factors that span into eight categories instead of nine categories. It is worth mentioning that none of the health, safety, \& environmental factors were found to be correlated with the PPA score. The first mathematical model is also used to quantify the relative weights of each competency factors to each category. The top-weighted competency factors in each category, were found to be 1) AL\&T 20 : Project team has the expertise to take on the project; 2) PL\&D 31: IFC drawings are issued to support the construction activity; 3) Proc 23: All bulky materials needed for the start of construction are purchased \& onsite; 4) CNRM 16: Risks impacting the construction phase of the project are well assessed and CNRM 19: The risk monitoring and response framework are well defined in terms of cost; 5) QM 5: A quality control plan is communicated among the project team; 6) LP 2: A plan to secure and retain skilled labor and LP 6: a craft evaluation/qualification program are in place; 7) E\&C 10: A coordination plan is well defined to all project parties; 8) CMPC 12: Change management roles and responsibilities are well defined to project parties. After computing the competency-category specific scores for the eight categories, it was found that successful projects outperform less-than-successful projects by an average of $1.3 \times-1.9 \times$ in all the eight categories. This shows that being competent in all the categories will increase the likelihood of a project being successful.

In addition to these results, this thesis also fills a much-needed role in the literature by determining the relationship between the project competencies as leading indicators for project performance improvement. Recognizing this gap, this research developed the second mathematical model to compute the Competency Category Assessment (CCA) score. The CCA score incorporates a set of competency category-specific scores with varying degrees of impact (i.e. weights) on the PPA score. The results highlighted the importance of pre-construction planning \& design and change management \& project control towards project success, as they are the highest 
weighted categories, $18.61 \%$, and $16.37 \%$, respectively. Therefore, the construction industry should focus more on preconstruction activities because they are a critical link to all future operations of a project. In addition, having a change management plan and a successful progress monitoring plan throughout a project's life cycle are important methods of tackling performance deficiencies and productivity loss. On the other hand, the alignment process \& team integration and quality management competency categories scores have the lowest contribution in calculating the PPA score, with weights of $8.07 \%$ and $6.02 \%$, respectively. It was also shown that the median/mean for CCA score for successful projects (83.2/80.4\%) is higher than less-than-successful projects (48.1/44.4\%). Last, but not least, regression analysis is performed to examine the degree of association between the CCA score and PPA score; given that the assumptions of regression analysis are met, the derived model has a coefficient of multiple determination $\left(R^{2}\right)$ of $51.1 \%$ and correlation accuracy of $71.5 \%$. Model validation is conducted using five-fold cross validation and the testing of 20 new projects introduced to the model to check the reliability and reasonability of the developed model.

\subsection{Research Limitations and Future Recommendations}

One possible limitation of this study is that in all 120 studied projects, there were few injuries or fatalities incidents recorded. Therefore, these techniques should be used with caution in other projects that may have more injuries or fatalities recorded incidents, as the optimized weights may not be the same as what was proposed in this study.

The state-of-the-art techniques used in this research should be considered for further development in future work in order to maximize the practical benefits of the frameworks developed. In this study, each competency factor and performance metric in the model is 
independent of all the others. Therefore, future work may consider possible interactions between the different competency categories when computing the relative contribution of different competency categories scores to the PPA score, as well as the interactions between the different performance metrics in computing the final performance score. Another possible limitation of this study is to evaluate the same projects at varying stages of the projects' life cycle, which future research should consider validating the predicted project performance.

Finally, this research considered the project as the only unit of measurement. Future work should contemplate expanding project competencies and performance assessment from the project level to the organizational level. Once enough data relevant to the different contexts and the organizational level are collected, critical competencies at the organizational and project levels can be identified. Then, an investigation can be conducted to study the relationship between organizational and project level competencies and performance. 


\section{Appendix 1 - References}

Ahire, S. L., and Golhar, D. Y. (1996). Quality management in large vs. small firms. J. Small Bus. Manage., 34(2), 1-13.

Almasi, A. (2014). Pre-commissioning, commissioning and start-up of industrial plants and machineries.” Aust. J. Mech. Eng. 12 (2), 257-263. https://doi.org/10.7158/M12-101.2014.12.2

Alroomi, A., Jeong, D. H. S., and Oberlender, G. D. (2011). Analysis of cost-estimating competencies using criticality matrix and factor analysis. J. Constr. Eng. Manage., 138(11):1270-1280.

Arcadis. (2016). Global Construction Dispute Report. $<$ https://www.arcadis.com/media/3/E/7/\%7B3E7BDCDC-0434-4237-924F 739240965A90\%7DGlobal\%20Construction\%20Disputes\%20Report\%202016.pdf>

Battikha, M. G. (2008). Reasoning mechanism for construction nonconformance root-cause analysis. J. Constr. Eng. Manage., 10.1061/(ASCE)0733-9364(2008)134:4(280), 280-288.

Barber, P., Graves, G. A., Hall, M., Shearth, D., and Tomkins, C. (2000). The cost of quality failures in major civil engineering projects. Int. J. Qual. Reliab. Manage., 17(4/5), 479-492.

Barry, W., Leite, F., and O’Brien, W. J. (2015). The late deliverable risk catalog: Evaluating the impacts and risks of late deliverables to construction sites. Journal of Construction Engineering and Management, 141(4), 10.1061/(ASCE)CO.1943- 7862.0000950, 04014087.

Bingham, E., Gibson, G. E., and El Asmar, M. (2019). Identifying team selection and alignment factors by delivery method for transportation projects. Journal of Construction Engineering and Management, 145(10), 04019061

Briscoe, G., Dainty, A., Millett, S., and R. Neale (2004). Client-led strategies for construction supply chain improvement. Construction Management and Economics, 22 (2), 193-201.

Bubshait, A. A., and T. H. Al-Atiq. 1999. ISO 9000 quality standards in construction. J. Manage. Eng. 15 (6), 41-46. https://doi-org.ezproxy.library.wisc.edu/10.1061/(ASCE)0742-

597X(1999)15:6(41).

Bunni, N. G. (1985). The Spectrum of Risks in Construction. Rep. of the Standing Committee on Professional Liability, Fédération Internationale des Ingénieurs-Conseils, Lausanne, Switzerland.

Carpenter, N., and Bausman, C. D. (2016). Project delivery method performance for public school construction: Design-bid-build versus CM at risk. Journal of Construction Engineering \& Management, 142(10).

Cheeks, Richard J. (2003), “Multistep Dispute Resolution in Design and Construction Industry”. Journal of Professional Issues in Engineering Education and Practice, volume 129, pp 84-90. 
Cheng, Y. M., \& Leu, S. S. (2011). Integrating data mining with KJ method to classify bridge construction defects. Expert Systems with Applications, 38(6), 7143-7150.

Chen, J., and Hsu, S. C. (2007). "Hybrid ANN-CBR model for disputed change orders in construction projects.” Autom. Constr., 17(1), 56-64.

CII-RT-319 (Construction Industry Institute - Research Team 039) (2016). Transforming the industry: Making the case for AWP as a standard (best practices). CII, Austin, TX.

Davis, K., Ledbetter, W. B., and Burati, J. L. (1989). Measuring design and construction quality costs. J. Constr. Eng. Manage., 115(3), 385-400.

Dozzi, S. P., and AbouRizk, S. M. (1993). Productivity in Construction, Institute for Research in Construction, National Research Council, Ottawa, ON, Canada.

El Asmar, Mounir, Hanna, A., and Wei-Yin Loh (2013). Quantifying Performance for the Integrated Project Delivery System as Compared to Established Delivery Systems. Journal of Construction Engineering and Management, 139(11), 1-14, doi:10.1061/(ASCE)CO.19437862.0000744 .

Elzarka, H. M. (2009). Best Practices for Procuring Commissioning Services. Journal of Management in Engineering, 25(3), 155-164.

Eriksson, P.E., Nilsson, T. (2008). Partnering the construction of a Swedish pharmaceutical plant: case study. Journal of Management in Engineering, 24(4), 227-233.

Eriksson, P.E. (2008a). “Achieving suitable coopetition in buyer-supplier relationships: the case of AstraZeneca. Journal of Business to Business Marketing, 15(4), 425-454.

Eriksson, P.E. (2008b) Effects on coopetition in client-contractor relationships. Journal of Construction Engineering and Management, 134 (2) ,103-111.

Eriksson, Per Erik, and Mats Westerberg (2011). Effects of cooperative procurement procedures on construction project performance: A conceptual framework. International Journal of Project Management, 29(2), 197-208, doi:10.1016/j.ijproman.2010.01.003.

Ernzen, J., and C. Schexnayder. 2000. One company’s experience with design/build: Labor cost risk and profit potential. J. Constr. Eng. Manage. 126 (1): 10-14, https://doi.org/10.1061/(ASCE)0733-9364

Everitt, B. S., Landau, S., Leese, M., and Stahl, D. (2011). Cluster Analysis. Chichester, UK: John Wiley \& Sons, 5th edition. Cited on p. 165, 166, 171, 176, 180, 198.

Fallahnejad, M. H. (2013). Delay causes in Iran gas pipeline projects. Int. J. Project Manage., 31(1), 136-146. 
Fung, H. P., and Siow, H. L. (2013). Relationship between team satisfaction and project performance as perceived by project managers in Malaysia-A mixed methods study. Open J. Soc. Sci. Res., 1(9), 238-249.

García-Herrero, S., Mariscal, M. A., García-Rodríguez, J., and Ritzel, D. O. (2012). Working conditions, psychological/physical symptoms and occupational accidents. Bayesian network models." Safety Science, 50(9), 1760-1774.

Georgy, M. E. (2000). Utility-based Neurofuzzy Approach for Engineering Performance Assessment in Industrial Construction Projects. Ph.D. dissertation, School of Civil Engineering, Purdue Univ., West Lafayette, Ind.

Georgy, Maged E., Chang, Luh-Maan, and Zhang, Lei. Prediction of engineering performance: A neurofuzzy approach. Journal of Construction Engineering and Management, 131(5), 548-57, doi:10.1061/(ASCE)0733-9364(2005)131:5(548).

Goodrum, P. M., C. T. Haas, C. Caldas, D. Zhai, J. Yeiser, and D. Homm (2011). Model to predict the impact of a technology on construction productivity. Journal of Construction Engineering and Management, 137 (9): 678-688. https://doi.org /10.1061/(ASCE)CO.19437862.0000328 .

Gordon, A. D. (1987). A review of hierarchical classification. Journal of the Royal Statistical Society, Series A, 150, 119-137. Cited on p. 165.

--. (1999). Classification, Boca Raton, FL: Chapman \& Hall/CRC, 2nd edition. Cited on p. 165.

Griffith, A. F., and G. E. Gibson (2001). Alignment during pre-project planning. Journal of Construction Engineering and Management, 17 (2): 69-76. https://doi.org/10.1061/(ASCE) 0742-597X(2001)17:2(69).

Günhan, S., Arditi, D., and Doyle, J. (2007). Avoiding Change Orders in Public School Construction. Journal of Professional Issues in Engineering Education and Practice, 133(1), 6773.

Gurmu, A. T., and Aibinu, A. A. (2017). Construction equipment management practices for improving labor productivity in multistory building construction projects. Journal of Construction Engineering and Management, 143(10), 04017081.

Han, S., Saba, F., Lee, S., Mohamed, Y., and Pena-Mora, F. (2014). Toward an understanding of the impact of production pressure on safety performance in construction operations. Accident Analysis and Prevention, 68(7), 106-116.

Hanna, A. S. (2001). Quantifying the impact of change orders on electrical and mechanical labor productivity. Research Rep. No. 158-11. Austin, TX: Construction Industry Institute. 
Hanna, A. S. (2016). Benchmark performance metrics for integrated project delivery. Journal of Construction Engineering and Management, 142(9), 04016040.

Hanna, A. S. (2010). “Construction labor productivity management and methods improvement.” Hanna Consulting, Madison, Wisconsin. ISBN-13: 978-0-9829042-0-6.

Hanna, A. S., C. S. Taylor, and K. T. Sullivan. (2005). Impact of extended overtime on construction labor productivity. J. Constr. Eng. Manage. 131 (6): 734-739. https://doi.org/10.1061/(ASCE)0733-9364(2005)

Hanna, A. S., Camlic, R., Peterson, P. A., and Lee, M. J. (2004). Cumulative effect of project changes for electrical and mechanical construction. J. Constr. Eng. Manage., 130(6), 762-771.

Hanna, A. S., and Iskandar, Karim A. (2018).Factors Affecting Construction Labor Productivity: Qualitative and Quantitative Assessment. Construction Research Congress 2018, 597-607, doi: \{10.1061/9780784481271.058\}. https://ascelibrary.org/doi/abs/10.1061/9780784481271.058.

Hanna, A. S., and Skiffington, Mark A. (2010). Effect of preconstruction planning effort on sheet metal project performance. Journal of Construction Engineering and Management, 136(2), 23541, doi:10.1061/(ASCE)0733-9364(2010)136:2(235).

Hanna, A. S., and Swanson, J. (2007). Risk Allocation by Law-Cumulative Impact of Change Orders. Journal of Professional Issues in Engineering Education and Practice, 133(1), 60-66.

Hinze, J., Hallowell, M., and Baud, K. (2013). Construction-safety best practices and relationships to safety performance. Journal of Construction Engineering and Management, 139(10), 04013006.

Harmon, M. J. (2003), “Dispute Review Boards and Construction Conflicts: Attitudes and Opinions of Construction Industry Members”, PhD Dissertation, Nova Southeastern University, USA.

Hwang, B., Zhao, X., and Goh, K. J. (2014). Investigating the client related rework in building projects: The case of Singapore. Int. J. Project Manage., 32(4), 698-708.

Hwang, Seokyon, and Liang Y. Liu (2005). Proactive project control using productivity data and time series analysis. Proceedings of the 2005 ASCE International Conference on Computing in Civil Engineering, 217, 1925-35.

Ibrahim, M., Hanna, A., and Kievet, D. (2018). Comparative analysis between project delivery systems through quantitative assessment of project performance. Proc., Construction Research Congress 2018, American Society of Civil Engineers, New Orleans, Los Angeles, 670-681.

Ikpe, E., Hammon, F., and Oloke, D. (2012). Cost-benefit analysis for accident prevention in construction projects. Journal of Construction Engineering and Management, 138(8), 991-998. 
Karimi, Hossein, Timothy R. B. Taylor, Gabriel B. Dadi, Paul M. Goodrum, and Cidambi Srinivasan (2018). Impact of Skilled Labor Availability on Construction Project Cost Performance. Journal of Construction Engineering and Management, vol. 144(7), 1-10, doi:10.1061/(ASCE)CO.1943-7862.0001512.

Khanzadi, M., Nasirzadeh, F., and Dashti, M. S. (2018). Fuzzy cognitive map approach to analyze causes of change orders in construction projects. Journal of Construction Engineering and Management, 144(2), 04017111.

Love, P., M. Skitmore and G. Earl (1998) Selecting a suitable procurement method for a building project. Construction Management and Economics, 16:2, 221-233, doi:

10.1080/014461998372501

Love, P. E. D. (2002). Influence of project type and procurement method on rework costs in building construction projects. J. Constr. Eng. Manage., 128:1(18), 18-29, 10.1061/(ASCE) 07339364.

Love, P. E. D., and Edwards, D. (2004). Forensic project management: The underlying causes of rework in construction projects. Civ. Eng. Environ. Syst., 21(3), 207-228.

Love, P. E. D., P. Teo, and J. Morrison (2018). Revisiting quality failure costs in construction. J. Constr. Eng. Manage. 144 (2): 05017020. https://doi.org/10.1061/(ASCE)CO.1943 7862.0001427.

Luo, Lan, Qinghua He, Jianxun Xie, Delei Yang and Guangdong Wu (2017). Investigating the Relationship between Project Complexity and Success in Complex Construction Projects. Journal of Management in Engineering, 33 (2): doi:10.1061/(ASCE)ME.1943-5479.0000471.

MacQueen, J. (1967). Some Methods for Classification and Analysis of Multivariate Observations. Proceedings of the Fifth Berkeley Symposium on Mathematical Statistics and Probability, 1, 281-296.

Marle, F., and Vidal, L.-A. (2016). Managing Complex, High Risk Projects: A Guide to Basic and Advanced Project Management. Springer-Verlag, London, UK.

Mbachu, J., and Taylor, S. (2014). Contractual risks in the New Zealand construction industry: Analysis and mitigation measures. Int. J. Constr. Supply Chain Manage., 4(2), 22-33.

McTague, B., and G. Jergeas. (2002). Productivity Improvement on Alberta Major Construction Projects. Construction Productivity Improvement Rep. Edmonton, AB, Canada: Alberta Economic Development.

Menches, C. L., and Hanna, A. S. (2006a). Quantitative Measurement of Successful Performance from the Project Manager's Perspective. Journal of Construction Engineering and Management, 132(12), 1284-1293. 
Menches, C. L., and Hanna, A. S. (2006b). Conceptual Planning Process for Electrical Construction. Journal of Construction Engineering and Management, 132(12), 1306-1313. Merrow, E. W. (2011). Industrial Megaprojects: Concepts, Strategies, and Practices for Success. Hoboken, NJ: Wiley.

O’Connor, James T., and Brant D. Mock (2019). Construction, commissioning, and startup execution: problematic activities on capital projects. Journal of Construction Engineering and Management, 145(4), doi:10.1061/(ASCE)CO.1943-7862.0001621.

O’Connor, James T., Choi, Jin Ouk, and Matthew Winkler (2016). Critical success factors for mommissioning and start-up of capital projects.” Journal of Construction Engineering and Management, 142(11), 1-11, doi:10.1061/(ASCE)CO.1943-7862.0001179.

Olawale, Y., \& Sun, M. (2013). PCIM: Project control and inhibiting-factors management model. Journal of Management in Engineering, 29(1), 60-70.

Olupeliyawa, Asela M., Hughes, Chris, and Chinthaka D. Balasooriya (2009). A review of the literature on teamwork competencies in healthcare practice and training: Implications for undergraduate medical education. South East Asian Journal of Medical Education, 3 (2): 61-72,

Omar, M., and Fayek, A. Robinson (2014). A Framework for Identifying and Measuring Competencies and Performance Indicators for Construction Projects. In Proceedings of the Construction Research Congress, Atlanta, GA, May 19-21 2014. American Society of Civil Engineers, Reston, VA. pp. 2043-2052.

Orgut, R. E., M. Batouli, J. Zhu, A. Mostafavi, and E. J. Jaselskis (2020). Critical factors for improving reliability of project control metrics throughout project life cycle. Journal of Construction Engineering and Management, 36(1), 1-14, doi:10.1061/(ASCE)ME.19435479.0000710 .

Osei-Kyei, R., and Chan, A. P. C. (2017). Developing a project success index for public-private partnership projects in developing countries. Journal of Infrastructure Systems, 23(4), 04017028.

Parfitt, M. K., and Sanvido, V. E. (1993). Checklist of critical success factors for building projects. J. Manage. Eng., 9(3) 243-249, 10.1061/(ASCE)9742 -597X.

Peckiene, A., Komarovska, A., and Ustinovicius, L. (2013). Overview of risk allocation between construction parties. Procedia Eng., 57, 889-894.

Peña-Mora, F., C, Sosa,, and S. McCone, (2002), Dispute Avoidance and Resolution in Large Scale Civil Engineering Systems, MIT/Prentice Hall Civil and Environmental Engineering Textbook Series, USA.

Plain, C. (2007). Build an affinity for KJ method. Quality Progress, 40(3), 88.

PMI (2015). Capturing the Value of Project Management. 
Raheem, A. A., and Hinze, J. W. (2014). Disparity between construction safety standards: A global analysis. Safety Science, 70, 276-287.

Safapour, Elnaz, and Sharareh Kermanshachi (2019). Identifying Early Indicators of Manageable Rework Causes and Selecting Mitigating Best Practices for Construction. Journal of Management in Engineering, 35(2), doi:10.1061/(ASCE)ME.1943-5479.0000669.

Saunders, L. W., Kleiner, B. M., McCoy, A. P., Ellis, K. P., Smith-Jackson, T., and Wernz, C. (2017). Developing an inter-organizational safety climate instrument for the construction industry. Safety Science, 98(2017), 17-24.

Shrestha, K. K., and Shrestha, P. P. (2019). Change Orders on Road Maintenance Contracts: Causes and Preventive Measures. Journal of Legal Affairs and Dispute Resolution in Engineering and Construction, 11(3), 04519009.

Shrestha, P. P., and J. D. Fernane (2016). Performance of design-build and design-bid-build projects for public universities. Journal of Construction Engineering \& Management 143(3).

Sullivan, K. T. (2011). Quality management programs in the construction industry: Best value compared with other methodologies. J. Manage. Eng. 27 (4), 210-219, https://doiorg.ezproxy.library.wisc.edu/10.1061/(ASCE)ME.1943-5479.0000054.

Sweis, G., Sweis, R., Abu Hammad, A., and Shboul, A. (2008). Delays in construction projects: The case of Jordan. Int. J. Project Manage., 26(6), 665-674.

Taylor, T., and D. N. Ford (2006). Tipping point failure and robustness in single development projects. Syst. Dyn. Rev. 22 (1), 51-71, https://doi.org/10.1002/sdr.330.

Taylor, T. R. B., H. Karimi, P. M. Goodrum, and M. Albattah (2016). Is there a demographic craft labor cliff that will affect project performance? Construction Industry Institute Research Rep. 318-2. Austin, TX: Univ. of Texas-Austin.

Thomas, H. R., and M. J. Horman (2006). Fundamental principles of workforce management. J. Constr. Eng. Manage, 132 (1), 97-104. https://doi.org/10.1061/(ASCE)0733-

9364(2006)132:1(97).

U.S. Construction Industry - Statistics \& Facts (Statista 2018). Web link:

https://www.statista.com/topics/974/construction/

U.S Department of Labor (2017). Commonly Used Statistics. U.S. Department of Labor, https://www.osha.gov/oshstats/commonstats.html\&gt; (Accessed October 30, 2018).

Zaneldin, E. K. (2006). Construction claims in United Arab Emirates: Types, causes, and frequency. Int. J. Project Manage., 24(5): 453-459. 
Zou, P. X. W., Chen, Y., and Chan, T.-Y. (2010). Understanding and Improving Your Risk Management Capability: Assessment Model for Construction Organizations. Journal of Construction Engineering and Management, 136(8), 854-863. 